$$
\text { • }
$$


Printed at the Mathematical Centre, 49, 2 e Boerhaavestraat, Amsterdam.

The Mathematical Centre, founded the 11-th of February 1946, is a nonprofit institution aiming at the promotion of pure mathematics and its applications. It is sponsored by the Netherlands Government through the Netherlands Organization for the Advancement of Pure Research (Z.W.O), by the Municipality of Amsterdam, by the University of Amsterdam, by the Free University at Amsterdam, and by industries. 
MATHEMATICAL CENTRE TRACTS 28

J. OOSTERHOFF

\section{COMBINATION OF ONE-SIDED STATISTICAL TESTS}

SECOND PRINTING

MATHEMATISCH CENTRUM AMSTERDAM 1976 
AMS(MOS) subject classification scheme (1970): 62C07, 62C99

ISBN $906196041 \mathrm{x}$

first printing 1969

second printing 1976 


\section{ACKNOWLEDGEMENTS}

The present study is a continuation of previous work [60] by professor W.R. van Zwet and the author. The author has greatly benefited from challenging and inspiring discussions with professor van Zwet on many problems connected with the combination of tests.

The research on combination problems was performed at the Mathematisch Centrum. The author is much indebted to professor J. Hemelrijk, head of the Department of Mathematical Statistics, for his encouragement and stimulating criticism.

Dr.J. Fabius scrutinized the final draft of the manuscript and made many valuable comments resulting in considerable improvements of the text.

Ir. M.H. van Emden performed the computations which led to the construction of the exponential combination procedures of theorem 2.3.1 for the case $\mathrm{k}=3$.

The figures 2.5.1 through 2.5.9 were drawn by a plotter attached to the EL-X1 computer; most of the necessary ALGOL plotting programs were written by Mr. L.J.M. Geurts. 
. 
CONTENTS

Chapter

Page

1 INTRODUCTION

1.1 The combination problem

1.2 Historical survey 3

1.3 Preliminary decision theory 10

1.4 Convex and monotone acceptance regions 19

1.5 Totally positive functions 32

1.6 Most powerful tests against simple

alternatives

2 NORMALLY DISTRIBUTED STATISTICS WITH KNOWN VARIANCES

2.1 Large sample theory $\quad 44$

2.2 General properties $\quad 51$

2.3 The most stringent test 63

2.4 Asymptotically optimal procedures for $\alpha \rightarrow 0 \quad 76$

2.5 Numerical comparison of some combination procedures

3 COMBINATION OF ONE SAMPLE t-TESTS

3.1 Observations with equal variances 111

3.2 Observations with unequal variances 126

4 RELATED ASYMPTOTIC RESULTS

4.1 A general asymptotic approach 130

4.2 The likelihood ratio test in multinomial distributions 
CHAPTER 1. INTRODUCTION

1.1. THE COMBINATION PROBLEM

Suppose a number - say $\mathrm{k}$ - of experiments are performed to detect an effect. On many occasions the experiments are performed under different conditions and hence the magnitude of the effect may vary from experiment to experiment, if there is an effect at all. A standard example is the effect of a treatment on $\mathrm{k}$ different groups of patients. The question arises how to combine the information obtained from the different experiments.

In particular, if one wishes to test the hypothesis $H$ that there is no effect against the alternative $K_{1}$, that the effect is never negative and is positive in at least one of the experimental situations, it is common practice to test for a positive effect in each of the experimental situations separately and to combine the individual test statistics to obtain a test of $\mathrm{H}$ against $\mathrm{K}_{1}$. The problem is then how to combine the individual test statistics.

To be more precise, let the parameter $\theta_{i}$ describe the effect in the $i$-th experimental situation and let $\theta=\left(\theta_{1}, \ldots, \theta_{k}\right)$. The parameter space is the first orthant (boundaries included) of $\mathrm{k}$-dimensional Euclidean space $\mathbb{R}^{\mathrm{k}}$ and the testing problem may be formulated in the following way. For $i=1,2, \ldots, k$ let $\underline{t}_{i}$ denote a test statistic for testing $\theta_{i}=0$ against $\theta_{i}>0$ in the $i$-th experimental situation. The statistics $\underline{t}_{1}, \underline{t}_{2}, \ldots, t_{k}$ are supposed to be independent. On the basis of these statistics one wishes to test the hypothesis

$$
\mathrm{H}: \theta=0
$$

against the alternative

$$
K_{1}: \theta \cdot \geq 0
$$

where the symbol.$\geq$ indicates that the inequality is strict for at least one component; the symbol _. is similarly defined. We shall call this testing problem the one-sided combination problem, since under $K_{1}$ all the $\theta_{i}$ have the same sign. In the literature tests of $\mathrm{H}$ against the alternative

$$
\mathrm{K}_{2}: \theta \neq 0
$$


are usually described as combinations of two-sided tests, although it may be argued that testing $\mathrm{H}$ against $\mathrm{K}_{2}$ is not a combination problem at all, since in this case there is no relation between the parameters $\theta_{1}, \theta_{2}, \ldots, \theta_{k}$ : under $\mathrm{K}_{2}$ some of them may be positive and others negative. It would be appropriate to call the problem of testing $\mathrm{H}$ against the alternative

$$
\mathrm{K}_{3} \text { : either } \theta \cdot \geq \text { or } \theta \leq .0
$$

the two-sided combination problem, but to the author's knowledge it has so far received relatively little attention in the literature. It is essential to distinguish between these three testing problems, since the properties of combination procedures depend strongly on the alternatives considered.

Although quite a number of articles on the combination of tests have appeared, surprisingly little is known about the theoretical properties of combination procedures. Many authors confined their attention to the particular case where the true values of the parameters $\theta_{1}, \theta_{2}, \ldots, \theta_{k}$ are equal or to the still more restricted case where the statistics $t_{1}, t_{2}, \ldots, t_{k}$ are identically distributed both under $\mathrm{H}$ and the alternative hypothesis. In this last situation one often speaks of a goodness of fit problem (cf.[36] and [38]). Only a limited number of papers are concerned with the power of combination procedures. Without aiming at completeness we give a brief review of the literature on the combination of tests (in the wide sense) in section 1.2.

In the present study only the one-sided combination problem will be considered in some detail, since it is most frequently met in actual applications. The two-sided combination problem (testing $\mathrm{H}$ against $\mathrm{K}_{3}$ ) is more difficult; however, it will often be possible to deal with this problem by applying two one-sided combination procedures of size $\frac{1}{2} \alpha$ (where $\alpha$ is the desired significance level), because in most cases the overlap of the two critical regions is relatively small.

In section 3 of this chapter an outline of hypothesis testing is given. In section 1.4 tests with monotone and convex acceptance regions are considered in some detail. In section 1.5 the variation-diminishing property of integrals with totally positive kernels is mentioned; this property is crucial in some proofs of chapter 2. In section 1.6 most powerful tests against simple alternatives in exponential families are discussed. 
In chapter 2 the one-sided combination problem is first reduced to a standard problem of combining normally distributed random variables with unknown nonnegative expectations and unit variances by the asymptotic approach of J. NEYMAN and E.J.G. PITMAN. The most stringent combination procedure is then obtained for this standard problem in a few cases. It is also shown that the likelihood ratio combination procedure and FISHER's omnibus test are asymptotically optimal in some sense for this problem as the significance level tends to zero. In the last section of this chapter several combination procedures are compared numerically.

In chapter 3 the combination of STUDENT t-tests is considered. Restricting attention to invariant procedures, some results are obtained in the case where the observations have equal variances. However, it turns out that the more important case where the variances of the observations differ from sample to sample is essentially more difficult.

Finally in chapter 4 a general asymptotic approach to testing problems is discussed and a theorem on the asymptotic optimality of likelihood ratio tests in multinomial distributions is proved. The relevance of this approach to combination problems is also briefly considered.

\subsection{HISTORICAL SURVEY}

In early statistical work chi-square tests of goodness of fit were frequently applied. If the statistics $\underline{t}_{i}$ have (approximately) chi-square distributions under the hypothesis $H$, large values of the $\underline{t}_{i}$ indicating departures from $H$, a test of $H$ may be based on the sum of the $\underline{t}_{i}$. The additivity of random variables with chi-square distributions can then be used todetermine the appropriate critical value of the overall test. This is the simplest and presumably the oldest correct combination procedure. It is still often applied, e.g. when combining chi-square tests in $2 \times 2$ tables.

Most of the other combination procedures are based on the probability integral transformation. Let $p_{i}$ denote the one-sided tail probability of the statistic $\underline{t}_{i}(i=1,2, \ldots, k) ; p_{i}$ is the probability under $H$ of the event 
$t_{i} \geq t_{i}$ in case of upper-tailed tests and of the event $t_{i} \leq t_{i}$ in case of lower-tailed tests. If the $\underline{t}_{i}$ have continuous distribution functions under $H$, the $\underline{p}_{i}$ are uniformly distributed on the interval $[0,1]$ under $H$. Hence the hypothesis $\mathrm{H}$ may be tested by a chi-square test of goodness of fit applied to the $\underline{p}_{i}$ after a suitable division of the interval $[0,1]$ into subintervals. This combination method is mentioned in [38], but it is an approximate test that is applicable only if $\mathrm{k}$ is large and it is unsatisfactory even in that case.

In 1931 L.H.C. TIPPETT in the first edition of his book [51] described another test, attributed to him, that rejects $H$ for small values of the test statistic

(1.2.1) $\quad \min _{1 \leq i \leq k} \underline{p}_{i}$

In the continuous case the critical value of this test statistic is given by $1-(1-\alpha)^{1 / k}$, where $\alpha$ is the desired significance level of the test.

In the fourth edition of his famous book "Statistical methods for research workers" [17], which appeared in 1932, R.A. FISHER introduced his so-called omnibus procedure. This combination method rejects $H$ for small values of the statistic

$$
\prod_{i=1}^{k} \underline{p}_{i},
$$

i.e. for large values of

$$
\left.-2 \log \prod_{i=1}^{k} p_{i} \cdot *\right)
$$

If the $\underline{t}_{i}$ have continuous distribution functions, $-2 \log \mathrm{p}_{\mathrm{i}}$ has a chi-square distribution with 2 degrees of freedom under $\mathrm{H}$ and consequently (1.2.3) is distributed as chi-square with $2 \mathrm{k}$ degrees of freedom under $\mathrm{H}$.

In $1933 \mathrm{~K}$. PEARSON, independent of FISHER, wrote a long article [38] on the combination of tests based on the $p_{i}$. He was primarily interested in combining two-sided tests and was in doubt whether to reject $H$ for small values of the statistic (1.2.2) or for small values *) The symbol log will always denote the natural logarithm. 
of the statistic

$(1.2 .4) \quad \prod_{i=1}^{k}\left(1-p_{i}\right)$

(PEARSON defined $\underline{p}_{i}$ as the left-hand tail probability of $\underline{t}_{i}$ ). Under $H$ the distributions of $(1.2 .2)$ and $(1.2 .4)$ are of course identical. In the literature the test that rejects $H$ in the case of one-sided tests for large values of $(1.2 .4)$ is usually attributed to PEARSON, but this seems to be unjustified, since in all the examples of his paper $H$ is rejected for small values of the product test statistic. A year later F.N. DAVID [9] proposed to reject $H$ for small values of the minimum of both (1.2.2) and (1.2.4); a similar point of view was expressed in K. PEARSON's paper [39]. This proposal was of course motivated by the fact that they had two-sided individual tests in mind. In her introduction to the tables [10] DAVID advised the use of FISHER's procedure for the one-sided combination problem. For testing $\mathrm{H}$ against $\mathrm{K}_{2}$ she advised a test based on the two-sided tail probabilities that rejects $H$ for small values of the statistic

$$
\prod_{i=1}^{k} 2 \min \left\{\underline{p}_{i}, 1-p_{i}\right\}
$$

Under $\mathrm{H}$ this test statistic has, in the continuous case, the same distribution as $(1.2 .2)$ and $(1.2 .4)$. It appears that the criterion $(1.2 .5)$ has first been described by P.V. SUKHATME [50] in 1935.

Discussing the tests based on (1.2.2), (1.2.4) and (1.2.5) E.S. PEARSON [36] remarked in 1938 that a reasonable choice between different tests can only be made by specifying the possible alternatives and he derived, with the aid of the likelihood ratio principle, approximately optimal tests for some problems where the $\underline{t}_{i}$ are identically distributed.

A few years later W.A. WALLIS [54] gave a detailed exposition of FISHER's omnibus procedure and showed that the chi-square distribution of (1.2.3) under $\mathrm{H}$ is seriously invalidated when the $\underline{t}_{i}$ have discrete distributions. In the discrete case he suggested to compute exact tail probabilities of (1.2.2) by enumeration whenever possible. In 1949 H.O. LANCASTER [27] considered FISHER's test applied to chi-square test statistics $\underline{x}_{i}^{2}$ in sign tests and $2 \times 2$ tables, 
with two-sided alternatives, and compared its performance with the combination procedure based on the sum $\sum_{i} \underline{x}_{i}^{2}$ (without correction for continuity). He showed systematically by examples that the omnibus procedure loses much of its power in these testing problems, because the expectation under $\mathrm{H}$ of $-2 \log p_{i}$ is much smaller than 2 , the theoretical value in the continuous case. To combine discrete test statistics $\underline{t}_{i}$ he put forward two modifications of FISHER's procedure, called "mean $x^{2}$ " and "median $x^{2}$. He found that in case the true parameter values are equal, the two modifications of the omnibus procedure are slightly less powerful than the test based on the sum statistic in the examples considered. E.S. PEARSON [3T] suggested to deal with the difficulties in the discrete case by adding independent continuous random variables $\underline{v}_{i}$ to each $\underline{t}_{i}$ and applying FISHER's procedure to the sums $\underline{t}_{i}+\underline{v}_{i}$. If the sample spaces of the $\underline{t}_{i}$ are sets of consecutive integers (e.g. if the $\underline{t}_{i}$ are binomial, Poisson or hypergeometric random variables under $\mathrm{H}$ ), it is convenient to choose the $\underline{\mathrm{v}}_{\mathrm{i}}$ uniformly distributed on $[0,1]$; the tail probabilities $\underline{p}_{i}^{\prime}$ of $\underline{t}_{i}{ }^{+} \underline{v}_{i}$ are then easily determined with the aid of tables of random numbers. In this framework the "mean $x^{2}$ " and the "median $x^{2}$ " appear if $-2 \log \mathrm{p}_{i}^{\prime}$ is replaced by its conditional expectation or its conditional median respectively given $t_{i}(i=1,2, \ldots, k)$. As PEARSON recognized, the justification of introducing random elements $\mathrm{v}_{i}$ to obtain formally correct tests remains a matter of opinion.

For the combination of $2 \times 2$ tables (with binomial data) W.G. COCHRAN [7] advised pooling of all data in a single $2 \times 2$ table if homogeneity of the tables may be assumed, and if not, to add the square roots $\underline{x}_{i}$ of the individual chi-square test statistics $(i=1,2, \ldots, k)$, taking into account the signs of the differences between the estimated success probabilities. Under $\mathrm{H}$ this statistic has approximately a standard-normal distribution and tests can be based on it. In case the totals of the tables are widely different or the success probabilities to be compared have rather extreme values, COCHRAN advised another combination procedure that is based on the weighted sum of the estimated differences between the success probabilities to be compared. The weights are chosen in such a way that the test should be approximately optimal in the large sample case if the differences of the success probabilities involved are constant on the probit or logit scale. 
F. YATES expressed similar views in his articles [56] and [57] on the combination of $2 \times 2$ tables, stressing the importance of an analysis by maximum likelihood of the parameters involved. YATES also found in a number of examples that in the case of one-sided alternatives FISHER's test, applied to the $\underline{x}_{i}$, is not markedly influenced by the discreteness of the $\underline{x}_{i}$. He noted that FISHER's test must be expected to have considerably less power than the most powerful test of $H$ against alternatives of the form $\theta_{1}=\theta_{2}=\ldots=\theta_{k}>0$. Pooling of observations in a single $2 \times m$ table if several $2 \times m$ tables are to be combined has been suggested (without justification) by W.M. KINCAID [26].

In 1951 B. WILKINSON [55], generalizing TIPPETT's procedure, proposed to reject $H$ for small values of $\underline{p}_{(r)}$, the $r$-th smallest tail probability, and gave a table of critical values of this test statistic.

A. BIRNBAUM [4] was, in 1954, the first author to investigate the admissibility of certain combination procedures. For the one-sided combination problem, in the continuous case, he postulated that the tail probabilities $\underline{p}_{i}$, which have uniform distributions under $H$, have non-increasing densities under the alternative. This postulate is equivalent with monotone likelihood ratio of the distributions of the $\underline{t}_{i}$. A test based on the $\underline{p}_{i}$ is called monotone if rejection of $\mathrm{H}$ for a certain vector $\left(p_{1}, \ldots, p_{k}\right)$ implies rejection of $\mathrm{H}$ for any vector $\left(p_{1}^{*}, \ldots, p_{k}^{*}\right)$ such that $p_{i}^{*} \leq p_{i}$ for each $i$. BIRNBAUM showed that for any monotone test based on the $p_{i}$ one can find an alternative, represented by non-increasing densities of $\underline{p}_{1}, \underline{p}_{2}, \ldots, \underline{p}_{k}$, against which that test is most powerful. Moreover, if the distributions of the $\underline{t}_{i}$ are oneparameter exponential families, a combination procedure can only be admissible (i.e. cannot uniformly be improved upon) if its acceptance region in $\left(t_{1}, \ldots, t_{k}\right)$-space is convex. This condition also holds if twosided tests are combined. With the aid of this theorem (cf.[5]) BIRNBAUM showed that WILKINSON's procedure for $r>1$ and the procedure attributed to $K$. PEARSON are not admissible in the case of exponential family distributions. He recommended the use of FISHER's procedure or TIPPETT's test. Some years later T. LIPTAK [34], perhaps unaware of BIRNBAUM's work, proved that any combination procedure possessing some intuitively desirable properties (including a monotonicity property equivalent to that of BIRNBAUM) 
may be written in the form: reject $H$ for small values of the statistic

$$
\sum_{i=1}^{k} v_{i} \psi\left(p_{i}\right) \text {, }
$$

where $v_{i} \geq 0(i=1,2, \ldots, k)$ and $\psi$ is a strictly increasing function that is continuous on the interval $(0,1)$. In the particular case $\psi(p) \equiv \log p$ and equal weights $\nu_{i}$ FISHER's test appears. He again proved that any test generated by $(1.2 .6)$ is most powerful against some simple alternative and showed that the procedure based on (1.2.6) is unbiased if each of the tests to be combined is unbiased. LIPTAK suggests as a suitable choice of the function $\psi$ the inverse $\Phi^{-1}$ of the standard-normal distribution function $\Phi$; with this choice the distribution of the statistic (1.2.6) is normal under $\mathrm{H}$ in the continuous case.

If in $(1,2.6)$ we choose $\psi(p) \equiv \log p$, with arbitrary weights $\nu_{i}$, a weighted version of FISHER's test is obtained that rejects $H$ for small values of the statistic

$$
\prod_{i=1}^{k} p_{i}{ }^{\nu}
$$

This test was first considered by I.J. GOOD [18], who derived the distribution of the statistic (1.2.7) under $\mathrm{H}$ in the case that all $v_{i}$ are different. In a further paper [19] GOOD gave a highly subjective discussion on the choice of the weights $v_{i}$ in this test. M. ZELEN [58] noted that the problem of combining two variance-ratios (F-tests) often arises in the analysis of incomplete block designs and proposed a weighted FISHER procedure for such a problem. In a joint paper [59] with L.S. JOEL the combination of two variance-ratios (with equal numbers of degrees of freedom of the numerators) by means of the procedure (1.2.7) is thoroughly discussed; they proposed a choice of the weights and gave tables of the power of some weighted FISHER procedures for this problem.

In $1961 \mathrm{H} .0$. LANCASTER [28] considered three transformations of the tail probabilities $\mathrm{p}_{\mathrm{i}}$ : either to $-2 \log \mathrm{p}_{\mathrm{i}}$, or to a normal random variable $\underline{x}_{i}=\Phi^{-1}\left(\underline{p}_{i}\right)$ - LIPTAK's proposal -, or to a chi-square random variable $\underline{x}_{S}^{2}$. with $s_{i}$ degrees of freedom. The overall test statistic is then obtained by addition of the respective random variables. In the last two cases weights 
can be introduced as multipliers of the $\underline{x}_{i}$ or by varying the degrees of freedom $s_{i}$ of $\underline{x}_{s}^{2}$; the distributions under $H$ remain very simple and the complicated distribution of the weighted FISHER procedure is avoided. LANCASTER came to the conclusion that in the case of equal weighting there is no great difference between the three combination methods. He also compared the power of the sign test applied to a sample of 20 observations in the case of one-sided alternatives with the power of the "mean $x^{2}$ " procedure when applied to 2,3 or 4 subsamples, assuming equal probabilities of success. He found that the power of the "mean $x^{2}$ " test is not much smaller than that of the (most powerful) sign test.

Notwithstanding these various developments, many statisticians prefer to use linear combinations $\sum_{i} v_{i}$ of the individual test statistics to test the hypothesis $H$. Since many test statistics are approximately normally distributed in the large sample case, the distribution of $\sum v_{i} \underline{t}_{i}$ can be approximated fairly well by a normal distribution in the null-case in most applications. The choice of the weights $\nu_{i}$ is then the essential problem.

PH. VAN ELTEREN [16] discussed linear combination of two-sample tests of WILCOXON. He considered two choices of the weights involved. The first set of weights has the property that the alternatives, against which the combination procedure is consistent, do not depend on the ratios of the individual sample sizes. Such tests are called designfree; for a discussion of designfree tests we refer to C. VAN EEDEN and J. HEMELRIJK [13]. The second set of weights was introduced to obtain a locally best combination procedure (for "equal" alternatives). The robustness of linear combinations of two-sample tests of WILCOXON if there are small variations in the variances of the observations is investigated by M.L. PURI in a forthcoming paper [40].

W. SCHAAFSMA ([45], [46]) considered linear combination of test statistics when the test statistics are normally distributed and the alternative is one-sided. He proposed the use of most stringent somewhere most powerful tests (cf. also [44]) and constructed such tests for various testing problems. It turns out that for the one-sided combination problem such tests are essentially linear combinations of the individual test statistics with appropriate weights. In his opinion these tests cannot be improved upon to 
a "worthwhile" extent. In [46] he compared these tests with the (unrestricted) most stringent combination procedures, which were first derived in [59].

A sampling experiment has been performed by N. BHATTACHARYA [2], who considered the combination of chi-square statistics, which have central distributions under $\mathrm{H}$ and non-central distributions under the alternative. Varying the degrees of freedom and the non-centrality parameters, he compared three procedures: FISHER's test, the sum test and TIPPETT's test. He found that the first two tests are about equally powerful in all cases considered, but that the power of TIPPETT's test is much smaller, except possibly in the case where only one of the variables has a non-central distribution.

Recently R.B. DAVIES ([11], [12]) approached the combination problem in a somewhat different manner, as he supposed that the parameters $\theta_{i}$ measuring the effect are random variables with normal $N\left(0, \sigma^{2}\right)$ distributions and the hypothesis tested is $\sigma^{2}=0$ against $\sigma^{2}>0$. For this combination problem he constructed $\beta$-optimal size- $\alpha$ tests (see section 1.3). Some power comparisons with FISHER's procedure (with of without weighting) are made in [12], partly based on simulation.

A study of the decision theoretical aspects of the one-sided combination problem was made by W.R. VAN ZWET and J. OOSTERHOFF [60]. The present work is a continuation of this research and extends and generalizes the results of $[60]$.

\subsection{PRELIMINARY DECISION THEORY}

In this sections we recall some concepts of the theory of testing hypotheses and the related decision theory and introduce some notation.

We shall be concerned with a measurable space $(X, \hat{z})$, where $X$ is Euclidean and $\mathcal{A}_{\text {is }}$ the $\sigma$-algebra of Borel sets of $\mathrm{X}$. Let $\Omega$ be a Borel subset of $r$-dimensional Euclidean space $\mathbb{R}^{r}$ and

$$
\rho_{\Omega}=\left\{P_{\theta} \mid \theta \in \Omega\right\}
$$

a family of probability measures defined over $(X, \Omega)$ and dominated by a $\sigma$-finite measure $\lambda$. In applications $\lambda$ will usually be either Lebesque measure 
or counting measure. The measures $P_{\theta}$ are supposed to be different, i.e. for every pair $\theta^{\prime}, \theta^{\prime}, \epsilon \Omega$ a set $A \in \Omega$ exists for which $P_{\theta},(A) \neq P_{\theta},{ }^{\prime}(A)$. According to the RADON-NIKODYM theorem there exists for any $\theta \in \Omega$ a probability density $p(x ; \theta)$ with respect to $\lambda$ such that

$$
P_{\theta}(A)=\int_{A} p(x ; \theta) d \lambda(x) \text { for all } A \in \mathcal{A} \text {. }
$$

The index set $\Omega$ will be called the parameter space.

Let $\underline{x}$ denote an observable random vector assuming values in $X$ and inducing one of the measures $P_{\theta}$ in $(x, \hat{q})$, i.e.

$$
\operatorname{Pr}(\underline{x} \in A)=P_{\theta}(A) \quad \text { for all } A \in \Omega \text {. }
$$

Without confusion we shall often write $P_{\theta}(\underline{x} \in A)$.

Let $\Omega_{\mathrm{H}}$ and $\Omega_{\mathrm{K}}$ denote two disjoint subsets of $\Omega$. Suppose we wish to test the hypothesis

$$
\mathrm{H}: \theta \in \Omega_{\mathrm{H}}
$$

against the alternative

$$
\mathrm{K}: \theta \in \Omega_{\mathrm{K}} \text {. }
$$

A test of $H$ against $K$ is characterized by a critical function $\delta(x)$, the probability that $\mathrm{H}$ is rejected in favor of $\mathrm{K}$ for a given outcome $\underline{x}=\mathrm{x}$ in the sample space $X$. Such a critical function $\delta$, a measurable mapping of $\mathrm{X}$ into the closed interval $[0,1]$, is said to be nonrandomized if $\delta(x)=0$ or 1 for all $x \in X$. A test will often be indicated by its critical function $\delta$. If $\delta$ is nonrandomized, the set $\{x \mid \delta(x)=0\}$ is called the acceptance region and the set $\{x \mid \delta(x)=1\}$ the critical region of the test $\delta$. It is convenient to introduce a convergence definition in the space of all critical functions. A sequence $\left\{\delta_{n}\right\}$ of critical functions is said to be weakly convergent to a weak limit $\delta$ if

$$
\text { (1.3.1) } \quad \lim _{n \rightarrow \infty} \int \delta_{n}(x) f(x) d \lambda(x)=\int \delta(x) f(x) d \lambda(x)
$$

for all $\lambda$-summable $f$. We note that a sequence $\left\{\delta_{n}\right\}$ is weakly convergent 
to $\delta$ if and only if

$$
\lim _{n \rightarrow \infty} \int_{A} \delta_{n}(x) d \lambda(x)=\int_{A} \delta(x) d \lambda(x)
$$

for all $A \in \mathcal{A}$ with $\lambda(A)<\infty$.

This convergence definition coincides with the concept of regular convergence introduced by A. WALD [53]. The celebrated weak compactness theorem (cf.

E.L. LEHMANN [31] p. 354) asserts that the space of all critical functions is compact in the topology of weak convergence.

We shall be concerned with tests at a fixed significance level $\alpha(0<\alpha<1)$, that is, with critical functions $\delta$ whose size

$$
\left.\sup _{\theta \in \Omega_{\mathrm{H}}} \mathrm{E}_{\theta} \delta(\underline{\mathrm{x}}) \quad *\right)
$$

does not exceed $\alpha$. The class of all such tests will be denoted by $D(\alpha)$. The power function of a test $\delta$ will be denoted by

$$
\beta_{\delta}(\theta) \stackrel{\operatorname{def}}{=} E_{\theta} \delta(\underline{x})
$$

If we wish to test a simple hypothesis $\theta=\theta^{0}$ against a simple alternative $\theta=\theta^{1}$, the most powerful level- $\alpha$ test is given by the fundamental NEYMANPEARSON lemma:

$$
\delta(x)=\left\{\begin{array}{lll}
1 \text { if } & p\left(x ; \theta^{1}\right)>c_{\alpha} p\left(x ; \theta^{0}\right) \\
\gamma_{\alpha} \text { if } & p\left(x ; \theta^{1}\right)=c_{\alpha} p\left(x ; \theta^{0}\right) \\
0 \text { if } & p\left(x ; \theta^{1}\right)<c_{\alpha} p\left(x ; \theta^{0}\right),
\end{array}\right.
$$

where $c_{\alpha}$ and $\gamma_{\alpha}\left(0 \leq \gamma_{\alpha} \leq 1\right)$ must be determined to satisfy

$$
{ }_{\theta}^{E} \delta(\underline{x})=\alpha \text {. }
$$

If the alternative is composite, i.e. if $\Omega \mathrm{K}$ contains more than one point, a uniformly most powerful (UMP) level- $\alpha$ test (maximizing $\beta_{\delta}(\theta)$ for all $\theta \in \Omega_{K}$

${ }^{*)} \mathrm{E}_{\theta}$ denotes the expectation under $\mathrm{P}_{\theta}$. 
simultaneously among level- $\alpha$ tests $\delta$ of $\mathrm{H}$ ) usually does not exist. In such cases it is common practice to restict attention to a subclass $\bigcap^{*}(\alpha) \subset \supset(\alpha)$ of tests possessing some desirable property and to select the UMP test within $g^{*}(\alpha)$, i.e. a level- $\alpha$ test $\delta_{0}$ satisfying

$$
\beta_{\delta_{0}}(\theta) \geq \beta_{\delta}(\theta) \quad \text { for all } \delta \in D^{*}(\alpha) \text { and } \theta \in \Omega_{K} \text {, }
$$

provided such a test exists. Well-known choices for $D^{*}(\alpha)$ are

(i) the class of unbiased level- $\alpha$ tests, satisfying

$$
\beta_{\delta}(\theta) \geq \alpha \quad \text { for all } \theta \in \Omega_{\mathrm{K}} \text {, }
$$

(ii) the class of similar size- $\alpha$ tests, satisfying

$$
\beta_{\delta}(\theta)=\alpha \quad \text { for all } \theta \in \Omega_{\mathrm{H}} \text {, }
$$

(iii) the class of invariant level- $\alpha$ tests. In order to define this last class consider a group $G$ of measurable 1-1 transformations $g$ of the sample space $X$. Let to any $g \in G$ correspond a transformation $\bar{g}$ of the parameter space $\Omega$ such that

$$
P_{\theta}(A)=P_{\bar{g} \theta}(g A) \text { for all } A \in \mathcal{A} \text {. }
$$

The transformations $\bar{g}$ induced by the elements $g$ of $G$ also constitute a group $\bar{G}$ acting on $\Omega$. We say that the problem of testing $H$ against $K$ remains invariant under $G$ if

$$
\overline{\mathrm{g}} \Omega_{\mathrm{H}}=\Omega_{\mathrm{H}} \text { and } \overline{\mathrm{g}} \Omega_{\mathrm{K}}=\Omega_{\mathrm{K}} \quad \text { for all } \overline{\mathrm{g}} \in \overline{\mathrm{G}} \text {. }
$$

A test $\delta$ is then called invariant under $G$ if

$$
\delta(g x)=\delta(x) \quad \text { for all } x \in X \text { and } g \in G \text {. }
$$

A statistic $T(\underline{x})$ is said to be a maximal invariant under $G$ if it is invariant and if $\mathrm{T}\left(\mathrm{x}_{1}\right)=\mathrm{T}\left(\mathrm{x}_{2}\right)$ implies $\mathrm{x}_{2}=\mathrm{gx}$, for some $\mathrm{g} \in \mathrm{G}$. A maximal invariant $\tau(\theta)$ under $\bar{G}$ is similarly defined. A test $\delta$ is invariant under $G$ if and only 
if there exists a measurable function $h$ such that

$$
\delta(\mathrm{x})=\mathrm{h}[\mathrm{T}(\mathrm{x})] \quad \text { for all } \mathrm{x} \in \mathrm{X} \text {, }
$$

where $T$ is a maximal invariant. The distribution of $T(\underline{x})$ only depends on $\tau(\theta)$ and the same property holds a fortiori for an invariant critical function $\delta(\underline{x})$.

Unfortunately the combination problems to be discussed do not admit UMP tests among one of the classes (i), (ii) or (iii). In such cases one may decide to select a minimax test. There exist several types of minimax tests. Our main interest will be focussed on most stringent (MS) tests. Let

$$
\beta_{\alpha}^{+}(\theta) \quad \text { def } \sup _{\delta \in \Phi(\alpha)} \beta_{\delta}(\theta)
$$

denote the level- $\alpha$ envelope power function and consider a particular level- $\alpha$ test $\delta$. The shortcoming of this test (with respect to envelope power) is the amount by which the power of the test $\delta$ falls short of the envelope power, i.e.

$$
\text { (1.3.3) } \quad R_{\delta}(\theta) \stackrel{\text { def }}{=} \beta_{\alpha}^{+}(\theta)-\beta_{\delta}(\theta) \text {. }
$$

A level- $\alpha$ test $\delta_{0}$ is called MS if

$$
\text { (1.3.4) } \sup _{\theta \in \Omega_{\mathrm{K}}} \mathrm{R}_{\delta_{0}}(\theta)=\inf _{\delta \in \Phi(\alpha)} \sup _{\theta \in \Omega_{\mathrm{K}}} \mathrm{R}_{\delta}(\theta) \text {, }
$$

i.e. if $\delta_{0}$ minimizes the maximum shortcoming over $\Omega_{\mathrm{K}}$ among level- $\alpha$ tests. The shortcoming $R_{\delta}(\theta)$ of a test $\delta$ may be interpreted as expected loss (and hence as a risk function in the usual sense of decision theory) if we define the loss functions

$$
L_{1}(\theta)=\beta_{\alpha}^{+}(\theta)-1 \quad \text { when rejecting } H
$$

and

$$
L_{2}(\theta)=\beta_{\alpha}^{+}(\theta) \quad \text { when accepting } H \text {. }
$$


Another approach towards a minimax solution is as follows. Let $d$ be a nonnegative function defined over $\Omega$ such that $d(\theta)=0$ for all $\theta \epsilon \Omega_{H} ; d(\theta)$ may be interpreted as a distance of $\theta \epsilon \Omega$ to $\Omega_{\mathrm{H}}$. Let $\Omega_{\mathrm{K}}^{\prime}$ be defined by

$$
\Omega_{\mathrm{K}}^{\prime}=\left\{\theta \mid \mathrm{d}(\theta) \geq \Delta, \theta \in \Omega_{\mathrm{K}}\right\}
$$

for some positive $\Delta$. Typically $\Omega_{K}^{\prime}$ is the set of parameter values differing so widely from those postulated by the hypothesis $H$ that false acceptance of $H$ is a serious error whenever $\theta \epsilon \Omega_{K}^{\prime}$. A level- $\alpha$ test $\delta_{0}$ is called a maximin test of $\mathrm{H}$ with respect to $\Omega_{\mathrm{K}}^{\prime}$ if

$$
\inf _{\theta \in \Omega_{K}^{\prime}} \beta_{\delta_{0}}(\theta)=\sup _{\delta \in \Phi(\alpha)} \inf _{\theta \in \Omega_{K}^{\prime}} \beta_{\delta}(\theta),
$$

i.e. if the test $\delta_{0}$ maximizes the minimum power over $\Omega_{K}^{\prime}$ among level- $\alpha$ tests. A maximin test can also be interpreted as a minimax procedure since it minimizes the maximum of the simple risk function $1-\beta_{\delta}(\theta)$ over $\Omega_{K}^{\prime} \cdot A$ serious drawback of maximin tests is their dependence on the often rather arbitrary choice of $\Delta$ ( in a few cases there is no dependence on $\Delta$, but this seems to be exceptional).

A related class of procedures, $\beta$-optimal tests, have been introduced by R.B. DAVIES [11] for the combination problem. For a given $\beta(\alpha<\beta \leq 1)$ a level- $\alpha$ test $\delta_{0}$ is called $\beta$-optimal with respect to the distance function $d$ if $\delta_{0}$ minimizes the expression

$$
\inf \left\{\Delta \mid \beta_{\delta}(\theta) \geq \beta \text { for all } \theta \text { satisfying } a(\theta)>\Delta\right\}
$$

among tests $\delta \in D(\alpha)$. One might say that a $\beta$-optinal test minimizes (in a sense) the zone of indifference for a predetermined minimum power. Obviously the $\beta$-optimal tests depend in general on the choice of $\beta$.

We remark that both MS tests and maximin tests always exist in the present context, as is easily verified by application of the weak compactness theorem. If the testing problem is invariant under a transformation group G, a MS test which is invariant under $G$ exists under rather general conditions. This is formulated in the following lemma. 
Lemma 1.3 .1

Let $G$ be a group of tranformations of $X$ such that the induced group $\bar{G}$ leaves $\Omega_{\mathrm{H}}$ and $\Omega_{\mathrm{K}}$ invariant. Let $B$ be a $\sigma$-algebra of subsets of $G$ such that for any $A \in \mathcal{A}$ the set of pairs $(x, g)$ with $g x \in A$ is an element of $\mathcal{A}_{x} B$ and for any $B \in B$ and $g \in G$ the set $B g$ is $B$ - measurable. Let $G$ be either finite, or, if $G$ is infinite, let there exist a sequence of probability measures $\nu_{n}$ over $(G, B)$ and a $\sigma$-finite measure $\nu$ over $(G, \beta)$ such that

$$
\lim _{n \rightarrow \infty}\left|\nu_{n}(B g)-\nu_{n}(B)\right|=0 \text { for all } g \in G \text { and } B \in B
$$

and

$$
v(B)=0 \text { implies } v(\mathrm{Bg})=0 \text { for all } g \in \mathrm{G} \text {. }
$$

Then there exists, for any $\alpha$, a MS level- $\alpha$ test of $H$ against $K$ that is invariant.

The proof of this well-known result is standard. It is based on the HUNT-STEIN theorem and the fact that the set of parameter points for which $\beta_{\alpha}^{+}(\theta)$ is constant is invariant under $\bar{G}$. The condition (1.3.6) of the lemma ensures that the MS test is actually invariant and not merely almost invariant (cf. LEHMANN [31] th.4 of ch.6). Finally we remark that under the same conditions invariant maximin tests also exist.

The minimax principle sometimes leads to quite unreasonable tests in the sense that, although they minimize the maximum risk, the power of such tests may be relatively small for "most" alternatives. Moreover, minimax tests are often quite difficult to find and may have a very complicated character. These considerations led W. SCHAAFSMA ( [44], [45], [46]) to the introduction of most stringent somewhere most powerful (MSSMP) tests. A level- $\alpha$ test is called MSSMP if it is MS among the class of level- $\alpha$ tests which are most powerful against simple alternatives in $\Omega_{\mathrm{K}}$.

Let $\mathcal{K}$ denote a $\sigma$-algebra of subsets of $\Omega_{K}$. We assume that $p(x ; \theta)$, considered as a function of $\mathrm{x}$ and $\theta$, is measurable $\hat{A} \times \mathcal{K}$. A probability distribution $\xi$ over $\left(\Omega_{K}, \mathcal{K}\right)$ is called a prior distribution. A level- $\alpha$ test $\delta$ of $\mathrm{H}$ is said to be Bayes with respect to a prior distribution $\xi$ if it maximizes the expression 


$$
\int_{\Omega_{K} \beta^{\prime}}(\theta) d \xi(\theta)
$$

among all $\delta^{\prime} \in 9(\alpha)$. Equivalently, by FUBINI's theorem, $\delta$ is Bayes with respect to $\xi$ if $\delta$ is a most powerful test of $\mathrm{H}$ against the simple alternative that $\underline{x}$ is distributed according to the probability density

$$
\mathrm{h}(\mathrm{x} ; \xi)=\int_{\Omega_{\mathrm{K}}} \mathrm{p}(\mathrm{x} ; \theta) \mathrm{d} \xi(\theta)
$$

with respect to $\lambda$. The class of all level- $\alpha$ Bayes tests of $H$ against $K$ will be denoted by $\beta(\alpha)$. The power of a test $\delta$ against an alternative of the form (1.3.7) will be denoted by

$$
\beta_{\delta}(\xi)=\int_{X} \delta(x) h(x ; \xi) d \lambda(x)=\int_{\Omega_{K}} \beta_{\delta}(\theta) d \xi(\theta)
$$

Extending the definition of the shortcoming of a level- $\alpha$ test $\delta$ to arguments $\xi$,

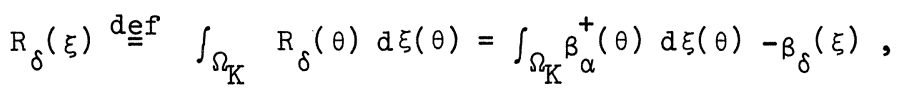

it is obvious that a level- $\alpha$ test $\delta_{0}$ is Bayes with respect to a prior distribution $\xi_{0}$ if it minimizes $R_{\delta}\left(\xi_{0}\right)$ among all $\delta \in \rho(\alpha)$.

The level- $\alpha$ minimax shortcoming $\mathrm{R}_{\alpha}^{+}$is defined as

$$
\mathrm{R}_{\alpha}^{+} \text {def } \inf _{\delta \in \mathfrak{D}(\alpha)} \sup _{\theta \in \Omega_{\mathrm{K}}} \mathrm{R}_{\delta}(\theta) ;
$$

it is equal to the maximum shortcoming of a MS level- $\alpha$ test (cf.(1.3.4)). If the level- $\alpha$ minimax shortcoming is sufficiently small, the MS procedure (or any level- $\alpha$ test with only slightly larger maximum shortcoming) may be a satisfactory solution of the testing problem.

A prior distribution $\xi_{0}$ over $\left(\Omega_{K}, K\right)$ is said to be least favorable (LF) at level $\alpha$ if

$$
\inf _{\delta \in D(\alpha)} R_{\delta}\left(\xi_{0}\right) \geq \inf _{\delta \in D(\alpha)} R_{\delta}(\xi) \text { for all } \xi ;
$$

it is the prior distribution that is hardest to distinguish from the null hypothesis at level $\alpha$ in the sense of the risk function $R$. We note that the LF character of a prior distribution heavily depends on the risk function; 
changing $R$ will in general entail that another prior distribution becomes LF.

Any MS test is the weak limit of a sequence of Bayes tests (WALD [53] th.3.12). If a LF prior distribution $\xi_{0}$ exists, any MS level- $\alpha$ test is Bayes with respect to $\xi_{0}$ (WAID [53]th.3.9). A prior distribution $\xi_{0}$ and a corresponding level- $\alpha$ Bayes test $\delta_{0}$ constitute a LF prior distribution and a MS procedure respectively if and only if $\xi_{0}$ assigns probability one to the set of parameter points for which $R_{\delta_{0}}(\theta)$ assumes its absolute maximum (WALD [53] th.3.10, 3.11).

However, a LF prior distribution does not necessarily exist. WALD ([52]th.5.9) has shown that LF prior distributions exist under the assumption that $\mathrm{p}(\mathrm{x} ; \theta)$ is continuous in $\theta$ and $\Omega_{\mathrm{K}}$ is compact. The compactness assumption is rather restrictive and unnecessarily strong. LEHMANN [30] has shown that the compactness condition can be replaced by a weaker condition, but the risk functions considered by him do not include the shortcoming ( he only considered risk functions of the form $L(\theta) \mathrm{E}_{\theta}(1-\delta(\underline{\mathrm{x}})$ ) where $L(\theta)$ is some loss function).

A level- $\alpha$ test $\delta$ is said to be dominated by a level- $\alpha$ test $\delta^{\prime}$ if

$$
\beta_{\delta}(\theta) \leq \beta_{\delta^{\prime}}(\theta) \quad \text { for all } \theta \in \Omega_{K}
$$

with strict inequality at least once, or equivalently, if

$$
R_{\delta}(\theta) \geq R_{\delta^{\prime}}(\theta) \quad \text { for all } \theta \in \Omega_{K}
$$

with strict inequality at least once. A level- $\alpha$ test $\delta$ is called admissible if no level- $\alpha$ test $\delta^{\prime}$ dominating $\delta$ exists; otherwise $\delta$ is said to be inadmissible. A class $C$ of level- $\alpha$ tests is said to be complete if for any level- $\alpha$ test not in $C$ there exists a $\delta^{\prime}$ in $C$ dominating it. A minimal complete class is a complete class not containing a complete proper subclass. A class $C$ of level- $\alpha$ tests is said to be essentially complete if for any level-a test $\delta$ there exists a test $\delta^{\prime}$ in $C$ such that $(1.3 .11)$ is satisfied (possibly with equality for all $\theta \in \Omega_{\mathrm{K}}$ ).

If Bayes tests are unique a.e. $[\lambda]$, then any Bayes test is admissible. But in general there are inadmissible Bayes tests and admissible tests which 
are not Bayes. The importance of Bayes tests is nevertheless emphasized by the following complete class theorem (cf. WALD [53] th.3.18 and L. LECAM [29] th.4), which we state in the form of a lemma.

Lemma 1.3 .2

For testing the hypothesis $\mathrm{H}$ against $\mathrm{K}$ at level $\alpha$ the closure (in the topology of weak convergence) of the class $B(\alpha)$ of level- $\alpha$ Bayes tests constitutes an essentially complete class.

The closure of $\beta(\alpha)$ is complete if tests with identical risk functions on $\Omega_{\mathrm{K}}$ are equal a.e. $[\lambda]$. This property is certainly satisfied if the family of probability measures $\rho_{\Omega_{\mathrm{K}}}$ is boundedly complete, i.e. if

$$
\int_{X} f(x) p(x ; \theta) d \lambda(x)=0 \text { for all } \theta \in \Omega_{K}
$$

implies $f(x)=0$ a.e. $[\lambda]$ for any bounded measurable function $f$. If $\rho \Omega_{K}$ is an exponential family and $\Omega_{\mathrm{K}}$ contains a $\mathrm{r}$-dimensional rectangle, $\rho_{\Omega_{\mathrm{K}}}$ is boundedly complete and hence the closure of $\beta(\alpha)$ is a complete class in this case.

$B(\alpha)$ is itself a complete class if $\Omega_{K}$ is compact and $p(x ; \theta)$ is continuous in $\theta$. In this case weak limits of Bayes tests are also Bayes (WALD [53]th.5.5).

\subsection{CONVEX AND MONOTONE ACCEPTANCE REGIONS}

In the last twenty years an extensive literature on admissibility of statistical tests and complete classes of tests has appeared. Especially tests of hypotheses in exponential families of distributions have been thoroughly studied. It is well known that in this case nonrandomized tests with convex acceptance regions are of fundamental importance. In the first part of this section we mention some theorems on this subject and prove a related result. If the hypotheses are one-sided, the monotonicity of the acceptance regions plays an important part. This is shown in the second part of this section.

We again consider the family $\rho_{\Omega}$ of probability measures dominated 
by a $\sigma$-finite measure $\lambda$ over $(x, \mathcal{A})$, introduced in section 1.3. Suppose we wish to test the hypothesis

$$
\mathrm{H}: \theta \in \Omega_{\mathrm{H}}
$$

against the alternative

$$
\mathrm{K}: \theta \in \Omega_{\mathrm{K}},
$$

where $\Omega_{\mathrm{H}}$ and $\Omega_{\mathrm{K}}$ are disjoint subsets of $\Omega$. First we shall assume that the null hypothesis is simple:

$$
\Omega_{\mathrm{H}}=\left\{\theta^{0}\right\} \text {. }
$$

Let $e_{0}(\alpha)$ denote the set of all size- $\alpha$ critical functions for testing $\theta=\theta^{0}$ which are equal a.e. $[\lambda]$ to any critical function $\delta$ satisfying

$$
\delta(x)=\left\{\begin{array}{l}
0 \text { if } x \in \operatorname{int}(A) \\
1 \text { if } x \in X-A,
\end{array}\right.
$$

where $A$ is some closed convex set in $\mathbb{R}^{k}$ and $\operatorname{int}(A)$ denotes the interior of $A$. We shall say that $C_{0}(\alpha)$ is the set of size- $\alpha$ tests of $\theta=\theta^{0}$ with a.e. convex acceptance regions. To prove that $e_{0}(\alpha)$ is an (essentially) complete class of tests for testing $\theta=\theta_{0}$ against $\theta \in \Omega_{K}$, a possible line of attack is to show first that the class $B_{0}(\alpha)$ of level- $\alpha$ Bayes tests of $\theta=\theta^{0}$ is a subset of $e_{0}(\alpha)$. Since the closure of $\beta_{0}(\alpha)$ is an essentially complete class (lemma 1.3.2), the essential completeness of $e_{0}(\alpha)$ is then established if $e_{0}(\alpha)$ is closed in the topology of weak convergence. This last property, stated with an incorrect proof by A. BIRNBAUM [5] in a special case, has been proved in full generality by T.K. MATTHES and D.R. TRUAX ([35]th.2.1) with the aid of the BLASCHKE selection theorem.

Lemma 1.4 .1 (T.K. MATTHES and D.R. TRUAX)

For any $\sigma$-finite measure $\lambda$ dominating the family $\rho_{\Omega}$ the class $e_{0}(\alpha)$ is closed in the topology of weak convergence. 
The line of argument sketched above has proved to be very useful if $\rho_{\Omega}$ is an exponential family, i.e.

$$
p(x ; \theta)=C(\theta) \exp \left[\sum_{i=1}^{k} \theta_{i} x_{i}\right], \theta \in \Omega,
$$

with respect to an appropriate measure $\lambda$, where $\theta=\left(\theta_{1}, \ldots, \theta_{k}\right)$ and $\mathrm{x}=\left(\mathrm{x}_{1}, \ldots, \mathrm{x}_{\mathrm{k}}\right)$ are $\mathrm{k}$-dimensional vectors $(\mathrm{k} \geq 2)$ and $\Omega$ is the (convex) natural parameter space, i.e. the set of all $\theta$ satisfying $\int \exp \left[\left[\theta_{i} x_{i}\right] d \lambda(x)<\infty\right.$. The family $(1.4 .2)$ is not the most general exponential family, which is usually defined as

$$
p(x ; \theta)=C(\theta) \exp \left[\sum_{i=1}^{k} Q_{i}(\theta) T_{i}(x)\right], \theta \in \Omega,
$$

but this form reduces to $(1 \cdot 4.2)$ after suitable transformations.

The following well-known result has first been obtained by BIRNBAUM [5] in a particular case (cf. also [35]).

Lemma 1.4.2 (A. BIRNBAUM)

Let $\underline{x}=\left(\underline{x}_{1}, \ldots, \underline{x}_{k}\right)$ be distributed according to the density (1.4.2) with respect to the measure $\lambda$. Then $e_{0}(\alpha)$ is an essentially complete class for testing $\theta=\theta^{0}$ against $\theta \in \Omega_{K}$. If $\Omega_{K}$ contains a $k$-dimensional rectangle and $P_{\theta}$ and $\lambda$ are equivalent, $e_{0}(\alpha)$ is complete.

BIRNBAUM then investigates the absolutely continuous case more closely and shows that the closure of $B_{0}(\alpha)$ coincides with $e_{0}(\alpha)$ under the additional assumption that $\Omega$ contains hyperspheres of arbitrarily large radii. Here it is assumed that $\Omega_{K}=\Omega-\left\{\theta^{0}\right\}$. If moreover either $X$ is bounded or assumption 3 of [5] is satisfied, BIRNBAUM proves that $e_{0}(\alpha)$ is minimal complete, i.e. all tests in $e_{0}(\alpha)$ are admissible. As an example let $\underline{x}_{1}, \underline{x}_{2}, \ldots, \underline{x}_{k}$ denote $k$ independent normal random variables with expectations $\theta_{1}, \theta_{2}, \ldots, \theta_{\mathrm{k}}$ and unit variances; then BIRNBAUM's assumption 3 is satisfied and $e_{0}(\alpha)$ is a minimal complete class for testing $\theta=\theta^{0}$ against $\theta \neq \theta^{0}$. If the family (1.4.2) is not absolutely continuous but discrete (e.g. if $\lambda$ is counting measure), characterization of minimal complete classes is more difficult. Generalizing the work of BIRNBAUM in certain respects, C.M. STEIN 
in a paper [49] on the admissibility of HOTELLING's $\mathrm{T}^{2}$-test has given a sufficient condition for admissibility of a test of $\theta \in \Omega_{\mathrm{H}}$ against $\theta \in \Omega_{\mathrm{K}}$ for arbitrary $\Omega_{\mathrm{H}}$ and $\Omega_{\mathrm{K}}$ and exponential families with respect to arbitrary $\sigma$-finite measures $\lambda$. He has shown that a nonrandomized size- $\alpha$ test with closed convex acceptance region $A$ is admissible if for each supporting hyperplane of $\mathrm{A}$ there exist parameter points in $\Omega_{K}$ arbitrarily far out on some perpendicular to this hyperplane. However, in general these acceptance regions do not generate complete classes of tests.

MATTHES and TRUAX [35] considered composite null hypotheses of a particular kind: under $\mathrm{H}_{\mathrm{s}}$ some components, $\theta_{1}, \theta_{2}, \ldots, \theta_{\mathrm{S}}$ say, of the vector $\theta$ in (1.4.2) are specified while the remaining components of $\theta$ are unspecified nuisance parameters. In this situation it turns out that for any $\sigma$-finite measure $\lambda$ the totality of size- $\alpha$ tests $\delta$, which have acceptance regions $\{x \mid \delta(x)=0\}$ whose $\left(x_{s+1}, \ldots, x_{k}\right)$-sections are convex, constitute a complete class for testing $H_{S}$ against $K: \theta \in \Omega-\Omega_{\mathrm{H}}$ at level $\alpha$. By way of examples they have shown, however, that the tests in this class are not necessarily admissible, unless $s=1$.

Little seems to be known about essential completeness of the class $e_{0}(\alpha)$ if $\rho_{\Omega}$ is not some exponential family. To investigate this point we consider our original family of densities $p(x ; \theta)$ with respect to a $\sigma$-finite measure $\lambda$, where $X$ is a convex Borel set of $\mathbb{R}^{k}$. The index set $\Omega$ is arbitrary, but it is assumed that for different $\theta^{\prime}, \theta^{\prime \prime} \in \Omega$ the corresponding distributions are also different. It will also be assumed in this section that $p(x ; \theta)$ is positive for all $x \in X$ and $\theta \in \Omega$. This implies that the support of the distribution of $\underline{x}$ does not depend on $\theta$. If $\underline{x}$ is a discrete random variable, then $\lambda$ is a discrete measure and $p(x ; \theta)$ may be suitably defined for all points of $X$ with vanishing $\lambda$-measure.

A real-valued function $f$ defined on $X$ is said to be convex if for any $\left.x^{\prime}, x^{\prime}\left(x^{\prime} \neq x^{\prime}\right)^{\prime}\right)$ and any $0<\rho<1$

$$
f\left(\rho x^{\prime}+(1-\rho) x^{\prime} !\right) \leq \rho f\left(x^{\prime}\right)+(1-\rho) f\left(x^{\prime}\right) .
$$

The function $f$ is said to be strictly convex if the above inequality 
is always strict.

Suppose we wish to test the simple hypothesis

$$
\mathrm{H}_{0}: \theta=\theta^{0} \quad\left(\theta^{0} \in \Omega\right)
$$

against the alternative

$$
\mathrm{K}: \theta \in \Omega-\left\{\theta^{0}\right\} \text {. }
$$

Let $C_{0}(\alpha)$ and $B_{0}(\alpha)$ be defined as before and let $\beta_{0}^{*}(\alpha)$ denote the class of all essentially unique (in $\lambda$-measure) level- $\alpha$ Bayes tests of $\mathrm{H}_{0}$ against $\mathrm{K}$. The following theorem is an immediate generalization of BIRNBAUM's results.

\section{Theorem 1.4.1}

Suppose $\underline{x}=\left(\underline{x}_{1}, \ldots, \underline{x}_{k}\right)$ is distributed according to the density $p(x ; \theta)$, $\theta \in \Omega$, where $p(x ; \theta)$ is positive for all $x \in X$ and $\theta \in \Omega$. Lét $\theta^{0}$ denote an arbitrary point of $\Omega$. If for all $\theta \in \Omega$

$(1.4 \cdot 3)$

$$
p(x ; \theta) / p\left(x ; \theta^{0}\right)
$$

is either a convex function of $\mathrm{x}$ on $\mathrm{X}$ or a monotone function of a fixed linear combination of $\mathrm{x}_{1}, \ldots, \mathrm{x}_{\mathrm{k}}$ (the function being either non-decreasing for all $\theta$ or non-increasing for all $\theta$ ), then

$$
(1.4 .4) \quad B_{0}^{*}(\alpha) \subset e_{0}^{(\alpha)} \text { for all } \alpha \text {. }
$$

Moreover, of in addition all Bayes tests of $\mathrm{H}_{0}$ against $\mathrm{K}$ are essentially unique (in $\lambda$-measure), then $e_{0}(\alpha)$ is an essentially complete class for testing $\mathrm{H}_{0}$ against $\mathrm{K}$.

Proof:

Any Bayes test of $\mathrm{H}_{0}$ with respect to a prior distribution $\xi$ is equal a.e. [ $\lambda]$ to a test $\delta$ of the form

$$
(1.4 .5) \quad \delta(x)= \begin{cases}0 & \text { if } \int_{\Omega}\left[p(x ; \theta) / p\left(x ; \theta^{0}\right)\right] d \xi(\theta)<c \\ 1 & >c,\end{cases}
$$

where $c$ depends on the size of the test. Denote the integral appearing in $(1.4 .5)$ by $I(x)$. First let us assume that the functions (1.4.3) are 
convex on $X$. Then $I$ is also convex on $X$ and hence the sets $A_{-}=\{x \mid I(x)<c\}$ and $A_{+}=\{x \mid I(x) \leq c\}$ are both convex. If $\delta$ is essentially unique, either the set $\{x \mid I(x)=c\}$ has $\lambda$-measure zero or $\delta(x)=1$ a.e. $[\lambda]$ on this set or $\delta(x)=0$ a.e. $[\lambda]$ on this set. Hence either clos (A_) (in the first two cases) or clos $\left(A_{+}\right)$(in the last case) may serve as the closed convex set $A$ mentioned in (1.4.1). If (1.4.3) is a monotone function of $\sum a_{i} x_{i}$ for all $\theta$, then so is $I$ and the sets $A_{+}$and $A_{-}$are both half-spaces. The relation $(1.4 .4)$ is then proved as before. In case $B_{0}(\alpha)=B_{0}^{*}(\alpha)$, essential completeness of $C_{0}(\alpha)$ follows from the lemmata 1.3 .2 and 1.4 .1 . Q.E.D.

At first sight the conditions on the likelihood ratios (1.4.3) seem to be unnecessarily strong. However, it turns out that for an important family of distributions essential completeness of $e_{0}(\alpha)$ for all $\theta^{0} \in \Omega$ and all $0<\alpha<1$ implies that $\dot{\rho}_{\Omega}$ is either an exponential family or a family of distributions of an even simpler type. First we prove a basic lemma.

Lemma 1.4 .3

Let $\mathrm{X}=\mathbb{R}^{\mathrm{k}}$, let $\mathrm{p}(\mathrm{x} ; \theta)>0$ for all $\mathrm{x} \in \mathrm{X}$ and $\theta \in \Omega$ and let $\mathrm{p}\left(\mathrm{x} ; \theta^{\prime}\right) / \mathrm{p}\left(\mathrm{x} ; \theta^{\prime}{ }^{\prime}\right)$ be continuous in $\mathrm{x}$ for all $\hat{\theta}^{\prime}, \theta^{\prime} \in \in \Omega$. If for all $\theta^{\prime}, \theta^{\prime \prime} \in \Omega \quad\left(\theta^{\prime} \neq \theta^{\prime \prime}\right)$ and all real $c>0$ the sets

$$
\left\{x \mid p\left(x ; \theta^{\prime}\right) / p\left(x ; \theta^{\prime \prime}\right) \leq c\right\} \text { and }\left\{x \mid p\left(x ; \theta^{\prime}\right) / p\left(x ; \theta^{\prime \prime}\right)>c\right\}
$$

are convex or empty, then the function $p(x ; \theta)$ is of one of the following two forms:

(i) there exist functions $f$ and $q_{0}, q_{1}, \ldots, q_{k}$ such that

$$
p(x ; \theta)=f(x) \exp \left[\sum_{i=1}^{k} x_{i} q_{i}(\theta)+q_{0}(\theta)\right]
$$

identically in $\mathrm{x}$ for all $\theta \in \Omega$; or

(ii) there exist functions $f$ and $g$ and constants $a_{1}, a_{2}, \ldots, a_{k}$ such that

$$
p(x ; \theta)=f(x) g\left(\sum_{i=1}^{k} a_{i} x_{i} ; \theta\right)
$$

identically in $\mathrm{x}$ for all $\theta \in \Omega$; the function $\mathrm{g}$ is monotone (either 
non-decreasing or non-increasing) in its first argument.

Proof:

Let $\theta^{0}$ we some fixed point of $\Omega$ and let $\theta \epsilon \Omega$ be arbitrary, $\theta \neq \theta^{0}$.

Let

$$
c_{1}=\inf _{x}\left\{p(x ; \theta) / p\left(x ; \theta^{0}\right)\right\} \quad, c_{2}=\sup _{x}\left\{p(x ; \theta) / p\left(x ; \theta^{0}\right)\right\},
$$

and consider an arbitrary $c \in\left(c_{1}, c_{2}\right)$. Since the sets $A_{1}=\left\{x \mid p(x ; \theta) / p\left(x ; \theta^{0}\right) \leq c\right\}$ and $A_{2}=\left\{x \mid p(x ; \theta) / p\left(x ; \theta^{0}\right)>c\right\}$ are non-empty and convex and the set $A_{2}$ is open by the continuity of $p(x ; \theta) / p\left(x ; \theta^{0}\right)$, there exists a hyperplane $H_{c}$ separating $A_{1}$ and $A_{2}$ (cf. H.G. EGGLESTON [15] section 1.7). It is easily shown by a continuity argument that $p(x ; \theta) / p\left(x ; \theta^{0}\right)$ is identically equal to $c$ on $H_{c}$. For different $c \in\left(c_{1}, c_{2}\right)$ the hyperplanes $H_{c}$ are parallel, because they cannot have common points in $\mathrm{X}$. Let

$$
\sum_{i=1}^{k} a_{i}\left(\theta, \theta^{0}\right) x_{i}=a_{0}\left(c ; \theta, \theta^{0}\right)
$$

be the equation of $\mathcal{H}_{c}$; the coefficients $a_{i}$ are assumed to be normalized: $\sum_{i=1}^{k} a_{i}^{2}\left(\theta, \theta^{0}\right)=1$. The left-hand member of $(1.4 .9)$ will be denoted by $L\left(x ; \theta, \theta^{0}\right)$. The function $p(x ; \theta) / p\left(x ; \theta^{0}\right)$ depends on $x$ only through $L\left(x ; \theta, \theta^{0}\right)$; this may be expressed by writing

$$
p(x ; \theta) / p\left(x ; \theta^{0}\right)=g\left(L\left(x ; \theta, \theta^{0}\right) ; \theta, \theta^{0}\right),
$$

where the function $g\left(. ; \theta, \theta^{0}\right)$ is continuous and monotone (either non-decreasing or non-increasing) and not constant, since $P_{\theta}$ and $P_{\theta} 0$ are different.

To any $\theta \in \Omega\left(\theta \neq \theta^{0}\right)$ corresponds a linear form $L\left(x ; \theta, \theta^{0}\right)$. We define the relation $\stackrel{\mathrm{L}}{=}$ on $\Omega-\left\{\theta^{0}\right\}$ as follows :

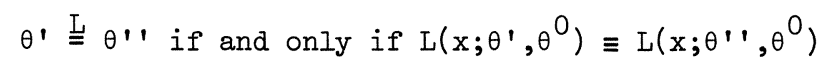

$\left(\theta^{0}\right.$ fixed). The relation $\stackrel{L}{=}$ is obviously an equivalence relation which defines equivalence classes in $\Omega-\left\{\theta^{0}\right\}$. Let $\theta$ denote the set of all equivalence classes. We consider two cases.

(i) The set $\theta$ contains at least two elements. Let $\theta \in \Omega\left(\theta \neq \theta^{0}\right)$ be arbitrary and let $\theta^{\prime} \in \Omega\left(\theta^{\prime} \neq \theta^{0}\right)$ belong to another equivalence class than $\theta$. Since

we find

$$
p(x ; \theta) / p\left(x ; \theta^{0}\right) \equiv\left[p(x ; \theta) / p\left(x ; \theta^{\prime}\right)\right]\left[p\left(x ; \theta^{\prime}\right) / p\left(x ; \theta^{0}\right)\right]
$$




$$
g\left(L\left(x ; \theta, \theta^{0}\right) ; \theta, \theta^{0}\right) \equiv g\left(L\left(x ; \theta, \theta^{\prime}\right) ; \theta, \theta^{\prime}\right) g\left(L\left(x ; \theta^{\prime}, \theta^{0}\right) ; \theta^{\prime}, \theta^{0}\right),
$$

where $L\left(x ; \theta, \theta^{0}\right)$ and $L\left(x ; \theta^{\prime}, \theta^{0}\right)$ are linearly independent. The three linear forms $L\left(x ; \theta, \theta^{0}\right), L\left(x ; \theta^{\prime}, \theta^{0}\right)$ and $L\left(x ; \theta, \theta^{\prime}\right)$ are not linearly independent. For if they were linearly independent, the third linear form may vary from $-\infty$ to $+\infty$ for fixed values of the first two linear forms, implying that $\mathrm{g}\left(\cdot ; \theta^{\prime}, \theta^{\prime}\right)$ is constant which is impossible. Thus $\mathrm{L}\left(\mathrm{x} ; \theta^{\prime}, \theta^{\prime}\right)$ is a linear combination of the two other linear forms. Taking logarithms and writing $h=\log g, L\left(x ; \theta, \theta^{0}\right)=y$ and $L\left(x ; \theta^{\prime}, \theta^{0}\right)=z$, we obtain

$$
h\left(y ; \theta, \theta^{0}\right) \equiv h\left(a^{\prime} y+b^{\prime} z ; \theta, \theta^{\prime}\right)+h\left(z ; \theta^{\prime}, \theta^{0}\right),
$$

where $a^{\prime}$ and $b^{\prime}$ are appropriate constants. It is easily seen that $a^{\prime} b^{\prime} \neq 0$, because otherwise one of the functions $h$ would again be identically equal to a constant. Putting $a^{\prime} y+b^{\prime} z=w, a=1 / a^{\prime}, b=-b^{\prime} / a^{\prime},(1.4 .12)$ is more conveniently expressed as

$$
h\left(a w+b z ; \theta, \theta^{0}\right) \equiv h\left(w ; \theta, \theta^{0}\right)+h\left(z ; \theta^{\prime}, \theta^{0}\right) .
$$

Moreover, substitution of $\mathrm{w}=0$ or $\mathrm{z}=0$ respectively in $(1.4 .13)$ yields

$$
\begin{aligned}
& h\left(w ; \theta, \theta^{\prime}\right) \equiv h\left(a w ; \theta, \theta^{0}\right)-h\left(0 ; \theta^{\prime}, \theta^{0}\right), \\
& h\left(z ; \theta^{\prime}, \theta^{0}\right) \equiv h\left(b z ; \theta, \theta^{0}\right)-h\left(0 ; \theta, \theta^{\prime}\right),
\end{aligned}
$$

and thus $(1.4 .13)$ may be written as

$$
\begin{aligned}
h\left(a w+b z ; \theta, \theta^{0}\right) \equiv & h\left(a w ; \theta, \theta^{0}\right)+h\left(b z ; \theta, \theta^{0}\right)+ \\
& -h\left(0 ; \theta^{\prime}, \theta^{0}\right)-h\left(0 ; \theta, \theta^{\prime}\right) .
\end{aligned}
$$

Defining $\tilde{h}\left(y ; \theta, \theta^{0}\right)=h\left(y ; \theta, \theta^{0}\right)-h\left(0 ; \theta, \theta^{0}\right)$, we find

$$
\tilde{h}\left(a w+b z ; \theta, \theta^{0}\right) \equiv \tilde{h}\left(a w ; \theta, \theta^{0}\right)+\tilde{h}\left(b z ; \theta, \theta^{0}\right) \text {. }
$$

Since $\tilde{h}$ is continuous, the identity $(1.4 .14)$ implies

$$
\tilde{\mathrm{h}}\left(\mathrm{y} ; \theta, \theta^{0}\right) \equiv \mathrm{d}\left(\theta, \theta^{0}\right) \mathrm{y}
$$

for some non-zero $d\left(\theta, \theta^{0}\right)$ (cf.H. HAHN and A. ROSENTHAL [20]th.9.3.1) and thus

$$
\mathrm{h}\left(\mathrm{y} ; \theta, \theta^{0}\right) \equiv d\left(\theta, \theta^{0}\right) \mathrm{y}+\mathrm{a}_{0}\left(\theta, \theta^{0}\right),
$$

where $a_{0}\left(\theta, \theta^{0}\right)=h\left(0 ; \theta, \theta^{0}\right)$. But $(1 \cdot 4 \cdot 15)$ implies 


$$
p(x ; \theta) \equiv p\left(x ; \theta^{0}\right) \exp \left[\sum_{i=1}^{k} a_{i}\left(\theta, \theta^{0}\right) d\left(\theta, \theta^{0}\right) x_{i}+a_{0}\left(\theta, \theta^{0}\right)\right] \text {. }
$$

Since $\theta^{0}$ was fixed, we may write $q_{i}(\theta)=a_{i}\left(\theta, \theta^{0}\right) d\left(\theta, \theta^{0}\right), i=1,2, \ldots, k$ and $q_{0}(\theta)=a_{0}\left(\theta, \theta^{0}\right)$. Defining the functions $q_{0}, q_{1}, \ldots, q_{k}$ to be zero for $\theta=\theta^{0}$ we obtain (1.4.6).

(ii) The set $\theta$ contains only one element. In this case (1.4.7) immediately follows from $(1.4 \cdot 10)$.

Q.E.D.

\section{Theorem 1.4.2}

Let $X=\mathbb{R}^{k}$ and let $\lambda$ be equivalent with Lebesgue measure on $X$. Let $\underline{x}=\left(\underline{x}_{1}, \ldots, \underline{x}_{k}\right)$ be distributed according to the density $p(x ; \theta), \theta \in \Omega$, where $p(x ; \theta)$ is positive for all $x \in X$ and $\theta \in \Omega$ and $p\left(x ; \theta^{\prime}\right) / p\left(x ; \theta^{\prime \prime}\right)$ is continuous in $x$ for all $\theta^{\prime}, \theta^{\prime \prime} \in \Omega$. If for all $0<\alpha<1$ and all $\theta^{0} \in \Omega$ the class $e_{0}(\alpha)$ is essentially complete for testing $H_{0}: \theta=\theta^{0}$ against $K: \theta \in \Omega-\left\{\theta^{0}\right\}$, then the densities $p(x ; \theta)$ are of one of the two forms $(i)$ or (ii) of lemma 1.4.3.

Proof:

If $e_{0}(\alpha)$ is essentially complete, it certainly contains a version of each essentially unique most powerful test of $\mathrm{H}_{0}$ against a simple alternative. Let $\theta$ and $\theta^{0}\left(\theta \neq \theta^{0}\right)$ be arbitrary points of $\Omega$. Consider the most powerful test of $\theta^{0}$ against $\theta$

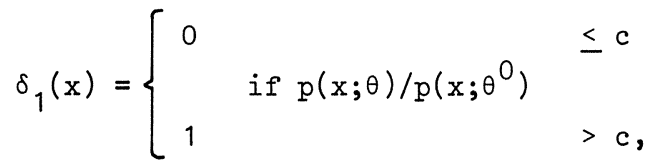

where $c$ is an arbitrary positive number, and let $\alpha$ denote the size of this test. The critical function $\delta_{2}(x) \equiv 1-\delta_{1}(x)$ defines a most powerful test of $\theta$ against $\theta^{0}$. First we assume that $c_{1}<c<c_{2}$, where $c_{1}$ and $c_{2}$ are defined in (1.4.8); it follows that $0<\alpha<1$ in this case. Since both $\delta_{1}$ and $\delta_{2}$ are essentially unique most powerful tests, they must be equal a.e. [ $\lambda]$ to tests with convex acceptance regions; let us denote these convex acceptance regions by $A_{1}$ and $A_{2}$ respectively. We shall show that the sets $\left\{x \mid p(x ; \theta) / p\left(x ; \theta^{0}\right) \leq c\right\}$ and $\left\{x \mid p(x ; \theta) / p\left(x ; \theta^{0}\right)>c\right\}$ are both convex. It then follows by a limiting argument that these sets are also convex or empty for values of $c$ not in $\left(c_{1}, c_{2}\right)$. The proof of the theorem is then completed by application of lemma 1.4.3.

It is easily verified that any common point of $A_{1}$ and $A_{2}$ is a boundary point of both $A_{1}$ and $A_{2}$. Hence $\operatorname{int}\left(A_{1}\right)$ and $A_{2}$ are disjoint and there exists a hyperplane 
$H_{c}$ separating $\operatorname{int}\left(A_{1}\right)$ and $A_{2}$. By a continuity argument $p(x ; \theta) / p\left(x ; \theta^{0}\right)=c$ for all $x \in H_{c}$. The hyperplane $H_{c}^{2}$ separates $\mathbb{R}^{k}$ into two open half-spaces; let $A_{1}^{*}$ be the open half-space containing $\operatorname{int}\left(A_{1}\right)$ and let $A_{2}^{*}$ be the other open half-space. Then $p(x ; \theta) / p\left(x ; \theta^{0}\right) \leq c$ for all $x \in \operatorname{clos}\left(A_{1}^{*}\right)$ by the continuity of $p(x ; \theta) / p\left(x ; \theta^{0}\right)$. Moreover, $p(x ; \theta) / p\left(x ; \theta^{0}\right)>c$ for all $x \in A_{2}^{*}$. For suppose there exists a point $x^{0} \in A_{2}^{*}$ such that $p\left(x^{0} ; \theta\right) / p\left(x^{0} ; \theta^{0}\right)=c_{0} \leq c$. The continuity of the likelihood ratio implies that $c_{0}=c$. Let $D$ be the set of points between $Y_{c}$ and the hyperplane $H^{0}$ through $x^{0^{0}}$ and parallel to $H_{c}$. Consider $\sup \left\{p(x ; \theta) / p\left(x ; \theta^{0}\right) \mid x \in D\right\}=c^{\prime}$. If $c^{\prime}=c$ we have a contradiction with the definition of $A_{1}$ and $A_{1}^{*}$. If $c^{\prime}>c$, let $c^{\prime \prime}=\frac{1}{2}\left(c+c^{\prime}\right)$ and construct the hyperplane $\mathcal{H} c^{\prime}$, corresponding to $c^{\prime \prime}$ in a similar way as $\mathscr{H}_{c}$. Then $\mathcal{H}_{c^{\prime}}$, is parallel to $\mathcal{H}_{c}{ }^{c}$ and separates $A_{1}^{*}$ and $x^{0}$, which is also impossible. Hence a point $x^{0}$ with the property stated does not exist. It follows that $\left\{x \mid p(x ; \theta) / p\left(x ; \theta^{0}\right) \leq c\right\}=\operatorname{clos}\left(A_{1}^{*}\right)$ and $\left\{x \mid p(x ; \theta) / p\left(x ; \theta^{0}\right)>c\right\}=A_{2}^{*}$, and thus both sets are convex.

Q.E.D.

If $\underline{x}_{1}, \underline{x}_{2}, \ldots, \underline{x}_{k}$ are independent, an analysis similar to the proof of lemma 1.4 .3 shows that in case (ii) the density $p(x ; \theta)$ can be written in the form

$$
p(x ; \theta)=f^{*}(x) \exp \left[b(\theta) \sum_{i=1}^{k} a_{i} x_{i}+b_{0}(\theta)\right],
$$

i.e. the family of distributions $(1.4 .7)$ is an exponential family with a single sufficient statistic $\sum a_{i} x_{i}$.

Although theorem 1.4.2 only applies to a rather resticted family of distributions, it indicates nevertheless that the essential completeness property of tests with a.e. convex acceptance regions cannot easily by extended to other than exponential families if the value of $\theta$ specified by the hypothesis is an arbitrary interior point of the parameter space.

We now consider tests of a simple hypothesis

$$
\mathrm{H}_{0}: \theta=\theta^{0}
$$

against one-sided alternatives

$$
K^{\prime}: \theta \cdot \geq \theta^{0}, \theta \in \Omega,
$$

in a family $\rho_{\Omega}$ of distributions with densities $\mathrm{p}(\mathrm{x} ; \theta)$ with respect to a $\sigma$-finite measure $\lambda ; \mathrm{X}$ and $\Omega$ are assumed to be Borel sets of $\mathbb{R}^{\mathrm{k}}$ and $\mathbb{R}^{r}$ respectively (in most applications $r=k$ ). 
We say that a real-valued function $\psi$ of vector argument $\mathrm{x}$ defined on $\mathrm{X} \in \mathbb{R}^{\mathrm{k}}$ is non-decreasing in $\mathrm{x}$ if $\mathrm{x}^{\prime} \leq \mathrm{x}\left(\mathrm{x}^{\prime}, \mathrm{x} \in \mathrm{X}\right)$ implies $\psi\left(\mathrm{x}^{\prime}\right) \leq \psi(\mathrm{x})$. If in addition $x^{\prime}<x$ (the inequality holding for all components) implies $\psi\left(x^{\prime}\right)<\psi(x)$, then $\psi$ is said to be increasing in $x$.

We say that a closed subset $A$ of $\mathbb{R}^{k}$ is monotone if $x \in A$ and $x^{\prime} \leq$. $x$ imply $x^{\prime} \in A$, If $B$ is a closed subset of $\mathbb{R}^{k}$, the closed monotone set $\{x \mid x \leq y, y \in B\}$ is called the monotone extension of $B$.

A test $\delta$ is said to be monotone if for some closed monotone subset $A$ of $\mathbb{R}^{k}$

$$
\delta(x)= \begin{cases}0 & \text { if } x \in X \cap \operatorname{int}(A) \\ 1 & \text { if } x \in X-A .\end{cases}
$$

The class of all tests which are equal a.e. $[\lambda]$ to any monotone size- $\alpha$ test of $\theta=\theta^{Q}$ will be denoted by $\mathcal{M}_{0}(\alpha)$. We call $\mathcal{M}_{0}(\alpha)$ the class of a.e. monotone size- $\alpha$ tests of $\theta=\theta^{0}$, or the class of size- $\alpha$ tests of $\theta=\theta^{0}$ with a.e. monotone acceptance regions.

If $\psi$ is an increasing function of $x$ on $X$ and $c$ is an arbitrary constant, a test $\delta^{\prime}$ satisfying

$$
\delta^{\prime}(x)= \begin{cases}0 & \text { if } \psi(x)<c \\ 1 & \text { if } \psi(x)>c\end{cases}
$$

is a monotone test. The monotone extension of the set clos $(\{x \mid \psi(x) \leq c\})$ may serve as the closed monotone set $A$ appearing in (1.4.18). Conversely, given any monotone test $\delta$ there exist an increasing function $\psi$ and a constant $c$ such that the test $\delta$ satisfies (1.4.19). To show this, let A be some closed monotone set, let $c=0$ and define

$$
\psi_{A}(x)=d^{*}(A, x)-d^{*}\left(\mathbb{R}^{k}-A, x\right),
$$

where $d^{*}(B, x)=\inf \left\{d^{*}(y, x) \mid y \in B\right\}$ and $d^{*}(y, x)$ denotes the Euclidean distance of $\mathrm{x}$ and $\mathrm{y}$. Obviously $\psi_{A}(\mathrm{x})<0$ for $\mathrm{x} \in \operatorname{int}(\mathrm{A})$ and $\psi_{A}(\mathrm{x})>0$ for $\mathrm{x} \in \mathrm{X}-\mathrm{A}$. The monotonicity of A ensures that $\psi_{A}$ is indeed increasing in $x$ and hence the function $\psi_{A}$ has the required properties.

Moreover, if for some function $\psi$ a family of tests is defined by (1.4.19) for all sizes $\alpha$ by varying $c$, and if all these tests are monotone, then $\psi$ is non-decreasing in $\mathrm{x}$ on $\mathrm{x}$.

Let $S(q)$ denote the solid hypersphere in $\mathbb{R}^{\mathrm{k}}$ whose centre is the origin and 
whose radius is $q$, and let the Hausdorff distance of two subsets $B_{1}$ and $B_{2}$ of $s(q)$ be denoted by

$$
d\left(B_{1}, B_{2}\right) \stackrel{\text { def }}{=} \text { inf }\left\{\varepsilon \mid B_{1} \subset U\left(B_{2}, \varepsilon\right), B_{2} \subset U\left(B_{1}, \varepsilon\right)\right\}
$$

where $U(B, \varepsilon)$ denotes the $\varepsilon$-neighborhood of $B$.

We say that a closed subset $B$ of $S(q)$ is $S(q)$-monotone if $x \in B$ and $x^{\prime} \leq x$, $\mathrm{x}^{\prime} \in \mathrm{S}(\mathrm{q})$ imply $\mathrm{x}^{\prime} \in \mathrm{B}$.

To obtain the analogue of lemma 1.4.1 for the class $\mathcal{M}_{0}(\alpha)$ we use the same method of proof as MATTHES and TRUAX [35]. Instead of BLASCHKE's selection theorem (cf. EGGLESTON [15]th.32) we need the following more general result, which may e.g. be found in P. ALEXANDROFF and H. HOPF [ 1$], \mathrm{p} .115$.

Lemma 1.4 .4 .

Let $B_{1}, B_{2}, B_{3}, \ldots$ denote an infinite sequence of closed non-empty subsets of $S(q)$. Then there exists a subsequence $\left\{B_{n_{i}}\right\}$ such that

$$
\lim _{i \rightarrow \infty} d\left(B_{n_{i}}, B\right)=0
$$

where $B$ is a closed non-empty set.

\section{Corollary 1.4 .1}

Let $B_{1}, B_{2}, B_{3}, \ldots$ denote an infinite sequence of closed $S(q)$ - monotone non-empty subsets of $S(q)$. Then there exists a subsequence $\left\{B_{n_{i}}\right.$ tand a closed $S(q)$ - monotone non-empty set $B$ such that

$$
\lim _{i \rightarrow \infty} d\left(B_{n_{i}}, B\right)=0
$$

Proof:

Apply lemma 1.4.4. The limiting set $B$ is $S(q)$-monotone because a closed limit (in the sense of the metric $(1.4 .20)$ ) of a sequence of closed $S(q)$ - monotone sets is always $\mathrm{S}(\mathrm{q})$ - monotone.

Q.E.D.

Starting from this corollary we may proceed as in the proof of theorem 2.1. in [35], with only minor modifications, to obtain the analogue of lemma 1.4.1. 
Lemma 1.4 .5

For any $\sigma$-finite measure $\lambda$ dominating the family $\rho_{\Omega}$ the class $\mathcal{M}_{0}(\alpha)$ is closed in the topology of weak convergence.

A family of densities $p(x ; \theta), \theta \in \Omega, x \in X$, with respect to a $\sigma$-finite measure $\lambda$ is said to have monotone likelihood ratio (MLR) in the vector $x$ if for all $\theta^{\prime} . \geq \theta^{\prime \prime}$ the ratio $\mathrm{p}\left(\mathrm{x} ; \theta^{\prime}\right) / \mathrm{p}\left(\mathrm{x} ; \theta^{\prime}{ }^{\prime}\right)$ is non-decreasing in $\mathrm{x}$ on $\mathrm{X}$. If this ratio is increasing in $x$ on $X$, we say that the family has strict MLR in $x$. (Strict) MLR of one-parameter families of densities of scalar random variables is similarly defined.

\section{Theorem 1.4.3}

Let $\underline{x}=\left(\underline{x}_{1}, \ldots, \underline{x}_{k}\right)$ have density $p(x ; \theta), \theta \in \Omega$, with respect to a $\sigma$-finite measure $\lambda$ defined on $X \subset \mathbb{R}^{\mathrm{k}}$, and let $\mathrm{p}(\mathrm{x} ; \theta)$ be positive for all $\mathrm{x} \in \mathrm{X}$ and $\theta \in \Omega$. If the densities $p(x ; \theta)$ have strict MLR in the vector $x$ a.e. $[\lambda]$ (where the exceptional $\lambda$-nullsets do not depend on the parameter $\theta$ in the numerator of the likelihood ratio), then the class $\mathcal{M}_{0}(\alpha)$ of a.e. monotone size- $\alpha$ tests is essentially complete for testing $\theta=\theta^{0}$ against one-sided alternatives $\theta . \geq \theta^{0}$ at level $\alpha$ for all $\theta^{0} \epsilon \Omega$ and all $0<\alpha<1$. Conversely, if $\mathcal{M}_{0}(\alpha)$ is essentially complete for testing any $\theta=\theta^{0} \epsilon \Omega$ against $\theta . \geq \theta^{0}$ for all $0<\alpha<1$, then the densities $p(x ; \theta)$ have MLR in $\mathrm{x}$ a.e. $[\lambda]$.

Proof:

Suppose $p(x ; \theta)$ has strict MLR in $x$ a.e. $[\lambda]$. Any Bayes test of $\theta=\theta^{0}$ against $\theta \cdot \geq \theta^{0}$ is equal a.e. $[\lambda]$ to a test $\delta$ of the form

$$
\delta(x)=\left\{\begin{array}{lll}
0 & \text { if } \int_{\theta . \geq 0^{0}}\left[\mathrm{p}(\mathrm{x} ; \theta) / \mathrm{p}\left(\mathrm{x} ; \theta^{0}\right)\right] \mathrm{d} \xi(\theta) & <\mathrm{c} \\
1 & >c,
\end{array}\right.
$$

where $\xi$ is a prior distribution and $c$ depends on the size of the test. The MLR characte: of $p$ implies that the integral is increasing in $x$ a.e. $[\lambda]$. Hence $\mathcal{B}_{0}(\alpha)$ c $\mathcal{M}_{0}(\alpha)$ and the essential completeness of $\mathcal{M}_{0}(\alpha)$ is a consequence of the lemmata 1.3 .2 and 1.4 .5 .

Conversely, suppose $M_{0}(\alpha)$ is essentially complete for all $\theta^{0} \epsilon \Omega$ and all $\alpha$. Then any essentially unique size- $\alpha$ Bayes test of any $\theta=\theta^{0}$ against $\theta \geq \theta^{0}$ is contained in $\mathcal{M}_{0}(\alpha)$. Any Bayes test of $\theta=\theta^{0}$ against a simple alternative $\theta=\theta^{\prime} \cdot \geq \theta^{0}$ satisfies a.e. $[\lambda]$ 


$$
\delta(x)=\left\{\begin{array}{lll}
0 & & <c \\
1 & \text { if } p\left(x ; \theta^{\prime}\right) / p\left(x ; \theta^{0}\right) & >c,
\end{array}\right.
$$

where $c$ depends on the size of the test. Such tests are essentially unique if $\delta(x)=0($ or 1$)$ on the set where $p\left(x ; \theta^{\prime}\right) / p\left(x ; \theta^{0}\right)=c$. Noting that this ratio must be non-decreasing in $x$ a.e. $[\lambda]$ since $\delta$ is a.e. monotone for all $c$, the proof is complete.

Q.E.D.

This theorem is related to a result of BIRNBAUM [4], stating that any monotone test is Bayes against some simple alternative. However, BIRNBAUM starts from a much larger family of distributions and hence theorem 1.4 .3 does not follow from his result.

If $\lambda$ is a product measure and $\underline{x}_{1}, \underline{x}_{2}, \ldots, \underline{x}_{k}$ are independent with densities $p_{i}\left(x_{i} ; \theta_{i}\right)(i=1,2, \ldots, k)$, strict MLR in $x$ of the family $p(x ; \theta)=\prod_{i} p_{i}\left(x_{i} ; \theta_{i}\right)$ is equivalent with strict MLR of each family $p_{i}\left(x_{i} ; \theta_{i}\right)$ in $x_{i}, i=1,2, \ldots, k$.

Combining the theorems 1.4 .1 and 1.4 .3 we obtain sufficient conditions for essential completeness of the class of tests with a.e. convex and monotone acceptance regions for testing a hypothesis $\theta=\theta^{0}$ against one-sided alternatives $\theta . \geq \theta^{0}$ at all significance levels $\alpha$. Essential completeness of this class was stated without proof by R. SCHWARZ [47] for exponential families. BIRNBAUM [5] gave a stronger result for independent normal random variables.

Lemma 1.4 .6 (A. BIRNBAUM)

Let $\underline{x}_{1}, \underline{x}_{2}, \ldots, \underline{x}_{k}$ denote $k$ independent random variables, where $\underline{x}_{i}$ is $N\left(\mu_{i}, 1\right)$ distributed $(i=1,2, \ldots, k)$. The class of size- $\alpha$ tests with a.e. convex and monotone acceptance regions is minimal complete for testing $\mu=\left(\mu_{1}, \ldots, \mu_{k}\right)=0$ against $\mu . \geq 0$ for all $0<\alpha<1$.

\subsection{TOTALLY POSITIVE FUNCTIONS}

In subsequent chapters we shall occasionally apply the variation-diminishing property of integrals with totally positive kernels. In this section we list the necessary definitions and results that will be used in the sequel. The theory has largely been developed by I.J. SCHOENBERG and S. KARLIN. For a detailed exposition of the theory we refer to KARLIN's book [25].

Let $K(y, z)$ denote a real function defined on $Y \times Z$ where $Y$ and $Z$ are subsets of $\mathbb{R}^{1}$. The function $K$ is said to be totally positive of order $n\left(T P_{n}\right)$ on $Y \times Z$ if for all $1 \leq m \leq n$ 
$(1.5 .1)$

$$
\mathrm{K}\left(\begin{array}{l}
\left.\mathrm{y}_{1}, \ldots, \mathrm{y}_{\mathrm{m}}\right) \\
\mathrm{z}_{1}, \ldots, \mathrm{z}_{\mathrm{m}}
\end{array}\right) \quad\left|\begin{array}{ccc}
\mathrm{K}\left(\mathrm{y}_{1}, \mathrm{z}_{1}\right) & \ldots \mathrm{K}\left(\mathrm{y}_{1}, \mathrm{z}_{\mathrm{m}}\right) \\
\vdots & \vdots \\
\mathrm{K}\left(\mathrm{y}_{\mathrm{m}}, \mathrm{z}_{1}\right) & \ldots & \vdots \\
\mathrm{K}\left(\mathrm{y}_{\mathrm{m}}, \mathrm{z}_{\mathrm{m}}\right)
\end{array}\right| \geq 0
$$

for all $\mathrm{y}_{1}<\mathrm{y}_{2}<\ldots<\mathrm{y}_{\mathrm{m}}$ and $\mathrm{z}_{1}<\mathrm{z}_{2}<\ldots<\mathrm{z}_{\mathrm{m}}, \mathrm{y}_{\mathrm{i}} \in \mathrm{Y}, \mathrm{z}_{\mathrm{i}} \in \mathrm{Z}$. $\mathrm{K}$ is said to be strictly $\mathbb{T P}_{\mathrm{n}}$ if the inequalities $(1.5 .1)$ are always strict. $\mathrm{K}$ is said to be (strictly) TP if $K$ is (strictly) $T_{n}$ for $n=1,2, \ldots$.

If a $\mathbb{T P}_{n}$ function $K$ may be written in the form $K(y, z)=f(y-z)$, where both $Y$ and $Z$ are the real line, then $f$ is said to be a Polya frequency function of order $n\left(P F{ }_{n}\right)$. If $K$ is $T_{n}, K(y, z)=f(y-z)$ but $Y$ and $Z$ are the set of all integers, then $f$ is said to be a Pólya frequency sequence of order $n$ (also abbreviated $\mathrm{PF}_{\mathrm{n}}$ ).

Let $K(y, z)$ be $n-1$ times continuously differentiable with respect to $y$ for each $z \in Z$, where $Y$ is an open interval. Then $K$ is said to be extended totally positive of order $n$ in $y$ - written $\operatorname{ETP}_{n}(y)-$ if for all $1 \leq m \leq n$

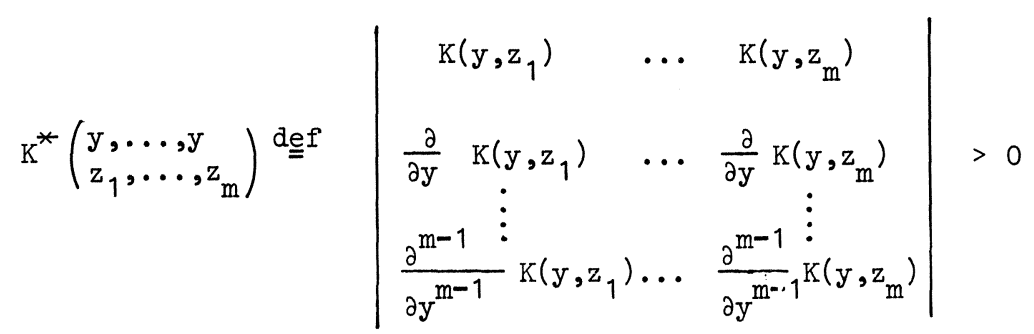

for all $y \in Y$ and $z_{1}<z_{2}<\ldots<z_{m}, z_{i} \in Z$.

If $Y$ and $Z$ are both open intervals and $K(y, z)$ has continuous partial derivatives of order $2 n-2$, then $K$ is said to be extended totally positive of order $n$ in both variables - written $\operatorname{ETP}_{n}$ - if for all $1 \leq m \leq n$

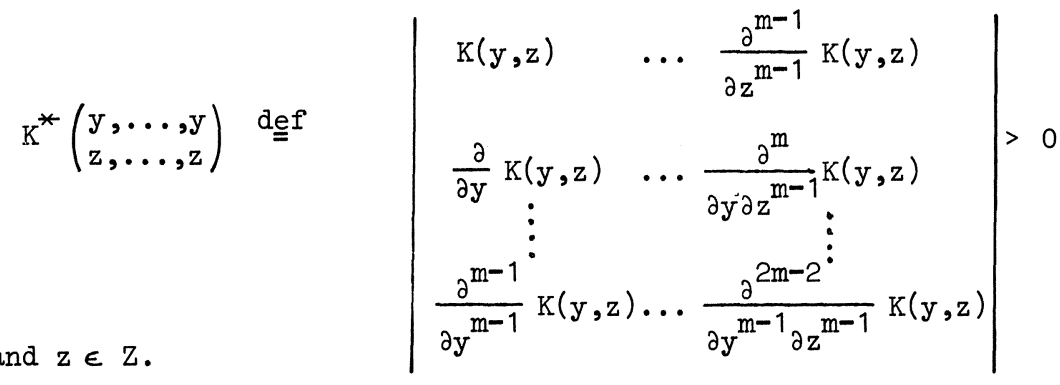

for all $\mathrm{y} \in \mathrm{Y}$ and $\mathrm{z} \in \mathrm{Z}$. 
Total positivety of a function is often established by means of the following lemma (cf. [25] th.2.2.4 and th.2.2.6).

Lemma 1.5 .1 (S. KARLIN)

If $K(y, z)$ is $\operatorname{ETP}_{n}$, then $K(y, z)$ is also $\operatorname{ETP}_{n}(y)$. If $K(y, z)$ is $\operatorname{ETP}_{n}(y)$, then $K(y, z)$ is also strictly $\mathrm{TP}_{\mathrm{n}}$.

In the particular case where $n=2$ we have (cf. [25] th.4.1.5)

Lemma 1.5 .2 (S. KARLIN)

Let $K(y, z)>0 \quad(y \in Y, z \in Z, Y$ and $Z$ are both intervals), and assume $K$ to be twice differentiable. Then $\mathrm{K}$ is $\mathrm{TP}_{2}$ if and only if

$$
\frac{\partial^{2}}{\partial y \partial z} \log K(y, z) \geq 0 \quad \text { for all } y \in Y, z \in Z \text {. }
$$

This lemma implies that a differentiable positive function $f$ defined on $\mathbb{R}^{1}$ is $\mathrm{PF}_{2}$ if and only if $\mathrm{d}^{2} \log \mathrm{f}(\mathrm{y}) / \mathrm{dy}^{2} \leq 0$ for all $\mathrm{y}$.

As an example of TP functions we mention

$$
K(y, z) \stackrel{d e f}{=} a(y) b(z) \exp [\alpha(y) \beta(z)],
$$

where $a(y)>0, b(z)>0$ and $\alpha(y)$ and $\beta(z)$ are non-decreasing. $K$ is strictly TP if $\alpha(y)$ and $\beta(z)$ are strictly increasing. Hence the densities of one-parameter exponential families are TP.

We note that the $\mathbb{T P}_{2}$ property of one-parameter densities $p(x ; \theta)$ is equivalent to monotone likelihood ratio (MLR) of $p(x ; \theta)$ in $x$.

Let $h$ denote a real function defined on $z$. The number of sign changes $S(h)$ of $h$ is equal to $n$ if for $m=n$ a sequence $z_{1}<z_{2}<\ldots<z_{m}, z_{i} \in z$, exists such that either $(-1)^{j+1} h\left(z_{j}\right)>0(j=1,2, \ldots, m+1)$ or $(-1)^{j} h\left(z_{j}\right)>0(j=1,2, \ldots, m+1)$, but for $m=n+1$ such a sequence does not exist. In the first case we say that $h$ changes sign in the order (+-+-...) for increasing $z$, in the second case that $h$ changes sign in the order $(-+-+\ldots)$ for increasing $z$.

We now state the fundamental variation-diminishing property (cf. [25] th.5.3.1).

Lemma 1.5 .3 (S. KARLIN)

Let $\mathrm{K}$ be $\mathrm{TP}_{\mathrm{n}}$ on $\mathrm{Y} \times \mathrm{Z}$, let $\lambda$ be a $\sigma$-finite measure on $\mathrm{Z}$, and let $\mathrm{g}(\mathrm{y})$ be $\operatorname{defined}$ by the absolutely convergent integral 


$$
g(y) \stackrel{\text { def }}{=} \int K(y, z) h(z) d \lambda(z) \quad(y \in Y) .
$$

If $S(h) \leq n-1$, then $S(g) \leq S(h)$; if $S(g)=S(h)$, the functions $h$ and $g$ change sign in same order.

The following stronger form of lemma 1.5 .3 is also important (cf. [25]th.5.3.2).

Lemma 1.5 .4 (S. KARLIN)

Let $\mathrm{K}$ be $\mathrm{n}-1$ times continuously differentiable with respect to $\mathrm{y}$ for all $\mathrm{z} \in \mathrm{Z}$, where $Y$ is an open interval, and let $K$ be $\operatorname{ETP}_{n}(y)$ on $Y \times Z$. Let $g(y)$ be defined by (1.5.4) and assume that differentation of $g$ may be performed $n-1$ times under the integral sign appearing in (1.5.4). If $S(h) \leq n-1$, then $g$ has at most $S(h)$ zero's counting multiplicities, unless $h$ vanishes a.e. $[\lambda]$ on $z$.

We shall also need a theorem due to B. EFRON [14].

Lemma 1.5 .5$. (B. EFRON)

Let $\underline{x}_{1}, \underline{x}_{2}, \ldots, \underline{x}_{k}$ be independent random variables with densities $f_{1}(x), f_{2}(x), \ldots$ $\ldots, f_{k}(x)$ respectively, and let $\underline{s}=\sum_{i=1}^{k} \underline{x}_{i}$. The measure $\lambda$ is assumed to be either Lebesgue measure or counting measure, and the functions $f_{i}$ are assumed to be $\mathrm{PF}_{2}$. Let $\mathrm{F}$ be a real measurable function of $\mathrm{k}$ arguments that is non-decreasing in each of its arguments. Then the conditional expectation

$$
E\left[F\left(\underline{x}_{1}, \ldots, \underline{x}_{k}\right) \mid \underline{s}=s\right]
$$

is a non-decreasing function of $\mathrm{s}$.

\subsection{MOST POWERFUL TESTS AGAINST SIMPLE ALTERNATIVES}

Let $\underline{t}_{i}$ denote a test statistic for testing the hypothesis $\theta_{i}=\theta_{i}^{0}$ against the alternative $\theta_{i}>\theta_{i}^{0}, i=1,2, \ldots, k$, and let $\underline{t}_{1}, \underline{t}_{2}, \ldots, \underline{t}_{k}$ be independent $(k \gg 2)$. Suppose $t_{i}$ has density $p_{i}\left(t_{i} ; \theta_{i}\right)$ with respect to a $\sigma$-finite measure $\lambda_{i}$ defined on the Borel sets of $\mathbb{R}^{1}$ for all $\theta_{i} \in\left[\theta_{i}^{0}, \infty\right), i=1,2, \ldots, k$. We consider testing the simple hypothesis

$$
\mathrm{H}: \theta=\theta^{0}=\left(\theta_{1}^{0}, \ldots, \theta_{\mathrm{k}}^{0}\right)
$$

against the simple alternative 


$$
K^{\prime}: \theta=\theta^{\prime}=\left(\theta_{1}^{\prime}, \ldots, \theta_{k}^{\prime}\right), \theta^{\prime}: \geq \theta^{0}
$$

on the basis of $\underline{t}_{1}, \underline{t}_{2}, \ldots, \underline{t}_{k}$.

Let $L_{i}$ denote the logarithm of the likelihood ratio

$$
L_{i}\left(t_{i}\right)=\log p_{i}\left(t_{i} ; \theta !\right)-\log p_{i}\left(t_{i} ; \theta_{i}^{0}\right) \text {, }
$$

then, according to the NEYMAN-PEARSON lemma, the most powerful size- $\alpha$ test of $H$ against $K^{\prime}$ rejects $H$ if

$$
\text { (1.6.1) } \quad \sum_{i=1}^{k} L_{i}\left(t_{i}\right) \geq c,
$$

where $c$ depends on $\alpha$ (randomization may be necessary on the boundary of the critical region).

If the densities $p_{i}\left(t_{i} ; \theta_{i}\right)$ constitute one-parameter exponential families

$$
p_{i}\left(t_{i} ; \theta_{i}\right)=C_{i}\left(\theta_{i}\right) e^{Q_{i}\left(\theta_{i}\right) t_{i}}, \quad i=1,2, \ldots, k,
$$

where $Q_{i}$ is an increasing function, the most powerful size- $\alpha$ test of $\mathrm{H}$ against $\mathrm{K}^{\prime}$ rejects $\mathrm{H}$ if

$$
\sum_{i=1}^{k}\left\{Q_{i}\left(\theta_{i}^{\prime}\right)-Q_{i}\left(\theta_{i}^{0}\right)\right\} \underline{t}_{i} \geq c .
$$

Conversely, in the case of exponential family distributions any given linear combination procedure: reject $\mathrm{H}$ if

$$
\sum_{i=1}^{k} g_{i} \underline{t}_{i} \geq c \quad\left(g_{i} \geq 0, i=1,2, \ldots, k\right)
$$

is most powerful against all alternatives $\theta^{\prime}=\left(\theta_{1}^{\prime}, \ldots, \theta_{\mathrm{k}}^{\prime}\right)$ satisfying

$$
Q_{i}\left(\theta_{i}\right)-Q_{i}\left(\theta_{i}^{0}\right)=\gamma g_{i}, \quad \gamma>0, i=1,2, \ldots, k .
$$

Of course most powerful tests are only of practical value if the experimenter has more or less precise ideas about possible alternatives. But in that case a most powerful combination procedure should be used, because automatic application of some global combination method (e.g. FISHER's procedure) would involve a loss of power that may be considerable.

It is therefore of some interest to consider combination problems and investigate against which alternatives well-known combination procedures are most powerful. The combination problems to be considered are: (i) the combination 
of sign tests, (ii) the combination of tests in $2 \times 2$ tables and (iii) the combination of two-sample tests of WILCOXON. In a separate paragraph we pay some attention to FISHER's omnibus procedure (cf. section 1.2), since it is perhaps the best known combination method. The combination of t-tests is discussed in chapter 3 .

(i) Consider $\mathrm{k}$ independent binomial random variables $\underline{s}_{1}, \underline{s}_{2}, \ldots, \underline{s}_{k}$, where $\underline{s}_{i}$ is bin $\left(p_{i} ; n_{i}\right)$ distributed $(i=1,2, \ldots, k)$. The hypothesis to be tested is

$$
H: p=\left(p_{1}, \ldots, p_{k}\right)=\left(\frac{1}{2}, \ldots, \frac{1}{2}\right)
$$

against alternatives

$$
\mathrm{K}: \mathrm{p} . \geq\left(\frac{1}{2}, \ldots, \frac{1}{2}\right)
$$

This testing problem arises if $\mathrm{k}$ sign tests are to be combined. Put

$$
\theta_{i}=p_{i} /\left(1-p_{i}\right), \quad i=1,2, \ldots, k,
$$

then the distribution of $\underline{s}_{i}$ is an exponential family with respect to $\theta_{i}$, where $Q_{i}\left(\theta_{i}\right)=\log \theta_{i}(c f .(1 \cdot 6.2))$. Hence the most powerful combination procedure for testing $H$ against the simple alternative

$$
\mathrm{K}^{\prime}: \mathrm{p}=\mathrm{p}^{\prime} \cdot \geq\left(\frac{1}{2}, \ldots, \frac{1}{2}\right)
$$

rejects for large values of the test statistic

$$
\sum_{i=1}^{k} \underline{s}_{i} \log \theta_{i} \text {. }
$$

One possible way of testing $H$ against $K$ is based on pooling of all observations; $H$ is then rejected for large values of the sum statistic $\underline{S}=\sum \underline{s}_{i}$. It is easily seen that this sum test is most powerful against alternatives $\left(p_{1}^{\prime}, \ldots, p_{k}^{\prime}\right)$ satisfying $p_{1}^{\prime}=p_{2}^{\prime}=\ldots=p_{k}^{\prime}$. Application of this test is very simple, because $\underline{S}$ has a bin $\left(\frac{1}{2}, \sum_{i} n_{i}\right)$ distribution under $H$ and critical values are thus readily available.

If weights $g_{i}$ are introduced, i.e. if $H$ is rejected for large values of

$$
\underline{S}_{g}=\sum_{i=1}^{k} g_{i} s_{i} \quad\left(g_{i} \geq 0 \text { for } i=1,2, \ldots, k\right),
$$

this weighted sum test is most powerful against alternatives $\left(p_{1}^{\prime}, \ldots, p_{k}^{\prime}\right)$ 
satisfying

$$
p_{i}^{\prime} /\left(1-p_{i}^{\prime}\right)=e^{\gamma g_{i}} \quad, \gamma>0 \quad(i=1,2, \ldots, k) .
$$

To investigate this unwieldy relation more closely, we put

$$
p_{i}^{\prime}=\frac{1}{2}+\varepsilon_{i} \quad(i=1,2, \ldots, k)
$$

and obtain

$$
\log \left[p_{i}^{\prime} /\left(1-p_{i}^{\prime}\right)\right]=4 \varepsilon_{i}+\mathscr{O}\left(\varepsilon_{i}^{2}\right) \quad \text { for } \varepsilon_{i} \rightarrow 0
$$

Hence for small $\varepsilon_{i}$ ( $i . e$. for near alternatives) the test based on $\underline{S}_{g}$ will be approximately optimal against alternatives satisfying $\varepsilon_{i}=\gamma g_{i}(i=1,2, \ldots, k)$. Critical values of $\underline{S}_{g}$ may be obtained by direct computation (if the $n_{i}$ are very small and $\mathrm{k}$ is small) or by the normal approximation to $\underline{S}_{g}$ (if the $\mathrm{n}_{i}$ are not too small or $\mathrm{k}$ is rather large).

With the normal approximation in mind a test of $\mathrm{H}$ against $\mathrm{K}$ is often based on the sum of the standardized (under $H$ ) statistics $\underline{s}_{i}$. The resulting test is equivalent to a $\underline{S}_{g}$-test with $g_{i}=n_{i}^{-\frac{1}{2}}(i=1,2, \ldots, k)$. Hence the alternatives against which this test is most powerful heavily depend on the sample sizes $n_{i}$.

$$
\text { Consider } \mathrm{k} \text { 2×2 tables, } i=1,2, \ldots, \mathrm{k} \text { : }
$$

$\begin{array}{rccc} & \text { Success } & \text { Failure } & \text { Total } \\ \text { First series } & \underline{a}_{i} & \underline{c}_{i} & m_{i} \\ \text { Second series } & \underline{b}_{i} & \underline{d}_{i} & n_{i} \\ \text { Total } & \underline{r}_{i} & \underline{s}_{i} & m_{i}+n_{i}\end{array}$

The conditional test for testing equality of the probabilities $p_{i, 1}$ and $p_{i, 2}$ of success in the first and second series against the alternative $p_{i, 1}>p_{i, 2}$ rejects for large values of $\underline{a}_{i}$. Putting

$$
\theta_{i}=p_{i, 1}\left(1-p_{i, 2}\right) /\left\{p_{i, 2}\left(1-p_{i, 1}\right)\right\} \text {, }
$$

the conditional distribution of $\underline{a}_{i}$ (given $\underline{r}_{i}=r_{i}$ ) is an exponential family with respect to $\theta_{i}$, where $Q_{i}\left(\theta_{i}\right)=\log \theta_{i}(c f \cdot(1.6 .2))$. Hence the optimal combination procedure for testing

$$
\mathrm{H}: \theta=\left(\theta_{1}, \ldots, \theta_{\mathrm{k}}\right)=(1, \ldots, 1)
$$


against the simple alternative

$$
K^{\prime}: \theta=\theta^{\prime} \cdot \geq(1, \ldots, 1)
$$

rejects $\mathrm{H}$ for large values of

$$
\sum_{i=1}^{k} \underline{a}_{i} \log \theta_{i}
$$

However, in terms of $p_{i, 1}$ and $p_{i, 2}$ the alternative $K^{\prime}$ seems rather hard to interpret and it is therefore often preferred to test against alternatives like

$$
p_{i, 1}^{!}-p_{i, 2}^{\prime}=\varepsilon_{i}>0 \quad, i=1,2, \ldots, k
$$

For $\varepsilon_{i} \rightarrow 0$ we find

$$
\log \theta_{i}:=\varepsilon_{i} /\left\{p_{i, 1}\left(1-p_{i, 1}^{\prime}\right)\right\}+\sigma\left(\varepsilon_{i}^{2}\right)
$$

and thus $\log \theta_{i}^{\prime}$ depends on the unspecified value $p_{i, 1}^{\prime}$. But for large sample sizes $m_{i}$ and $n_{i}$ and $\operatorname{small} \varepsilon_{i}$ we may estimate $p_{i, 1}^{\prime}$ from the marginal totals and it turns out that if all $m_{i}$ and $n_{i}$ tend to infinity and $\varepsilon_{i}$ tends to zero as fast as $\left(m_{i}+n_{i}\right)^{-1}$, the conditional procedure that rejects $H$ for large values of

$$
\sum_{i=1}^{k} \frac{\left(m_{i}+n_{i}\right)^{2}}{r_{i} s_{i}} \varepsilon_{i} \underline{a}_{i}
$$

is asymptotically optimal in this case.

Similarly, the test that rejects $H$ for small values of the test statistic

$$
\sum_{i=1}^{k} \frac{m_{i}+n_{i}}{s_{i}} \varepsilon_{i}-_{i}
$$

is approximately optimal against alternatives of the form

$$
p_{i, 1}^{\prime} / p_{i, 2}^{\prime}=1+\varepsilon_{i} \quad, i=1,2, \ldots, k
$$

for large sample sizes $m_{i}$ and $n_{i}$ and $\operatorname{small} \varepsilon_{i}$.

Pooling of all data in a single $2 \times 2$ table leads to the test statistic $\sum_{i} \underline{a}_{i}$ conditional on $\sum_{i} \underline{r}_{i}=\sum_{i} r_{i}$. If $p_{1,1}=p_{2,1}=\ldots=p_{k, 1}$ and $p_{1,2}=p_{2,2}=\ldots$ $=p_{k, 2}$, this test statistic has a hypergeometric distribution under $\mathrm{H}$ and the related test is optimal. However, if the $p_{i, 1}$ and $p_{i, 2}$ do not have this property, the statistic has a complicated distribution both under $\mathrm{H}$ and the alternative and hence pooling is not indicated in this case. 
Conditional on $\underline{r}_{i}=r_{i}$ for $i=1,2, \ldots, k$ the test based on the statistic $\sum_{i} a_{i}$ is of course optimal against alternatives satisfying $\theta_{1}^{\prime}=\theta_{2}^{\prime}=\ldots=\theta_{k}^{\prime}$. The ${ }^{i}$ test statistic proposed by COCHRAN $[7]$ is in fact equivalent with $\sum_{i} a_{i}$; in his opinion situations where $\left(p_{i, 1}^{\prime}-p_{i, 2}^{\prime}\right) /\left\{p_{i, 1}^{\prime}\left(1-p_{i, 1}^{\prime}\right)\right\}$ is constant $i_{\text {frequently }}$ occur in applications and the test based on $\sum \underline{a}_{i}$ is approximately optimal in this case (cf. $(1.6 .8)$ ). Weighted versions of this test have been proposed by several authors for testing $H$ against $K$. If the $\underline{a}_{i}$ are standardized (under $H$, conditional on $\left.\underline{r}_{i}=r_{i}\right)$ and added, the weights attached to each $\underline{a}_{i}$ are $g_{i}=\left(m_{i}+n_{i}\right)\left(m_{i}+n_{i}-1\right)^{\frac{1}{2}}$. $\left(m_{i} n_{i} r_{i} s_{i}\right)^{-\frac{1}{2}}, i=1,2, \ldots, k$. In C.L. RUMKE and C. VAN EEDEN [42] the weights $g_{i}=\left(m_{i}+n_{i}\right) / m_{i} n_{i}$ are advised. In both cases the alternatives against which these weighted versions of the $\sum_{i} a_{i}$ test are optimal depend on the sample sizes in a rather intricate way.

To determine critical values of all the above combination methods one has to rely on the asymptotic normality of the test statistics, unless the sample sizes are so small that exact computation is feasible.

(iii) Consider $k$ pairs of samples of independent observations $x_{i, 1}, x_{i, 2}, \ldots$ , $\underline{x}_{i, m_{i}}$ and $\underline{y}_{i, 1}, \underline{y}_{i, 2}, \ldots, \underline{y}_{i, n_{j}}, i=1,2, \ldots, k$, where $\underline{x}_{i, j}$ and $y_{i, j}$ have continuous distribution functions $F_{i}$ and $G_{i}$ respectively and $G_{i}(x)=F_{i}\left(x_{i}-\Delta_{i}\right)$. Let $U_{i}$ denote the number of pairs $\left(\underline{x}_{i, j}, \underline{y}_{i, j}\right)$ in the $i$-th sample with $\underline{x}_{i, j}<\underline{y}_{i, j}$, , $j=1,2, \ldots, m_{i}, j^{\prime}=1,2, \ldots, n_{i}$.Then WILCOXON's two-sample test for testing $\Delta_{i}=0$ against $\Delta_{i}>0$ rejects for large values of the statistic $\underline{U}_{i}$. The hypothesis to be tested in this case is

$$
\mathrm{H}: \Delta=\left(\Delta_{1}, \ldots, \Delta_{k}\right)=0
$$

against

$$
\mathrm{K}: \Delta \cdot \mathbf{0}
$$

Since this is a nonparametric problem, only asymptotical results can be obtained. We suppose that $m_{i}$ and $n_{i}$ tend to infinity in such a way that $m_{i} / n_{i}$ tends to a positive finite limit and that the true value $\Delta_{i}$ of $\Delta_{i}$ tends to zero. Putting

$$
\theta_{i}=\int F_{i}(x) d F_{i}\left(x_{i}-\Delta_{i}\right),
$$

it can be shown (cf. [16] and [60]) that the test that rejects $H$ for large values 
of the statistic

$$
\sum_{i=1}^{k}\left(\theta !-\frac{1}{2}\right)\left(m_{i}+n_{i}+1\right)^{-1} \underline{U}_{i}
$$

is an asymptotically most powerful test of $\mathrm{H}$ against near alternatives $\Delta_{i}=\Delta_{i}(i=1,2, \ldots, k)$. In case $\theta_{1}^{\prime}=\theta_{2}^{\prime}=\ldots=\theta_{k}^{\prime}$, the test statistic $(1.6 .11)$ reduces to

$$
\sum_{i=1}^{k}\left(m_{i}+n_{i}+1\right)^{-1} \underline{U}_{i} .
$$

This test was first proposed by VAN ELTEREN [16] who calls it the locally best $\mathrm{W}$-test. He also put forward another test based on the statistic

$$
\sum_{i=1}^{k}\left(m_{i} n_{i}\right)^{-1} \underline{U}_{i},
$$

which is obviously also asymptotically most powerful against appropriate alternatives depending on the sample sizes. This combination procedure is also advised in [42]. VAN ELTEREN proposed the test (1.6.12) because it is a designfree test, i.e. its consistency does not depend on the sample sizes. The designfree approach is motivated by the fact that it is desirable to reject $H$ if the majority of the $\Delta_{i}$ are positive and only a few are negative. However, this argument does not apply in the present context, since we have assumed the $\Delta_{i}$ to be nonnegative.

If the $\underline{U}_{i}$ are standardized (under $H$ ) and added, the resulting linear combination of the $\underline{U}_{i}$ is an asymptotically optimal test statistic against near alternatives $\theta^{\prime}$ satisfying $\theta_{i}^{\prime}-\frac{1}{2}=\gamma\left(m_{i}+n_{i}+1\right)^{\frac{1}{2}} m_{i}^{-\frac{1}{2}} n_{i}^{-\frac{1}{2}}(\gamma>0, i=1,2, \ldots, k)$.

Critical values of a linear combination procedure may again be determined by the normal approximation to the test statistic.

In each of the examples (i), (ii) and (iii) it is seen that the linear combination obtained by adding the standardized individual test statistics provides a test of $\mathrm{H}$ which is (asymptotically) optimal against alternatives $\theta^{\prime}$ depending strongly on the sample sizes, relatively large sample sizes corresponding to relatively small $\theta_{i}$. Nevertheless we shall find in the sequel that in the large sample case these tests enjoy certain optimum properties among all linear combination procedures if all possible alternatives are taken into account.

In some applications the sampling design is such that the sample sizes $\mathrm{n}_{i}$ of the individual experiments are proportional to the respective population sizes $N_{i}(i=1,2, \ldots, k)$. The combination problem is not essentially influenced by such a 
design. It may also happen that the hypotheses are formulated in terms of the $N_{i}$. This occurs e.g. if the hypothesis $\sum_{N_{i}} \theta_{i}=0$ is to be tested against the alternative $\left[\mathrm{N}_{i} \theta_{i}>0\right.$. If the $\theta_{i}$ are known to be nonnegative, this problem reduces to the original one-sided combination problem of testing $\theta=0$ against $\theta . \geq 0$.

If on the other hand the $\theta_{i}$ are not restricted to be nonnegative, we get an entirely different testing problem outside the scope of this study.

Finally we make a few remarks on FISHER's omnibus combination procedure. We assume that $\underline{t}_{1}, \underline{t}_{2}, \ldots, \underline{t}_{k}$ are distributed according to the exponential families (1.6.2) and that the hypothesis $H: \theta=\theta^{0}$ is to be tested against the alternative $K: \theta . \geq \theta^{0}$. In this case FISHER's procedure rejects $H$ for small values of the statistic

$$
\prod_{i=1}^{k}\left[1-F_{i}\left(\underline{t}_{i} ; \theta_{i}^{0}\right)\right],
$$

where $F_{i}\left(. ; \theta_{i}^{0}\right)$ denotes the distribution function of $t_{i}$ if $\theta_{i}=\theta_{i}^{0}(i=1,2, \ldots, k)$. It is rather exceptional that the test statistic (1.6.13) is equivalent to a linear function of $\underline{t}_{1}, \underline{t}_{2}, \ldots, \underline{t}_{k}$ and hence FISHER's procedure will in general not be most powerful against a simple alternative. Moreover, it seems to be unknown whether FISHER's test is admissible for all one-sided combination problems in exponential families. Since the tests with a.e. convex and monotone acceptance regions constitute an essentially complete class (cf.section 1.4), it would be a first prerequisite to show that FISHER's test belongs to this class. The monotonicity is evident, but to the author's knowledge the convexity of the acceptance region has been established only in special cases.

Properties of FISHER's omnibus procedure have usually been studied starting from the one-sided tail probabilities $\underline{p}_{i}=1-F_{i}\left(\underline{t}_{i} ; \theta_{i}\right), i=1,2, \ldots, k$. It is then assumed(in the absolutely continuous case) that the hypothesis

$$
\mathrm{H}^{*}: \mathrm{p}_{\mathrm{i}} \text { is uniformly distributed on }[0,1], \mathrm{i}=1,2, \ldots, \mathrm{k}
$$

is to be tested against the alternative

$$
\mathrm{K}^{*}: \mathrm{p}_{\mathrm{i}} \text { has a non-decreasing density on }[0,1] \text {, }
$$
$i=1,2, \ldots, k$. It has first been shown by A. BIRNBAUM [ 4] (cf.also T. LIPTAK [34]) that any test with a monotone acceptance region in $\left(p_{1}, \ldots, p_{k}\right)$-space is most powerful against a simple alternative in $K^{*}$. This is not surprising, because the class of alternative distributions defined by $\mathrm{K}^{*}$ is a very large class indeed. 
Since FISHER's procedure has a monotone acceptance region in $\left(p_{1}, \ldots p_{k}\right)$-space, it is most powerful against a simple alternative in $K^{*}$. In fact, it has already been shown in 1938 by E.S. PEARSON [36] that the omnibus test is most powerful against the alternative that each $p_{i}$ is distributed with density $(m+1)\left(1-p_{i}\right)^{m}$, $-1<\mathrm{m}<0$. However, these results are only of a limited value, since in most applications the class of alternative distributions will be much smaller than $K^{*}$. 
CHAPTER 2. NORMALLY DISTRIBUTED STATISTICS WITH KNNOWN VARIANCES

In this chapter, which is based on a joint paper [60] by W.R. VAN ZWET and the author, procedures for combining $\mathrm{k}$ normal random variables with known variances are discussed.

In section 2.1 it is shown that many combination problems asymptotically reduce to this problem for large sample sizes. In section 2.2 some fundamental properties of admissible combination procedures are established and in section 2.3 the most stringent (MS) test is constructed in a number of special cases. Some of the results are not only derived for the normal case, but more generally for one-parameter exponential families of distributions satisfying appropriate conditions. In section 2.4 it is shown that a class of tests exists, including the likelihood ratio (LR) test and FISHER's omnibus procedure, having uniformly vanishing shortcoming as the size $\alpha$ tends to zero. An asymptotic expression for the shortcoming of the LR test is derived and the asymptotic shortcoming of several combination methods is studied in some detail (for $\alpha \rightarrow 0$ ). Finally in section 2.5 various combination procedures are compared numerically. It turns out that for moderate or small sizes the LR test and FISHER's procedure are rather satisfactory if no prior information concerning the $\mathrm{k}$ means is available. However, when there is some prior information indicating that the means of the normal distributions are not very much different, symmetric linear combination is more adequate.

2.1. LARGE SAMPLE THEORY

The combination problem to be investigated may be formulated in the following way. For $i=1,2, \ldots, k$ let $\underline{t}_{i} n_{i}$ denote $k$ independent test statistics, based on $n_{i}$ observations, for testing ${ }^{i}$ the hypothesis $H_{i, 0}: \theta_{i}=\theta_{i}^{0}$ against alternatives $\theta_{i}>\theta_{i}^{0}$. We wish to test the combined hypothesis 


$$
H^{*}: \theta=\theta^{0}
$$

against one-gided alternatives

$$
\mathrm{K}^{*}: \theta \cdot \geq \theta^{0},
$$

where $\theta$ denotes the vector $\left(\theta_{1}, \ldots, \theta_{k}\right)$.

For most combination problems UMP, UMP unbiased or UMP invariant tests of $\mathrm{H}^{*}$ against $\mathrm{K}^{*}$ do not exist. Lacking other satisfactory criteria, one may then try to find a minimax test with respect to some risk function, or any other test which closely resembles such a minimax test. Our interest will be concentrated on MS tests, because they are uniquely determined, as opposed to maximin tests which depend on the zone of indifference (cf.section 1.3).

In most applications the power functions of the $\mathrm{k}$ individual test statistics $t_{i, n}$ are sufficiently intractable to defeat any attempt to obtain satisfactory comblnation procedures for testing $\mathrm{H}^{*}$ against $\mathrm{K}^{*}$ in the small sample case. However, the statistics $\underline{t}_{i, n}$ are often asymptotically normally distributed for increasing sample sizes, not only under $\mathrm{H}_{i, 0}$ but also for sequences of alternatives tending sufficiently rapid to the null hypothesis as $n_{i}+\infty$. Exploiting this property we may try to find combination methods which are at least asymptotically optimal in some sense. This is the classical approach to complicated testing problems which has first been applied by J. NEYMAN and E.J.G. PITMAN. In chapter 4 we shall briefly discuss a different asymptotic approach where the size $\alpha$ is a function of the sample sizes and tends to zero as $n_{i} \rightarrow \infty$. But for the present we assume that the significance level $\alpha$ is fixed.

Let the sample sizes $n_{i}$ be functions of an integer $N$ such that $\lim _{N \rightarrow \infty} n_{i}(N)=\infty$ for $i=1,2, \ldots, k$. Then we may write $t_{i, N}$ instead of $t_{i, n}$. Our asymptotic approach is based on the following result of $\mathrm{P}$. BILLINGSLEY and ${ }_{\mathrm{F}}$.TOPS $\emptyset \mathrm{E}$ [3 ].

Lemma 2.1.1 (P. BILLINGSLEY and F. TOPS $\varnothing \mathrm{E}$ )

Let $\left\{\mathrm{F}_{\mathbb{N}}\right\}$ denote a sequence of 'distribution functions defined on $\mathbb{R}^{\mathrm{k}}(\mathrm{k} \geq 2)$ converging weakly. to a distribution function $F\left(i \cdot e \cdot F_{N}\right.$ converges pointwise to $F$ on the set of all continuity points of $F$ ). Let $\mathcal{S}$ denote a class of Borel sets $A$ of $\mathbb{R}^{\mathrm{k}}$ satisfying

$$
\lim _{\varepsilon \rightarrow 0} \sup _{A \in \mathcal{S}} P(U(\partial A, \varepsilon))=0
$$

where $P$ is the probability measure induced by $F$ and $U(\partial A, \varepsilon)$ is the $\varepsilon$-neighborhood 
of the boundary of A. Then

$(2.1 .2)$

$$
\lim _{\mathbb{N} \rightarrow \infty} \int_{A} d F_{N}=\int_{A} d F \quad \text { uniformly for all } A \in \mathcal{S} .
$$

With the aid of this lemma we prove

\section{Theorem 2.1.1}

Let $\left\{\mathrm{F}_{\mathrm{N}}\right\}$ denote a sequence of distribution functions defined on $\mathbb{R}^{\mathrm{k}}(\mathrm{k}>2)$ converging weakly to an absolutely continuous distribution function $F$. Let $\overline{\tilde{\mathcal{M}}}$ denote the class of all monotone Borel sets of $\mathbb{R}^{\mathrm{k}}$ (cf.section 1.4).

Then

$$
\lim _{\mathbb{N} \rightarrow \infty} \int_{A} d F_{N}=\int_{A} d F \text {, uniformly for all } A \in \tilde{\mathcal{M}} \text {. }
$$

Proof:

Let $\delta>0$ be arbitrary and let $\lambda=\lambda_{1} \times \ldots \times \lambda_{k}$ denote Lebesgue measure on $\mathbb{R}^{k}$. Since $F$ is absolutely continuous, there exists $\varepsilon>0$ such that for any Borel set $B \subset \mathbb{R}^{k}$

$$
\lambda(B)<\varepsilon \text { implies } P(B)<\frac{1}{2} \delta,
$$

where $\mathrm{P}$ denotes the probability measure induced by $\mathrm{F}$. Perform a rotation of the coordinate axes $x_{1}, x_{2}, \ldots, x_{k}$ in $\mathbb{R}^{k}$ to new axes $x_{1}^{\prime}, x_{2}^{\prime}, \ldots, x_{k}^{\prime}$ such that the new $x_{1}^{\prime}$ - axis coincides with the line $x_{1}=x_{2}=\ldots=x_{k}$. Let $D$ be the set $\left\{x^{\prime} \mid-d \leq x_{i}^{\prime} \leq d\right.$, $i=1,2, \ldots, k\}$ and choose $d$ so large that $P(D)>1-\frac{1}{2} \delta$. Let $0<\varepsilon_{1}<\varepsilon\left\{2 k^{\frac{1}{2}}(2 d)^{k-1}\right\}^{\frac{1}{2}-1}$, let $A$ be an arbitrary monotone set in $\mathbb{R}^{k}$ and $U\left(\partial A, \varepsilon_{1}\right)$ the $\varepsilon_{y}$-neighborhood of its boundary. It follows from the monotonicity of the set $A$ that any line parallel to the $x_{1}^{\prime}$-axis has at most a segment of length $2 \varepsilon_{\uparrow} k^{\frac{1}{2}}$ in common with $U\left(\partial A, \varepsilon_{1}\right)$. Hence $\lambda\left(U\left(\partial A, \varepsilon_{1}\right) \cap D\right)=\int_{-d}^{d} \ldots \int_{-d}^{d} \lambda_{1}\left(\left\{x_{1}^{\prime} \mid x^{\prime} \in U\left(\partial A, \varepsilon_{1}\right) \cap D\right\}\right) d x_{2}^{\prime} \ldots d x_{k}^{\prime} \leq 2 \varepsilon_{1} k^{\frac{1}{2}}(2 d)^{k-1}<\varepsilon$, and therefore, $\mathrm{by}(2.1 .4), \mathrm{P}\left(\mathrm{U}\left(\partial \mathrm{A}, \varepsilon_{1}\right) \cap \mathrm{D}\right)<\frac{1}{2} \delta$. Thus

$$
\mathrm{P}\left(\mathrm{U}\left(\partial \mathrm{A}, \varepsilon_{1}\right)\right) \leq \mathrm{P}\left(\mathrm{U}\left(\partial \mathrm{A}, \varepsilon_{1}\right) \cap \mathrm{D}\right)+1-\mathrm{P}(\mathrm{D})<\delta .
$$

As $\delta$ was arbitrary and $\varepsilon_{1}$ does not depend on the particular set $A \in \tilde{\mathcal{M}}$, it follows that

$$
\lim _{\varepsilon_{1} \rightarrow 0} \sup _{A \in \tilde{\mathcal{M}}} P\left(U\left(\partial A, \varepsilon_{1}\right)\right)=0
$$

and application of lemma 2.1.1 completes the proof of the theorem. 


\section{Corollary 2.1.1}

Let $\underline{z}_{1}, \underline{z}_{2}, \ldots, \underline{z}_{k}$ be independent and normally distributed random variables with means $\mu_{1}, \mu_{2}, \ldots, \mu_{k}$ and unit variances. Let the sequence of random vectors $\underline{\mathrm{z}}^{(\mathbb{N})}=\left(\underline{\mathrm{z}}_{1, \mathrm{~N}}, \ldots, \underline{\mathrm{z}}_{\mathrm{k}, \mathrm{N}}\right)$ for $\mathrm{N} \rightarrow \infty$ converge in law to the distribution of $\underline{\mathrm{z}}=$ $\left(\underline{z}_{1}, \ldots, \underline{z}_{k}\right)$. Then

$$
\lim _{\mathbb{N} \rightarrow \infty} P\left(\psi\left(\underline{z}^{(N)}\right) \leq c\right)=P(\psi(\underline{z}) \leq c)
$$

uniformly for all real $c$ and all functions $\psi$ which are non-decreasing in the vector $\mathrm{z}$ (cf.section 1.4).

Now assume that the independent test statistics $t_{i, N}$ are asymptotically normal, $i . e$. there exist positive numbers $\sigma_{i, N}$ and real-valued functions $\mu_{i, N}$ such that, if $\theta_{i, N}$ are the true values of the parameters $\theta_{i}$,

$$
\left(\underline{t}_{i, N}-\mu_{i, N}\left(\theta_{i, N}\right)\right) / \sigma_{i, N} \quad, i=1,2, \ldots, k,
$$

tend in law to the standard normal distribution for $\mathbb{N} \rightarrow \infty$ for any sequence $\left\{\theta_{i, N}\right\}$ satisfying

$$
\lim _{N \rightarrow \infty} \theta_{i, N}=\theta_{i}^{0}, \quad i=1,2, \ldots, k
$$

Given the statistics $t_{1, N}, t_{2, N}, \cdots, t_{k, N}$ we wish to test the hypothesis $H^{*}$ against alternatives

$$
\mathrm{K}_{\mathrm{N}}^{*}: \theta_{i}=\theta_{i, N} \quad, i=1,2, \ldots, k,
$$

satisfying the conditions

$$
\lim _{N \rightarrow \infty} \theta_{i, N}=\theta_{i}^{0},
$$

$$
\lim _{N \rightarrow \infty}\left\{\mu_{i, N}\left(\theta_{i, N}\right)-\mu_{i, N}\left(\theta_{i}^{0}\right)\right\} / \sigma_{i, N}=\mu_{i}(\text { say }) \geq 0
$$

for $i=1,2, \ldots, k$, with $\mu_{i}>0$ at least once.

Define

$$
\underline{z}_{i, N}=\left(\underline{t}_{i, N}-\mu_{i, N}\left(\theta_{i}^{0}\right)\right) / \sigma_{i, N} \quad, \quad i=1,2, \ldots, k .
$$

Then obviously $\underline{z}_{i, N}$ is asymptotically $N(0,1)$ under $H^{*}$ and asymptotically $N\left(\mu_{i}, 1\right)$ under the sequence of alternatives $\mathrm{K}_{\mathrm{N}}^{*}$. 
Consider any monotone combination procedure of limiting size $\alpha$, i.e. a procedure

$$
\text { reject } H^{*} \text { if } \psi\left(\underline{z}_{1, N}, \ldots, \underline{z}_{k, N}\right) \geq c \text {, }
$$

where $\psi$ is non-decreasing in its vector argument and

$$
\lim _{\mathbb{N} \rightarrow \infty} \alpha_{N}=\lim _{\mathbb{N} \rightarrow \infty} P\left(\psi\left(\underline{z}_{1, N}, \ldots, \underline{z}_{k, N}\right) \geq c \mid H^{*}\right)=\alpha .
$$

Let $\underline{x}_{1}, \underline{x}_{2}, \ldots, \underline{x}_{k}$ be independent and let $\underline{x}_{i}$ be $N\left(\mu_{i}, 1\right), i=1,2, \ldots, k$. Consider the hypothesis

$$
\mathrm{H}: \mu=\left(\mu_{1}, \ldots, \mu_{k}\right)=0
$$

and the composite one-sided alternative

$$
\mathrm{K}: \mu \cdot \geq 0 \text {. }
$$

Then according to corollary 2.1.1, the limiting power for $\mathbb{N} \rightarrow \infty$ of the monotone combination procedure $(2.1 .9)$ against $K_{N}^{*}$ is equal to the power of the monotone size- $\alpha$ procedure

$$
\text { reject } H \text { if } \psi\left(\underline{x}_{1}, \ldots, \underline{x}_{k}\right) \geq c
$$

for testing $H$ against the alternative $\mu$ defined by (2.1.8).

Suppose we adopt some optimality criterion based on the power of the combination procedure and find an optimal test of $\mathrm{H}$ against $\mathrm{K}$. If this test is monotone, the procedure obtained by replacing $\underline{x}_{i}$ by $\underline{z}_{i, N}$ is asymptotically optimal for testing $\mathrm{H}^{*}$ against all alternatives of the form $\mathrm{K}_{\mathrm{N}}^{*}$ among all monotone combination procedures of limiting size a for this problem. If the optimality criterion adopted is stringency, this conclusion remains true provided the most powerful tests of $\mathrm{H}^{*}$ against simple alternatives are also monotone.

In the subsequent sections of this chapter the problem of testing $H$ against $\mathrm{K}$ will be discussed. Optimal tests of $\mathrm{H}$ against $\mathrm{K}$ are necessarily monotone since all other tests are inadmissible (lemma 1.4.6). Moreover, we have shown (theorem 1.4.3) that monotone procedures of size a constitute an essentially complete class for testing $\mathrm{H}^{*}$ against $\mathrm{K}^{*}$ whenever the densities of the individual test statistics $t_{i, N}$ have strict MLR in $t_{i, N}$. Hence the construction of an optimal test of $H$ against $\mathrm{K}$ yields an asymptotically optimal test of $\mathrm{H}^{*}$ against $\mathrm{K}^{*}$ in this case. 
Of course the monotonicity condition is unnecessarily restrictive and the limit relation (2.1.5), on which the preceding discussion was based, holds for a much larger class of test statistics $\psi$. Hence we may expect that our argument remains valid in many cases where the densities of the individual test statistics do not have strict $M L R$ in $t_{i, N}$. In the author's opinion the assertion of corollary 2.1.1 will break down only in pathological situations, where the individual statistics $\underline{t}_{i, N}$ are inadequate for testing $H_{i, 0}$. Obviously, the combination of poor tests will only give rise to poor combination procedures.

We conclude this section with two simple examples.

\section{Example 2.1.1}

Let $\mathrm{y}_{i, 1}, \mathrm{y}_{i, 2}, \ldots, \mathrm{y}_{i, n_{i}}, i=1,2, \ldots, k$, be $\mathrm{k}$ samples of independent normal $\mathrm{N}\left(v_{i}, \sigma_{i}^{2}\right)$ observations with unknown $v_{i}$ and $\sigma_{i}^{2}$. To test the hypothesis $\mathrm{H}_{i, 0}: v_{i}=0$ against $v_{i}>0$ the optimal (UMP unbiased and UMP invariant) size- $\alpha$ test is the STUDENT t-test

where

$$
\text { reject } H_{i, 0} \text { if } \underline{t}_{i, n_{i}} \geq c_{\alpha, n_{i}} \text {, }
$$

$$
\underline{t}_{i, n_{i}}=y_{i .} n_{i}^{\frac{1}{2}} / \underline{s}_{i} \quad, z_{i}=\frac{1}{n_{i}} \sum_{j=1}^{n_{i}} y_{i j}, s_{i}^{2}=\frac{1}{n_{i}-1} \sum_{j=1}^{n_{i}}\left(y_{i j}-y_{i}\right)^{2}
$$

and $c_{\alpha, n_{i}}$ is an appropriate critical value.

Let $v_{i, n_{i}}$ denote the true value of $v_{i}(i=1,2, \ldots, k)$. Writing

$$
t_{i, n_{i}}=\left(y_{i}-v_{i, n_{i}}\right) n_{i}^{\frac{1}{2}} / \underline{s}_{i}+v_{i, n_{i}} n_{i}^{\frac{1}{2}} / s_{i}, i=1,2, \ldots, k \text {, }
$$

the first term of the right hand member is asymptotically $\mathbb{N}(0,1)$ for $n_{i} \rightarrow \infty$ and the second term converges in probability to

$$
\mu_{i}=\lim _{n_{i} \rightarrow \infty} v_{i, n_{i}} n_{i}^{\frac{1}{2}} / \sigma_{i} \quad, i=1,2, \ldots, k,
$$

provided the limit exists. Hence $t_{i, n}$ tends in law to the $N\left(\mu_{i}, 1\right)$ distribution if $v_{i, n}$ is of order $n_{i}^{-\frac{1}{2}}(i=1,2, \ldots, k)^{i}$ Moreover, the non-central t-distribution has strict MLR in $t$ (cf. LEHMANN [31]p.233). Therefore the asymptotic theory of this section can be applied to test the hypothesis $H^{*}: \nu=\left(\nu_{1}, \ldots, \nu_{k}\right)=0$ against $\mathrm{K}^{*}: \nu \cdot \geq 0$. 
If the variances $\sigma_{i}^{2}$ are known to be equal to some unknown $\sigma^{2}$,

$$
\underline{s}^{2}=\sum_{i=1}^{k}\left(n_{i}-1\right) \underline{s}_{i}^{2} / \sum_{j=1}^{k}\left(n_{j}-1\right)
$$

is the best estimator of $\sigma^{2}$ and it is intuitively appealing to base tests of $H^{*}$ against $K^{*}$ on the statistics $t_{i, n}^{*}=y_{i} . n_{i}^{\frac{1}{2}} / s, i=1,2, \ldots, k$. These statistics are not independent, but their joint distribution converges to the joint distribution of $\mathrm{k}$ independent $\mathrm{N}\left(\mu_{i}, 1\right)$ random variables under the same condition as in the preceding case. Hence corollary 2.1.1 is again applicable. More details are given in chapter 3 .

\section{Example 2.1.2}

Let $\underline{s}_{1, n_{1}}, \underline{s}_{2, n_{2}}, \ldots, s_{k, n_{k}}$ denote the number of successes in $k$ series of Bernoulli trials. In the $i-$ th series $n_{i}$ independent trials are performed, each with probability $p_{i}$ of succes, $i=1,2, \ldots, k$. We wisk to test the combined hypothesis $H^{*}: p_{1}=p_{2}=\ldots=p_{k}=\frac{1}{2}$ against $K^{*}: p_{i} \geq \frac{1}{2}$ for all $i$ with strict inequality at least once. This problem arises if $\mathrm{k}$ sign tests are to be combined (cf.section 1.6 (i)). The UMP test of $\mathrm{H}_{i, 0}: p_{i}=\frac{1}{2}$ against $p_{i}>\frac{1}{2}$ is of course the one-sided binomial test based on $\underline{s}_{i, n_{i}}$, or equivalently on

$$
\underline{t}_{i, n_{i}}=2 n_{i}^{-\frac{1}{2}}\left(\underline{s}_{i, n_{i}}-\frac{1}{2} n_{i}\right) \quad, i=1,2, \ldots, k \text {. }
$$

If $p_{i, n_{i}}$ is the true value of $p_{i}$ and

$$
\mu_{i}=2 \lim _{n \rightarrow+\infty}\left(p_{i, n_{i}}-\frac{1}{2}\right) n_{i}^{\frac{1}{2}} \quad, i=1,2, \ldots, k,
$$

exists, i.e. if $p_{i, n_{i}}-\frac{1}{2}$ is of order $n_{i}^{-\frac{1}{2}}$, then it is again easily verified that $\underline{t}_{i, n_{i}}$ tends in $l_{\text {aw }}$ to the $N\left(\mu_{i}, 1\right)$ distribution. As the distribution of $t_{i, n_{i}}$ has strict MLR in $t_{i, n_{i}}$, optimal tests of $H$ against $K$ in the normal case lead to asymptotically optimal tests for this problem also.

We note that in the exceptional case where $n_{1}=n_{2}=\ldots=n_{k}=1$, an UMP invariant test of $\mathrm{H}^{*}$ against $\mathrm{K}^{*}$ exists, viz. the sign test applied to $\underline{\mathrm{s}}_{1,1}, \underline{\mathrm{s}}_{2,1}, \ldots, \underline{\mathrm{s}}_{\mathrm{k}, 1}$ (cf. LEHMANN [31]p.219). 


\subsection{GENERAL PROPERTIES}

In the preceding section it was shown that for sample sizes tending to infinity many combination problems asymptotically reduce to the following testing problem.

Let $\underline{x}_{1}, \underline{x}_{2}, \ldots, \underline{x}_{k}$ denote $k$ independent random variables $(k \geq 2)$, where $\underline{x}_{i}$ has a normal $N\left(\mu_{i}, 1\right)$ distribution, $i=1,2, \ldots, k$. The hypothesis

$$
\mathrm{H}: \mu=\left(\mu_{1}, \ldots, \mu_{\mathrm{k}}\right)=0
$$

is to be tested at level $\alpha$ against the one-sided alternative

$$
\mathrm{K}: \mu \cdot \geq 0 \text {. }
$$

First let us assume that the alternative $\mu$ is known. Application of (1.6.3) yields that the most powerful size- $\alpha$ test of $\mathrm{H}$ against the simple alternative $\mu$ rejects $\mathrm{H}$ if

$$
\sum_{i=1}^{k} \mu_{i} \underline{x}_{i} \geq u_{\alpha}\left(\sum_{i=1}^{k} \mu_{i}^{2}\right)^{\frac{1}{2}},
$$

where $u_{\alpha}$ denotes the upper $\alpha$-point of the standard normal distribution. The power of the test against this alternative is easily seen to be equal to

$$
\beta_{\alpha}^{+}\left(\mu_{1}, \ldots, \mu_{k}\right)=1-\Phi\left(u_{\alpha}-\left(\sum_{i=1}^{k} \mu_{i}^{2}\right)^{\frac{1}{2}}\right),
$$

where $\Phi$ denotes the standard normal distribution function.

If the alternative is unknown and $H$ is tested against $K,(2.2 .2)$ denotes the size- $\alpha$ envelope power function.

In our search for tests with uniformly good power properties for all alternatives in $\mathrm{K}$ we may as well restrict our attention to nonrandomized tests with monotone and convex acceptance regions (cf. section 1.4), since according to lemma 1.4.6 these tests constitute a minimal essentially complete class.

We note that the monotonicity of the admissible procedures ensures that any admissible size- $\alpha$ test of $H$ against $K$ is also an admissible size- $\alpha$ test of the extended null hypothesis

$$
\mathrm{H}_{\text {ext }}: \mu \leq 0
$$

against K. Similarly, any optimal size- $\alpha$ test of $H$ against $K$ is also optimal 
for testing $\mathrm{H}_{\text {ext }}$ against $\mathrm{K}$.

The class of admissible size- $\alpha$ tests is essentially larger than the class of all size- $\alpha$ Bayes tests, as is demonstrated by the following theorem.

\section{Theorem 2.2.1}

For any $k \geq 2$ let $A$ denote the acceptance region of a size- $\alpha$ Bayes test of $H$ against $K$. Then any line of support of $A$ either meets clos(A) at exactly one point or it is contained in the boundary of $A$.

Proof:

Let $G(\mu)$ denote an arbitrary prior distribution function on the set $\left\{\mu \mid \mu=\left(\mu_{1}, \ldots, \mu_{k}\right) . \geq 0\right\}$. The size- $\alpha$ Bayes test of $\mathrm{H}$ against this prior distributicion rejects $\mathrm{H}$ if

$$
t(\underline{x})=t\left(\underline{x}_{1}, \ldots, \underline{x}_{k}\right) d \underline{\underline{e} f} \int_{0}^{\infty} \ldots \int_{0}^{\infty} \exp \left(\sum_{i=1}^{k} \mu_{i} \underline{x}_{i}\right) d G^{*}(\mu) \geq c,
$$

where

$$
\mathrm{dG}^{*}(\mu)=\exp \left(-\frac{1}{2} \sum_{i=1}^{\mathrm{k}} \mu_{i}^{2}\right) \mathrm{dG}(\mu)
$$

and $c$ is an appropriate constant. Let $u=\left(u_{1}, \ldots, u_{k}\right)$ and $v=\left(v_{1}, \ldots, v_{k}\right)$ be two points on the boundary of the acceptance region $A$, i.e.

$$
t(u)=t(v)=c,
$$

and let $\mathrm{P}^{*}$ denote the measure induced by $G^{*}$ on the parameter space. We consider two cases.

a) Suppose $P^{*}\left(\sum_{i=1}^{k} \mu_{i}\left(u_{i}-v_{i}\right) \neq 0\right)=0$. Let $z=\left(z_{1}, \ldots, z_{k}\right)$ be defined by $z=\rho u+(1-\rho) v i=1,-\infty<\rho<\infty$. Then

$$
t(z)=\int_{0}^{\infty} \ldots \int_{0}^{\infty} \exp \left[\sum_{i=1}^{k} \mu_{i} v_{i}+p \sum_{i=1}^{k} \mu_{i}\left(u_{i}-v_{i}\right)\right] d G^{*}(\mu)=c .
$$

Hence the line through $u$ and $v$ is also part of the boundary of $A$ in this case.

b) Suppose $P^{*}\left(\sum_{i=1}^{k} \mu_{i}\left(u_{i}-v_{i}\right) \neq 0\right)>0$, and let $0<\rho<1$. Since

$$
\exp (\rho g+(1-\rho) h) \leq \rho e^{g}+(1-\rho) e^{h}
$$

for all real $g$ and $h$ with equality if and only if $g=h$, we find that for any point $z$ defined by $z=\rho, u+(1-\beta) v$

$$
t(z)<\rho t(u)+(1-\rho) t(v)=c .
$$


This implies that the line through $u$ and $v$ is not a line of support of $A$ since any point $\mathrm{z}$ between $\mathrm{u}$ and $\mathrm{v}$ is an interior point of $\mathrm{A}$.

It would be of some interest to characterize the boundaries of acceptance regions of Bayes tests, but unfortunately simple explicit conditions seem difficult to obtain.

The validity of theorem 2.2.1 is not restricted to the case where $\underline{x}_{1}, \ldots, \underline{x}_{k}$ are normally distributed, but obviously holds for a large class of distributions including the absolutely continuous one-parameter exponential families.

As a measure of the performance of an admissible size- $\alpha$ test $\delta$ we consider its shortcoming

$$
\mathrm{R}_{\delta}(\mu)=\mathrm{R}_{\delta}\left(\mu_{1}, \ldots, \mu_{\mathrm{k}}\right)=\beta_{\alpha}^{+}(\mu)-\beta_{\delta}(\mu)
$$

(cf. section 1.3), or equivalently, if $\mathrm{A}$ is the acceptance region of $\delta$,

$$
R_{\delta}(\mu)=P\left(\left(\underline{x}_{1}, \ldots, \underline{x}_{k}\right) \in A \mid \mu\right)-\Phi\left(u_{\alpha}-\left(\sum_{i=1}^{k} \mu_{i}^{2}\right)^{\frac{1}{2}}\right) .
$$

We shall show that, with some well-defined exceptions, the shortcoming of an admissible test has only one relative and absolute maximum on any half-line through the origin in the positive orthant. In fact this fundamental property holds for a much wider class of distributions and we shall therefore prove a more general theorem.

Let $\underline{x}_{1}, \underline{x}_{2}, \ldots, \underline{x}_{k}$ be independent with joint density

$$
p(x ; \theta)=\prod_{i=1}^{k} C_{i}\left(\theta_{i}\right) h_{i}\left(x_{i}\right)_{\theta}{ }^{\theta}{ } x_{i} \quad, \theta \in \Omega,
$$

with respect to either Lebesgue measure or counting measure, where the sample space $\mathrm{X}$ is the cartesian product of $\mathrm{k}$ intervals (in the absolutely continuous case) or the cartesian product of $\mathrm{k}$ sets of subsequent integers (in the discrete case), and $p(x ; \theta)>0$ for all $x \in X$ and $\theta \in \Omega$. It is assumed that the set $\{\theta \mid \theta \geq 0\}$ is a subset of the interior of the natural parameter space of the exponential family $(2.2 .6)$. We consider testing the hypothesis

$$
\mathrm{H}^{\prime}: \theta=\left(\theta_{1}, \ldots, \theta_{\mathrm{k}}\right)=0
$$

against one-sided alternatives

$$
K^{\prime}: \theta \cdot \geq 0
$$


By a strongly increasing (decreasing) real-valued function of scalar argument we mean a function with a positive (negative) derivative.

\section{Theorem 2.2.2}

Let $\underline{x}_{1}, \underline{x}_{2}, \ldots, \underline{x}_{k}(k \geq 2)$ be distributed according to the exponential family $(2.2 .6)$ and let the assumptions mentioned above be satisfied. Let the functions $h_{1}, h_{2}, \ldots, h_{k}$ be $P_{2}$ (cf. section 1.5). Let $\delta$ be an admissible size- $\alpha$ test of the hypothesis $\mathrm{H}^{\prime}$ against $\mathrm{K}^{\prime}$. For any fixed vector $\left(\zeta_{1}, \ldots, \zeta_{\mathrm{K}}\right)$ with nonnegative coordinates satisfying $\sum \zeta_{i}^{2}=1$ we define

$$
\text { (2.2.7) } \quad f(\rho) \stackrel{\text { def }}{=} R_{\delta}\left(\zeta_{1} \rho, \ldots, \zeta_{k} \rho\right)=\beta_{\alpha}^{+}\left(\zeta_{1} \rho, \ldots, \zeta_{k} \rho\right)-\beta_{\delta}\left(\zeta_{1} \rho, \ldots, \zeta_{k} \rho\right) \text {. }
$$

Then, with the exceptions mentioned below, $f$ has a unique relative maximum on $(0, \infty)$ that is also its absolute maximum. In fact, $f$ decreases strongly away from this maximum on both sides, vanishes for $\rho=0$ and $\rho \rightarrow \infty$, and has a negative second derivative at the maximum.

The exceptions occur in the following two cases:

(i) the test $\delta$ rejects $H^{\prime}$ for large values of $\left[\zeta_{i} x_{i}\right.$, in which case $f$ vanishes identically for all $\rho \geq 0$;

(ii) the test $\delta$ does not involve $\underline{x}_{i}$ for those values of $i$ for which $\zeta_{i}>0$, in which case $f$ is strongly increasing for all $\rho>0$.

We remark that tests of the hypothesis $\theta=\theta^{0}$ against $\theta . \geq \theta^{0}$ reduce to tests of $H^{\prime}$ against $K^{\prime}$ if new parameters $\theta_{i}^{\prime}=\theta_{i}-\theta_{i}^{0}$ are introduced. If the functions $h_{i}$ are $\mathrm{PF}_{2}$, the functions $h_{i}\left(x_{i}\right) \exp \left(\theta_{i}^{0} x_{i}\right)$ arising with this transformation are also $\mathrm{PF}_{2}$.

\section{Proof of theorem 2.2.2}

It follows from lemma 1.4.2 and theorem 1.4.3 that the class of tests with a.e. monotone and convex acceptance regions is essentially complete (cf. section 1.4). Hence it may be assumed that $\delta$ belongs to this class. Moreover, it may be assumed without loss of generality that the critical function $\delta$ is non-decreasing in $\mathrm{x}$ on $\mathrm{X}$, including the boundary of the acceptance region $\mathrm{A}$. This is a consequence of the fact that, if $x^{0} \leq . x^{\prime}$ and $\delta\left(x^{0}\right)>0, \delta\left(x^{\prime}\right)<1$, the power of $\delta$ can uniformly be improved upon because of the strict MLR of $p(x ; \theta)$ in $x$.

First we consider the exceptions to the theorem. In case ( $i$ ) the test $\delta$ is a most powerful (MP) size- $\alpha$ test of $H^{\prime}$ against the alternative $\left(\zeta_{1} \rho, \ldots, \zeta_{k} \rho\right)$ 
for every $\rho>0$. Hence $f(\rho) \equiv 0$ on $(0, \infty)$ in case $(i)$. In all other cases we have $f(\rho)>0$ for all $\rho>0$. In case $(i i)$ the power of $\delta$ against $\left(\zeta_{1} \rho, \ldots, \zeta_{k} \rho\right)$ does not depend on $\rho$ and to prove the assertion for this case we have to show that the envelope power is strongly increasing in $\rho$. The MP size- $\alpha$ test of $H^{\prime}$ against $\left(\zeta_{1} \rho, \ldots, \zeta_{k} \rho\right)$ rejects $H^{\prime}$ for large values of the statistic

$$
\underline{s}=\sum_{i=1}^{k} \zeta_{i} \underline{x}_{i} .
$$

The density of $\underline{\underline{s}}$ is again of exponential family type with parameter $\rho$; let us write its density with respect to the appropriate measure $\lambda$ as

$$
\gamma(\rho) g(s) e^{\rho s}
$$

(cf.(2.2.9)) and let $\delta^{*}(s)$ denote the critical function of the MP test. Then

$$
\beta_{\alpha}^{+}\left(\zeta_{1} \rho, \ldots, \zeta_{k^{\rho}}\right)=\gamma(\rho) \cdot \int \delta^{*}(s) g(s) e^{\rho s} d \lambda(s),
$$

and, since $\delta^{*}$ is non-decreasing, $\exp (\rho s)$ is $\operatorname{ETP}(\rho)$ (cf.section 1.5) and (2.2.8) may be differentiated with respect to $\rho$ under the integral, it follows from theorem 6.3 .4 (c) of S. KARLIN [25] that (2.2.8) is a strongly increasing function of $\rho$.

Disregarding the exceptions (i) and (ii) for the remainder of the proof, we shall first establish that $f(\rho) \rightarrow 0$ for $\rho \rightarrow \infty$. Let $x^{*} \in X-$ clos $(A)$. Since the exceptional case ( $i i)$ has been excluded and $A$ is monotone and convex, there exists a supporting hyperplane

$$
\sum_{i=1}^{k} v_{i} x_{i}=d
$$

of $A$ separating $x^{*}$ and $\operatorname{clos}(A)$ such that $\nu_{i} \geq 0$ for $i=1,2, \ldots, k$ and $\nu_{j} \zeta_{j}>0$ for some $j \in\{1,2, \ldots, k\}$. Writing $\underline{z}=\sum \nu_{i} \underline{x}_{i}$, we find for any $\rho>0$

$\beta_{\delta}\left(\zeta_{1} \rho, \ldots, \zeta_{k} \rho\right) \geq P\left(\underline{z}>d \mid \zeta_{1} \rho, \ldots, \zeta_{k} \rho\right) \geq P\left(\underline{z}>d \mid \zeta_{j} \rho, \zeta_{i} \rho=0\right.$ for $\left.i \neq j\right)=b(d, \rho)$, say. The last inequality is motivated by the fact that $\nu_{i} x_{i}$ is stochastically increasing for increasing $\rho$ for any $i$ for which $\nu_{i} \zeta_{i}>0$. The positivety of the functions $h_{i}$ implies that $b(d, p)>0$. Moreover, under the condition $\zeta_{i} \rho=0$ for $i \neq j$, the distribution of $\underline{z}$ is an exponential family with parameter $\zeta_{j} \rho / \nu_{j}$. It is now easily shown that $b(d, \rho) \rightarrow 1$ for $\rho+\infty$, implying 


$$
\lim _{\rho \rightarrow \infty} \beta_{\delta}\left(\zeta_{1} \rho, \ldots, \zeta_{\mathrm{k}} \rho\right)=1
$$

Hence $f(p) \rightarrow 0$ for $p \rightarrow \infty$. In the discrete case it may happen that no point $\mathrm{x}^{*} \in \mathrm{X}-\operatorname{clos}(\mathrm{A})$ exists (although $0<\alpha<1$ ). In this situation $\delta(\mathrm{x})=0$ for all $\mathrm{x} \in \mathrm{X}$ with the exception of $\mathrm{x}_{\max }=\left(\mathrm{x}_{1, \max }, \ldots, \mathrm{x}_{\mathrm{k}, \max }\right)$, where $\mathrm{x}_{i, \max }$ is the largest value that can be assumed by $\underline{x}_{i}(i=1,2, \ldots, k)$. But in this case $\delta$ is a MP test of $\mathrm{H}^{\prime}$ against $\left(\zeta_{1} \rho, \ldots, \zeta_{\mathrm{k}} \rho\right)$, i.e. we are in the exceptional case (i). Thus we have found that $f(0)=0, f(\rho)>0$ for all $\rho>0$ and $f(\rho) \rightarrow 0$ for $\rho+\infty$. Without loss of generality we assume in the sequel that $\zeta_{i}>0$ for $i=1,2, \ldots, m$ and $\zeta_{i}=0$ for $i=m+1, m+2, \ldots, k(1 \leq m \leq k)$.

First suppose $m \geq 2$. Introducing the random variables

$$
\begin{aligned}
& \underline{\mathrm{y}}_{1}=\zeta_{1} \underline{\mathrm{x}}_{1}, \ldots, \underline{\mathrm{y}}_{\mathrm{m}}=\zeta_{\mathrm{m}} \underline{\mathrm{x}}_{\mathrm{m}}, \underline{\mathrm{y}}_{\mathrm{m}+1}=\underline{\mathrm{x}}_{\mathrm{m}+1}, \ldots, \underline{\mathrm{y}}_{\mathrm{k}}=\underline{\mathrm{x}}_{\mathrm{k}}, \\
& \underline{\mathrm{s}}=\sum_{\mathrm{i}=1}^{\mathrm{m}} \underline{\mathrm{y}}_{\mathrm{i}},
\end{aligned}
$$

and putting

$$
\begin{aligned}
& \gamma(\rho)=\prod_{i=1}^{m} C_{i}\left(\zeta_{i} \rho\right), \\
& h_{i}^{*}\left(y_{i}\right)=h_{i}\left(y_{i} / \zeta_{i}\right), i=1,2, \ldots, m,
\end{aligned}
$$

$\underline{y}_{1}, \underline{y}_{2}, \ldots, \underline{y}_{k}$ have the joint density

$$
\gamma(\rho) \exp \left(\rho \sum_{i=1}^{m} y_{i}\right) \prod_{i=1}^{m} h_{i}^{*}\left(y_{i}\right) \prod_{i=m+1}^{k} h_{i}\left(y_{i}\right)
$$

with respect to a product measure $\lambda_{1} \times \ldots \times \lambda_{k}$, and $\underline{s}$ has the density

$$
\begin{aligned}
& \gamma(\rho) e^{\rho s} \int \ldots \int h_{1}^{*}\left(s-\sum_{i=2}^{m} y_{i}\right) \prod_{i=2}^{m} h_{i}^{*}\left(y_{i}\right) d \lambda_{2}\left(y_{2}\right) \ldots d \lambda_{m}\left(y_{m}\right) \\
& =\gamma(\rho) e^{\rho s} g_{m}(s) \text {, say, }
\end{aligned}
$$

with respect to a measure $\lambda$. The critical function $\delta$ in $x$-space transforms into a critical function $\bar{\delta}$ in $y$-space which remains non-decreasing. The MP size- $\alpha$ test of $\mathrm{H}^{\prime}$ against all alternatives of the form $\left(\zeta_{1} \rho, \ldots, \zeta_{\mathrm{k}} \rho\right), 0<\rho<\infty$, rejects $\mathrm{H}^{\prime}$ for large values of $\underline{s}$; let us again denote its critical function by $\delta^{*}$. We have 


$$
\begin{aligned}
f(p) & =\int \delta^{*}(s) \gamma(\rho) e^{\rho s} g_{m}(s) d \lambda(s)+ \\
& -\int \ldots \delta \delta\left(y_{1}, \ldots y_{k}\right) \gamma(\rho) \exp \left(\rho \sum_{i=1}^{m} y_{i}\right) \prod_{i=1}^{m} h_{i}^{*}\left(y_{i}\right) \prod_{i=m+1}^{k} h_{i}\left(y_{i}\right) d \lambda_{1}\left(y_{1}\right) \ldots d \lambda_{k}\left(y_{k}\right) \\
& =\int \delta^{*}(s) \gamma(\rho) e^{\rho s} g_{m}(s) d \lambda(s)+ \\
& -\int \ldots \delta \delta\left(s-\sum_{i=2}^{m} y_{i}, y_{2}, \ldots, y_{k}\right) \gamma(\rho) e^{\rho s} h_{1}^{*}\left(s-\sum_{i=2}^{m} y_{i}\right) \prod_{i=2}^{m} h_{i}^{*}\left(y_{i}\right) . \\
& =\int_{G}\left[\delta^{*}(s)-\int \ldots \int \delta\left(s-\sum_{i=2}^{m} y_{i}, y_{2}, \ldots, y_{k}\right) \prod_{i=m+1}^{k} h_{i}\left(y_{i}\right) .\right. \\
(2.2 .10) & \left.\quad \frac{h_{1}^{*}\left(s-\sum_{i=2}^{m} y_{i}\right) \prod_{i=2}^{m} h_{i}^{*}\left(y_{i}\right)}{g_{m}(s)} d \lambda_{2}\left(y_{2}\right) \ldots d \lambda_{k}\left(y_{k}\right)\right] \gamma(\rho) e^{\rho s} g_{m}(s) d \lambda(s),
\end{aligned}
$$

where $G$ is the measurable set $\left\{s \mid g_{m}(s)>0\right\}$. The second term between the brackets in (2.2.10) is easily recognized as a version of the conditional expectation of

$$
\int \ldots \int \delta\left(y_{1}, \ldots, \underline{y}_{m}, y_{m+1}, \ldots, y_{k}\right) \prod_{i=m+1}^{k} h_{i}\left(y_{i}\right) d \lambda_{m+1}\left(y_{m+1}\right) . . d \lambda_{k}\left(y_{k}\right)
$$

given $\sum^{m} y_{i}=s$. Since $(2.2 .11)$ is non-decreasing in $\underline{y}_{1}, \mathrm{y}_{2}, \ldots, \mathrm{y}_{\mathrm{m}}$, application of EFRON ${ }^{\prime}$ s theorem (lemma 1.5.5) yields that this conditional expectation is a nondecreasing function of $\mathrm{s}$. Moreover, it is bounded below by 0 and above by 1 . Denoting the expression between brackets in (2.2.10) by $\mathrm{H}_{\mathrm{m}}(\mathrm{s})$, it follows that for any positive constant a the function

$$
\mathrm{H}_{\mathrm{m}}(\mathrm{s})-\mathrm{a}
$$

changes sign at most twice on $G$; furthermore if it does have two changes of sign, the signs occur in the order $(-,+,-)$ for increasing $s$.

So far we have assumed $m \geq 2$. If $m=1$ we simply have $\underline{s}=\underline{y}_{1}$ and we obtain in a similar fashion

$$
\begin{gathered}
f(\rho)=\int_{G}\left[\delta^{*}(s)-\int \ldots \int \delta\left(s, y_{2}, \ldots, y_{k}\right) \underset{i=2}{k} h_{i}\left(y_{i}\right) d \lambda_{2}\left(y_{2}\right) \ldots d \lambda_{k}\left(y_{k}\right)\right] . \\
. \gamma(\rho) e^{\rho s} g_{1}(s) d \lambda(s),
\end{gathered}
$$

where $g_{1}(s)=h_{1}^{*}(s)$. Denoting the form between brackets by $H_{1}(s)$, it is obvious 
that the conclusion previously drawn for $\mathrm{H}_{\mathrm{m}}(\mathrm{s})$-a in case $\mathrm{m} \geq 2$ also holds for $\mathrm{m}=1$.

Since any $\theta \geq 0$ is an interior point of the natural parameter space of (2.2.6), $f$ is certainly twice continuously differentiable and the differentiation may be carried out under the integral. Furthermore $f$ cannot be identically equal to a constant since $f(0)=0$ and $f(\rho)>0$ for $\rho>0$, and $\gamma(\rho) g_{m}(s) \exp (\rho s)$ is $\operatorname{ETP}(\rho)$. These conditions being satisfied, it follows from lemma 1.5 .4 that for any a $>0$ the function

$$
f(\rho)-a=\int_{G}\left\{H_{m}(s)-a\right\} \gamma(\rho) e^{\rho s} g_{m}(s) d \lambda(s)
$$

has at most two zeros counting multiplicities. As $f(\rho)>0$ for $\rho>0$ and $f(\rho)$ tends to zero for $\rho \rightarrow 0$ and $\rho \rightarrow \infty$, the function $f$ has a unique relative maximum on $(0, \infty)$ which is also its absolute maximum. A vanishing derivative of $f$ at some point $0<\rho_{0}<\infty$ other than the point where the maximum is reached would produce at least one double and one single zero of the function $f(\rho)-f\left(\rho_{0}\right)$. Choosing for a the maximum value of $f(\rho)$, a vanishing second derivative at the maximum would produce a triple zero of $f(\rho)-a$. Hence neither of these situations can occur, and all the assertions of the theorem are proved.

Q.E.D.

The conditions of theorem 2.2 .2 are certainly satisfied if $\underline{x}_{1}, \underline{x}_{2}, \ldots, \underline{x}_{k}$ have normal distributions with unit variances and the hypothesis $H$ is tested against $K$, since $h_{i}\left(x_{i}\right)=\exp \left(-\frac{1}{2} x_{i}^{2}\right)$ in this case and this function is PF. A simpler proof for this particular case was given in [60].

The crucial condition of the theorem that $h_{i}$ is $\mathrm{PF}_{2}$ is satisfied by many exponential families occuring in well-knowm combination problems, as is demonstrated by the following examples.

Example 2.2.1

The combination of $\mathrm{k}$ sign tests. In this case the random variables $\underline{x}_{1}, \underline{x}_{2}, \ldots, \underline{x}_{k}$ have binomial distributions

$$
P\left(\underline{x}_{i}=x_{i}\right)=\left(1+e^{\theta}\right)^{-n_{i}}\left(\begin{array}{l}
n_{i} \\
x_{i}
\end{array}\right) e^{\theta_{i} x_{i}}, i=1,2, \ldots, k,
$$

where $\theta_{i}=\log \left(p_{i} /\left(1-p_{i}\right)\right)$, and to prove that $\left(\begin{array}{c}n_{i}^{i} \\ x_{i}\end{array}\right)$ is $P F_{2}$ we have to show that

$$
\left(\begin{array}{c}
n_{i} \\
x_{1}-y_{1}
\end{array}\right)\left(\begin{array}{c}
n_{i} \\
x_{2}-y_{2}
\end{array}\right)-\left(\begin{array}{c}
n_{i} \\
x_{1}-y_{2}
\end{array}\right)\left(\begin{array}{c}
n_{i} \\
x_{2}-y_{1}
\end{array}\right) \geq 0
$$


for all integers $0 \leq \mathrm{x}_{1}<\mathrm{x}_{2} \leq \mathrm{n}_{\mathrm{i}}$ and $0 \leq \mathrm{y}_{1}<\mathrm{y}_{2} \leq \mathrm{n}_{\mathrm{i}}$. Putting $\mathrm{x}_{1}-\mathrm{y}_{1}=\mathrm{a}$, $x_{2}-y_{2}=b$ and $x_{2}-x_{1}=r$, where $r>0$ and $b-a<r$, we find

$$
\left(\begin{array}{l}
n_{i} \\
a
\end{array}\right)\left(\begin{array}{c}
n_{i} \\
b
\end{array}\right) /\left(\begin{array}{c}
n_{i} \\
b-r
\end{array}\right)\left(\underset{a+r}{n_{i}}\right)=\frac{a+r}{b-r+1} \cdots \frac{a+r}{b} \frac{n_{i}-b+1}{n_{i}^{-a+r+1}} \cdots \frac{n_{i}-b+r}{n_{i}-a}>1,
$$

proving the desired inequality. Hence the theorem holds in this case if the hypothesis $p=\left(p_{1}, \ldots, p_{k}\right)=\left(\frac{1}{2}, \ldots, \frac{1}{2}\right)$ is tested against $p \cdot \geq\left(\frac{1}{2}, \ldots, \frac{1}{2}\right)$.

\section{Example 2.2.2}

The combination of $\mathrm{k} 2 \times 2$ tables :

$\begin{array}{rccc} & \text { Success } & \text { Failure } & \text { Total } \\ \text { First series } & \underline{a}_{i} & \underline{c}_{i} & m_{i} \\ \text { Second series } & \underline{b}_{i} & \underline{d}_{i} & n_{i} \\ \text { Total } & \underline{r}_{i} & \underline{s}_{i} & m_{i}+n_{i}, i=1,2, \ldots, k\end{array}$

The optimal conditional test for testing equality of the probabilities $p_{i, 1}$ and $p_{i, 2}$ of success in the first and second series respectively against the alternative $p_{i, 1}>p_{i, 2}$ rejects for large values of $a_{i}$. Defining

$$
\theta_{i}=\log \left\{p_{i, 1}\left(1-p_{i, 2}\right) /\left(p_{i, 2}\left(1-p_{i, 1}\right)\right)\right\},
$$

the conditional distribution of $\underline{a}_{i}$ given $\underline{r}_{i}=r_{i}$ and $\underline{s}_{i}=s_{i}$ is

$$
P\left(\underline{a}_{i}=a_{i} \mid \theta_{i}, r_{i}, s_{i}\right)=\left(\begin{array}{l}
m_{i} \\
a_{i}
\end{array}\right)\left(\begin{array}{c}
n_{i} \\
r_{i}-a_{i}
\end{array}\right) e^{\theta_{i} a_{i}} / \sum_{j}\left(\begin{array}{l}
m_{j} \\
j
\end{array}\right)\left(\begin{array}{c}
n_{i} \\
r_{i}-j
\end{array}\right) e^{\theta_{i} j},
$$

and we have to show that $\left({ }_{a_{i}}^{m_{i}}\right)\left(\begin{array}{c}n_{i} \\ r_{i}-a_{i}\end{array}\right)$ is $P F_{2}$ (as a function of $a_{i}$ ). Since the product of two $\mathrm{PF}_{2}$ functions ${ }^{i}$ is again $\mathrm{PF}_{2}$, it is sufficient to prove that both factors are $\mathrm{PF}_{2}$ and this follows as in example 2.2.1.

Hence theorem 2.2.2 also applies in this case.

Example 2.2.3

Let $\underline{x}_{1}, \underline{x}_{2}, \ldots, \underline{x}_{k}$ be gamma distributed with densities

$$
\left(\beta_{i}^{i} / \Gamma\left(\gamma_{i}\right)\right) x_{i}^{\gamma_{i}^{-1}} e^{-\beta_{i} x_{i}}\left(x_{i}>0\right), i=1,2, \ldots, k,
$$


where $\gamma_{i}$ is the shape parameter and $\beta_{i}$ the scale parameter. Suppose we wish to test $\beta=\left(\beta_{1}, \ldots, \beta_{k}\right)=\beta^{0}$ against $\beta \leq \beta^{0}$, assuming $\gamma_{1}, \gamma_{2}, \ldots, \gamma_{k}$ to be known. Writing $\theta_{i}=\beta_{i, 0}-\beta_{i}$, the density (2.2.13) transforms to the canonical form (2.2.6) with

$$
h_{i}\left(x_{i}\right)=x_{i}^{\gamma_{i}^{-1}} e^{-\beta} i, x_{i}, i=1,2, \ldots, k \text {. }
$$

Since $d^{2} \log h_{i}\left(x_{i}\right) / d x_{i}^{2}=-\left(\gamma_{i}-1\right) x_{i}^{-2}$ is nonpositive for $\gamma_{i} \geq 1, h_{i}\left(x_{i}\right)$ is $P F_{2}$ for $\gamma_{i} \geq 1$ (cf. lemma 1.5.2). Hence theorem 2.2.2 holds for this testing problem, provided $\gamma_{i} \geq 1(i=1,2, \ldots, k)$.

We have thus shown that for a large class of combination problems the shortcoming of an admissible test has only one maximum on any half-line through the origin in the positive orthant. Returning now to the case of normal random variables, we present a lemma stating that the maxima of the shortcoming along these half-lines cannot be reached for alternatives close to the origin if $\alpha$ is small.

\section{Lemma 2.2.1}

Let $k \geq 2$ and let in the normal case $\delta$ be any admissible size- $\alpha$ test of $H$ against $K(0<\alpha<1)$. Then the shortcoming $f(\rho)$ of $\delta$ along any half-line in the positive orthant defined by (2.2.7) reaches its unique maximum for some $\rho>u_{\alpha}$, if $f(\rho)$ has a maximum at all.

Proof:

We consider the shortcoming of $\delta$ along the half-line $\mu_{1}=\zeta_{1} \rho, \mu_{2}=\zeta_{2} \rho, \ldots \mu_{k}=\zeta_{k} \rho$, $\rho>0$, where $\zeta_{i} \geq 0(i=1,2, \ldots, k)$ and $\sum \zeta_{i}^{2}=1$. Consider an orthogonal transformation in $\mathbb{R}^{k}$, carrying $\underline{x}_{1}, \underline{x}_{2}, \ldots, \underline{x}_{k}$ into $\underline{y}_{1}, \underline{y}_{2}, \ldots, \underline{y}_{k}$, where $\underline{y}_{1}=\sum_{i=1} \zeta_{i} \underline{x}_{i}$. Then $\underline{y}_{1}, \underline{y}_{2}, \ldots, \underline{y}_{k}$ are independent and, if $\underline{E x}_{i}=\mu_{i}=\zeta_{i} \rho$ for $i=1,2, \ldots, i=1$, then $\underline{y}_{1}$ is $\mathbb{N}(\rho, 1)$ and $y_{i}$ is $N(0,1)$ for $i=2,3, \ldots, k$. Let $A$ denote the acceptance region of $\delta$ in $\mathrm{x}$-space and let $\mathrm{B}$ be its map into $\mathrm{y}$-space. Consider two points $\left(\mathrm{y}_{1}, \mathrm{y}_{2}, \ldots, \mathrm{y}_{\mathrm{k}}\right)$ and $\left(\mathrm{y}_{1}^{\prime}, \mathrm{y}_{2}, \ldots, \mathrm{y}_{\mathrm{k}}\right)$ with $\mathrm{y}_{1}^{\prime}<\mathrm{y}_{1}$, in $\mathrm{y}$-space, corresponding to points $\left(\mathrm{x}_{1}, \mathrm{x}_{2}, \ldots, \mathrm{x}_{\mathrm{k}}\right)$ and $\left(x_{1}^{\prime}, x_{2}^{1}, \ldots, x_{k}^{\prime}\right)$ respectively in $x$-space. If $\left(y_{1}, y_{2}, \ldots, y_{k}\right) \in B$ then $\left(x_{1}, x_{2}, \ldots, x_{k}\right)$ $\epsilon A$ and inverting the transformation we find

$$
x_{i}-x_{i}^{\prime}=\zeta_{i}\left(y_{1}-y_{1}^{\prime}\right) \geq 0 .
$$

Hence by lemma $1.4 .6\left(x_{1}^{\prime}, x_{2}^{\prime}, \ldots, x_{k}^{\prime}\right) \in A$, implying $\left(y_{1}^{\prime}, y_{2}, \ldots, y_{k}\right) \in B$. Therefore we can write $B$ in the form 


$$
B=\left\{\left(y_{1}, \ldots y_{k}\right) \mid y_{1}<b\left(y_{2}, \ldots, y_{k}\right)\right\} .
$$

Thus, if $\phi$ denotes the standard normal density,

$$
f(\rho)=\int \ldots \int \Phi\left(b\left(y_{2}, \ldots y_{k}\right)-\rho\right) \prod_{i=2}^{k} \phi\left(y_{i}\right) d y_{2} \ldots d y_{k}-\Phi\left(u_{\alpha}-\rho\right),
$$

and hence, differentiating with respect to $\rho$,

$$
f^{\prime}(\rho)=\int \ldots \int\left[\phi\left(u_{\alpha}-\rho\right)-\phi\left(b\left(y_{2}, \ldots, y_{k}\right)-\rho\right)\right] \underset{i=2}{k} \phi\left(y_{i}\right) d y_{2} \ldots d y_{k} .
$$

It follows that $f^{\prime}\left(u_{\alpha}\right)>0$, since $\phi(0)>\phi(z)$ for all $z \neq 0$. The assertion of the lemma is now an immediate consequence of theorem 2.2.2.

If $k=2$, i.e. if only two random variables $\underline{x}_{1}$ and $\underline{x}_{2}$ are involved, a stronger theorem can be obtained. First we introduce some notation. Let $\rho$ and $n$ denote polar coordinates in $\left(\mu_{1}, \mu_{2}\right)$-space, i.e.

$$
\mu_{1}=\rho \cos \eta \quad, \mu_{2}=\rho \sin n \quad .
$$

By lemma 1.4 .6 we may assume that an admissible combination procedure for testing $H$ against $K$ (for $k=2$ ) has an acceptance region of the form $\left\{\left(x_{1}, x_{2}\right) \mid x_{2}<a\left(x_{1}\right)\right\}$, where $a$ is a non-increasing concave function on the interval where $a\left(x_{1}\right)>-\infty$ (as a boundary case we have $a\left(x_{1}\right)=+\infty$ or $-\infty$ for $x_{1}<u_{\alpha}$ and $x_{1}>u_{\alpha}$ respectively).

Theorem 2.2.3

If $k=2$, the shortcoming of any admissible combination procedure for testing $H$ against $K$ has a finite number of absolute maxima.

Proof:

We start by assuming that the procedure involves both $\underline{x}_{1}$ and $\underline{x}_{2}$ and is not linear. Let $R_{\delta}^{*}$ denote the shortcoming of the test $\delta$ as a function of $\rho$ and $n$,

$$
\begin{aligned}
\mathrm{R}_{\delta}^{*}(\rho, \eta) & =\mathrm{P}\left(\underline{x}_{2}<a\left(\underline{x}_{1}\right) \mid \rho, n\right)-\Phi\left(u_{\alpha}-\rho\right) \\
& =\int \Phi\left(a\left(x_{1}\right)-\rho \sin \eta\right) \phi\left(x_{1}-\rho \cos \eta\right) d x_{1}-\Phi\left(u_{\alpha}-\rho\right)
\end{aligned}
$$

(cf.(2.2.5)), where $\phi$ denotes the standard normal density. Since $\phi$ and $\Phi$ are analytic functions, $R_{\delta}^{*}$ is analytic in $\rho$ and in $n$ for $\rho \geq 0$ and $0 \leq n \leq \frac{1}{2} \pi$, cf. [52]. (Here analyticity of a real-valued function of a real variable on an interval may be taken to mean that the function can be expanded in a power series 
converging on that interval). By theorem 2.2.2 there exists a unique value $\rho(\eta)$ for each $0 \leq n \leq \frac{1}{2} \pi$ such that

$$
\left.\frac{\partial}{\partial \rho} R_{\delta}^{*}(\rho, n)\right|_{\rho=\rho(n)}=0
$$

and

$$
\left.\frac{\partial^{2}}{\partial \rho^{2}} R_{\delta}^{*}(\rho, n)\right|_{\rho=\rho(n)}<0 .
$$

Application of the implicit function theorem yields that $\rho(n)$ is analytic for $0 \leq n \leq \frac{1}{2} \pi$ and hence so is $g(n)=R_{\delta}^{*}(\rho(n), n)$.

Theorem 2.2.2 asserts that the absolute maxima of $R_{\delta}^{*}$ lie on the curve $\rho=\rho(n)$. If $R_{\delta}^{*}$ and hence $g$ would have an infinite number of absolute maxima, $g(n)$ would assume the same value infinitely often and hence would be equal to a constant on $\left[0, \frac{1}{2} \pi\right]$ because of its analyticity. However, this is impossible since $R_{\delta}^{*}$ has a local maximum at the boundary point $n=0, \rho=\rho(0)$, because of (2.2.15), $(2.2 .16)$ and

$$
\left.\frac{\partial}{\partial \eta} R_{\delta}^{*}(\rho, n)\right|_{n=0}=-\rho \int \phi\left(a\left(x_{1}\right)\right) \phi\left(x_{1}-\rho\right) d x_{1}<0 .
$$

It remains to consider the two exceptions of theorem 2.2.2. If the procedure $\delta$ depends on both $\underline{x}_{1}$ and $\underline{x}_{2}$ but is linear, i.e. rejects $H$ if $v_{1} \underline{x}_{1}+v_{2} \underline{x}_{2} \geq d$ for positive $v_{1}$ and $\nu_{2}$, then the assertion of theorem 2.2 .2 continues to hold for every half-line $\rho \geq 0, n=n_{0}$ with $0 \leq n_{0} \leq \frac{1}{2} \pi, n_{0} \neq n_{1}$, where $\operatorname{tn} n_{1}=\nu_{2} / \nu_{1}$. Hence in this case we have analyticity of $\rho(n)$ and $g(n)$ on both $\left[0, n_{1}\right)$ and $\left(n_{1}, \frac{1}{2} \pi\right]$. By partial integration we find

$$
\begin{aligned}
\left.\frac{\partial}{\partial \eta} R_{\delta}^{*}(\rho, n)\right|_{n=\frac{1}{2} \pi} & =-\rho \int \Phi\left(a\left(x_{1}\right)-\rho\right) \phi^{\prime}\left(x_{1}\right) d x_{1} \\
& =-\rho \int \phi\left(a\left(x_{1}\right)-\rho\right) \phi\left(x_{1}\right) a^{\prime}\left(x_{1}\right) d x_{1}>0
\end{aligned}
$$

since $a^{\prime}\left(x_{1}\right) \equiv-v_{1} / v_{2}<0$ in this case. It now follows from (2.2.15) through (2.2.18) by a similar argument that $R_{\delta}^{*}$ can only have a finite number of absolute maxima.

Finally, if the test $\delta$ does not depend on both $\underline{x}_{1}$ and $\underline{x}_{2}$, e.g. rejects $H$ if $\underline{x}_{1} \geq u_{\alpha}$, then $R_{\delta}\left(\mu_{1}, \mu_{2}\right)$ is a strongly increasing function of $\mu_{2}$ for each value of $\mu_{1}$ and hence $R_{\delta}$ does not possess any absolute maxima at all. Q.E.D. 
It is unknown whether the assertion of theorem 2.2.3 continues to hold for $k>2$. Generalization of the theorem to one-parameter exponential families under the conditions of theorem 2.2.2 is not immediate, even if the functions $\mathrm{h}_{i}$ in (2.2.6) are analytic, since the relations (2.2.17) and (2.2.18) are not automatically satisfied and are not so easily replaced by other relations ensuring that the function $\mathrm{R}_{\delta}^{*}(\rho, n)$ is not constant on the curve $\rho=\rho(n)$.

\subsection{THE MOST STRINGENT TEST}

As a UMP test, a UMP unbiased or UMP invariant test for testing $H$ against $\mathrm{K}$ (cf.section 2.2) do not exist, we shall try to determine the most stringent (MS) size- $\alpha$ test for this problem with the aid of a least favorable (LF) prior distribution. A test that is invariant under permutations of $\underline{x}_{1}, \underline{x}_{2}, \ldots, \underline{x}_{k}$ will be called symmetric.

Lemma 2.3.1

For any size $0<\alpha<1$ an essentially unique MS test of $H$ against $K$ and a $L F$ prior distribution exists. The MS test is symmetric.

Proof:

As the permutation group of $\mathrm{k}$ elements is finite, the existence of a symmetric MS size- $\alpha$ test follows from lemma 1.3.1. This procedure is of course also MS relative to the class $\mathcal{J}(\alpha)$ of admissible symmetric size- $\alpha$ procedures. For each test in $f_{(\alpha)}$ the point $x=\left(x_{1}, \ldots, x_{k}\right)=(b, \ldots, b)$, where $\Phi(b)=(1-\alpha)^{1 / k}$, must lie either outside its acceptance region $A$ or on the boundary of A. For otherwise, by lemma 1.4.6, A would contain the set $\left\{x \mid x_{i} \leq b+\varepsilon, i=1,2, \ldots, k\right\}$ for some $\varepsilon>0$ and the size of the test would be smaller than $\alpha$. Also the symmetry of A together with lemma 1.4 .6 guarantees that A has a supporting hyperplane $\sum \mathrm{x}_{i}=\mathrm{d}(\mathrm{d} \leq \mathrm{kb})$ and hence that for every test in $\mathcal{f}(\alpha)$ the acceptance region $A$ is contained in the set $\left\{x \mid \sum_{i} x_{i} \leq k b\right\}$. Therefore we have for every
test $\delta$ in $J(\alpha)$

$$
\begin{aligned}
R_{\delta}(\mu) & \leq P\left(\sum_{i=1}^{k} \underline{x}_{i} \leq k b \mid \mu\right)-\Phi\left(u_{\alpha}-\left(\sum_{i=1}^{k} \mu_{i}^{2}\right)^{\frac{1}{2}}\right) \\
& =\Phi\left(k^{-\frac{1}{2}}\left(k b-\sum_{i=1}^{k} \mu_{i}\right)\right)-\Phi\left(u_{\alpha}-\left(\sum_{i=1}^{k} \mu_{i}^{2}\right)^{\frac{1}{2}}\right),
\end{aligned}
$$


which tends to zero whenever $\sum_{i} \mu_{i}^{2} \rightarrow \infty$ and $\mu \geq 0$.

Let $\mathrm{R}_{\alpha}^{+}$denote the size- $\alpha$ minimax shortcoming (cf.section 1.3 ). Since $\mathrm{R}_{\alpha}^{+}>0$, it follows from (2.3.1) that there exists a positive number $\mathrm{q}$ such that for every test $\delta \in \mathcal{J}(\alpha)$

$$
R_{\delta}(\mu)<\frac{1}{2} R_{\alpha}^{+} \text {for } \sum_{i=1}^{k} \mu_{i}^{2}>q, \mu \geq 0 .
$$

Hence for every $\delta \in S(\alpha)$ the shortcoming assumes its absolute maxima (which are $\geq R_{\alpha}^{+}$) only on the set

$$
\Omega_{q}=\left\{\mu \mid \sum_{i=1}^{k} \mu_{i}^{2} \leq q, \mu \geq 0\right\} .
$$

Now consider the problem of testing $H$ against the alternative that $\mu \in \Omega, \mu \neq 0$. Obviously the MS test for the original problem (which is a member of $\rho(\alpha)$ ) is also MS for this new problem. However, as the parameter space of the new problem is compact, there exists a LF prior distribution $\xi$ for this new problem (cf. section 1.3). Hence the MS test considered must be Bayes with respect to $\xi$ and $\xi$ is thus also LF for the original problem.

As every MS size- $\alpha$ test is Bayes relative to $\xi$, the essential uniqueness of the MS test follows from the fact that the family of normal distributions with unit variance is boundedly complete, implying that any Bayes test is essentially unique.

This lemma can also be generalized to exponential families. Let $\underline{x}_{1}, \underline{x}_{2}, \ldots, \underline{x}_{k}$ have the joint density

$$
p(x ; \theta)=\prod_{i=1}^{k} C\left(\theta_{i}\right) h\left(x_{i}\right) e^{\theta_{i} x_{i}} \quad, \theta \geq 0,
$$

with respect to either Lebesgue measure or counting measure; note that the marginal distributions of $\underline{x}_{1}, \underline{x}_{2}, \ldots, \underline{x}_{k}$ are of the same type. Then lemma 2.3 .1 also holds true for testing the hypothesis $H^{\prime}$ against $K^{\prime}$ (cf. section 2.2). The proof remains literally the same (replacing $\mu_{i}$ by $\theta_{i}$ ) with the exception of the inequality (2.3.1) and its derivation. However, by the same line of argument that we used previously in the proof of theorem 2.2.2 it can be shown that the shortcoming of the symmetric MS test tends uniformly to zero for $\sum \theta_{i}^{2} \rightarrow \infty$ and $\theta \geq 0$. 
We return again to the combination of normal random variables. As a $L F$ prior distribution assigns probability one to the set of points for which the shortcoming of the MS test assumes its absolute maximum, we have the following corollary to theorem 2.2.3.

\section{Corollary 2.3.1}

For $k=2$ and any $0<\alpha<1$ a LF prior distribution for the problem of testing $\mathrm{H}$ against $\mathrm{K}$ assigns probability one to a finite point set.

W. SCHAAFSMA ( $[46]$ p.538) conjectured that corollary 2.3.1 also holds for general $\mathrm{k}$.

Now let us, for a moment, restrict the alternatives to the set

$$
\Omega_{\text {restr }}=\bigcup_{j=1}^{k}\left\{\mu \mid \mu_{j}>0, \mu_{i}=0 \text { for all } i \neq j\right\} \text {, }
$$

and consider tests of $\mathrm{H}$ against the restricted alternative

$$
\widetilde{K}: \mu \in \Omega_{\text {restr }} \text {. }
$$

It is easily verified that lemma 2.3.1 also holds for testing $H$ against the alternative $\tilde{K}$. For this problem we have the following result.

\section{Theorem 2.3.1}

For each $0<\alpha<1$ there exists a unique size- $\alpha$ combination procedure $\delta$ that rejects $\mathrm{H}$ if

$$
\text { (2.3.4) } \quad \sum_{i=1}^{k} \exp \left[r(\alpha) \underline{x}_{i}\right] \geq c(\alpha)
$$

and for which the shortcoming $R_{\delta_{\alpha}}$ assumes its absolute maxima on the set $\Omega_{\text {restr }}$ at the $k$ points $\mu(1), \mu(2), \ldots \ldots, \mu(k)$ of the form

$$
\mu_{i}^{(i)} r(\alpha), \mu_{j}^{(i)}=0 \text { for } j \neq i, \quad i=1,2, \ldots, k .
$$

This test is the essentially unique MS test for testing $H$ against $\widetilde{K}$ at level $\alpha$. If, for a certain $\alpha, R_{\delta_{\alpha}}(r(\alpha), 0, \ldots, 0)$ is also the maximum value of $R_{\delta_{\alpha}}$ on the entire parameter space $\{\mu \mid \mu \geq 0\}$, then this test is also MS for testing $\mathrm{H}$ against $\mathrm{K}$.

\section{Proof:}

By the analogue of lemma 2.3.1 there exists an essentially unique MS test for testing $\mathrm{H}$ against $\widetilde{\mathrm{K}}$ at level $\alpha$, which is symmetric and Bayes with respect to 
a LF prior distribution concentrated on $\Omega_{\text {restr }}$. Since this procedure is admissible for the original problem and depends on all variables $\underline{x}_{1}, \underline{x}_{2}, \ldots, \underline{x}_{k}$ because of its symmetry, its shortcoming has exactly one maximum on each of the half-lines of $\Omega_{\text {restr }}$ by theorem 2.2.2. The symmetry of the test implies that the shortcoming is symmetric about the line $\mu_{1}=\mu_{2}=\ldots=\mu_{k}$ and hence it assumes the same maximum value on the $k$ half-lines of $\Omega_{\text {restr }}$ at the points $\mu_{i}(i)=r$ (say),$\mu_{j}^{(i)}=0$ for $j \neq i, i=1,2, \ldots, k$. It follows that the $L F$ prior distribution concentrates on those $\mathrm{k}$ points; denote the $\mathrm{LF}$ prior probabilities by $\mathrm{p}_{1}, \mathrm{p}_{2}, \ldots, \mathrm{p}_{\mathrm{k}}$ $\left(\sum p_{i}=1\right)$. Application of the NEYMAN-PEARSON lemma yields that the MS test for the restricted problem rejects $H$ if

$$
\sum_{i=1}^{k} p_{i} e^{r x_{i}} \geq c .
$$

The symmetry of the critical region of the MS test now implies that $p_{1}=p_{2}=$ $\ldots=p_{k}=1 / k$. Hence the test $(2.3 .4)$ is a MS size- $\alpha$ test of $H$ against $\tilde{K}$ if its size is $\alpha$ and the maxima of its shortcoming on $\Omega_{\text {restr }}$ are reached at the $k$ points defined by (2.3.5). Since MS size- $\alpha$ tests are essentially unique, the test (2.3.4) satisfying these requirements is unique. The second part of the theorem is obvious.

Q.E.D.

In the sequel we shall call tests that reject $H$ if

$$
\sum_{i=1}^{k} e^{r \underline{x}_{i}} \geq c
$$

exponential combination procedures with parameters $r$ and $c$.

Theorem 2.3.1 also holds if the random variables $\underline{x}_{1}, \underline{x}_{2}, \ldots, \underline{x}_{k}$ are distributed according to the exponential family of densities (2.3.2) and the hypothesis $\mathrm{H}^{\prime}$ is to be tested against the alternative $\mathrm{K}^{\prime}$ (or its restriction $\tilde{\mathrm{K}}^{\prime}$ ), provided the conditions of theorem 2.2.2 are satisfied.

We now turn to the question whether exponential combination procedures are MS for testing $H$ against $K$, for appropriate values of $r$ and $c$. At this point the usefulness of theorem 2.3.1 depends heavily on our ability to verify the condition in the last part of theorem 2.3.1 for a given value of $\alpha$. SCHAAFSMA tried to solve this question by computing the maximum shortcoming of the test (2.3.4) on a large number of half-lines in the positive orthant and comparing these with $R_{\delta}(r(\alpha), 0, \ldots, 0)$, which is rather cumbersome and, from a theoretical point of view, unsatisfactory. If the combination problem involves only two 
random variables ( $i . e . k=2$ ) a more satisfactory solution is possible. For more than two variables the problem is still theoretically unsolved and we only present a conjecture.

We introduce some more notation. Let A denote the (nonrandomized) acceptance region of an admissible symmetric test of $\mathrm{H}$ against $\mathrm{K}$. Then we may write

$$
A=\left\{\left(x_{1}, \ldots, x_{k}\right) \mid x_{1}<a\left(x_{2}, \ldots, x_{k}\right)\right\},
$$

where the function $a$ is symmetric in its $k-1$ arguments and is non-increasing and concave in each of its arguments by lemma 1.4.6. We make the assumption that a has continuous first order partial derivatives on the interval where a is finite. Then there exists a function $x_{0}\left(x_{3}, \ldots, x_{k}\right)$ such that

$$
a\left(x_{0}\left(x_{3}, \ldots, x_{k}\right), x_{3}, \ldots, x_{k}\right) \equiv x_{0}\left(x_{3}, \ldots, x_{k}\right) \text {; }
$$

for $k=2$ this function reduces to a constant $x_{0}$.

The symmetry of A also implies that

$$
a\left(a\left(x_{2}, \ldots, x_{k}\right), x_{3}, \ldots, x_{k}\right) \equiv x_{2}
$$

Define

$$
\begin{aligned}
g\left(x_{2}, \ldots, x_{k}\right) & \stackrel{\text { def }}{=} \frac{1}{2} \frac{\partial}{\partial x_{2}}\left(x_{2}^{2}+a^{2}\left(x_{2}, \ldots, x_{k}\right)\right) \\
& =x_{2}+a\left(x_{2}, \ldots, x_{k}\right) \cdot \frac{\partial}{\partial x_{2}} a\left(x_{2}, \ldots, x_{k}\right) .
\end{aligned}
$$

Then the following two lemmata will prove helpful.

\section{Lemma 2.3.2}

Let $\mathrm{k} \geq 2$, let $\delta$ be an admissible symmetric test of $\mathrm{H}$ against $\mathrm{K}$ and let the function $g$ be defined by (2.3.9). If $g$ is nonpositive on the interval $-\infty<x_{2}<x_{0}\left(x_{3}, \ldots, x_{k}\right)$ for all $x_{3}, \ldots, x_{k}$ for which $g$ is defined, the maximum shortcoming of the test $\delta$ can only be assumed on the set $\Omega_{\text {restr }}$.

Proof:

Let $\mu_{3}, \ldots, \mu_{k}$ be fixed and let $\rho, n$ denote polar coordinates in $\left(\mu_{1}, \mu_{2}\right)$-space : $\mu_{1}=\rho \cos n, \mu_{2}=\rho \sin n, \rho>0,0 \leq n \leq \frac{1}{2} \pi$. Let $R_{\delta}^{*}$ denote the shortcoming of the test $\delta$ as a function of $\rho, n, \mu_{3}, \ldots, \mu_{k}$. We shall study the behavior of $R_{\delta}^{*}$ for fixed $\rho>0$ (and fixed $\mu_{3}, \ldots, \mu_{k}$ ) as a function of $n$. Since the shortcoming is symmetric about $n=\pi / 4$, we restrict attention to the interval $0 \leq n \leq \pi / 4$. 
We have

$$
\begin{aligned}
& R_{\delta}^{*}\left(\rho, n, \mu_{3}, \ldots, \mu_{k}\right)=\int \ldots \int \phi\left(x_{1}-\rho \cos \eta\right)\left(x_{2}-\rho \sin \eta\right) \underset{i=3}{k} \phi\left(x_{i}-\mu_{i}\right) d x_{1} \ldots d x_{k}+ \\
& x_{1}<a\left(x_{2}, \ldots, x_{k}\right) \\
& -\Phi\left(u_{\alpha}-\left(\rho^{2}+\sum_{i=3}^{k} \mu_{i}^{2}\right)^{\frac{1}{2}}\right) \\
& =\int \ldots \int \Phi\left(a\left(x_{2}, \ldots, x_{k}\right)-\rho \cos n\right) \phi\left(x_{2}-\rho \sin r_{1}\right) \underset{i=3}{k} \phi\left(x_{i}-\mu_{i}\right) d x_{2} \ldots d x_{k}+ \\
& -\Phi\left(u_{\alpha}-\left(\rho^{2}+\sum_{i=3}^{k} \mu_{i}^{2}\right)^{\frac{1}{2}}\right)
\end{aligned}
$$

and hence

$$
\frac{\partial}{\partial \eta} R_{\delta}^{*}\left(\rho, n, \mu_{3}, \ldots, \mu_{k}\right)=
$$

$=\rho \int \ldots \int\left\{\sin n \cdot \phi\left(a\left(x_{2}, \ldots, x_{k}\right)-\rho \cos \eta\right) \phi\left(x_{2}-\rho \sin r_{1}\right)-\cos n \cdot\right.$

$$
\text { - } \left.\Phi\left(a\left(x_{2}, \ldots, x_{k}\right)-\rho \cos \eta\right) \phi^{\prime}\left(x_{2}-\rho \sin \eta\right)\right\} \underset{i=3}{k} \phi\left(x_{i}-\mu_{i}\right) d x_{2} \ldots d x_{k}
$$

$=\rho \int \ldots \int\left\{\sin \eta+\frac{\partial}{\partial x_{2}} a\left(x_{2}, \ldots, x_{k}\right) \cos \eta\right\} \phi\left(a\left(x_{2}, \ldots, x_{k}\right)-\rho \cos \eta\right)$.

$$
. \phi\left(x_{2}-\rho \sin \eta\right) \prod_{i=3}^{k} \phi\left(x_{i}-\mu_{i}\right) d x_{2} \ldots d x_{k}
$$

$=\frac{\rho e^{-\frac{1}{2} \rho^{2}}}{2 \pi} \int \ldots \int\left\{\sin n+\frac{\partial}{\partial x_{2}} a\left(x_{2}, \ldots, x_{k}\right) \cos n\right\} e^{\rho\left\{x_{2} \sin n+a\left(x_{2}, \ldots, x_{k}\right) \cos n\right\}}$.

$$
\text { - } e^{-\frac{1}{2}\left\{x_{2}^{2}+a^{2}\left(x_{2}, \ldots, x_{k}\right)\right\}} \underset{i=3}{k} \phi\left(x_{i}-\mu_{i}\right) d x_{2} \ldots d x_{k}
$$

$=\frac{e^{-\frac{1}{2} \rho^{2}}}{2 \pi} \int \ldots \int\left\{\sin n+\quad a\left(x_{2}, \ldots, x_{k}\right) \cos n\right\} e^{\rho\left\{x_{2} \sin n+a\left(x_{2}, \ldots, x_{k}\right) \cos n\right\}}$.

$$
\text { - } e^{-\frac{1}{2}\left\{x_{2}^{2}+a^{2}\left(x_{2}, \ldots, x_{k}\right)\right\}} \prod_{i=3}^{k} \phi\left(x_{i}-\mu_{i}\right) d x_{2} \ldots d x_{k}
$$

$=\frac{e^{-\frac{1}{2} \rho^{2}}}{2 \pi} \int \cdots \int g\left(x_{2}, \ldots, x_{k}\right) e^{\rho\left\{x_{2} \sin \eta+a\left(x_{2}, \ldots, x_{k}\right) \cos \eta\right\}}$.

$$
. e^{-\frac{1}{2}\left\{x_{2}^{2}+a^{2}\left(x_{2}, \ldots, x_{k}\right)\right\}} \underset{i=3}{k} \phi\left(x_{i}-\mu_{i}\right) d x_{2} \ldots d x_{k}
$$


by repeated partial integration with respect to $x_{2}$. By the substitutions $x_{2}=a\left(x_{2}^{\prime}, x_{3}, \ldots, x_{k}\right)$ and $x_{2}^{\prime}=a\left(x_{2}, \ldots x_{k}\right)$ and relation (2.3.8) we may change the integral over the region $-\infty<x_{0}<\infty$ into an integral over the region $-\infty<x_{2}<x_{0}\left(x_{3}, \ldots, x_{k}\right)$ and obtain ${ }^{2}$

$$
\frac{\partial}{\partial n} R_{\delta}^{*}\left(\rho, \eta, \mu_{3}, \ldots, \mu_{k}\right)=
$$

$=\frac{e^{-\frac{1}{2} \rho 2}}{2 \pi} \int \ldots \int\left[\int_{-\infty}^{x_{0}\left(x_{3}, \ldots, x_{k}\right)} g\left(x_{2}, \ldots, x_{k}\right) f\left(x_{2}, \ldots, x_{k} ; \rho, n\right) e^{-\frac{1}{2}\left\{x_{2}^{2}+a^{2}\left(x_{2}, \ldots, x_{k}\right)\right\}} d x_{2}\right]$.

$$
\prod_{i=3}^{k} \phi\left(x_{i}-\mu_{i}\right) d x_{3} \ldots d x_{k}
$$

where

$$
\begin{gathered}
f\left(x_{2}, \ldots, x_{k} ; \rho, n\right)= \\
=e^{\rho\left\{x_{2} \sin \eta+a\left(x_{2}, \ldots, x_{k}\right) \cos \eta\right\}}-e^{\rho\left\{x_{2} \cos \eta+a\left(x_{2}, \ldots, x_{k}\right) \sin \eta\right\}} .
\end{gathered}
$$

Since $a\left(x_{2}, \ldots x_{k}\right)>x_{2}$ for $x_{2}<x_{0}\left(x_{3}, \ldots, x_{k}\right)$ and $\cos \eta>\sin \eta$ for $0 \leq n<\pi / 4$, we find

$$
\begin{aligned}
& x_{2} \sin n+a\left(x_{2}, \ldots, x_{k}\right) \cos n-x_{2} \cos n-a\left(x_{2}, \ldots, x_{k}\right) \sin n= \\
& =\left\{a\left(x_{2}, \ldots, x_{k}\right)-x_{2}\right\}\{\cos n-\sin n\}>0
\end{aligned}
$$

on the region of integration. As by assumption $g\left(x_{2}, \ldots, x_{k}\right)<0$ on the region of integration and $g$ cannot be identically zero a.e. because $x_{2}^{2}+a^{2}\left(x_{2}, \ldots, x_{k}\right)$ tends to infinity for $x_{2} \rightarrow-\infty$, it follows that $\frac{\partial}{\partial n} R_{\delta}^{*}$ is negative on $0 \leq n<\pi / 4$ for all $\rho>0$. Since $R_{\delta}^{*}$ is symmetric about $n=\pi / 4$, it can only have absolute maxima for $n=0$ and $n=\frac{1}{2} \pi$, i.e. for $\mu_{1}=0$ and for $\mu_{2}=0$.

As the test $\delta$ is symmetric, its shortcoming is also symmetric in $\mu_{1}, \mu_{2}, \ldots, \mu_{k}$, implying more generally that if the shortcoming of $\delta$ reaches a maximum for a certain $\mu=\left(\mu_{1}, \ldots, \mu_{k}\right)$, at least one of any pair of its coordinates is zero.Therefore a maximum of $\mathrm{R}_{\delta}$ can only be reached for parameter points $\mu$ with $k-1$ coordinates equal to zero, i.e. for $\mu \epsilon \Omega_{\text {restr }}$.

Q.E.D. 


\section{Lemma $2 \cdot 3 \cdot 3$}

Let $k=2$ and let $\delta$ be an admissible symmetric test of $H$ against $K$. If the function $\mathrm{g}\left(\mathrm{x}_{2}\right)$ defined by (2.3.9) changes sign exactly once in the order $(-,+)$ for increasing $x_{2}$ on the interval $\left(-\infty, x_{0}\right)$, then the maximum shortcoming of $\delta$ can attain its absolute maxima only on the three half-lines $\mu_{1}=0, \mu_{2}>0$; $\mu_{2}=0, \mu_{1}>0$ and $\mu_{1}=\mu_{2}>0$.

\section{Proof:}

We start again from (2.3.10), which in this case reduces to

$$
\begin{gathered}
\frac{\partial}{\partial \eta} R_{\delta}^{*}(\rho, n)= \\
=\frac{e^{-\frac{1}{2} \rho^{2}}}{2 \pi} \int_{-\infty}^{x_{0}} g\left(x_{2}\right) f\left(x_{2} ; \rho, n\right) e^{-\frac{1}{2}\left\{x_{2}^{2}+a^{2}\left(x_{2}\right)\right\}} d x_{2},
\end{gathered}
$$

where

$$
f\left(x_{2} ; \rho, n\right)=e^{\rho\left\{x_{2} \sin n+a\left(x_{2}\right) \cos n\right\}}-e^{\rho\left\{x_{2} \cos n+a\left(x_{2}\right) \sin n\right\}} .
$$

In the proof of the preceding lemma it was seen that $f\left(x_{2} ; \rho, n\right)>0$ for $-\infty<x_{2}<x_{0}$ and $0 \leq n<\pi / 4, \rho>0$. Consider the determinant

$$
\begin{aligned}
& D=\left|\begin{array}{ll}
f\left(x_{2} ; \rho, n\right) & \frac{\partial}{\partial \eta} f\left(x_{2} ; \rho, n\right) \\
\frac{\partial}{\partial x_{2}} f\left(x_{2} ; \rho, r_{1}\right) & \frac{\partial^{2}}{\partial x_{2} \partial \eta} f\left(x_{2} ; \rho, n\right)
\end{array}\right|= \\
& =e^{\rho\left(a\left(x_{2}\right)+x_{2}\right)(\cos n+\sin n)} \cdot\left\{\rho^{2}\left(\cos ^{2} n-\sin ^{2} n\right)\left(a\left(x_{2}\right)-x_{2}\right)\left(a^{\prime}\left(x_{2}\right)-1\right)+\right. \\
& \left.2 \rho\left(a\left(x_{2}\right) \cos \eta+x_{2} \sin \eta\right)-\rho(\cos \eta-\sin \eta)\left(a^{\prime}\left(x_{2}\right)+1\right)\right\}+ \\
& 2 \rho\left(a\left(x_{2}\right) \sin n+x_{2} \cos n\right) .\left\{-\rho a^{\prime}\left(x_{2}\right) \sin n+\rho \cos n\right\}+ \\
& +e^{2} \quad .\left\{\rho a^{\prime}\left(x_{2}\right) \cos \eta-\rho \sin \eta\right\} \text {. }
\end{aligned}
$$

Let us denote the sum of the last two terms in this expression by $D^{*}$ and consider the inequality

$$
u e^{y}-v e^{-y}>(u-v)+(u+v) y,
$$

holding for $u \geq v, u+v \geq 0$ and $y>0$. Application of this inequality yields 


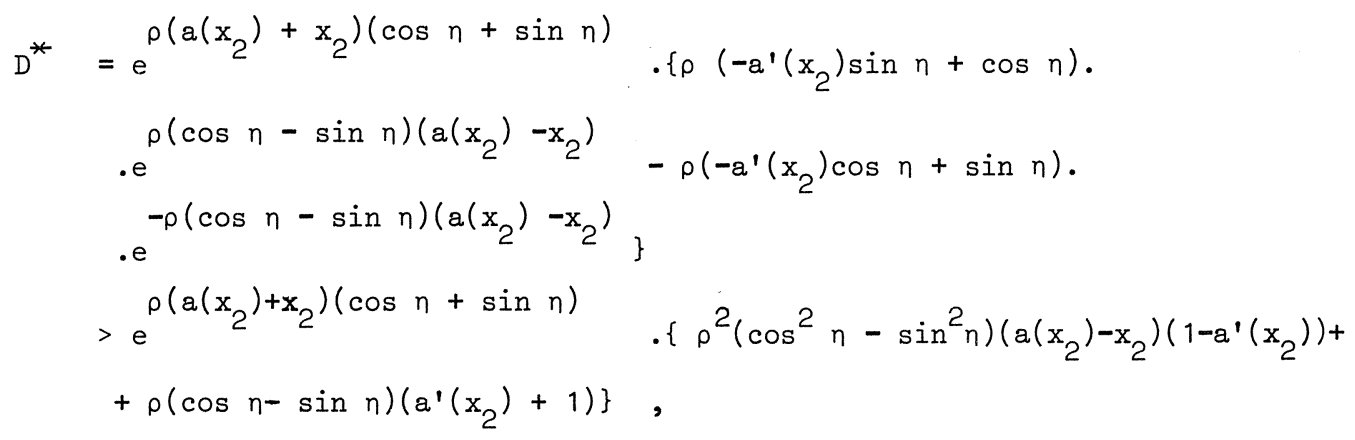

since $a^{\prime}\left(x_{0}\right)=-1$ because of the symmetry of $a$ and hence $-1 \leq a^{\prime}\left(x_{2}\right) \leq 0$ and $a\left(x_{2}\right)-x_{2}>0$ for $x_{2}<x_{0}$. It follows that $D>0$, and hence that the function $f\left(x_{2} ; \rho, n\right)$ is $\operatorname{ETP}_{2}$ in $n$ and $x_{2}$ (cf.section 1.5) for $0 \leq n<\pi / 4$ and $x_{2}<x_{0}$.

Let $g\left(x_{2}\right)$ change sign exactly once in the order $(-,+)$ for increasing $x_{2}$ on $\left(-\infty, x_{0}\right)$. Then, according to lemma 1.5.3, expression (2.3.11) and the $\operatorname{ETP}_{2}$ property of $f\left(x_{2} ; \rho, n\right)$ imply that for any $\rho>0$ the function $\partial R_{\delta}^{*}(\rho, n) / \partial n$ has at most one zero for $0 \leq n<\pi / 4$; if it does have a zero it changes sign in the order $(-,+)$ for increasing $n$. Hence for every $\rho>0$ the function $R_{\delta}^{*}(\rho, n)$ has at most one minimum and no maximum for $0<n<\pi / 4$. As $R_{\delta}^{*}$ is symmetric about $n=\pi / 4$, its absolute maxima can only occur for $n=0$ and $n=\frac{1}{2} \pi$ and $n=\pi / 4$, which proves the desired result. $\quad$ Q.E.D.

With the aid of the lemmata 2.3.2 and 2.3.3 we prove the following theorem on exponential combination procedures involving only two variables $\underline{x}_{1}$ and $\underline{x}_{2}$.

\section{Theorem 2.3.2}

Let $\mathrm{k}=2$. For any exponential combination procedure with parameters $r$ and $\mathrm{c}$ the shortcoming can assume its absolute maximum only on the three half-lines $\mu_{1}=0$, $\mu_{2}>0 ; \mu_{2}=0, \mu_{1}>0$ and $\mu_{1}=\mu_{2}>0$. Moreover, if $c \leq 2 e$, this absolute maximum can only be attained on the first two half-lines.

\section{Proof:}

The assertions of the theorem are immediate consequences of the preceding two lemmata if for exponential combination procedures the function $g\left(x_{2}\right)$ defined by (2.3.9) has at most one change of sign (in the order $(-,+)$ for increasing $x_{2}$ ) for $\mathrm{x}_{2}<\mathrm{x}_{0}$ and is nonpositive for $\mathrm{x}_{2}<\mathrm{x}_{0}$ if $\mathrm{c} \leq 2 \mathrm{e}$. We have 
$a\left(x_{2}\right)=r^{-1} \log \left(c-e^{r x_{2}}\right)$,

$g\left(x_{2}\right)=x_{2}+a\left(x_{2}\right) a^{\prime}\left(x_{2}\right)=x_{2}-r^{-1} e^{r x_{2}}\left(c-e^{r x_{2}}\right)^{-1} \log \left(c-e^{r x_{2}}\right)$.

The point $x_{0}$ for which $a\left(x_{0}\right)=x_{0}$ is given ${ }_{r} x_{2} x_{0}=r^{-1} \log (c / 2)$. To study the sign changes of $g$ on $\left(-\infty, x_{0}\right)$ we set $s=e^{x_{2}}$ and consider the function

$$
h(s)=r\left(c-e^{r x_{2}}\right) g\left(x_{2}\right)=(c-s) \log s-s \log (c-s)
$$

for $0<s<e^{r x_{0}}=\frac{1}{2} c$. We find

$$
\begin{aligned}
& \lim _{s \rightarrow 0} h(s)=-\infty, h(c / 2)=0, \\
& h^{\prime}(s)=-\log s-\log (c-s)+(c-s) / s+s /(c-s), \\
& \lim _{s \rightarrow 0} h^{\prime}(s)=+\infty, h^{\prime}(c / 2)=2(1-\log (c / 2)), \\
& h^{\prime}(s)=\{1 /(c-s)-1 / s\}+c\left\{1 /(c-s)^{2}-1 / s^{2}\right\}<0
\end{aligned}
$$

for $0<s<\frac{1}{2} c$.

If $c \leq 2 \mathrm{e}$, then $h^{\prime}(c / 2) \geq 0$ and since $h^{\prime}$ is decreasing, it is positive on $\left(0, \frac{1}{2} c\right)$. Hence $h$ is negative on $\left(0, \frac{1}{2} c\right)$, implying that $g$ is negative on $\left(-\infty, x_{0}\right)$.

If $c>2 \mathrm{e}$, then $h^{\prime}(c / 2)<0$ and since $h^{\prime}$ is decreasing, it changes sign exactly once on $\left(0, \frac{1}{2} c\right)$ in the order $(+,-)$ for increasing $s$. Hence $h$ has one maximum and no minimum on $\left(0, \frac{1}{2} \mathrm{c}\right)$. It folluws that $\mathrm{h}$ changes sign exactly once on $\left(0, \frac{1}{2} c\right)$ in the order $(-,+)$ for increasing $s$, and so does $g$ on $\left(-\infty, x_{0}\right)$ for increasing $\mathrm{x}_{2}$.

Q.E.D.

Combining theorems 2.3 .1 and 2.3 .2 we obtain

Corollary 2.3.2

For $\mathrm{k}=2$ and any size $0<\alpha<1$ the exponential combination procedure (2.3.4) of theorem 2.3.1 is MS if and only if one of the following conditions is satisfied

(i) $c(\alpha) \leq 2$ e,

(ii) the maximum shortcoming of the test on the half-line $\mu_{1}=\mu_{2}>0$ does not exceed that on the half-line $\mu_{1}=0, \mu_{2}>0$. 
The generalization of corollary 2.3.2 to $\mathrm{k}>2$ is still an open problem. It is easily verified that for general $k$ condition ( $i$ ) of the corollary again guarantees the MS character of the test (2.3.4), but this condition is of little practical interest as will be seen in the sequel. To obtain a generalization to $\mathrm{k}>2$ of condition ( $i$ ) we need a lemma similar to lemma 2.3.3 for $\mathrm{k}>2$. However, the proof of this lemma is not easily adapted to the case $k>2$, since the decreasing-increasing character of a function does not necessarily remain unperturbed if this function is integrated with respect to another variable. Nevertheless we make the following conjecture.

\section{Conjecture.}

For $\mathrm{k} \geq 2$ and any exponential combination procedure with parameters $r$ and $c$ the shortcoming can assume its absolute maximum only on the $2^{k}-1$ half-lines

$$
\mu_{i_{1}}=\ldots=\mu_{i_{m}}=0, \mu_{i_{m+1}}=\ldots=\mu_{i_{k}}>0, m=0,1, \ldots, k-1 \text {, }
$$

where $\left\{i_{1}, \ldots, i_{k}\right\}$ runs through the permutations of $\{1,2, \ldots, k\}$.

If this conjecture holds true, then for general $\mathrm{k}$ the exponential procedure (2.3.4) of theorem 2.3.1 is MS if and only if either $c(\alpha) \leq 2$ e or the maximum shortcoming of the test on the half-line $\mu_{1}=\ldots=\mu_{k-1}=0, \mu_{k}>0$ is not exceeded by that on any other of the half-lines (2.3.13).

Corollary 2.3.2 cannot easily be extended to variables. with one-parameter exponential family distributions, since the proof of the lemmata 2.3.2 and 2.3.3 leans heavily on the fact that in the normal case the envelope power is constant on hyperspheres, a property that is not shared by many other distributions.

Returning to the combination of two normal random variables, corollary 2.3.2 enables us to verify whether the exponential combination procedures of theorem 2.3.1 are MS tests of $\mathrm{H}$ against $\mathrm{K}$ ( for $\mathrm{k}=2$ ). Some details on the computations are given in section 2.5 .

It turns out that condition $(i)$ is of little practical interest, since it only covers large values of $\alpha$. For $\alpha>0.75$ the acceptance region of any exponential procedure cannot include the origin as an interior point, since it would then strictly contain the set $\left\{\left(\mathrm{x}_{1}, \mathrm{x}_{2}\right) \mid \mathrm{x}_{1} \leq 0, \mathrm{x}_{2} \leq 0\right\}$ that has probability 0.25 under H. Therefore $c(\alpha)<2$ for $\alpha>0.75$, as may be seen by substitution of 
$\left(x_{1}, x_{2}\right)=(0,0)$ in the test statistic, and hence the procedure (2.3.4) is MS. of course the estimate involved is rather rough and it turns out that the procedure of theorem 2.3.1 has $c(\alpha)=2$ for $\alpha \simeq 0.60$ and reaches the point where $c(\alpha)=2 \mathrm{e}$ only for $\alpha \simeq 0.24$. Here we have assumed without proof that $c(\alpha)$ is a decreasing function of $\alpha$.

For smaller values of $\alpha$ the validity of condition ( $i$ ) seems to end and we have to rely on condition (ii). For $\alpha=0.1$ and $\alpha=0.05$ the procedures (2.3.4) still satisfy condition (ii) and we find that the tests rejecting $H$ if

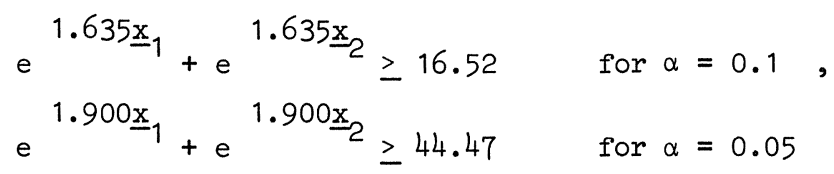

are MS for testing $H$ against $K$.

The point where the shortcoming of the test (2.3.4) assumes equal maxima on the half-lines $\mu_{1}=0, \mu_{2}>0$ and $\mu_{1}=\mu_{2}>0$ is reached for $\alpha=\alpha_{0} \simeq 0.043$. Although such a result seems difficult to prove, numerical evidence strongly suggests that exponential combination (with appropriate parameters) is MS for all $\alpha>\alpha_{0} \simeq 0.043$.

Another unsolved problem is connected with the tail probabilities of the procedures of theorem 2.3.1. Such tail probabilities can only be defined if the critical regions of these tests constitute a decreasing sequence of sets for decreasing $\alpha$. As the MS tests have different test statistics for varying $\alpha$, this property is not obvious in this case. It is easily verified that a sufficient (although not a necessary) condition for the monotonicity of the critical regions in the above sense is, that both $r(\alpha)$ and $\{r(\alpha)\}^{-1} \log c(\alpha)$ are decreasing functions of $\alpha$. Although numerical evidence points in that direction, we have not been able to prove the monotonicity of these functions. Since the shortcoming of an exponential procedure (2.3.4) assumes its maximum on the half-line $\mu_{2}=\mu_{3}=\ldots=\mu_{k}=0, \mu_{1}>0$, for $\mu_{1}=r(\alpha)$, it follows by lemma 2.2.1 that $r(\alpha)>u_{\alpha}$, giving some support to our conjecture about $r(\alpha)$.

Now let us consider sizes a smaller than $\alpha_{0}($ for $k=2)$. The situation becomes more complicated in this case. We conjecture that for values of $\alpha$ slightly smaller than $\alpha_{0}$ the LF prior distribution will assign positive probability to three points : $\left(r^{(1)}(\alpha), 0\right),\left(0, r^{(1)}(\alpha)\right)$ and $\left(r^{(2)}(\alpha), r^{(2)}(\alpha)\right)$ in the $\left(\mu_{1}, \mu_{2}\right)$-plane. The symmetry of the MS procedure implies that the first two points 
will have equal prior probabilities and hence the MS test would reject $H$ if $(2.3 .16)$

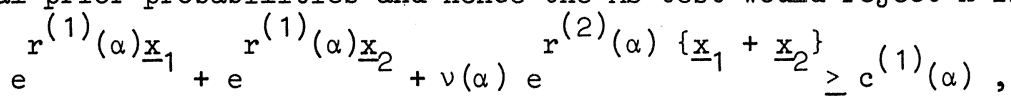

where $\nu(\alpha), r^{(1)}(\alpha), r^{(2)}(\alpha)$ and $c^{(1)}(\alpha)$ are positive and depend on $\alpha$. These four numbers are uniquely determined by the following conditions: the test (2.3.16) has size $\alpha$ and its shortcoming assumes equal maxima on the three halflines $\mu_{2}=0, \mu_{1}>0 ; \mu_{1}=0, \mu_{2}>0$ and $\mu_{1}=\mu_{2}>0$ at the three points with positive prior probabilities. Then one still has to verify whether the test is MS relative to the entire parameter space. Since the computational labour involved is enormous and the test statistic (2.3.16) is too complicated to have any practical value, we did not start a further investigation.

As a further decreases towards zero, the LF prior distribution will supposedly concentrate on an indefinitely increasing (but finite) number of points (cf. corollary 2.3.1). This will be demonstrated in the next section. As a result the number of terms involved in the test statistic of the MS test also increases indefinitely for $\alpha \rightarrow 0$ and the task of determining the MS test becomes even more hopeless.

We briefly consider the case $\mathrm{k}=3$. Although not so simple as the case of two variables, it is still fairly easy to determine the parameters $r(\alpha)$ and $c(\alpha)$ of the test (2.3.4) of theorem 2.3.1. If the conjecture about the possible maxima of the shortcoming of exponential combination procedures holds true, it can be verified whether this test is MS for testing $H$ against $K$ by computing the maximum shortcoming on the two half-lines $\mu_{1}=\mu_{2}=\mu_{3}>0$ and $\mu_{1}=0$, $\mu_{2}=\mu_{3}>0$ and comparing these with $R_{\delta_{\alpha}}(r(\alpha), 0,0)$. These computations were performed for $\alpha=0.1$ and $\alpha=0.05$. It turned out that the test that rejects $H$ if

$$
e^{1.706 \underline{x}_{1}}+e^{1.706 \underline{x}_{2}}+e^{1.706 \underline{x}_{3}} \geq 27.35 \text { for } \alpha=0.1
$$

has a larger maximum shortcoming on the half-line $\mu_{2}=\mu_{3}=0, \mu_{1}>0$ than on both other half-lines and hence this test is supposedly the size - 0.1 MS test. For $\alpha=0.05$ however, exponential combination cannot be MS since the test (2.3.4) has a larger maximum shortcoming on the half-line $\mu_{1}=0, \mu_{2}=\mu_{3}>0$ than on the half-line $\mu_{2}=\mu_{3}=0, \mu_{1}>0$. 
For $\mathrm{k}>3$ the complexity of the computations necessary to determine the parameters $r(\alpha)$ and $c(\alpha)$ of the test of theorem 2.3.1 increases at a high rate. For $k=4$ some partial results were obtained by SCHAAFSMA [46], but nevertheless we are inclined to say that the usefulness of MS tests ends at $k=3$, from theoretical as well as from practical points of view. In this connection we remark that SCHAAFSMA ([46]th.5) has shown that, for fixed $\alpha$, the maximum shortcoming of the MS test tends to $1-\alpha$ for $k \rightarrow \infty$. Hence tests with uniformly good power properties simply do not exist for large $\mathrm{k}$.

\subsection{ASYMPTOTICALLY OPTIMAL PROCEDURES FOR $\alpha \rightarrow 0$.}

In this section we study the problem of testing $H: \mu=0$ against $K$ : $\mu . \geq 0$ (cf.section 2.2) for small values of the significance level $\alpha$. In the preceding section it was shown that for $\mathrm{k}=2$ the LF prior distribution is discrete with a finite spectrum for all $\alpha(0<\alpha<1)$, cf. corollary 2.3.1. The MS tests of $\mathrm{H}$ against $\mathrm{K}$ were obtained in a number of cases and turned out to be exponential combination procedures in these cases, but we found empirically that exponential combination is not MS for small values of $\alpha$. In view of this we suggested that the LF prior distributions concentrate on an ever increasing number of points for $\alpha \rightarrow 0$. These ideas are confirmed by the following results.

Theorem 2.4.1

Let $\mathrm{k} \geq 2$, let $\mathrm{m}$ be any fixed positive integer and let $\varepsilon(0<\varepsilon<1)$ be arbitrary. Consider a set of discrete prior distributions $\{\xi(\alpha) \mid 0<\alpha<1\}$ assigning nonnegative probability to $m$ points $\mu^{(1, \alpha)}, \mu^{(2, \alpha)}, \ldots, \mu(m, \alpha)$ in the set $\mu . \geq 0$; the position of these $m$ points and the associated prior probabilities $p_{j, \alpha}$ $(j=1,2, \ldots, m)$ may depend on $\alpha$. The half-lines in the parameter space through the origin and $\mu^{(j, \alpha)}$ are denoted by $M_{j, \alpha}(j=1,2, \ldots m)$ and the distances from the origin of the points $\mu^{(j, \alpha)}$ by $\|\mu(j, \alpha)\|=\rho_{j, \alpha}(j=1,2, \ldots m)$. Let $M_{0, \alpha}$ denote the half-line through the origin and the point $\mu^{(0, \alpha)}=\sum_{1}^{m} q_{j, \alpha}{ }^{(j, \alpha)}$, where $q_{j, \alpha}=p_{j, \alpha}$. $. \exp \left(-\frac{1}{2} \rho_{j, \alpha}^{2}\right), j=1,2, \ldots, m$. Let $R_{\alpha}$ denote the shortcoming of the size- $1_{j}$ test $\delta_{\alpha}$ of $H$ which is Bayes with respect to $\xi(\alpha)$. Then,

(i) if the condition 
$(2.4 .1)$

$$
\lim _{\alpha \rightarrow 0} u_{\alpha} \rho_{j, \alpha}=\infty \text { for } j=1,2, \ldots, m
$$

is satisfied, we have

(2.4.2) $\quad \lim _{\alpha \rightarrow 0} \sup _{\mu \in L_{\alpha}} R_{\alpha}(\mu)=1$ uniformly for all $L_{\alpha} \in \mathscr{L}{ }_{\alpha}$,

where $\mathcal{L}{ }_{\alpha}$ is the set of all half-lines $L_{\alpha}$ in the parameter space through the origin making an angle larger than arc cos $(1-\varepsilon)$ with each of the $M_{j, \alpha}$ for $j=1,2, \ldots, m$;

(ii) if on the other hand the condition

$$
\lim _{\alpha \rightarrow 0} u_{\alpha} \rho_{j, \alpha}=0 \quad \text { for } j=1,2, \ldots, m
$$

is satisfied, we have

(2.4.4) $\lim _{\alpha \rightarrow 0} \sup _{\mu \in L_{\alpha}} R_{\alpha}(\mu)=1$ uniformly for all $\mathrm{L}_{\alpha} \in \mathcal{L}_{\alpha}^{0}$, where $\mathcal{L}_{\alpha}^{0}$ is the set of all half-lines $L_{\alpha}$ in the parameter space through the origin making an angle larger than arc $\cos (1-\varepsilon)$ with $\mathrm{M}_{0, \alpha}$.

Proof:

Let $\mathrm{A}_{\alpha}$ denote the acceptance region of the test $\delta_{\alpha}$. We write

$$
\mu^{(j, \alpha)}=\left(\zeta_{1, j, \alpha}{ }^{\rho, \alpha}, \ldots ., \zeta_{k, j, \alpha}{ }^{\rho}{ }_{j, \alpha}\right), \quad j=1,2, \ldots, m,
$$

where $\rho_{j, \alpha}>0$ and $\zeta_{i, j, \alpha} \geq 0(i=1,2, \ldots, k), \sum_{i} \zeta_{i, j, \alpha}^{2}=1$, and

(2.4.5) $\quad t_{\alpha}(x)=t_{\alpha}\left(x_{1}, \ldots, x_{k}\right)=\sum_{j=1}^{m} q_{j, \alpha} \exp \left(\rho_{j, \alpha} \sum_{i=1}^{k} \zeta_{i, j, \alpha} x_{i}\right)$.

Then we have by the NEYMAN-PEARSON lemma

$$
A_{\alpha}=\left\{x \mid t_{\alpha}(x)<c_{\alpha}\right\},
$$

where $c_{\alpha}$ is an appropriate constant.

First we consider case (i). Let $\varepsilon_{1}, 0<\varepsilon_{1}<1$, be arbitrary. Let $L_{\alpha} \in \mathcal{L}_{\alpha}$ and let $\psi_{j, \alpha}$ denote the acute angle between $L_{\alpha}$ and $M_{j, \alpha}$. Then, by assumption, $\cos \psi_{j, \alpha}<1-\varepsilon$ for $j=1,2, \ldots, m$. Consider the map $\mu \rightarrow x=\mu$ of $\mu$-space onto $x$-space. By this map there corresponds to each line (or point) in $\mu$-space a line (or point) in $\mathrm{x}$-space. Lines in $\mathrm{x}$-space corresponding to lines in $\mu$-space are denoted by * superscripts in this proof. Let $\mu\left(d ; L_{\alpha}\right)$ be the point on $L_{\alpha}$ at a distance $d$ from the origin 0 ; the corresponding point in $x$-space is denoted by $x\left(d ; L_{\alpha}^{*}\right)$. At $\underline{x}=x\left(d ; L_{\alpha}^{*}\right)$ the test statistic $t_{\alpha}(\underline{x})$ assumes the value 


$$
t_{\alpha}\left(x\left(d ; L_{\alpha}^{*}\right)\right)=\sum_{j=1}^{m} q_{j, \alpha} \exp \left(d \rho_{j, \alpha} \cos \psi_{j, \alpha}\right) .
$$

The point of intersection of the half-line $M_{g, \alpha}^{*}$ and the hypersphere with radius $u_{\alpha}$ and centre $O$ in $x$-space is an interior or boundary point of $A_{\alpha}$, for otherwise there would exist a supporting hyperplane of $A_{\alpha}$ at a distance smaller than $u_{\alpha}$ from the origin and the size of $\delta_{\alpha}$ would be larger than $\alpha$. Hence

$$
\sum_{j=1}^{m} q_{j, \alpha} \exp \left(\rho_{j, \alpha} u_{\alpha} \sum_{i=1}^{k} \zeta_{i, j, \alpha} \zeta_{i, g, \alpha}\right) \leq c_{\alpha}
$$

for $g=1,2, \ldots, m$, implying

$$
\frac{1}{m} \sum_{j=1}^{m} q_{j, \alpha} \sum_{g=1}^{m} \exp \left(\rho_{j, \alpha} u_{\alpha} \sum_{i=1}^{k} \zeta_{i, j, \alpha} \zeta_{i, g, \alpha}\right) \leq c_{\alpha} .
$$

It follows from this inequality and (2.4.6) that

$$
t_{\alpha}\left(x\left(d ; L_{\alpha}^{*}\right)\right)<c_{\alpha}
$$

if the conditions

$(2.4 \cdot 7)$

$$
\begin{aligned}
& \exp \left(\alpha_{j, \alpha} \cos \psi_{j, \alpha}\right)< \\
< & \left.\frac{1}{m}\left\{\exp \left(\rho_{j, \alpha} u\right)_{\alpha}\right)+\sum_{\substack{g=1 \\
g \neq j}}^{m} \exp \left(\rho_{j, \alpha} u_{\alpha_{i=1}} \sum_{i=1}^{k} \zeta_{i, j, \alpha} \zeta_{i, g, \alpha}\right)\right\}
\end{aligned}
$$

are satisfied for $j=1,2, \ldots, m$.

Put $\mathrm{b}=(2 \log \mathrm{m}) / \varepsilon$; then there exists a positive number $\alpha_{0}(\varepsilon)$ such that $\rho_{j, \alpha} u_{\alpha} \geq b$ for $j=1,2, \ldots, m$ and all $\alpha<\alpha_{0}(\varepsilon)$ by condition (2.4.1). Choose

$$
\mathrm{d}_{\alpha}=\left(1+\frac{1}{2} \varepsilon\right) \mathrm{u}_{\alpha}
$$

then

$$
\begin{aligned}
d_{\alpha} \rho_{j, \alpha} \cos \psi_{j, \alpha} & =\left(1+\frac{1}{2} \varepsilon\right) u_{\alpha} \rho_{j, \alpha} \cos \psi_{j, \alpha}<\left(1+\frac{1}{2} \varepsilon\right) u_{\alpha} \rho_{j, \alpha}(1-\varepsilon) \\
& \leq \rho_{j, \alpha} u_{\alpha}-(1+\varepsilon) \log m \\
& <\rho_{j, \alpha} u_{\alpha}-\log m
\end{aligned}
$$


implying

$$
\exp \left(d_{\alpha} \rho_{j, \alpha} \cos \psi_{j, \alpha}\right)<\frac{1}{m} \exp \left(\rho_{j, \alpha} u_{\alpha}\right), j=1,2, \ldots, m .
$$

Hence, if $d=d_{\alpha}$, the inequalities (2.4.7) are satisfied and therefore $x\left(d_{\alpha} ; L_{\alpha}^{*}\right)$ is an interior point of $A_{\alpha}$.

Now consider a simple alternative

$$
\mu^{(\alpha)}=\mu\left(\alpha_{\alpha}-\frac{1}{4} \varepsilon u_{\alpha} ; L_{\alpha}\right)
$$

on $\mathrm{L}_{\alpha}$. The size- $\alpha$ envelope power at $\mu=\mu^{(\alpha)}$ is equal to (cf. (2.4.8))

$$
\beta_{\alpha}^{+}\left({ }^{(\alpha)}\right)=\Phi\left(\frac{1}{4} \varepsilon u_{\alpha}\right) .
$$

Let $S_{\alpha}$ be the solid hypersphere with centre $x\left(d_{\alpha}-\frac{1}{4} \varepsilon u_{\alpha} ; L_{\alpha}^{*}\right)$ and radius $\frac{1}{8} \varepsilon u_{\alpha}$ in $\mathrm{x}$-space. Then $\mathrm{S}_{\alpha} \subset \mathrm{A}_{\alpha}$, for otherwise there would again exist a supporting hyperplane of $A_{\alpha}$ at a distance smaller than $u_{\alpha}$ from the origin. Hence the power of $\delta_{\alpha}$ at $\mu=\mu(\alpha)$ satisfies the inequality

$$
\beta_{\delta_{\alpha}}(\mu(\alpha))<1-P\left(\left(\underline{x}_{1}, \ldots, \underline{x}_{k}\right) \in S_{\alpha} \mid \mu(\alpha)\right)=P\left(\underline{x}_{k}^{2}>\frac{1}{8} \varepsilon u_{\alpha}\right),
$$

where $x_{k}^{2}$ has a chi-square distribution with $k$ degrees of freedom. Thus $\beta_{\alpha}^{+}\left(\mu(\alpha) \stackrel{-, k}{\rightarrow}\right.$ and $\beta_{\delta_{\alpha}}(\mu(\alpha)) \rightarrow 0$ for $\alpha \rightarrow 0$, and we find that for all $\alpha<\alpha_{0}\left(\varepsilon, \varepsilon_{1}\right)$

$$
\mathrm{R}_{\delta_{\alpha}}\left(\mu^{(\alpha)}\right)=\beta_{\alpha}^{+}\left({ }^{(\alpha)}\right)-\beta_{\delta_{\alpha}}\left({ }^{(\alpha)}\right)>1-\varepsilon_{1},
$$

establishing the desired result (2.4.2), since $\alpha_{0}\left(\varepsilon, \varepsilon_{1}\right)$ may be taken independent of the particular choice of $\mathrm{L}_{\alpha}$.

To prove the second part of the theorem, consider an arbitrary half-line $\mathrm{L}_{\alpha} \in \mathcal{L}_{\alpha}^{0}$ with direction cosines $\sigma_{1, \alpha}, \ldots, \sigma_{\mathrm{k}, \alpha}\left(\sigma_{i, \alpha} \geq 0\right.$ for $\left.\mathrm{i}=1,2, \ldots, \mathrm{k}\right)$. Let $\tau_{1, \alpha}, \ldots, \tau_{k, \alpha}$ denote the direction cosines of the half-line $\mathrm{M}_{0, \alpha}$ :

$$
\left.\tau_{i, \alpha}=\left(\sum_{j=1}^{m} q_{j, \alpha} \alpha_{j, \alpha}{ }^{\zeta}, j, \alpha\right) \sum_{i^{\prime}=1}^{k}\left(\sum_{g=1}^{m} q_{g, \alpha} \rho_{g, \alpha^{\zeta}} i_{, g, \alpha}\right)^{2}\right\}^{-\frac{1}{2}}
$$

for $i=1,2, \ldots, m$. The point of intersection of $M_{0, \alpha}^{*}$ and the hypersphere with radius $u_{\alpha}$ and centre 0 in $x$-space is an interior or boundary point of $A_{\alpha}$ (by the by now familiar argument), and hence 
$(2.4 .9)$

$$
\sum_{j=1}^{m} q_{j, \alpha} \exp \left(\rho_{j, \alpha} u_{\alpha} \sum_{i=1}^{k} \zeta_{i, j, \alpha}{ }^{\tau}{ }_{i, \alpha}\right) \leq c_{\alpha} .
$$

By condition (2.4.3) $\rho_{j, \alpha} u_{\alpha}<\frac{1}{4} \varepsilon$ for $j=1,2, \ldots, m$ and all $\alpha<\alpha_{0}(\varepsilon)$. Hence, for $\alpha<\alpha_{0}(\varepsilon)$ and $\alpha_{\alpha}$ defined by (2.4.8),

$$
\begin{aligned}
& t\left(x\left(d_{\alpha} ; L_{\alpha}^{*}\right)\right)=\sum_{j=1}^{m} q_{j, \alpha} \exp \left(\left(1+\frac{1}{2} \varepsilon\right) u_{\alpha} \rho_{j, \alpha} \sum_{i=1}^{k} \zeta_{i, j, \alpha} \sigma_{i, \alpha}\right)< \\
& <\sum_{j=1}^{m} q_{j, \alpha}\left[1+\left(1+\frac{1}{2} \varepsilon\right) u_{\alpha} \rho_{j, \alpha} \sum_{i=1}^{k} \zeta_{i, j, \alpha} \sigma_{i, \alpha}+\frac{1}{5} \varepsilon\left(1+\frac{1}{2} \varepsilon\right)^{2} u_{\alpha} \rho j, \alpha \sum_{i=1}^{k} \zeta_{i, j, \alpha} \sigma_{i, \alpha}\right] \\
& <\sum_{j=1}^{m} q_{j, \alpha}\left[1+(1+\varepsilon) u_{\alpha} \rho_{j, \alpha} \sum_{i=1}^{k} \zeta_{i, j, \alpha} \sigma_{i, \alpha}\right] \\
& =\sum_{j=1}^{m} q_{j, \alpha}+(1+\varepsilon) u_{\alpha} \sum_{i=1}^{k} \sigma_{i, \alpha} \sum_{j=1}^{m} q_{j, \alpha} \rho_{j, \alpha}{ }_{j, j, \alpha} \\
& =\sum_{j=1}^{m} q_{j, \alpha}+(1+\varepsilon) u_{\alpha} \sum_{i=1}^{k} \sigma_{i, \alpha} \tau_{i, \alpha}\left\{\sum_{i^{\prime}=1}^{k}\left(\sum_{g=1}^{m} q_{g, c^{\prime}} \rho_{g, \alpha} \zeta_{i^{\prime}, g, \alpha}\right)^{2}\right\}^{\frac{1}{2}} \\
& <\sum_{j=1}^{m} q_{j, \alpha}+(1+\varepsilon)(1-\varepsilon) u_{\alpha}\left\{\sum_{i=1}^{k}\left(\sum_{g=1}^{m} q_{g, \alpha} \rho_{g, \alpha} \zeta i, g, \alpha\right)^{2}\right\}{ }^{\frac{1}{2}} \\
& <\sum_{j=1}^{m} q_{j, \alpha}\left[1+u_{\alpha} \rho_{j, \alpha} \sum_{i=1}^{k} \zeta_{i, j, \alpha} \tau_{i, \alpha}\right] \\
& <\sum_{j=1}^{m} q_{j, \alpha} \exp \left(u_{\alpha} \rho_{j, \alpha} \sum_{i=1}^{k} \zeta_{i, j, \alpha} \tau_{i, \alpha}\right) \leq c_{\alpha},
\end{aligned}
$$

where the third inequality is motivated by the fact that by assumption

$$
\sum \sigma_{i, \alpha}{ }_{i, \alpha}<1-\varepsilon \text {. Hence } x\left(d_{\alpha} ; L_{\alpha}^{*}\right) \text { is an interior point of } A_{\alpha} \text { and the proof of }
$$

(2.4.4) can be completed by the same argument that we used in the first part of the proof.

Q.E.D.

The proof of the theorem above is based on the fact that, roughly speaking, for $\alpha \rightarrow 0$ under condition (2.4.1) the acceptance region of $\delta_{\alpha}$ approaches regions bounded by $m$ hyperplanes orthogonal to $\mathrm{M}_{j, \alpha}^{*}$ and that for $\alpha \rightarrow 0$ under condition (2.4.3) 
the acceptance region of $\delta_{\alpha}$ approaches regions bounded by one hyperplane orthogonal to $\mathrm{M}_{0, \alpha}^{*}$.

The exponential combination procedures of theorem 2.3.1 are Bayes with respect to prior distributions which are concentrated on $\mathrm{k}$ points in the parameter space. By lemma 2.2.1 these prior distributions satisfy condition (2.4.1) of theorem 2.4.1 and hence the maximum shortcoming of exponential combination procedures tends to one for $\alpha \rightarrow 0$ and all fixed $k \geq 2$. In the sequel we shall find that the maximum shortcoming of the likelihood ratio test of $\mathrm{H}$ against $\mathrm{K}$ tends to zero for $\alpha \rightarrow 0$ (lemma 2.4.2), implying that exponential combination cannot be MS for small $\alpha$. Thus we obtain as a first application of theorem 2.4.1

\section{Corollary 2.4 .1}

For each $k \geq 2$ there exists a positive number $\alpha_{0}(k)$ such that exponential combination procedures cannot be MS tests of $\mathrm{H}$ against $\mathrm{K}$ for any $\alpha<\alpha_{0}(\mathrm{k})$. Moreover, the maximum shortcoming of the exponential combination procedures of theorem 2.3.1 tends to one for $\alpha \rightarrow 0$ on any half-line bounded away from $\Omega_{\text {restr }}$ (cf.(2.3.3)).

The LF prior distribution assigns probability one to the set of points for which the shortcoming of the corresponding MS test attains its absolute maximum. If for $\alpha \rightarrow 0$ the LF prior distributions would remain concentrated on a bounded number of points, condition (2.4.1) of theorem 2.4.1 is satisfied by lemma 2.2.1 and hence the maximum shortcoming of the MS tests would tend to one for $\alpha \rightarrow 0$. However, this is impossible because the maximum shortcoming of the LR tests tends to zero for $\alpha \rightarrow 0$. Hence we obtain as a second application of theorem 2.4.1

\section{Corollary 2.4 .2}

For $\alpha \rightarrow 0$ the LF prior distributions do not remain concentrated on a bounded number of points.

As the MS tests have a very complicated structure for small values of $\alpha$, Wh try to find tests with uniformly good power properties by another approach.

To this end we consider the likelihood ratio (LR) test of the hypothesis $H$ against $K$. It is easily verified that the size- $\alpha$ LR test rejects $H$ if

$$
\sum_{i=1}^{k} \underline{x}_{i}^{2} I_{(0, \infty)}\left(\underline{x}_{i}\right) \geq \rho_{k, \alpha}^{2},
$$


where $I_{(0, \infty)}$ denotes the characteristic function of the set $(0, \infty)$ and $\rho_{k, \alpha}$ is an appropriate constant. We note that LR tests exist only for sizes $\alpha$ smaller than $1-2^{-k}$, since the acceptance region $A_{L R, \alpha}$ of the test strictly contains the negative orthant. In the positive orthant the region $A_{L R, \alpha}$ is bounded by a hypersphere $\sum x_{i}^{2}=\rho_{k, \alpha}^{2}$. It follows from lemma 1.4 .6 that the LR tests are admissible; however, by theorem 2.2.1 these tests are not Bayes and hence cannot be MS for any size $\alpha$.

As a tends to zero, $\rho_{k, \alpha}$ tends to infinity. Moreover,

$$
\rho_{\mathrm{k}, \alpha}>\mathrm{u}_{\alpha},
$$

since otherwise there would exist a supporting hyperplane of $A_{L R, \alpha}$ at a distance of at most $u_{\alpha}$ from the origin and the LR test would have a larger size than $\alpha$. In the following lemma the asymptotic behaviour of $\rho_{k, \alpha}$ is described more precisely in terms of $u_{\alpha}$.

Lemma 2.4 .1

For $\alpha \rightarrow 0$

$$
\rho_{k, \alpha}=u_{\alpha}+(k-1) u_{\alpha}^{-1} \log u_{\alpha}+\left\{\frac{1}{2} \log \pi-\frac{3}{2}(k-1) \log 2-\log \Gamma\left(\frac{k}{2}\right)\right\} u_{\alpha}^{-1}+\sigma\left(u_{\alpha}^{-2}\right) .
$$

Proof:

During the proof we omit the index $k$ of $\rho_{k, \alpha}$. Let $t_{L R}$ denote the test statistic of the LR test appearing in (2.4.10). Then under $H$

$$
\begin{aligned}
P\left(\underline{t}_{L R}<\rho_{\alpha}^{2}\right) \quad= & \sum_{j=0}^{k}\left(\begin{array}{l}
k \\
j
\end{array}\right) P\left(\underline{t}_{L R}<\rho_{\alpha}^{2} \mid \underline{x}_{1}>0, \ldots, \underline{x}_{j}>0, \underline{x}_{j+1} \leq 0, \ldots, \underline{x}_{k} \leq 0\right) . \\
& . P\left(\underline{x}_{1}>0, \ldots, \underline{x}_{j}>0, \underline{x}_{j+1} \leq 0, \ldots, \underline{x}_{k} \leq 0\right) \\
= & 2^{-k} \sum_{j=0}^{k}\left(\begin{array}{l}
k \\
j
\end{array}\right) P\left(\sum_{i=1}^{j} \underline{x}_{i}^{2}<\rho_{\alpha}^{2}\right) \\
= & 2^{-k}\left[1+\sum_{j=1}^{k}\left(\begin{array}{l}
k \\
j
\end{array}\right)\left\{2^{\frac{1}{2} j} \Gamma\left(\frac{j}{2}\right)\right\}^{-1} \int_{0}^{\rho_{\alpha}^{2}} w^{\frac{1}{2} j-1} e^{-\frac{1}{2} w} d w\right],
\end{aligned}
$$

where, by repeated partial integration, 
$(2.4 .13)$

$$
\begin{aligned}
\left\{2^{\frac{1}{2} j} \Gamma \quad\right. & \left.\left(\frac{j}{2}\right)\right\}^{-1} \int_{0}^{\rho_{\alpha}^{2}} w^{\frac{1}{2} j-1} e^{-\frac{1}{2} w} d w= \\
& =\left\{\begin{array}{l}
1-\exp \left(-\frac{1}{2} \rho_{\alpha}^{2}\right) \sum_{i=0}^{\frac{1}{2} j-1}\left(\frac{1}{2} \rho_{\alpha}^{2}\right)^{i} / \Gamma(i+1) \text { if } j \text { is even } \\
2 \Phi\left(\rho_{\alpha}\right)-1-\exp \left(-\frac{1}{2} \rho_{\alpha}^{2}\right) \sum_{i=1}^{\frac{1}{2}(j-1)}\left(\frac{1}{2} \rho_{\alpha}^{2}\right)^{i-\frac{1}{2}} / \Gamma\left(i+\frac{1}{2}\right) \text { if } j \text { is odd. }
\end{array}\right.
\end{aligned}
$$

The numbers $\rho_{\alpha}$ and $u_{\alpha}$ are connected by the relation

$$
\Phi\left(u_{\alpha}\right)=P\left(\underline{t}_{L R}<\rho_{\alpha}^{2} \mid H\right)=1-\alpha .
$$

Since both $u_{\alpha}$ and $\rho_{\alpha}$ tend to infinity for $\alpha \rightarrow 0$, the well-known approximation

$$
\Phi(z)=1-\exp \left(-\frac{1}{2} z^{2}\right)\left\{\frac{1}{z \sqrt{2 \pi}}+\sigma\left(z^{-3}\right)\right\} \text { for } z \rightarrow \infty
$$

can be applied to both $\Phi\left(u_{\alpha}\right)$ and $\Phi\left(\rho_{\alpha}\right)$. Substituting (2.4.13) into (2.4.12) and applying (2.4.15) we find, for $\alpha \rightarrow 0$,

$$
P\left(\underline{t}_{L R}<\rho_{\alpha}^{2} \mid H\right)=1-\frac{2^{-k}}{\Gamma\left(\frac{k}{2}\right)} e^{-\frac{1}{2} \rho_{\alpha}^{2}}\left(\frac{1}{2} \rho_{\alpha}^{2}\right)^{\frac{1}{2} k-1}\left(1+\sigma\left(\rho_{\alpha}^{-1}\right)\right) .
$$

Combining (2.4.14) through (2.4.16) we obtain

or, taking logarithms,

$$
\left\{\frac{1}{u_{\alpha} \sqrt{2 \pi}}+\sigma\left(u_{\alpha}^{-3}\right)\right\} e^{-\frac{1}{2} u_{\alpha}^{2}}=2^{-k}\left\{\frac{\rho}{\rho^{k-2}} \frac{1}{2^{\frac{1}{2} k-1} \Gamma(k / 2)}+\sigma\left(\rho_{\alpha}^{k-3}\right)\right\} e^{-\frac{1}{2} \rho_{\alpha}^{2}},
$$

$$
\begin{aligned}
& \frac{1}{2}\left(\rho_{\alpha}^{2}-u_{\alpha}^{2}\right)-\log u_{\alpha}-\frac{1}{2} \log 2 \pi+\sigma\left(u_{\alpha}^{-2}\right)=-k \log 2+(k-2) \log \rho_{\alpha}+ \\
& -\frac{1}{2}(k-2) \log 2-\log \Gamma\left(\frac{k}{2}\right)+\sigma\left(\rho_{\alpha}^{-1}\right)
\end{aligned}
$$

and hence

$$
\begin{aligned}
\rho_{\alpha}-u_{\alpha}=2\left(\rho_{\alpha}+u_{\alpha}\right)^{-1} & \left\{(k-2) \log \rho_{\alpha}+\log u_{\alpha}-\frac{3}{2}(k-1) \log 2+\right. \\
+ & \left.\frac{1}{2} \log \pi-\log \Gamma\left(\frac{k}{2}\right)+\sigma\left(u_{\alpha}^{-1}\right)\right\}
\end{aligned}
$$

for $\alpha \rightarrow 0$. This relation immediately yields (2.4.11). 
In particular we have

$(2.4 .17)$

$$
\lim _{\alpha \rightarrow 0}\left(\rho_{k, \alpha}-u_{\alpha}\right)=0
$$

From(2.4.12) and (2.4.13) critical values of the LR test can easily be determined. Table 2.4.1 shows $\rho_{k, \alpha}^{2}$ for selected values of $k$ and $\alpha$.

Let $R_{L R, \alpha}$ denote the shortcoming of the size- $\alpha$ LR test. Since the LR test satisfies the conditions of lemma 2.3.2, there exists a positive number $\mu_{\alpha}$ such that for all $\mu=\left(\mu_{1}, \ldots, \mu_{k}\right)$

$$
R_{L R, \alpha}(\mu) \leq R_{L R, \alpha}\left(\mu_{\alpha}, 0, \ldots, 0\right) .
$$

Consider therefore

\begin{tabular}{|c|c|c|c|c|c|c|c|}
\hline & 2 & 3 & 4 & 5 & 6 & 7 & 8 \\
\hline .2 & 1.725 & 2.595 & 3.390 & 4.142 & 4.865 & 5.567 & 6.252 \\
\hline .1 & 2.952 & 4.010 & 4.955 & 5.835 & 6.671 & 7.476 & 8.257 \\
\hline .05 & 4.231 & 5.434 & 6.498 & 7.480 & 8.407 & 9.295 & 10.152 \\
\hline .025 & 5.537 & 6.861 & 8.023 & 9.091 & 10.095 & 11.053 & 11.976 \\
\hline .01 & 7.289 & 8.746 & 10.019 & 11.183 & 12.274 & 13.312 & 14.310 \\
\hline .005 & 8.628 & 10.171 & 11.516 & 12.744 & 13.893 & 14.985 & 16.032 \\
\hline .001 & 11.763 & 13.474 & 14.962 & 16.317 & $17 \cdot 582$ & 18.780 & 19.927 \\
\hline .0001 & 16.287 & 18.188 & 19.840 & 21.34 & 22.74 & 24.06 & 25.33 \\
\hline .00001 & 20.84 & 22.89 & 24.67 & 26.30 & 27.81 & 29.23 & 30.59 \\
\hline
\end{tabular}

$$
R_{L R, \alpha}\left(\mu_{\alpha}, 0, \ldots, 0\right)=P\left(\underline{t}_{L R, \alpha}<\rho_{k, \alpha}^{2} \mid \mu_{\alpha}, 0, \ldots, 0\right)-\Phi\left(u_{\alpha}-\mu_{\alpha}\right)<
$$

Table 2.4.1. Critical values $\rho_{\mathrm{k}, \alpha}^{2}$ of the LR combination procedure. 


$$
\begin{aligned}
& <\mathrm{P}\left(\underline{\mathrm{x}}_{1}<\rho_{\mathrm{k}, \alpha} \mid \mu_{\alpha}\right)-\Phi\left(\mathrm{u}_{\alpha}-\mu_{\alpha}\right) \\
& =\Phi\left(\rho_{\mathrm{k}, \alpha}-\mu_{\alpha}\right)-\Phi\left(\mathrm{u}_{\alpha}-\mu_{\alpha}\right) .
\end{aligned}
$$

But (2.4.17) implies that this expression tends to zero for $\alpha \rightarrow 0$, and thus we have proved

\section{Lemma 2.4.2}

The shortcoming $R_{L R, \alpha}$ of the size- $\alpha$ LR combination procedure attains its absolute maximum exclusively on the edges of the parameter space and

$$
\lim _{\alpha \rightarrow 0} R_{L R, \alpha}(\mu)=0 \quad \text { uniformly for all } \mu \geq 0 \text {. }
$$

We have thus shown that the maximum shortcoming of LR tests tends to zero for $\alpha \rightarrow 0$. This is of course a desirable property. Families of tests with this property will be called asymptotically optimal for $\alpha \rightarrow 0$. Obviously the MS size- $\alpha$ tests have a smaller maximum shortcoming, but this advantage becomes negligible for (very) small values of $\alpha$.

It now remains to investigate what other families of combination procedures besides the LR tests are asymptotically optimal for $\alpha \rightarrow 0$. We shall show that, in a sense to be made precise below, any family of admissible and asymtotically optimal tests approaches the LR tests for $\alpha \rightarrow 0$.

Consider an arbitrary family of admissible (nonrandomized) combination procedures with acceptance regions $A_{\alpha}(0<\alpha<1)$, where the procedure characterized by $A_{\alpha}$ has size $\alpha$ and shortcoming $R_{\alpha}$. Let $A^{*}$ denote the intersection of the set $A$ and the positive orthant, i.e.

$$
A^{*}=A \cap\{x \mid x \geq 0\}
$$

and let $d(A, B)$ denote the Hausdorff distance of two sets $A$ and $B(c f .(1.4 .20))$.

Theorem 2.4.2
$(2.4 .19)$
$\lim _{\alpha \rightarrow 0} \sup _{\mu .>0} R_{\alpha}(\mu)=0$
if and only if
$(2.4 .20)$
$\lim _{\alpha \rightarrow 0} d\left(A_{\alpha}^{*}, A_{L R, \alpha}^{*}\right)=0$. 


\section{Proof:}

First we show that (2.4.19) implies (2.4.20). Suppose that (2.4.20) is false and hence a number $\delta>0$ exists such that for any $\alpha>0$ there is $\alpha^{\prime}<\alpha$ satisfying $(2.4 .21)$

$$
\mathrm{d}\left(\mathrm{A}_{\alpha}^{*}, \mathrm{~A}_{\mathrm{LR}, \alpha}^{*}\right)>\delta \text {. }
$$

Let $\varepsilon$ be defined by

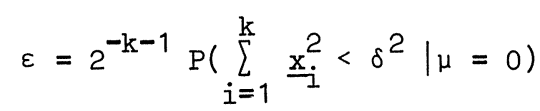

and let $q>\delta$ be so large that

$$
P\left(\sum_{i=1}^{k} \underline{x}_{i}^{2} \geq q^{2} \mid \mu=0\right)<\varepsilon .
$$

Furthermore let $\alpha_{0}$ be so small that for all $\alpha<\alpha_{0}$ we have

(i) $\rho_{k, \alpha}-u_{\alpha}<\frac{1}{2} \delta$,

(ii) $\rho_{k, \alpha}>\left\{\frac{1}{2} \delta+\left(q^{2}-\delta^{2}\right)^{\frac{1}{2}}\right\}\left\{1-q^{-1}\left(q^{2}-\delta^{2}\right)^{\frac{1}{2}}\right\}^{-1}$,

(iii) $\sup _{\mu . \geq 0} R_{L R, \alpha}(\mu)<\varepsilon$,

and choose $\alpha^{\prime}, 0<\alpha^{\prime}<\alpha_{0}$, such that (2.4.21) holds. The set $\left\{x \mid\left[x_{i}^{2}<u_{\alpha},, x \geq 0\right\}\right.$ is a proper subset of $A_{\alpha}^{*}$, , since otherwise there would exist a supporting hyperplane of $A_{\alpha}$, at a distance smaller than $u_{\alpha}$, from the origin and the test would have a size larger than $\alpha^{\prime}$.

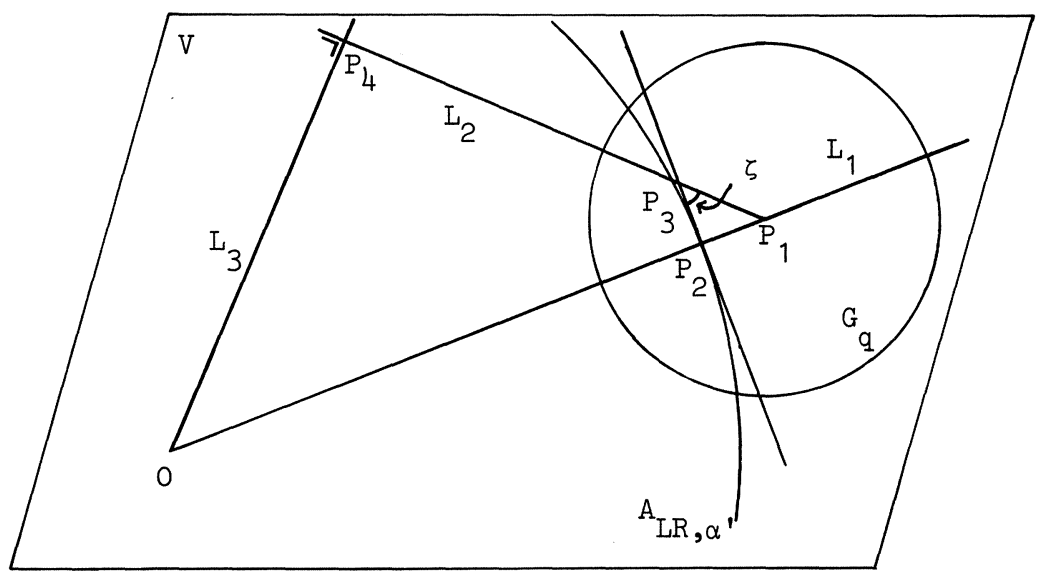

Fig. 2.4.1. Common boundary points of $A_{L R, \alpha}$, and $A_{\alpha^{\prime}}$, inside $G_{q}$. 
Hence by (i) $A_{L R, \alpha}^{*}, \subset U\left(A_{\alpha}^{*}, \frac{1}{2} \delta\right)$, the $\frac{1}{2} \delta$-neighborhood of $A_{\alpha}^{*}$. Therefore (2.4.21) implies the existence of a point $P_{1}=\left(x_{1}^{1}, \ldots, x_{k}^{\prime}\right)$ in the positive orthant on the boundary of $\mathrm{A}_{\alpha^{\prime}}$ at a distance $\rho_{\mathrm{k}, \alpha^{\prime}}+d>\rho_{\mathrm{k}, \alpha^{\prime}}+\delta$ from the origin. Let $\mathrm{L}_{1}$ be the line through the origin $O$ and $P_{1}$, and let $P_{2}$ be the point of intersection of $L_{1}$ and the boundary of $A_{L R, \alpha}^{*}$, . Define the region $G_{q}$ by

$$
G_{q}=\left\{x \mid \sum_{i=1}^{k}\left(x_{i}-x_{i}^{\prime}\right)^{2}<q^{2}\right\} \text {. }
$$

We shall show that the boundaries of $A_{\alpha}$, and $A_{L R, \alpha}$, have no common points in the set $G_{q}$. For suppose to the contrary that such a point would exist, say $P_{3}$. This would imply $P_{2} \in G_{q}$ and hence $d<q$. Denote the line through $P_{1}$ and $P_{3}$ by $L_{2}$ and let $\mathrm{V}$ be the plane through the points $\mathrm{P}_{1}, \mathrm{P}_{2}$ and $\mathrm{P}_{3}$; then the origin 0 also lies in V (see fig.2.4.1). Furthermore let $\zeta$ denote the positive acute angle between $\mathrm{L}_{2}$ and the line of support of $\mathrm{A}_{\mathrm{LR}, \alpha^{\prime}}$ in $\mathrm{V}$ through $\mathrm{P}_{2}$. Finally let $\mathrm{L}_{3}$ be the line through 0 orthogonal to $\mathrm{L}_{2}$ in $\mathrm{V}$ and let $\mathrm{P}_{4}$ be the point of intersection of $\mathrm{L}_{2}$ and $\mathrm{L}_{3}$. Then

$$
\overline{\mathrm{OP}}_{4}=\left(\rho_{\mathrm{k}, \alpha^{\prime}}+\alpha\right) \cos \zeta<\left(\rho_{\mathrm{k}, \alpha^{\prime}}+q\right) \cos \zeta .
$$

Since

$$
\sin \zeta=d /{\overline{P_{1} P_{3}}}_{3}>\delta / q,
$$

we have by (i) and (ii)

$$
\overline{\mathrm{OP}}_{4}<\left(\rho_{\mathrm{k}, \alpha^{\prime}}+\mathrm{q}\right)\left(1-\left(\frac{\delta}{\mathrm{q}}\right)^{2}\right)^{\frac{1}{2}}<\rho_{\mathrm{k}, \alpha^{\prime}}-\frac{1}{2} \delta<u_{\alpha^{\prime}} \cdot
$$

Since $P_{1}, P_{3}$ and $P_{4}$ are collinear and $P_{3}$ is situated between $P_{1}$ and $P_{4}, P_{4}$ lies outside $A_{\alpha}$, or on its boundary. This follows from the fact that $A_{\alpha}$, is convex and that $P_{1}$ and $P_{3}$ are boundary points. Therefore $\overline{O P}_{4} \geq u_{\alpha}$, contradicting (2.4.22). Hence the assertion that $A_{\alpha}$, and $A_{L R, \alpha}$, have no common boundary points in $G_{q}$ is proved.

It follows that $\left(G_{q}-A_{L R, \alpha^{\prime}}\right) \supset\left(G_{q}-A_{\alpha^{\prime}}\right)$ and that $\left(G_{q}-A_{L R, \alpha^{\prime}}\right)-\left(G_{q}-A_{\alpha^{\prime}}\right)$ contains a sector $C_{d}$ of a hypersphere with centre $P_{1}$ and radius $d$

$$
C_{d}=\left\{x \mid \sum_{i=1}^{k}\left(x_{i}-x_{i}^{\prime}\right)^{2}<d^{2}, x \leq x^{\prime}\right\} .
$$

Taking $\mu^{\prime}=x^{\prime}$, the definitions of $\varepsilon, q$ and $G_{q}$ imply 


$$
\begin{aligned}
\mathrm{R}_{\alpha^{\prime}}\left(\mu^{\prime}\right) & -\mathrm{R}_{L R, \alpha^{\prime}}\left(\mu^{\prime}\right)> \\
& >\mathrm{P}\left(\underline{\mathrm{x}} \in \mathrm{C}_{\mathrm{d}} \mid \mu^{\prime}\right)-\varepsilon \\
& >2^{-\mathrm{k}} \mathrm{P}\left(\sum_{i=1}^{\mathrm{k}} \underline{\mathrm{x}}_{i}^{2}\left\langle\delta^{2}\right| \mu=0\right)-\varepsilon=\varepsilon .
\end{aligned}
$$

Hence $R_{\alpha^{\prime}}\left(\mu^{\prime}\right)>\varepsilon$, contradicting (iii), which proves that (2.4.19) implies (2.4.20). To prove the converse, suppose to the contrary that $(2.4 .20)$ holds and that sequences $\left\{\alpha_{j}\right\}$ and $\left\{\mu^{(j)}\right\}$ exist such that $\lim _{j \rightarrow \infty} \alpha_{j}=0, \mu{ }^{(j)} . \geq 0$ for all $j=1,2, \ldots$ and

$$
R_{\alpha_{j}}\left(\mu^{(j)}\right)>\varepsilon \quad \text { for } j=1,2, \ldots
$$

where $\varepsilon$ is some positive number. Define $d>0$ by

$$
P\left(\sum_{i=1}^{k} \underline{x}_{i}^{2}>d \mid \mu=0\right)=\frac{1}{4} \varepsilon
$$

and let

$$
c_{j}=\left\{x \mid \sum_{i=1}^{k}\left(x_{i}-\mu_{i}^{(j)}\right)^{2} \leq d\right\} \quad, j=1,2, \ldots .
$$

Furthermore, let $D_{j}$ be the intersection of $C_{j}$ and the symmetric difference of $A_{\alpha_{j}}$ and $A_{L R, \alpha_{j}}$. Then

$$
(2.4 .26) \quad \lim _{j \rightarrow \infty} \lambda\left(D_{j}\right)=0 \text {, }
$$

where $\lambda$ denotes $k$-dimensional Lebesgue measure. Relation (2.4.26) is obvious from $\left(2.4 .20\right.$ ) if $C_{j}$ (and hence $D_{j}$ ) is a subset of the positive orthant. But the uniform convergence of the boundary of $A_{\alpha}$ to the boundary of $A_{L R, \alpha}$ in the positive orthant also ensures uniform convergence within the larger set $\left\{\mathrm{x}_{\mathrm{x}} \mid \mathrm{x}_{\mathrm{i}} \geq-\mathrm{d}, \mathrm{i}=1,2, \ldots, \mathrm{k}\right\}$. This may be verified by the same line of argument that we used in the first part of the proof to show that $G_{q}$ contained no common boundary points of $A_{\alpha}$, and $A_{L R, \alpha}$, Hence by (2.4.24) through (2.4.26)

$$
\left|R_{L R, \alpha}\left(\mu^{(j)}\right)-R_{\alpha_{j}}\left(\mu^{(j)}\right)\right|<\frac{1}{2} \varepsilon
$$

for all sufficiently large $j$. Since by lemma 2.4 .2 


$$
\lim _{j \rightarrow \infty} R_{L R, \alpha}\left(\mu^{(j)}\right)=0,
$$

(2.4.27) contradicts (2.4.23), showing that (2.4.20) indeed implies (2.4.19).

Q.E.D.

A well-known family of tests satisfying condition (2.4.20) of theorem 2.4.2 are FISHER's combination procedures which reject $\mathrm{H}$ if

$$
\text { (2.4.28) } \prod_{i=1}^{k}\left[1-\Phi\left(\underline{x}_{i}\right)\right] \leq c_{k, \alpha} \text {, }
$$

where $c_{k, \alpha}=\exp \left(-\frac{1}{2} x_{2 k, \alpha}^{2}\right)$. The shortcoming of FISHER's size- $\alpha$ test will be denoted by $R_{F, \alpha}$.

Lemma 2.4.3

FISHER's combination procedure $(2.4 .28)$ is admissible and its shortcoming $R_{F, \alpha}$ attains its absolute maximum exclusively on the edges of the parameter space.

Moreover,

$$
\lim _{\alpha \rightarrow 0} R_{F, \alpha}(\mu)=0 \quad \text { uniformly for } \mu . \geq 0
$$

Proof:

We write the acceptance region $A_{F, \alpha}$ of FISHER's size- $\alpha$ test as

$$
A_{F, \alpha}=\left\{x \mid x_{1}<a_{\alpha}\left(x_{2}, \ldots, x_{k}\right)\right\},
$$

where (omitting the index $\mathrm{k}$ in $\mathrm{c}_{\mathrm{k}, \alpha}$ )

$$
\text { (2.4.29) } \quad a_{\alpha}\left(x_{2}, \ldots, x_{k}\right)=-\Phi^{-1}\left(c_{\alpha} / \prod_{i=2}^{k} \Phi\left(-x_{i}\right)\right) \text {, }
$$

$\Phi^{-1}$ denoting the inverse function of $\Phi$. This is legitimate, since the test is obviously monotone. Furthermore it follows from the celebrated inequality

$$
(2.4 .30) \quad 1-\Phi(z)<\phi(z) / z \quad \text { for } z>0
$$

that $\log \Phi(-z)$ is concave in $z$ and hence

$$
\prod_{i=1}^{k} \Phi\left(-x_{i}^{\prime}\right) \geq c_{\alpha}, \prod_{i=1}^{k} \Phi\left(-x_{i}^{\prime \prime}\right) \geq c_{\alpha}
$$


imply

$$
\prod_{i=1}^{k} \Phi\left(-\gamma x_{i}^{\prime}-(1-\gamma) x_{i}^{\prime}\right) \geq c_{\alpha} \quad \text { for } 0<\gamma<1 \text {. }
$$

Thus $A_{F, \alpha}$ is monotone and convex, and the admissibility follows from lemma 1.4.6. of course $A_{F, \alpha}$ is also symmetric.

Now consider the function (cf.(2.3.9))

$$
g\left(x_{2}, \ldots, x_{k}\right)=x_{2}+a_{\alpha}\left(x_{2}, \ldots, x_{k}\right) \frac{\partial}{\partial x_{2}} a_{\alpha}\left(x_{2}, \ldots, x_{k}\right) .
$$

Let $x_{3}, x_{4}, \ldots, x_{k}$ be fixed, denote $c_{\alpha} / \prod_{3} \Phi\left(-x_{i}\right)$ by $d$ and suppress the variables $\mathrm{x}_{3}, \mathrm{x}_{4}, \ldots, \mathrm{x}_{\mathrm{k}}$ in the notation for $\mathrm{g}$. We shall show that $\mathrm{g}\left(\mathrm{x}_{2}\right)<0$ for $-\infty<\mathrm{x}_{2}<\mathrm{x}_{0}$, where $x_{0}=-\Phi^{-1}\left(d^{\frac{1}{2}}\right)$. We have

$$
g\left(x_{2}\right)=x_{2}+d \frac{\Phi^{-1}\left(d / \Phi\left(-x_{2}\right)\right)}{\phi\left(\Phi^{-1}\left(d / \Phi\left(-x_{2}\right)\right)\right)} \cdot \frac{\phi\left(x_{2}\right)}{\left\{\Phi\left(-x_{2}\right)\right\}^{2}} .
$$

As $A_{F, \alpha}$ is convex and monotone, the $\left(x_{3}, \ldots, x_{k}\right)$-section of $A_{F, \alpha}$ is also convex and monotone, implying that $a\left(x_{2}, \ldots, x_{k}\right)$ is a non-increasing and concave function of $\mathrm{x}_{2}$. The symmetry of $\mathrm{A}_{\mathrm{F}, \alpha}$ then implies that $-1<\partial \mathrm{a}_{\alpha}\left(\mathrm{x}_{2}, \ldots, \mathrm{x}_{\mathrm{k}}\right) / \partial \mathrm{x}_{2}<0$ for all $\mathrm{x}_{2}<\mathrm{x}_{0}$. With the aid of this relation and

$$
\Phi^{-1}\left(\mathrm{~d} / \Phi\left(-\mathrm{x}_{0}\right)\right)=\Phi^{-1}\left(\mathrm{~d}^{\frac{1}{2}}\right)=-\mathrm{x}_{0}
$$

it is easily verified that $g\left(x_{2}\right)$ is negative for $x_{2}<\min \left(0, x_{0}\right)$. Hence we restrict our attention to the case where $x_{0}>0$ and $0 \leq x_{2}<x_{0}$. Since $g\left(x_{0}\right)=0$, it is sufficient to prove that $g^{\prime}\left(x_{2}\right)>0$ for $0<x_{2}<x_{0}$. Differentiating $g$ with respect to $\mathrm{x}_{2}$ we obtain

$$
g^{\prime}\left(x_{2}\right)=1+\frac{d^{2}}{\left\{\phi\left(\Phi^{-1}\left(d / \Phi\left(-x_{2}\right)\right)\right)\right\}^{2}} \cdot \frac{\left\{\phi\left(x_{2}\right)\right\}^{2}}{\left\{\Phi\left(-x_{2}\right)\right\}^{4}}+
$$

$+d^{2} \frac{\left\{\Phi^{-1}\left(d / \Phi\left(-x_{2}\right)\right)\right\}^{2}}{\left\{\phi\left(\Phi^{-1}\left(d / \Phi\left(-x_{2}\right)\right)\right)\right\}^{2}} \cdot \frac{\left\{\phi\left(x_{2}\right)\right\}^{2}}{\left\{\Phi\left(-x_{2}\right)\right\}^{4}}-d \frac{\Phi^{-1}\left(d / \Phi\left(-x_{2}\right)\right)}{\phi\left(\Phi^{-1}\left(d / \Phi\left(-x_{2}\right)\right)\right)} \cdot \frac{x_{2} \phi\left(x_{2}\right)}{\left\{\Phi\left(-x_{2}\right)\right\}^{2}}+$

$+2 d \frac{\Phi^{-1}\left(d / \Phi\left(-x_{2}\right)\right)}{\phi\left(\Phi^{-1}\left(d / \Phi\left(-x_{2}\right)\right)\right)} \cdot \frac{\left\{\phi\left(x_{2}\right)\right\}^{2}}{\left\{\Phi\left(-x_{2}\right)\right\}^{3}}$.

Consider some fixed $x_{2}, 0<x_{2}<x_{0}$, and write $z=\Phi^{-1}\left(d / \Phi\left(-x_{2}\right)\right)$. 
Then

$$
z<\Phi^{-1}\left(\alpha / \Phi\left(-x_{0}\right)\right)=-x_{0}<0
$$

and $d=\Phi(z) \Phi\left(-x_{2}\right)$. In this notation

$$
\begin{aligned}
& \text { (2.4.31) } \quad g^{\prime}\left(x_{2}\right)=1-\frac{\mathrm{zx}_{2} \Phi(\mathrm{z})}{\phi(\mathrm{z})} \frac{\phi\left(\mathrm{x}_{2}\right)}{\Phi\left(-\mathrm{x}_{2}\right)}+ \\
& +\frac{\Phi(z)}{\phi(z)}\left[\frac{\phi\left(x_{2}\right)}{\Phi\left(-x_{2}\right)}\right]^{2}\left\{\frac{\Phi(z)}{\phi(z)}+z^{2} \frac{\Phi(z)}{\phi(z)}+2 z\right\} \text {. }
\end{aligned}
$$

We need the following inequalities

(2.4.32) $\Phi(-\mathrm{y})>\frac{\mathrm{y}}{1+\mathrm{y}^{2}} \phi(\mathrm{y}) \quad$ for $\mathrm{y}>0$,

(2.4.33) $\Phi(-y)>\frac{1}{2}\left(-y+\sqrt{y^{2}+4}\right) \phi(y) \quad$ for $y>0$.

The first inequality is due to Z.W. BIRNBAUM [6], the second inequality may be derived from the inequality

$$
\Phi(-y) / \phi(y)>R_{2}(y)=\left(2 y^{3}-4 y+2\left(y^{2}+12\right)\right) /\left(y^{4}+3\right)
$$

given by L.R. SHENTON [48]. Application of (2.4.32) to the expression between braces in (2.4.31) yields

$$
g^{\prime}\left(x_{2}\right)>1+\frac{z \Phi(z)}{\phi(z)}\left[\frac{\phi\left(x_{2}\right)}{\Phi\left(-x_{2}\right)}\right]^{2}\left\{1-\frac{x_{2} \Phi\left(-x_{2}\right)}{\phi\left(x_{2}\right)}\right\} .
$$

The expression between braces in the right-hand member is positive and hence, applying $(2.4 .30)$,

$$
\begin{aligned}
g^{\prime}\left(\mathrm{x}_{2}\right) & >1-\left[\frac{\phi\left(\mathrm{x}_{2}\right)}{\Phi\left(-\mathrm{x}_{2}\right)}\right]^{2}\left\{1-\frac{\mathrm{x}_{2} \Phi\left(-\mathrm{x}_{2}\right)}{\phi\left(\mathrm{x}_{2}\right)}\right\} \\
& =\left[\frac{\phi\left(\mathrm{x}_{2}\right)}{\Phi\left(-\mathrm{x}_{2}\right)}\right]^{2}\left\{-1+\mathrm{x}_{2} \frac{\Phi\left(-\mathrm{x}_{2}\right)}{\phi\left(\mathrm{x}_{2}\right)}+\left[\frac{\Phi\left(-\mathrm{x}_{2}\right)}{\phi\left(-\mathrm{x}_{2}\right)}\right]^{2}\right\} .
\end{aligned}
$$

The last expression of the right-hand member is positive by (2.4.33) and hence $g^{\prime}\left(x_{2}\right)>0$, implying $g\left(x_{2}\right)<0$ for $-\infty<x_{2}<x_{0}$. Then, by lemma 2.3.2, $R_{F, \alpha}$ assumes its absolute maximum only on the edges of the parameter space. 
Assume $\alpha<\frac{1}{2}$. Introducing polar coordinates $r, \omega_{1}, \ldots, \omega_{k-1}$ in $x$-space, the boundary of $\mathrm{A}_{\mathrm{F}, \alpha}$ may be written as $r=b_{\alpha}\left(\omega_{1}, \ldots, \omega_{k-1}\right)$. As $g\left(x_{2}, \ldots, x_{k}\right)<0$ for $-\infty<x_{2}<x_{0}\left(x_{3}, \ldots, x_{k}\right)$ and $A_{F, \alpha}$ is symmetric, $b_{\alpha}\left(\omega_{1}, \ldots, \omega_{k-1}\right)$ attains its maximum in the positive orthant for $\omega_{1}=\omega_{2}=\omega_{k-1}=\frac{1}{2} \pi$ and its minimum for $\omega_{1}=\arccos \mathrm{k}^{-\frac{1}{2}}, \omega_{2}=\arccos (\mathrm{k}-1)^{-\frac{1}{2}}, \ldots, \omega_{\mathrm{k}-1}=\arccos 2^{-\frac{1}{2}}=\frac{1}{4} \pi$. We denote these values by $\omega_{1}^{\prime}, \omega_{2}^{\prime}, \ldots, \omega_{k-1}^{\prime}$ respectively. Now consider the function

$$
\begin{aligned}
\Delta_{\alpha} & =b_{\alpha}\left(\frac{1}{2} \pi, \ldots, \frac{1}{2} \pi\right)-b_{\alpha}\left(\omega, \ldots, \omega_{k-1}^{\omega}\right) \\
& =\Phi^{-1}\left(c_{\alpha}{ }^{1 / k}\right) k^{\frac{1}{2}}-\Phi^{-1}\left(2^{k-1} c_{\alpha}\right) .
\end{aligned}
$$

From (2.4.30) we have

$$
\Phi(-\mathrm{v})<\mathrm{v}^{-1} \phi(\mathrm{v})<\mathrm{e}^{-\frac{1}{2} \mathrm{v}^{2}} \quad \text { for } \mathrm{v}>1,
$$

hence

$$
-v<\Phi^{-1}\left(e^{-\frac{1}{2} v^{2}}\right)
$$

and therefore

$$
\text { (2.4.35) } \quad \Phi^{-1}(w)>-(-2 \log w)^{\frac{1}{2}} \quad \text { for } 0<w<e^{-\frac{1}{2}}
$$

Let $\varepsilon$ be an arbitrary positive number. Then (2.4.32) yields

$$
\Phi(-v)>\frac{v}{1+v^{2}} \phi(v)>e^{-\frac{1}{2}(v+\varepsilon)^{2}}
$$

for all $\mathrm{v}>\mathrm{v}_{0}(\varepsilon)$. Hence

$$
\Phi^{-1}(\mathrm{w})<\varepsilon-(-2 \log \mathrm{w})^{\frac{1}{2}} \text { for } 0<\mathrm{w}<\mathrm{w}_{0}(\varepsilon) .
$$

Combining (2.4.34) through $(2.4 .36)$ we obtain

$$
\Delta_{\alpha}<\left\{-2 \log \left(2^{k-1} c_{\alpha}\right)\right\}^{\frac{1}{2}}-\left\{-\frac{2}{k} \log c_{\alpha}\right\}^{\frac{1}{2}}<\varepsilon k^{\frac{1}{2}}
$$

for all sufficiently small $c_{\alpha}$, i.e. for all $\alpha<\alpha_{0}(\varepsilon)$. Hence

$$
\lim _{\alpha \rightarrow 0} \Delta_{\alpha}=0
$$

Furthermore,

$$
u_{\alpha}<b_{\alpha}\left(w_{1}^{\prime}, \ldots, w_{k-1}^{\prime}\right)<\rho_{k, \alpha},
$$

where the last inequality is motivated by the fact that otherwise $A_{L R, \alpha}$ would be strictly contained in $A_{F, \alpha}$. Hence, because of $(2.4 .17), b_{\alpha}\left(\omega_{1}^{1}, \ldots \omega_{k-1}^{\prime}\right)-u_{\alpha}$ tends 
to zero for $\alpha \rightarrow 0$ and consequently (2.4.37) implies that condition (2.4.20) of theorem 2.4.2 is satisfied. Application of this theorem completes the proof.

Q.E.D.

The usefulness of asymptotic optimality for $\alpha \rightarrow 0$ of families of combination procedures, like the LR tests and the omnibus tests of FISHER, of course depends on the rate of convergence of the maximum shortcoming to zero. In this connection the following theorem is enlightening.

Theorem 2.4.3

For $\alpha \rightarrow 0$ and fixed $k \geq 2$

$$
\sup _{\mu \cdot \geq 0} R_{L R, \alpha}(\mu)=
$$

Proof:

$$
\begin{aligned}
& =(2 \pi)^{-\frac{1}{2}} u_{\alpha}^{-1}\left\{(k-1) \log u_{\alpha}-\frac{3}{2}(k-1) \log 2+\frac{1}{2} \log \pi-\log \Gamma\left(\frac{k}{2}\right)-\frac{1}{4}(k-1)\right\}+ \\
& +\mathscr{O}\left(u_{\alpha}^{-2} \log u_{\alpha}\right) .
\end{aligned}
$$

By lemma 2.4.2 the maximum shortcoming of the LR test is assumed at a point $\mu^{\alpha}=\left(\mu_{\alpha}, 0, \ldots, 0\right)$, where $\mu_{\alpha}$ depends on $\alpha$. First we consider

$$
1-\beta_{L R, \alpha}\left(\mu^{\alpha}\right)=\int_{0-}^{\rho_{\alpha}^{2}} \Phi\left(\left\{\rho_{\alpha}^{2}-t\right\}^{\frac{1}{2}}-\mu_{\alpha}\right) \mathrm{dF}(t),
$$

where $F$ is the distribution function of $\underline{t}^{\prime}{ }_{L R} \operatorname{def}^{\prime} \sum_{2}^{k} \underline{x}_{i}^{2} I_{(0, \infty)}\left(\underline{x}_{i}\right)$ under $H$ (we again omit the index $\mathrm{k}$ of $\left.\rho_{\mathrm{k}, \alpha}\right)$. We split the region of integration $\left[0, \rho_{\alpha}^{2}\right]$ into two parts: $\left[0,2\left(\rho_{\alpha}^{2}-u_{\alpha}^{2}\right)\right]$ and $\left(2\left(\rho_{\alpha}^{2}-u_{\alpha}^{2}\right), \rho_{\alpha}^{2}\right)$, and denote the corresponding integrals by $J_{1}$ and $J_{2}$ respectively. From $(2.4 .11)$ we derive

$$
\text { (2.4.39) } \rho_{\alpha}^{2}-u_{\alpha}^{2}=2(k-1) \log u_{\alpha}+2 a+\sigma\left(u_{\alpha}^{-1}\right) \quad \text { for } \alpha \rightarrow 0 \text {, }
$$

where $a=-\frac{3}{2}(k-1) \log 2+\frac{1}{2} \log \pi-\log \Gamma(k / 2)$. Hence, by an obvious modification of $(2.4 .16), P\left(\underline{t}_{L R}^{\prime}>2\left(\rho_{\alpha}^{2}-u_{\alpha}^{2}\right)\right)=\sigma\left(u_{\alpha}^{-2}\right)$ and therefore

$$
J_{2}=\sigma\left(u_{\alpha}^{-2}\right) \quad \text { for } \alpha \rightarrow 0 \text {. }
$$

If $0<t<2\left(\rho_{\alpha}^{2}-u_{\alpha}^{2}\right)$, then it is easily verified that for $\alpha \rightarrow 0$

$$
\left(\rho_{\alpha}^{2}-t\right)^{\frac{1}{2}}-u_{\alpha}=(k-1) u_{\alpha}^{-1} \log u_{\alpha}+a u_{\alpha}^{-1}-\frac{1}{2} u_{\alpha}^{-1} t+\sigma\left(u_{\alpha}^{-1} \log u_{\alpha}\right)
$$


and hence

$$
\begin{gathered}
\Phi\left(\left\{\rho_{\alpha}^{2}-t\right\}^{\frac{1}{2}}-\mu_{\alpha}\right)= \\
=\Phi\left(u_{\alpha}-\mu_{\alpha}\right)+\left(\left\{\rho_{\alpha}^{2}-t\right\}^{\frac{1}{2}}-u_{\alpha}\right) \phi\left(u_{\alpha}-\mu_{\alpha}\right)+\sigma\left(u_{\alpha}^{-2}\left(\mu_{\alpha}-u_{\alpha}\right) \phi\left(u_{\alpha}-\mu_{\alpha}\right) \log ^{2} u_{\alpha}\right) \\
=\Phi\left(u_{\alpha}-\mu_{\alpha}\right)+\left\{(k-1) u_{\alpha}^{-1} \log u_{\alpha}+a u_{\alpha}^{-1}-\frac{1}{2} u_{\alpha}^{-1} t\right\} \phi\left(u_{\alpha}-\mu_{\alpha}\right)+ \\
+\sigma\left(u_{\alpha}^{-2} \log u_{\alpha}\right)+\sigma\left(u_{\alpha}^{-2}\left(\mu_{\alpha}-u_{\alpha}\right) \phi\left(u_{\alpha}-\mu_{\alpha}\right) \log { }^{2} u_{\alpha}\right) .
\end{gathered}
$$

Since

$$
\begin{aligned}
& P\left(0 \leq t_{L R}^{\prime} \leq 2\left(\rho_{\alpha}^{2}-u_{\alpha}^{2}\right)\right)=1+\sigma\left(u_{\alpha}^{-2}\right) \text {, we find } \\
J_{1} & =\Phi\left(u_{\alpha}-u_{\alpha}\right)+\left\{(k-1) u_{\alpha}^{-1} \log u_{\alpha}+a u_{\alpha}^{-1}-\frac{1}{2} u_{\alpha}^{-1} \int_{0-}^{2\left(\rho_{\alpha}^{2}-u_{\alpha}^{2}\right)} \operatorname{tdF}(t)\right\} \phi\left(u_{\alpha}-\mu_{\alpha}\right)+ \\
& +\sigma\left(u_{\alpha}^{-2} \log u_{\alpha}\right)+\sigma\left(u_{\alpha}^{-2}\left(u_{\alpha}-u_{\alpha}\right) \phi\left(u_{\alpha}-u_{\alpha}\right) \log { }^{2} u_{\alpha}\right) .
\end{aligned}
$$

To determine the integral appearing in the right-hand member, we note that

$$
\int_{0-}^{2\left(\rho_{\alpha}^{2}-u_{\alpha}^{2}\right)} t d F(t)=E t_{L R}^{\prime}-\int_{2\left(\rho_{\alpha}^{2}-u_{\alpha}^{2}\right)}^{\infty} t d F(t) .
$$

By $(2.4 .12)$ and $(2 \cdot 4 \cdot 13) F^{\prime}(t)=C\left(e^{-\frac{1}{2} t} t^{\frac{1}{2}(k-3)}\right)$ for $t \rightarrow \infty$ and hence application of (2.4.39) yields that the last integral is $\left(g\left(u_{\alpha}^{-2} \log u_{\alpha}\right)\right.$.

Moreover

$$
E \underline{t}_{i R}^{\prime}=\sum_{i=2}^{k} E \underline{x}_{i}^{2} I(0, \infty)\left(\underline{x}_{i}\right)=\frac{1}{2}(k-1) .
$$

Therefore, for $\alpha \rightarrow 0$,

$$
\begin{aligned}
& \mathrm{R}_{\mathrm{LR}, \alpha}\left(\mu^{\alpha}\right)=J_{1}+J_{2}-\Phi\left(u_{\alpha}-\mu_{\alpha}\right)= \\
& =\left\{(k-1) u_{\alpha}^{-1} \log u_{\alpha}+a u_{\alpha}^{-1}-\frac{1}{4}(k-1) u_{\alpha}^{-1}\right\} \phi\left(u_{\alpha}-\mu_{\alpha}\right)+\theta\left(u_{\alpha}^{-2} \log u_{\alpha}\right)+ \\
& +\sigma\left(u_{\alpha}^{-2}\left(\mu_{\alpha}-u_{\alpha}\right) \phi\left(u_{\alpha}-\mu_{\alpha}\right) \log ^{2} u_{\alpha}\right) .
\end{aligned}
$$

This expression is maximized as a function of $\mu_{\alpha}$ if $\mu_{\alpha}$ satisfies $\mu_{\alpha}=u_{\alpha}+\sigma_{\left(u_{\alpha}^{-\frac{1}{2}}\right)}$. Substitution immediately yields the desired result.

Q.E.D.

It follows from this theorem that the leading terms of the maximum shortcoming 
of the LR test become small only for extremely small values of $\alpha$, because the rate of increase of $u_{\alpha}$ for $\alpha \rightarrow 0$ is very low (e.g. for $\alpha=10^{-5}$ we have $u_{\alpha}=4.26$ ). Hence the practical value of the asymptotic optimality for $\alpha \rightarrow 0$ of the LR tests remains somewhat dubious.

In this respect FISHER's omnibus tests do not behave any better. On the contrary, comparison of the acceptance regions of the LR tests and FISHER's tests suggests that on the edges of the parameter space FISHER's tests are slightly less powerful than the LR tests. Since both families of tests attain their maximum shortcoming on the edges of the parameter space, it may be expected that FISHER's tests have a larger maximum shortcoming that the LR tests. In fact, if the acceptance regions $\left\{A_{\alpha}\right\}$ of a family of admissible tests with shortcoming $\left\{R_{\alpha}\right\}$ do not approach the acceptance regions of the LR tests sufficiently fast for $\alpha \rightarrow 0$, the LR tests have the smaller maximum shortcoming for small values of $\alpha$. This refinement of theorem 2.4.2 is established in the following lemma. The set $A^{*}$ again denotes the intersection of a set $A$ and the positive orthant, and $U(A, \varepsilon)$ the $\varepsilon$-neighborhood of $A$.

\section{Lemma 2.4.4}

For any $k \geq 2$ there exists a positive number $\alpha_{0}(k)$ with the following property. If for some $\alpha<\alpha_{0}(k)$ the condition

$$
A_{\alpha}^{*} \subset U\left(A_{L R, \alpha}^{*}, \frac{1}{3} \pi^{\frac{1}{2}}(k+1)^{3 / 2} u_{\alpha}^{-1}\left(\log u_{\alpha}\right)^{\frac{1}{2}}\right)
$$

is not satisfied, then

$$
\sup _{\mu \cdot \geq 0} R_{\alpha}(\mu)>\sup _{\mu \cdot \geq 0} R_{L R, \alpha}(\mu)
$$

Proof:

We only give a sketch of the proof and omit some technical details. Let $P=\left(x_{1}^{\prime}, \ldots, x_{k}^{\prime}\right)$ be a point in $A_{\alpha}^{*}$ at a distance $u_{\alpha}+p_{\alpha}$ from the origin; we assume that $u_{\alpha}+p_{\alpha}>\rho_{k, \alpha}$ Let $\mathrm{C}_{\alpha}$ be the half-cone with vertex $\mathrm{P}$ tangent to the hypersphere $\mathrm{S}_{\alpha}$ with centre 0 (the origin) and radius $u_{\alpha}$, and let $\zeta$ be its semi-angle. Consider the alternative $\mu^{\prime}=x^{\prime}$, then

$$
R_{\alpha}\left(\mu^{\prime}\right)=\beta_{\alpha}^{+}\left(\mu^{\prime}\right)-\beta_{\alpha}\left(\mu^{\prime}\right),
$$

where $\beta_{\alpha}$ denotes the power function of the test with acceptance region $A_{\alpha}$ and 
$(2.4 .41)$

$$
\beta_{\alpha}^{+}\left(\mu^{\prime}\right)=\Phi\left(p_{\alpha}\right)>\frac{1}{2}+(2 \pi)^{-\frac{1}{2}} p_{\alpha}-(8 \pi)^{-\frac{1}{2}} p_{\alpha}^{3}
$$

To estimate $\beta_{\alpha}\left(\mu^{\prime}\right)$ we remark that the convex hull of $P$ and $S_{\alpha}$ is a subset of $\mathrm{A}_{\alpha}$ since $\mathrm{A}_{\alpha}$ is convex, $\mathrm{S}_{\alpha} \subset \mathrm{A}_{\alpha}$ and $\mathrm{P} \in \mathrm{A}_{\alpha}$. However, $\mathrm{C}_{\alpha}$ is not necessarily a subset of $A_{\alpha}$. Let $T_{\alpha}$ be the hypersphere with centre $P$ and radius $\left(u_{\alpha}+p_{\alpha}\right) \cos \zeta$, and let $G_{\alpha}$ be the set $C_{\alpha}-\left(S_{\alpha} \cup T_{\alpha}\right)$ (see fig.2.4.2). Then $A_{\alpha} \supset C_{\alpha}-G_{\alpha}$ and hence

$$
\begin{aligned}
\beta_{\alpha}\left(\mu^{\prime}\right) & =1-P\left(A_{\alpha} \mid \mu^{\prime}\right)<1-P\left(C_{\alpha}-G_{\alpha} \mid \mu^{\prime}\right)= \\
& =1-P\left(C_{\alpha} \mid \mu^{\prime}\right)+P\left(G_{\alpha} \mid \mu^{\prime}\right) .
\end{aligned}
$$

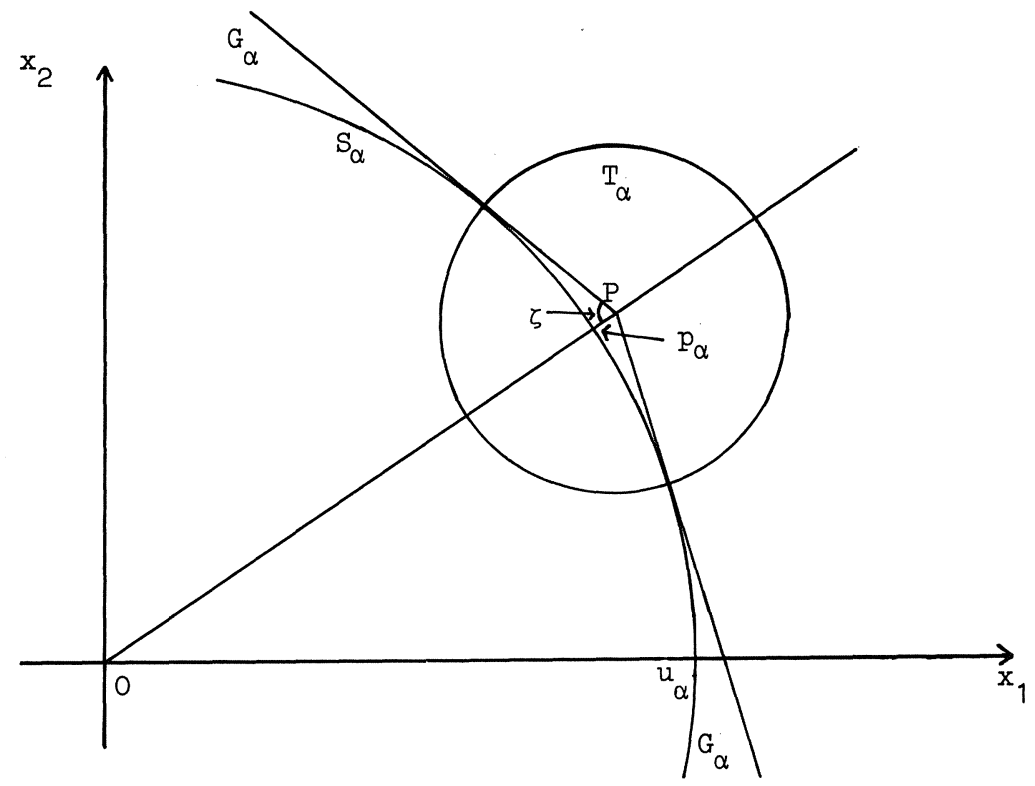

Fig. 2.4.2. The acceptance region $A_{\alpha}$ of the test in lemma 2.4 .4 for $k=2$. 
The solid angle subtended by $\mathrm{C}_{\alpha}$ (i.e. the surface area of the unit hypersphere with centre $P$ intercepted by $C_{\alpha}$ ) is equal to

$$
{ }^{B} \sin ^{2} \zeta\left(\frac{k-1}{2}, \frac{1}{2}\right) \cdot \pi^{\frac{1}{2}(k-1)} / \Gamma((k-1) / 2),
$$

where the first factor is the incomplete beta function

$$
\int_{0}^{\sin ^{2} \zeta} \mathrm{y}^{\frac{1}{2}(k-3)}(1-y)^{-\frac{1}{2}} d y .
$$

Since the surface area of a unit hypersphere in $\mathbb{R}^{\mathrm{k}}$ is equal to $2 \pi^{\frac{1}{2} \mathrm{k}} / \Gamma(\mathrm{k} / 2)$, we find that

$$
P\left(C_{\alpha} \mid \mu^{\prime}\right)=\frac{1}{2} B \sin ^{2} \zeta{ }^{\left(\frac{k-1}{2^{\prime}} \frac{1}{2}\right) / B\left(\frac{k-1}{2}, \frac{1}{2}\right) .}
$$

Evaluation of the incomplete beta function yields that for $0<\zeta<\frac{1}{2} \pi$

$$
\mathrm{P}\left(\mathrm{C}_{\alpha} \mid \mu^{\prime}\right)>\frac{1}{2}-\frac{1}{6}(\mathrm{k}+1) \cos \zeta,
$$

where

$$
\cos \zeta=\left\{1-u_{\alpha}^{2}\left(u_{\alpha}+p_{\alpha}\right)^{-2}\right\}^{\frac{1}{2}}=\left(2 u_{\alpha} p_{\alpha}+p_{\alpha}^{2}\right)^{\frac{1}{2}}\left(u_{\alpha}+p_{\alpha}\right)^{-1}<\left(2 p_{\alpha} u_{\alpha}^{-1}\right)^{\frac{1}{2}} .
$$

Moreover, it can be shown that

$$
P\left(G_{\alpha} \mid \mu^{\prime}\right)=\sigma\left(u_{\alpha}^{-2} \log u_{\alpha}\right) \text { for } u_{\alpha} \rightarrow \infty
$$

(this probability only depends on $u_{\alpha}$ and $p_{\alpha}$, not on $A_{\alpha}$ ). Let

$$
\mathrm{d}_{\alpha}=\rho_{\mathrm{k}, \alpha}+\frac{1}{3} \pi^{\frac{1}{2}}(\mathrm{k}+1)^{3 / 2} \mathrm{u}_{\alpha}^{-1}\left(\log \mathrm{u}_{\alpha}\right)^{\frac{1}{2}}
$$

and let $p_{\alpha}=d_{\alpha}-u_{\alpha}$. Then, by lemma 2.4.1,

$$
\mathrm{p}_{\alpha}=(\mathrm{k}-1) \mathrm{u}_{\alpha}^{-1} \log u_{\alpha}+\frac{1}{3} \pi^{\frac{1}{2}}(\mathrm{k}+1)^{3 / 2} u_{\alpha}^{-1}\left(\log u_{\alpha}\right)^{\frac{1}{2}}+\sigma\left(u_{\alpha}^{-1}\right)
$$

for $u_{\alpha} \rightarrow \infty$, and hence, from (2.4.41) and (2.4.42),

$$
\begin{aligned}
\mathrm{R}_{\alpha}\left(\mu^{\prime}\right) & >(2 \pi)^{-\frac{1}{2}} \mathrm{p}_{\alpha}-(8 \pi)^{-\frac{1}{2}} \mathrm{p}_{\alpha}^{3}-\frac{1}{6}(k+1)\left(2 \mathrm{p}_{\alpha} \mathrm{u}_{\alpha}^{-1}\right)^{\frac{1}{2}}-\mathrm{P}\left(\mathrm{G}_{\alpha} \mid \mu^{\prime}\right) \\
& >(2 \pi)^{-\frac{1}{2}}(\mathrm{k}-1) \mathrm{u}_{\alpha}^{-1} \log u_{\alpha}+\frac{1}{3} 2^{-\frac{1}{2}}(k+1)^{3 / 2} u_{\alpha}^{-1}\left(\log u_{\alpha}\right)^{\frac{1}{2}}+
\end{aligned}
$$




$$
\begin{aligned}
& -\frac{1}{6} 2^{\frac{1}{2}}(k+1)(k-1)^{\frac{1}{2}} u_{\alpha}^{-1}\left(\log u_{\alpha}\right)^{\frac{1}{2}}+\sigma\left(u_{\alpha}^{-1}\right) \\
= & (2 \pi)^{-\frac{1}{2}}(k-1) u_{\alpha}^{-1} \log u_{\alpha}+\frac{1}{6} 2^{\frac{1}{2}}(k+1)\left\{(k+1)^{\frac{1}{2}}-(k-1)^{\frac{1}{2}}\right\} u_{\alpha}^{-1} . \\
& \cdot\left(\log u_{\alpha}\right)^{\frac{1}{2}}+\mathcal{O}\left(u_{\alpha}^{-1}\right) .
\end{aligned}
$$

Hence, by theorem 2.4.3,

$$
\begin{aligned}
R_{\alpha}\left(\mu^{\prime}\right)-R_{L R, \alpha}\left(\mu^{\prime}\right)> & \frac{1}{6} 2^{\frac{1}{2}}(k+1)\left\{(k+1)^{\frac{1}{2}}-(k-1)^{\frac{1}{2}}\right\} u_{\alpha}^{-1}\left(\log u_{\alpha}\right)^{\frac{1}{2}}+ \\
& +O\left(u_{\alpha}^{-1}\right) .
\end{aligned}
$$

For sufficiently large $u_{\alpha}$, i.e. for sufficiently small $\alpha$, the right-hand member is positive. Let $\alpha_{0}(k)$ be a positive number such that it is positive for all $\alpha<\alpha_{0}(k)$.

Suppose condition (2.4.40) is not satisfied for some $\alpha<\alpha_{0}(k)$. Then there exists a point $P \in \mathrm{A}_{\alpha}^{*}$ at a distance $d_{\alpha}$ from the origin, where $d_{\alpha}$ is defined by (2.4.43). It follows that $R_{\alpha}\left(\mu^{\prime}\right)-R_{L R, \alpha}\left(\mu^{\prime}\right)>0$ and the lemma is proved. Q.E.D.

It turns out that FISHER's omnibus procedures do not satisfy condition (2.4.40) of lemma 2.4.4. Hence the maximum shortcoming of FISHER's tests is indeed larger than that of the LR procedures for sufficiently small values of $\alpha$. A proof of this assertion is omitted because of the rather complicated nature of the computations involved.

We now turn to other combination procedures for testing $\mathrm{H}$ against $\mathrm{K}$. First we consider the exponential procedures of theorem 2.3.1

By lemma 2.2.1 the parameter $r(\alpha)$ of these exponential procedures satisfies $r(\alpha)>u_{\alpha}$ and hence $r(\alpha) \rightarrow \infty$ for $\alpha \rightarrow 0$, implying $c(\alpha) \rightarrow \infty$ for $\alpha \rightarrow 0$. The boundary of the acceptance region $A_{\alpha}$ cuts the axis $x_{2}=\ldots=x_{k}=0$ at

$$
x_{1}=\frac{1}{r(\alpha)} \log (c(\alpha)-k+1)
$$

and cuts the line $x_{1}=x_{2}=\ldots=x_{k}$ at

$$
\mathrm{x}_{1}=\frac{1}{\mathrm{r}(\alpha)} \log (c(\alpha) / \mathrm{k}) .
$$


Taking the limit $\alpha \rightarrow 0$ we find that the difference of the expressions in the right-hand members converges to zero. Hence, recalling that $A_{\alpha}$ is symmetric, convex and monotone, we conclude that for $\alpha \rightarrow 0$ the exponential procedures approach TIPPETT's procedures which reject $\mathrm{H}$ for large values of the statistic

$$
\max \left(\underline{x}_{1}, \underline{x}_{2}, \ldots, \underline{x}_{k}\right)
$$

TIPPETT's test is admissible according to lemma 1.4.6; however, by theorem 2.2.1 it is not Bayes. As the exponential procedures of theorem 2.3.1 have a maximum shortcoming tending uniformly to one for $\alpha \rightarrow 0$ on every half-line through the origin in the positive orthant bounded away from the edges (corollary 2.4.1), the same holds for TIPPETT's procedures. The exponential procedures of theorem 2.3. 1 have uniformly vanishing shortcoming for $\alpha \rightarrow 0$ on the edges of the parameter space,because they are MS tests of $H$ against $\Omega_{\text {restr }}(c f .(2.3 .3))$ and hence their maximum shortcoming on $\Omega_{\text {restr }}$ is smaller than that of the LR tests which tends to zero for $\alpha \rightarrow 0$. It follows that TIPPETT's tests also have uniformly vanishing shortcoming on $\Omega_{\text {restr }}$.

Next we consider linear combination procedures which reject $\mathrm{H}$ if

$$
\text { (2.4.45) } \quad \sum_{i=1}^{k} v_{i} \underline{x}_{i} \geq u_{\alpha}\left(\sum_{i=1}^{k} v_{i}^{2}\right)^{\frac{1}{2}} \text {, }
$$

where $v=\left(v_{1}, \ldots, v_{k}\right) \geq 0$. As the test (2.4.45) is Bayes against any simple alternative on the half-line $\nu_{v}: \mu_{i}=\rho v_{i}(i=1,2, \ldots, k), \rho>0$, this test has for $\alpha \rightarrow 0$ limiting maximum shortcoming one that is reached on every half-line through the origin in the positive orthant with the exception of $L_{v}$ (cf.theorem 2.4.1). On $I_{v}$ the shortcoming is of course identically equal to zero for all $\alpha$.

In particular this is true for symmetric linear combination: reject $H$ if

$$
\text { (2.4.46) } \quad \sum_{i=1}^{k} \underline{x}_{i} \geq u_{\alpha} \sqrt{ } \text {. }
$$

For reasons of symmetry the maximum shortcoming of this test is smaller than that of any other linear combination procedure. Obviously the maximum shortcoming is reached on the $\mathrm{k}$ edges of the parameter space. Symmetric linear combination has been strongly advocated by SCHAAFSMA ([45], [46]), who calls $(2.4 .46)$ the most stringent somewhere most powerful (MSSMP) size- $\alpha$ test. 
Finally we consider the weighted FISHER procedures that reject $\mathrm{H}$ if

$$
\prod_{i=1}^{k}\left[1-\Phi\left(\underline{x}_{i}\right)\right]^{\nu} \leq c_{\alpha},
$$

where $\nu_{1}, \nu_{2}, \ldots, \nu_{k}$ are non-identical positive constants. These tests have been considered by I.J. GOOD [18], who derived a method for determining the critical value $c_{\alpha}$ in the case where all $v_{i}$ are different. Proceeding as in the proof of lemma 2.4.3 it can be shown that these tests are admissible. One would expect that the shortcoming of these tests is reached on one (or more) of the edges of the parameter space, but this is not easily proved since lema 2.3.2 does not apply. However that may be, we shall show that the maximum shortcoming of the test (2.4.47) tends to one for $\alpha \rightarrow 0$.

To simplify the notation, we assume without loss of generality that $\sum v_{i}=1$ and that $\nu_{1}>\nu_{2}>0$. The boundary of the acceptance region of the test $(2.4 .47)$ cuts the $x_{j}$-axis at

$$
x_{j, \alpha}=-\Phi^{-1}\left(\left[c_{\alpha} 2^{1-v_{j}}\right]^{1 / v_{j}}\right) \quad, j=1,2, \ldots, k .
$$

Hence, applying $(2.4 .35)$ and $(2.4 .36)$ with $\varepsilon=1$, we find

$$
\begin{aligned}
\mathrm{x}_{2, \alpha}-\mathrm{x}_{1, \alpha} & =\Phi^{-1}\left(\left[c_{\alpha}{ }^{1-\nu_{1}}\right]^{1 / \nu_{1}}\right)-\Phi^{-1}\left(\left[c_{\alpha} 2^{1-\nu_{2}}\right]^{1 / \nu_{2}}\right) \\
& >-\left[-2 v_{1}^{-1} \log \left(c_{\alpha} 2^{1-\nu_{1}}\right)\right]^{\frac{1}{2}}+\left[-2 v_{2}^{-1} \log \left(c_{\alpha} 2^{1-v_{2}}\right)^{\frac{1}{2}}-1\right.
\end{aligned}
$$

for sufficiently small $c_{\alpha}$. Since $c_{\alpha} \rightarrow 0$ for $\alpha \rightarrow 0$, the leading term of the last expression is $2^{\frac{1}{2}}\left(v_{2}^{-\frac{1}{2}}-v_{1}^{-\frac{1}{2}}\right)\left(-\log c_{\alpha}\right)^{\frac{1}{2}}$ and consequently $x_{2, \alpha}-x_{1, \alpha}+\infty$ for $\alpha \rightarrow 0$. As $x_{1, \alpha}>u_{\alpha}$, it follows that $x_{2, \alpha}-u_{\alpha} \rightarrow \infty$ for $\alpha \rightarrow 0$. Let $\mu^{(\alpha)}=\left(0, \mu_{\alpha}, 0, \ldots, 0\right)$, where $\mu_{\alpha}=\frac{1}{2}\left(u_{\alpha}+x_{2, \alpha}\right)$. Then it is easily verified that the shortcoming $R_{\alpha}\left(\mu^{(\alpha)}\right)$ of $(2.4 .47)$ at $\mu=\mu^{(\alpha)}$ tends to one for $\alpha \rightarrow 0$. 


\subsection{NUMERICAL COMPARISON OF SOME COMBINATION PROCEDURES}

In this section we study the shortcoming of some combination procedures for testing $\mathrm{H}$ against $\mathrm{K}$ in the normal case. The section is concluded with two remarks concerning more general combination methods. The procedures to be considered are

(i) the MS exponential procedures (2.3.4),

(ii) the LR tests $(2.4 .10)$,

(iii)FISHER's omnibus procedures (2.4.28),

(iv) symmetric linear procedures (2.4.46).

All computations were performed on the EL-X8 of the Mathematisch Centrum, with programs written in ALGOL 60. The figures were drawn by a plotter attached to the EL-X1.

First we make some remarks on the determination of the parameters $r(\alpha)$ and $c(\alpha)$ of the exponential procedures of theorem 2.3.1.

For $k=2$ we used the following method. For any given values $r$ and $c$ of the parameters the power function $\beta_{r, c}$ (or its derivative) in any given point was computed with the aid of a numerical integration procedure based on SIMPSON's rule. The repeated integrals were reduced to single integrals by direct approximation to the standard normal distribution function $\Phi$. Suppose the significance level $\alpha$ is given. Then for any given $r$ the corresponding critical value ${ }^{c=c}{ }_{r}$ of the test was determined as the zero of the function $\beta_{r, c}(0,0)-\alpha$ (considered as a function of $c$ ) by an iterative procedure. Finally $r(\alpha)$ was found as the unique zero of the function

$$
\left.\frac{\partial}{\partial \mu} R_{r, c}(\mu, 0)\right|_{\mu=r}=\phi\left(u_{\alpha}-r\right)-\left.\frac{\partial}{\partial \mu} \beta_{r, c}(\mu, 0)\right|_{\mu=r},
$$

where $R_{r, c}$ denotes the shortcoming of the exponential procedure with parameters $r$ and $c_{r}$. This process could easily be executed with high precision. Having found the parameters $r(\alpha)$ and $c(\alpha)$, it is very simple to compute the maximum shortcoming on the half-lines $\mu_{2}=0, \mu_{1}>0$ and $\mu_{1}=\mu_{2}>0$.

For $\mathrm{k}=3$ a more delicate approach was necessary, since the computation of the power functions and their derivatives involves repeated numerical integration, which is rather time-consuming if good precision is wanted. 
In this case a stepwise procedure was used, approximating $r(\alpha)$ and $c(\alpha)$ step by step and simultaneously modifying the tolerances of the numerical inetegration procedures at each step. Once $r(\alpha)$ and $c(\alpha)$ are determined, it is relatively easy to compute the maximum shortcoming on the three half-lines $\mu_{2}=\mu_{3}=0, \mu_{1}>0$; $\mu_{2}=\mu_{3}>0, \mu_{1}=0$ and $\mu_{1}=\mu_{2}=\mu_{3}>0$.

As was already mentioned in section 2.3, exponential combination turned out to be MS for $k=2, \alpha>\alpha_{0} \simeq 0.043$ and for $k=3, \alpha=0.1$, but not for $k=3, \alpha=0.05$. For $k=2, \alpha=0.1$ or $\alpha=0.05$ and for $k=3, \alpha=0.1$ the MS procedures are given by (2.3.14), $(2.3 .15)$ and $(2.3 .17)$ respectively.

The computation of the (maximum) shortcoming of the combination procedures (ii), (iii) and (iv) is much easier, because the test statistics do not depend on $\alpha$.

The boundaries of the acceptance regions of the four tests for $k=2$ and $\alpha=0.05$ and $\alpha=0.001$ are sketched in fig. 2.5.1 and fig 2.5.2 respectively; in the latter case exponential combination is omitted because it is not MS for $\alpha=0.001$.

In table 2.5.1 the maximum shortcoming of the four tests on the half-lines $\mu_{2}=0, \mu_{1}>0$ and $\mu_{1}=\mu_{2}>0$ is given for $k=2$ and a number of signifance levels $\alpha$. Table 2.5.2 shows the maximum shortcoming of these tests on the half-lines $\mu_{2}=\mu_{3}=0, \mu_{1}>0$ and $\mu_{1}=\mu_{2}=\mu_{3}>0$ for $k=3$ and some values of $\alpha$.

Exponential combination is only included for those values of $\alpha$ for which the procedure is MS. We recall that the procedures (i), (ii) and (iii) assume their maximum shortcoming on the edges of the parameter space (cf. section 2.4); whenever exponential combination is MS it also assumes its maximum shortcoming on this set (cf.section 2.3).

To obtain a better impression of the performance of the four tests than by their maximum shortcoming alone, the shortcoming of the tests has also been sketched with the aid of lines of equal shortcoming in the figures 2.5.3 through 2.5.6 for $k=2$ and $\alpha=0.05$ and in the figures 2.5.7 through 2.5 .9 for $k=2$ and $\alpha=0.001$ (exponential combination was again omitted in the latter case).

More numerical details about symetric linear combination and exponential combination are given in a report( $T W-40$ (1967), University of Groningen) by H.J. VAN LINDE, W. SCHAAFSMA and D. VELVIS.

Inspection of table 2.5.1 and the figures 2.5.3 through 2.5.9 permits us to draw the following tentative conclusions for the case $k=2$. 


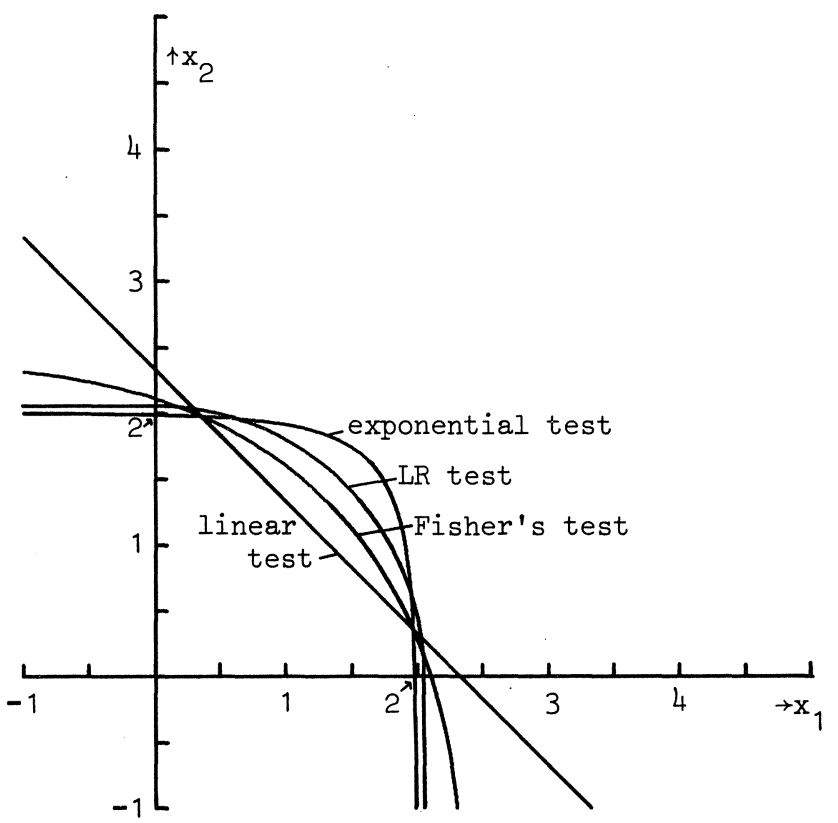

Fig. 2.5.1. Boundaries of the acceptance regions of 4 symmetric tests; size $\alpha=.05$.

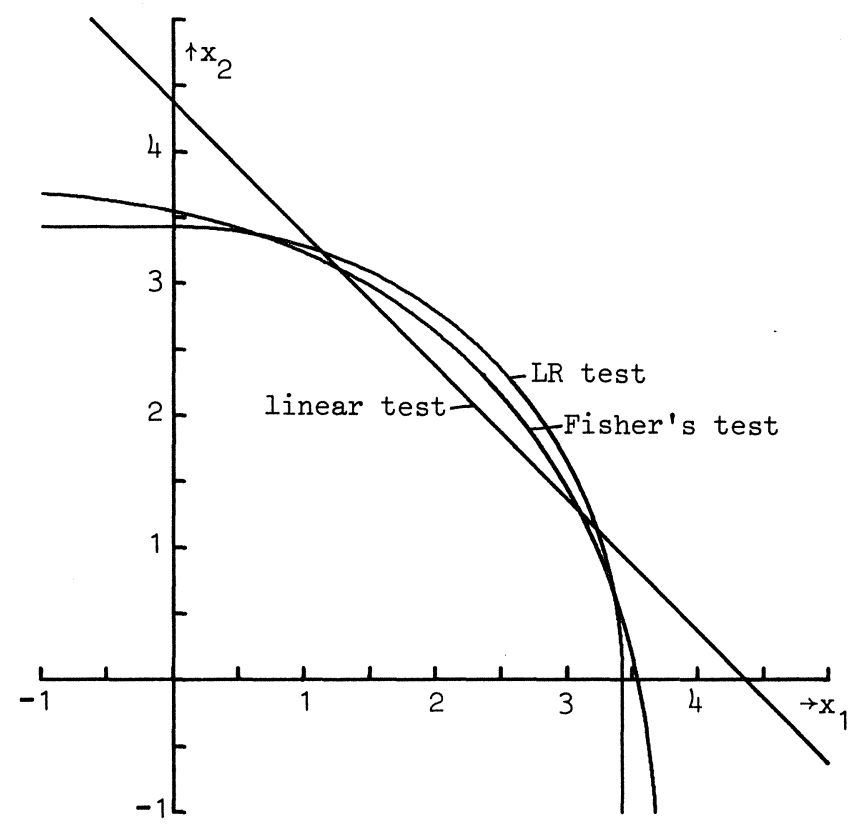

Fig. 2.5.2. Boundaries of the acceptance regions of 3 symmetric tests; size $\alpha=.001$. 
104

\begin{tabular}{|c|c|c|c|c|c|}
\hline size $\alpha$ & half-line & $\begin{array}{l}\text { exponential } \\
\text { combination }\end{array}$ & $\begin{array}{l}\text { IR } \\
\text { test }\end{array}$ & $\begin{array}{l}\text { FISHER's } \\
\text { procedure }\end{array}$ & $\begin{array}{l}\text { symmetric } \\
\text { linear comb. }\end{array}$ \\
\hline .1 & $\begin{array}{l}\mu_{2}=0, \mu_{1}>0 \\
\mu_{1}=\mu_{2}>0\end{array}$ & $\begin{array}{l}.108 \\
.068\end{array}$ & $\begin{array}{l}.108 \\
.059\end{array}$ & $\begin{array}{l}.121 \\
.022\end{array}$ & $\begin{array}{l}.213 \\
0\end{array}$ \\
\hline .05 & $\begin{array}{ll}\mu_{2}=0, & \mu_{1}>0 \\
\mu_{1}=\mu_{2} & >0\end{array}$ & $\begin{array}{l}.107 \\
.099\end{array}$ & $\begin{array}{l}.110 \\
.064\end{array}$ & $\begin{array}{l}.130 \\
.025\end{array}$ & $\begin{array}{l}.255 \\
0\end{array}$ \\
\hline .025 & $\begin{array}{l}\mu_{2}=0, \mu_{1}>0 \\
\mu_{1}=\mu_{2}>0\end{array}$ & & $\begin{array}{l}.110 \\
.068\end{array}$ & $\begin{array}{l}.136 \\
.028\end{array}$ & $\begin{array}{l}.292 \\
0\end{array}$ \\
\hline .01 & $\begin{array}{l}\mu_{2}=0, \quad \mu_{1}>0 \\
\mu_{1}=\mu_{2}>0\end{array}$ & & $\begin{array}{l}.109 \\
.072\end{array}$ & $\begin{array}{l}.141 \\
.030\end{array}$ & $\begin{array}{l}.334 \\
0\end{array}$ \\
\hline .005 & $\begin{array}{ll}\mu_{2}=0, & \mu_{1}>0 \\
\mu_{1}=\mu_{2} & >0\end{array}$ & & $\begin{array}{l}.108 \\
.073\end{array}$ & $\begin{array}{l}.143 \\
.032\end{array}$ & $\begin{array}{l}.363 \\
0\end{array}$ \\
\hline .001 & $\begin{array}{l}\mu_{2}=0, \mu_{1}>0 \\
\mu_{1}=\mu_{2}>0\end{array}$ & & $\begin{array}{l}.105 \\
.075\end{array}$ & $\begin{array}{l}.146 \\
.035\end{array}$ & $\begin{array}{l}.422 \\
0\end{array}$ \\
\hline .0001 & $\begin{array}{l}\mu_{2}=0, \mu_{1}>0 \\
\mu_{1}=\mu_{2}>0\end{array}$ & & $\begin{array}{l}.100 \\
.075\end{array}$ & $\begin{array}{l}.146 \\
.037\end{array}$ & $\begin{array}{l}.491 \\
0\end{array}$ \\
\hline
\end{tabular}

Table 2.5.1. Maximum shortcoming of 4 symmetric combination procedures for $\mathrm{k}=2$ on the half-lines $\mu_{2}=0, \mu_{1}>0$ and $\mu_{1}=\mu_{2}>0$.

For moderate values of $\alpha$ the maximum shortcoming of the LR procedure is barely larger than the shortcoming of the MS (exponential) test. For alternatives in the central part of the parameter space the LR test is slightly more powerful than the MS test for $\alpha=.1$ and .05. As the IR test approaches the MS test for $\alpha \rightarrow 0$ (theorem 2.4.2, cf. also lemma 2.4.4), we expect that the maximum shortcoming of the LR test will remain close to the minimax shortcoming for small values of $\alpha$. Hence the LR test may as well be applied as the more complicated MS test if a small maximum shortcoming is wanted. FISHER's test is somewhat more powerful than the LR test against alternatives in the central part of the parameter space and somewhat less powerful against alternatives along the edges. 


\begin{tabular}{|c|c|c|c|c|c|}
\hline size & half-line & $\begin{array}{l}\text { exponential } \\
\text { combination }\end{array}$ & $\begin{array}{l}\text { LR } \\
\text { test }\end{array}$ & $\begin{array}{l}\text { FISHER's } \\
\text { test }\end{array}$ & $\begin{array}{l}\text { symmetric } \\
\text { linear comb. }\end{array}$ \\
\hline \multirow[t]{2}{*}{.1} & $\mu_{2}=\mu_{3}=0, \mu_{1}>0$ & .167 & .170 & .193 & .327 \\
\hline & $\mu_{1}=\mu_{2}=\mu_{3}>0$ & .112 & .085 & .031 & 0 \\
\hline \multirow[t]{2}{*}{.05} & $\mu_{2}=\mu_{3}=0, \mu_{1}>0$ & & .175 & .209 & .389 \\
\hline & $\mu_{1}=\mu_{2}=\mu_{3}>0$ & & .094 & .035 & 0 \\
\hline \multirow[t]{2}{*}{.025} & $\mu_{2}=\mu_{3}=0, \mu_{1}>0$ & & .176 & .220 & .441 \\
\hline & $\mu_{1}=\mu_{2}=\mu_{3}>0$ & & .101 & .039 & 0 \\
\hline \multirow[t]{2}{*}{.01} & $\mu_{2}=\mu_{3}=0, \mu_{1}>0$ & & .176 & .229 & .500 \\
\hline & $\mu_{1}=\mu_{2}=\mu_{3}>0$ & & .108 & .043 & 0 \\
\hline \multirow[t]{2}{*}{.005} & $\mu_{2}=\mu_{3}=0, \mu_{1}>0$ & & .175 & .234 & .539 \\
\hline & $\mu_{1}=\mu_{2}=\mu_{3}>0$ & & .111 & .046 & 0 \\
\hline \multirow[t]{2}{*}{.001} & $\mu_{2}=\mu_{3}=0, \mu_{1}>0$ & & .172 & .240 & .615 \\
\hline & $\mu_{1}=\mu_{2}=\mu_{3}>0$ & & .116 & .050 & 0 \\
\hline
\end{tabular}

Table 2.5.2. Maximum shortcoming of 4 symmetric combination procedures for $k=3$ on the half-lines $\mu_{2}=\mu_{3}=0, \mu_{1}>0$ and $\mu_{1}=\mu_{2}=\mu_{3}>0$.

In section 2.4 it was shown that the maximum shortcoming of both FISHER's and the LR test tends to zero for $\alpha \rightarrow 0$; the numerical results indicate that this convergence is very slow (cf. lemma 2.4.3). Since the maximum shortcoming of both tests is not very large at the usual significance levels (about 11\% for the LR procedure and 12-14\% for FISHER's test), they appear to be satisfactory combination procedures for $k=2$ if no prior information about the alternatives is available. The shortcoming of the symmetric linear procedure shows a quite different pattern; this test is of course very powerful against alternatives in the central part of the parameter space but rather insensitive to alternatives close to the edges, especially for small values of $\alpha$ (cf. section 2.4). This suggests that symmetric linear combination should only be applied if there is some prior information indicating that $\mu_{1}$ and $\mu_{2}$ are not widely different. 


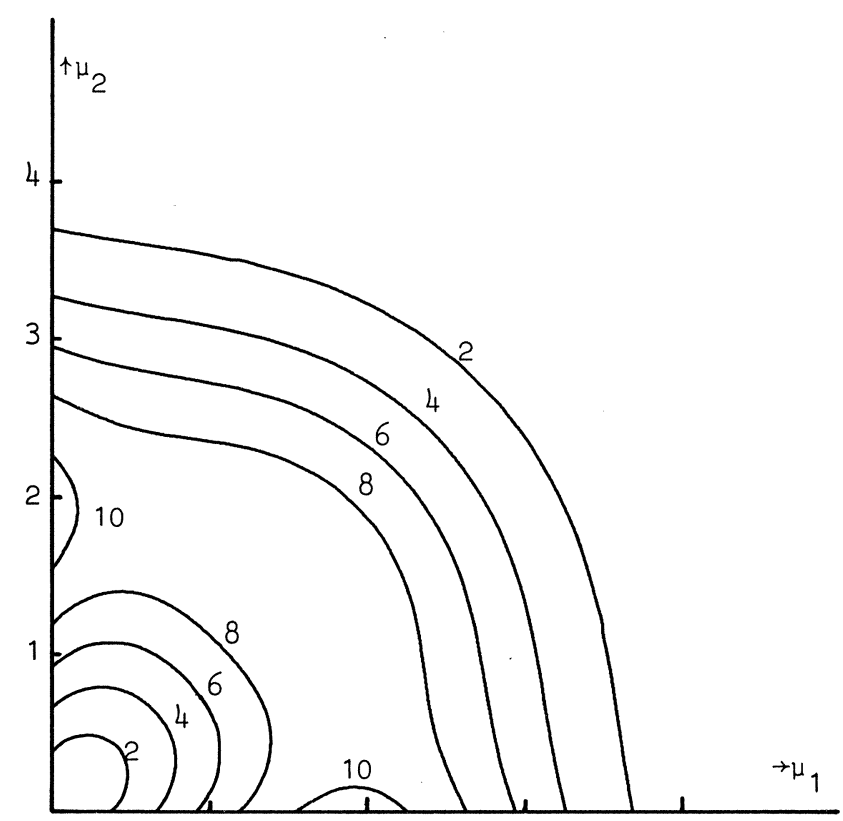

Fig. 2.5.3. Lines of equal shortcoming of the exponential procedure (in percents); size $\alpha=.05$ and $k=2$.

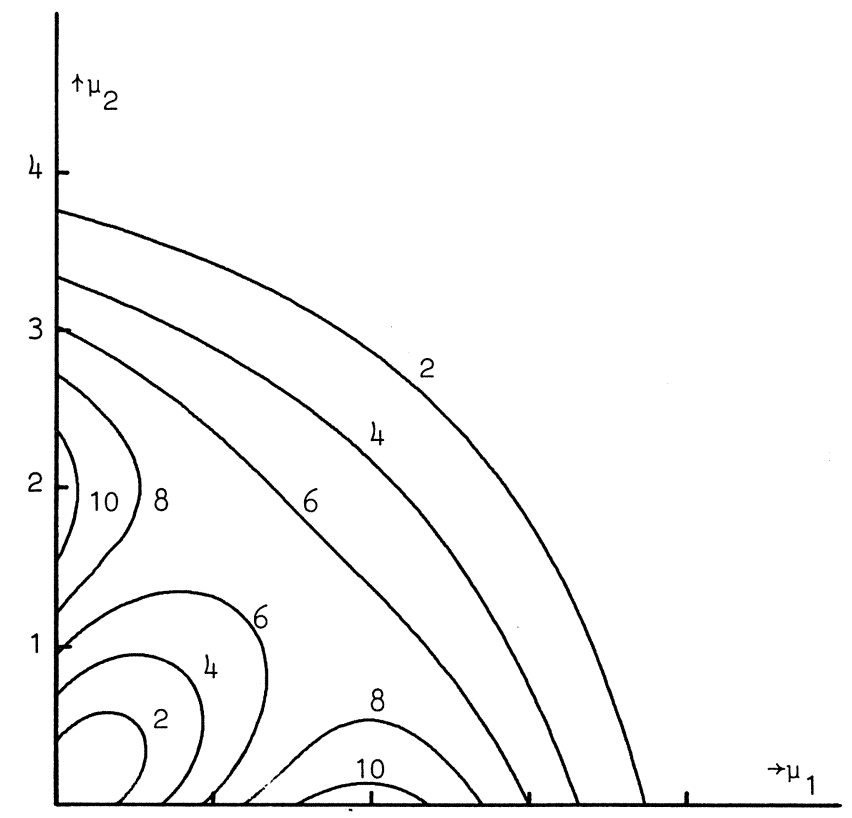

Fig. 2.5.4. Lines of equal shortcoming of the LR test (in percents); size $\alpha=.05$ and $k=2$. 
107

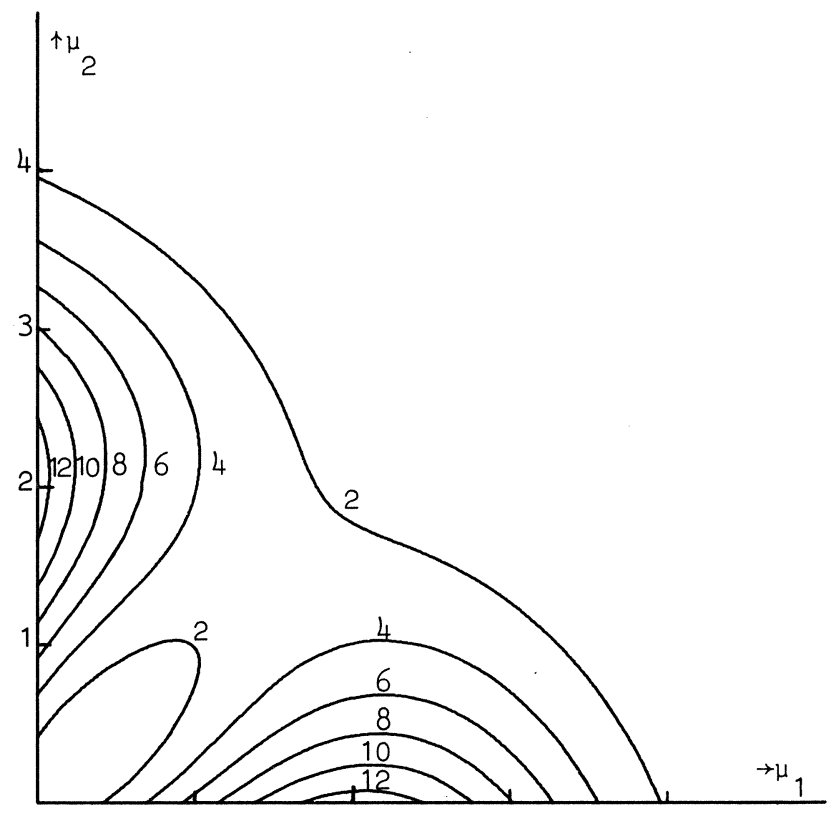

Fig. 2.5.5. Lines of equal shortcoming of FISHER's test (in percents); size $\alpha=.05$ and $\mathrm{k}=2$.

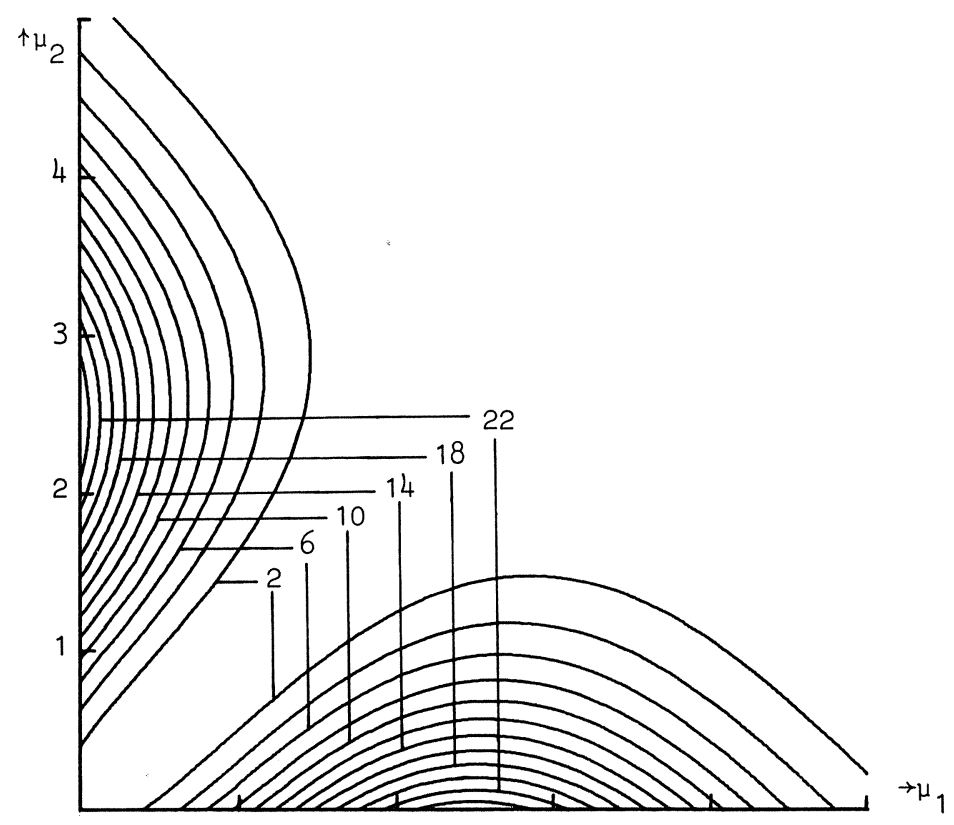

Fig. 2.5.6. Lines of equal shortcoming of the symmetric linear test (in percents); size $\alpha=.05$ and $\mathrm{k}=2$. 
108

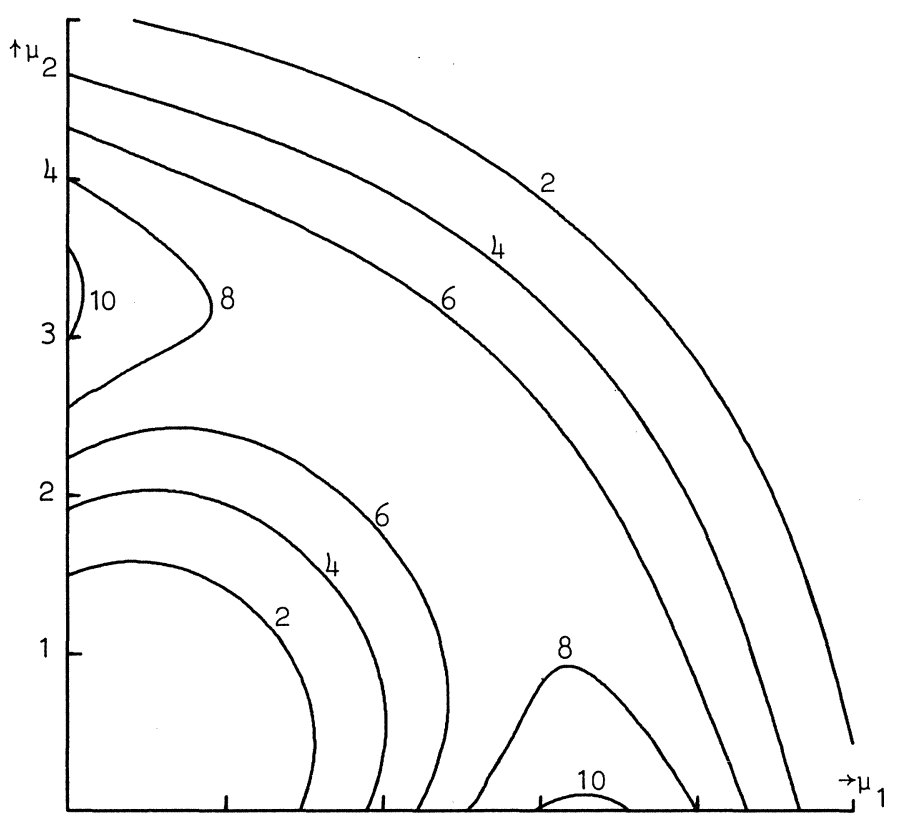

Fig. 2.5.7. Lines of equal shortcoming of the LR test (in percents); size $\alpha=.001$ and $k=2$.

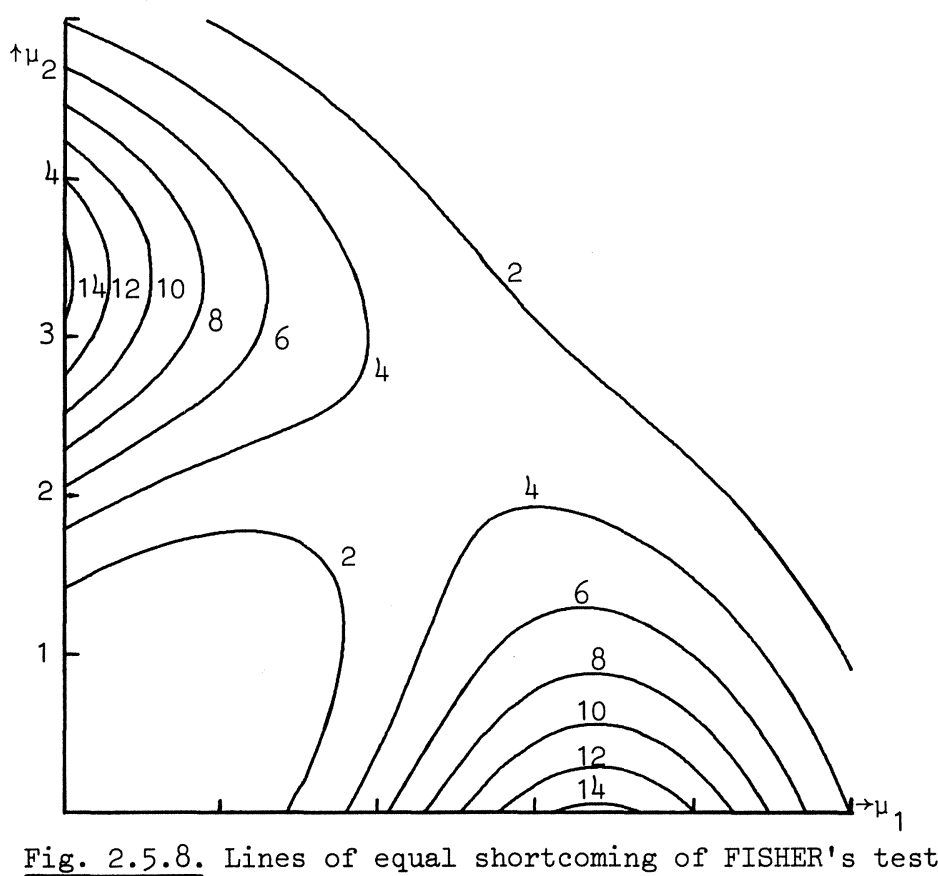

Fig. 2.5.8. Lines of equal shortcoming of FISHER's test (in percents); size $\alpha=.001$ and $k=2$. 


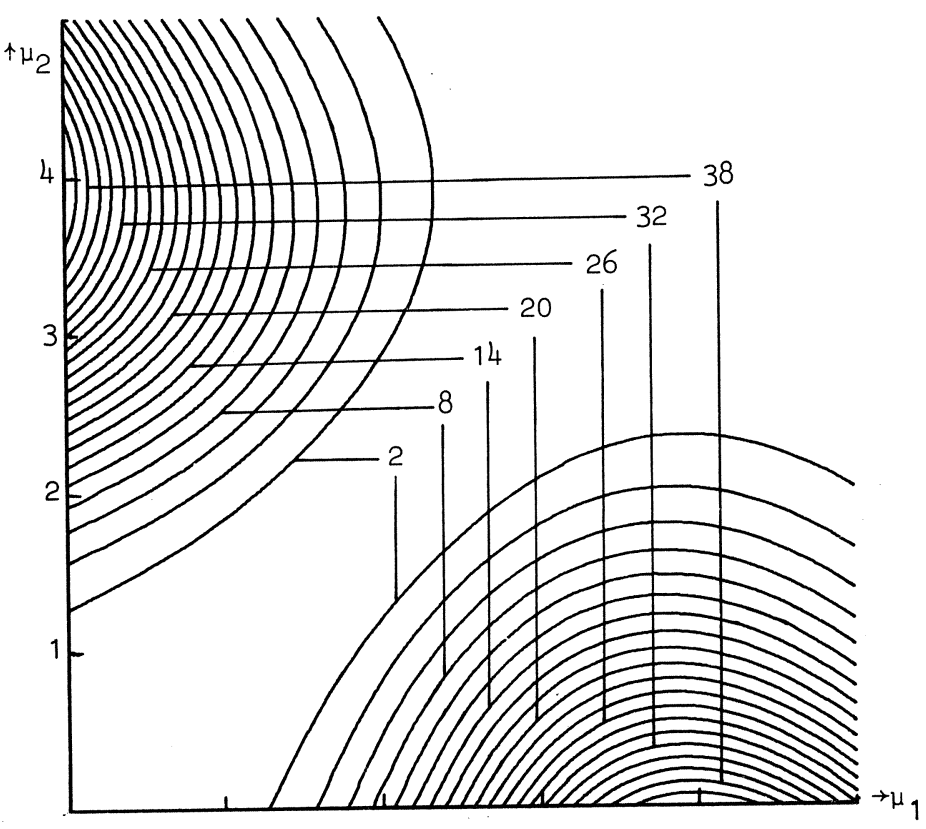

Fig. 2.5.9. Lines of equal shortcoming of the symmetric

linear test (in percents); size $\alpha=.001$ and $k=2$.

For $k=3$ numerical evidence is scarcer. It is seen from table 2.5.2 that in this case the maximum shortcoming of the tests considered is much larger than for $k=2$. Nevertheless, in the absence of prior information the LR test and FISHER's test seem to be relatively good combination procedures, FISHER's test being more powerful against alternatives in the central part of the parameter space and less powerful against alternatives near the edges.

For $k>3$ numerical data about FISHER's test and the LR procedure are not available. However, we expect that for increasing $\mathrm{k}$ the maximum shortcoming of these tests (and of any other test) will increase rapidly (cf.[46] th.5), and hence the advantage of a relatively small maximum shortcoming of the LR test and FISHER's test will diminish accordingly. One might say that, the larger $\mathrm{k}$, the more important it is to have some prior knowledge about most likely alternatives.

In section 1.6 linear combination procedures were considered for some wellknown non-normal combination problems. It was asserted (cf. page 41) that the test based on the sum of the standardized individual test statistics is optimal in some sense among all linear combination procedures in the large sample case. 
The individual test statistics considered in the examples of section 1.6 are asymptotically normal and hence, for increasing sample sizes, the shortcoming of the linear procedures is asymptotically equal to the shortcoming of the corresponding tests of $\mathrm{H}$ against $\mathrm{K}$ in the normal case by the theory of section 2.1. The test based on the sum of the standardized individual test statistics corresponds to the symmetric linear combination procedure (2.4.46) for testing $H$ against $K$. It follows that in the large sample case the test based on the sum of the standardized test statistics has a smaller maximum shortcoming than any other linear combination procedure. This property holds quite generally whenever the individual test statistics are asymptotically normal.

J. HEMELRIJK [21] suggested a different general approach to the combination problem if the densities of the individual test statistics $t_{i}$ belong to an exponential family of the form (1.6.2) and are based on samples of observations of size $n_{i}(i=1,2, \ldots, k)$. The parameter $\theta_{1}, \ldots, \theta_{k}$ being unknown, estimators $\hat{\theta}_{1}, \ldots, \hat{\theta}_{k}$ of the parameters may be obtained from the respective samples and the optimal test of $\mathrm{H}$ against $\theta=\left(\hat{\theta}_{1}, \ldots, \hat{\theta}_{k}\right)$ may be constructed $(c f .(1.6 .3))$. The resulting combination procedure (with $\hat{\theta}_{1}, \ldots, \hat{\theta}_{k}$ considered as random variables and due modification of the critical value) may be expected to possess good power properties in the large sample case if the method of estimation is efficient. Curiously enough it turns out that, for fixed $\alpha$, the maximum shortcoming of such procedures does not necessarily tend to zero for $n_{i} \rightarrow \infty(i=1,2, \ldots, k)$. In fact, these tests are often equivalent to LR tests.

\section{Example 2.5.1}

Let

$$
\underline{\mathrm{y}}_{1,1}, \ldots, \mathrm{y}_{1, \mathrm{n}_{1}} ; \ldots ; \mathrm{y}_{\mathrm{k}, 1}, \ldots, \mathrm{y}_{\mathrm{k}, \mathrm{n}_{\mathrm{k}}}
$$

be $\mathrm{k}$ samples of independent observations with normal $N\left(\mu_{i}, 1\right)$ distributions $(i=1,2, \ldots, k)$. The sample means $y_{1}, \ldots, y_{k}$. constitute a set of sufficient statistics and the MP test of $H: \mu=0$ against a simple alternative $\mu^{\prime} . \geq 0$ rejects $H$ for large values of the statistics $\sum_{i} n_{i}^{\frac{1}{2}} \mu_{i} y_{i}$. Substitution of the maximum likelihood estimators : $\hat{\underline{\mu}}_{i}=y_{i}$. if $y_{i}>0, \hat{\underline{\mu}}_{i}=0$ if $y_{i .} \leq 0(i=1,2, \ldots, k)$ yields the test that rejects $\mathrm{H}$ for large values. of the statistic

$$
\sum_{i=1}^{k} n_{i}^{\frac{1}{2}} y_{i}^{2} I_{(0, \infty)}\left(y_{i}\right) \text {, }
$$

i.e. the LR test of $\mathrm{H}$ against $\mathrm{K}: \mu . \geq 0$. Its maximum shortcoming is equal to that of the LR test (2.4.10). 
CHAPTER 3. COMBINATION OF ONE SAMPLE t-TESTS

3.1. OBSERVATIONS WITH EQUAL VARIANCES

Let

$$
\mathrm{y}_{1,1}, \ldots, \mathrm{y}_{1, \mathrm{n}_{1}} ; \ldots ; \mathrm{y}_{\mathrm{k}, 1}, \ldots, \mathrm{y}_{\mathrm{k}, \mathrm{n}_{\mathrm{k}}}
$$

be $k$ samples of independent observations with normal $N\left(\nu_{i}, \sigma_{i}^{2}\right)$ distributions $(i=1,2, \ldots, k)$. In this section we assume

$$
\sigma_{1}^{2}=\sigma_{2}^{2}=\ldots=\sigma_{k}^{2}=\sigma^{2} \text {. }
$$

One wishes to test the hypothesis

$$
\mathrm{H}^{*}: \nu=\left(\nu_{1}, \ldots, \nu_{\mathrm{k}}\right)=\nu^{0}, \sigma^{2}>0
$$

against the one-sided alternative

$$
K^{*}: \nu \cdot \geq \nu^{0}, \quad \sigma^{2}>0 \text {. }
$$

Here $\nu^{0}$ is a given vector and $\sigma^{2}$ an (unknown) nuisance parameter.

Then

$$
\underline{y}_{i .}=n_{i}^{-1} \sum_{j=1}^{n_{i}} y_{i, j}, \quad i=1,2, \ldots, k
$$

and

$$
\underline{s}^{2}=\sum_{i=1}^{k} \sum_{j=1}^{n_{i}}\left(\underline{y}_{i, j}-\underline{y}_{i}\right)^{2}
$$

constitute a set of sufficient statistics. Putting

$$
\begin{aligned}
\underline{x}_{i} & =\left(\underline{y}_{i}-v_{i}^{0}\right) \sqrt{ } n_{i}, i=1,2, \ldots, k, \\
\mu_{i} & =\left(v_{i}-v_{i}^{0}\right) \sqrt{ } n_{i}, i=1,2, \ldots, k, \\
N & =\sum_{i=1}^{k}\left(n_{i}-1\right),
\end{aligned}
$$

we obtain the following canonical form of the combination problem.

The random variables $\underline{x}_{1}, \underline{x}_{2}, \ldots, \underline{x}_{k}$ are independent and normally distributed with common variance $\sigma^{2}$ and expectations $\mathrm{Ex}_{i}=\mu_{i} \geq 0$ for $i=1,2, \ldots, k, \underline{s}^{2}$ is independent of the $\underline{x}_{i}$ and is distributed as $\sigma^{2} \underline{x}_{N}^{2}$, where $\underline{x}_{N}^{2}$ has a chi-square distribution with $\mathrm{N}$ degrees of freedom. The hypothesis to be tested is 


$$
\mathrm{H}: \mu=\left(\mu_{1}, \ldots, \mu_{k}\right)=0
$$

against

$$
\mathrm{K}: \mu \cdot \geq 0 \text {. }
$$

In section 2.1 (example 2.1.1) the large sample approach to this combination problem was considered. In the large sample case we may apply one of the combination methods of section 2.5 to the t-statistics

$$
\underline{t}_{i}=N^{\frac{1}{2}} \underline{x}_{i} / \underline{s}, \quad i=1,2, \ldots, k,
$$

because the $\underline{t}_{i}$ are asymptotically normal and independent for $N \rightarrow \infty$. In this section we study the small sample case.

Let

$$
\begin{aligned}
& \Omega_{\mathrm{H}}=\{(\mu, \sigma) \mid \mu=0, \sigma>0\}, \\
& \Omega_{\mathrm{K}}=\{(\mu, \sigma) \mid \mu=\mu . \geq 0, \sigma>0\},
\end{aligned}
$$

and consider the group $G$ of transformations $g$ of the sample space

$$
\left(\underline{x}_{1}, \ldots, \underline{x}_{k}, \underline{s}\right) \stackrel{g}{\rightarrow}\left(c \underline{x}_{1}, \ldots, c \underline{x}_{k}, c \underline{s}\right) \quad, c>0 .
$$

This group induces a group $\bar{G}$ of transformations $\bar{g}$ of the parameter space

$$
\left(\mu_{1}, \ldots, \mu_{k}, \sigma\right) \stackrel{\bar{g}}{\rightarrow}\left(c \mu_{1}, \ldots, c_{\mu_{k}}, c \sigma\right),
$$

which leaves $\Omega_{\mathrm{H}}$ and $\Omega_{\mathrm{K}}$ invariant. Obviously

$$
\underline{x}_{1} / \underline{s}, \cdots, \underline{x}_{k} / \underline{s}
$$

is a maximal invariant under $G$. The power function of any test based on this maximal invariant depends only on

$$
\mu_{1} / \sigma, \ldots, \mu_{k} / \sigma,
$$

the maximal invariant under $\bar{G}$ (cf.section 1.3).

It is well-known that the envelope power is a function of the maximal invariant under $\bar{G}$ only. Moreover, the envelope power remains unchanged if we test $H$ against the extended alternative

$$
K^{\prime}: \mu \neq 0, \sigma^{2}>0 \text {. }
$$

Since this testing problem is invariant under the group of orthogonal trans- 
formations applied to $\mu_{1} / \sigma, \ldots, \mu_{k} / \sigma$, the envelope power is a function of the maximal invariant

$$
\sum_{i=1}^{k} \mu_{i}^{2} / \sigma^{2}
$$

of this group only.

The envelope power can be determined by the same line of argument that E.L. LEHMANN and C.M. STEIN [32] used to derive the most powerful test of $\mathrm{H}$ against a simple alternative for $k=1$. It turns out that for $\alpha<\frac{1}{2}$ the most powerful size- $\alpha$ test of $H$ against the simple alternative $(\mu, \sigma)$ rejects $H$ if

$$
\begin{aligned}
& \sum_{i=1}^{k}\left[\underline{x}_{i}-\frac{1}{2} \mu_{i}\left\{1+\left(1+4(N+k) a^{2} \sigma^{2} M^{-1}\right)^{\frac{1}{2}}\right\}\right]^{2}+\underline{s}^{2} \leq \\
& \leq\left(1-b(N+k)^{-1}\right) M\left\{1+\left(1+4(N+k) a^{2} \sigma^{2} M^{-1}\right)^{\frac{1}{2}}\right\}^{2},
\end{aligned}
$$

where

$$
M=\sum_{i=1}^{k} \mu_{i}^{2}
$$

and $a$ and $b$ are positive numbers, depending on $N+k$ and $\alpha$, determined by the relations

$$
Q(a, b)=\sup _{a^{\prime}>0} Q^{\prime}\left(a^{\prime}, b\right)=\alpha, b<N+k,
$$

where

$$
Q(a, b)=P\left(\sum_{i=1}^{k}\left(\underline{u}_{i}-a\right)^{2} \leq(N+k-b) a^{2}\right)
$$

and $\underline{u}_{1}, \underline{u}_{2}, \ldots, \underline{u}_{k}$ are independent and normally $\mathbb{N}(0,1)$ distributed. Hence, for $\alpha<\frac{1}{2}$, the envelope power is equal to

$$
\begin{aligned}
& \beta_{\alpha}^{+}(\mu, \sigma)= \\
= & P\left(\underline{x}_{N+k}^{\prime 2} \leq\left(1-b(N+k)^{-1}\right) M \sigma^{-2}\left\{1+\left(1+4(N+k) a^{2} \sigma^{2} M^{-1}\right)^{\frac{1}{2}}\right\}^{2}\right),
\end{aligned}
$$

where $\underline{x}_{N+k}^{\prime 2}$ has a non-central chi-square distribution with $N+k$ degrees of freedom and non-centrality parameter

$$
\frac{1}{4} M \sigma^{-2}\left\{1-\left(1+4(N+k) a^{2} \sigma^{2} M^{-1}\right)^{\frac{1}{2}}\right\}^{2} .
$$

The complicated nature of the envelope power function suggests that without 
further restrictions combination methods with attractive theoretical properties will be hard to find.

In many testing problems the class of tests to be considered is restricted to unbiased, similar or invariant tests. In this connection it is enlightening to note that by lemma 1.3 .1 a MS test for testing $H$ against $K$ exists that is invariant under G.As our interest is focussed on tests with a small maximum shortcoming, this result suggests a restriction to tests which are invariant under G. At this point the question arises whether the shortcoming of an invariant test with respect to the envelope power (3.1.2) is still an adequate measure of its performance. It seems more natural to measure the shortcoming of invariant tests with respect to envelope invariant power, i.e. with respect to the supremum of the power taken over all invariant size- $\alpha$ tests.

Henceforth we shall restrict our attention to invariant tests,i.e. to tests based on the statistics $t_{1}, t_{2}, \ldots, t_{k}$, and measure their performance by the shortcoming with respect to the envelope invariant power function. Without loss of generality we assume that $\sigma^{2}=1$. The parameter space then reduces to the set $\{\mu \mid \mu \geq 0\}$. The joint density of $\underline{t}_{1}, \underline{t}_{2}, \ldots, \underline{t}_{k}$ is easily derived from the joint distribution of $\underline{x}_{1}, \underline{x}_{2}, \ldots, \underline{x}_{k}$ and $\underline{s}^{2}$; in the general case we find

$$
\begin{gathered}
p(t ; \mu)=p\left(t_{1}, \ldots, t_{k} ; \mu\right)= \\
=\left\{2^{\frac{1}{2}(N+k)}(\pi N)^{\frac{1}{2} k} \Gamma\left(\frac{1}{2} N\right)\right\}^{-1} \int_{0}^{\infty} v^{\frac{1}{2}(N+k-2)} \exp \left[-\frac{v}{2}-\frac{1}{2 N} \sum_{i=1}^{k}\left(t_{i} v^{\frac{1}{2}}-\mu_{i} N^{\frac{1}{2}}\right)^{2}\right] d v \text {, }
\end{gathered}
$$

where the integral can also be written as

$$
\begin{aligned}
& \exp \left(-\frac{1}{2} \sum_{i=1}^{k} \mu_{i}^{2}\right) \cdot\left[1+\frac{1}{N} \sum_{i=1}^{k} t_{i}^{2}\right]^{-\frac{1}{2}(N+k)} \cdot \\
& \cdot \int_{0}^{\infty} v^{\frac{1}{2}(N+k-2)} \exp \left[-\frac{v}{2}+\left(N+\sum_{i=1}^{k} t_{i}^{2}\right)^{-\frac{1}{2}} v^{\frac{1}{2}} \sum_{i=1}^{k} \mu_{i} t_{i}\right] d v \cdot
\end{aligned}
$$

Under $\mathrm{H}$ this density reduces to

$$
p(t ; 0)=\pi^{-\frac{1}{2} k} N^{\frac{1}{2} N} \frac{\Gamma\left(\frac{1}{2}(N+k)\right)}{\Gamma\left(\frac{1}{2} N\right)}\left(N+\sum_{i=1}^{k} t_{i}^{2}\right)^{-\frac{1}{2}(N+k)} .
$$

First we determine the most powerful invariant size- $\alpha$ test of $H$ against a simple alternative. 


\section{Lemma 3.1 .1}

The most powerful invariant size- $\alpha$ test of $\mathrm{H}$ against a simple alternative $\mu$ rejects $\mathrm{H}$ if

$$
\left(\sum_{i=1}^{k} \mu_{i} t_{i}\right)\left(N+\sum_{i=1}^{k} t_{i}^{2}\right)^{-\frac{1}{2}} \geq d_{\alpha},
$$

where

$$
a_{\alpha}=t_{N+k-1 ; \alpha}\left(\sum_{i=1}^{k} \mu_{i}^{2}\right)^{\frac{1}{2}}\left(N+k-1+t_{N+k-1 ; \alpha}^{2}\right)^{-\frac{1}{2}}
$$

and $t_{v ; \alpha}$ denotes the upper $\alpha$-point of the $t$-distribution with $v$ degrees of freedom.

Proof:

From the NEYMAN-PEARSON lemma and (3.1.3) through (3.1.5) we find that the most powerful (MP) invariant size- $\alpha$ test of $H$ against $\mu$ rejects $H$ for large values of the statistic

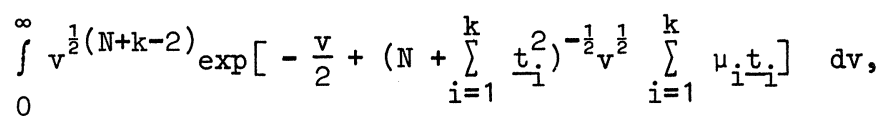

that is, for large values of $\left(N+\sum_{i} t_{i}^{2}\right)^{-\frac{1}{2}}\left[\mu_{i} t_{i}\right.$. To determine the critical value $d_{\alpha}$, we note that under $H$ the distribution of the vector $t$ is rotation invariant (cf. $(3 \cdot 1 \cdot 5))$. Hence

$$
\begin{aligned}
P\left(\sum_{i=1}^{k} \mu_{i} \underline{t}_{i}\left(N+\sum_{i=1}^{k} \frac{t_{i}^{2}}{)^{-\frac{1}{2}}} \geq d_{\alpha} \mid . H\right)\right. & = \\
& =P\left(\underline{t}_{-1}\left(N+\sum_{i=1}^{k} t_{i}^{2}\right)^{-\frac{1}{2}} \geq d_{\alpha}^{*} \mid H\right),
\end{aligned}
$$

where $d_{\alpha}^{*}=d_{\alpha}\left(\sum_{i} \mu_{i}^{2}\right)^{-\frac{1}{2}}$. Since the event

$$
\underline{t}_{1}\left(N+\sum_{i=1}^{k} \frac{t_{i}^{2}}{)^{-\frac{1}{2}}} \geq d_{\alpha}^{*}\right.
$$

is equivalent to

$$
\underline{t}_{1}\left(N+\sum_{i=2}^{k} t_{i}^{2}\right)^{-\frac{1}{2}} \geq d_{\alpha}^{*}\left(1-d_{\alpha}^{* 2}\right)^{-\frac{1}{2}}
$$

and hence to 


$$
\underline{x}_{1}\left(\underline{s}^{2}+\sum_{i=2}^{k} \underline{x}_{i}^{2}\right)^{-\frac{1}{2}} \geq d_{\alpha}^{*}\left(1-d_{\alpha}^{* 2}\right)^{-\frac{1}{2}},
$$

$d_{\alpha}^{*}$ obviously must be solved from the equation

$$
d_{\alpha}^{*}\left(1-d_{\alpha}^{* 2}\right)^{-\frac{1}{2}}=(N+k-1)^{-\frac{1}{2}} t_{N+k-1 ; \alpha}
$$

This immediately yields (3.1.7).

The class of MP invariant size- $\alpha$ tests has also been obtained (in a different notation) by W. SCHAAFSMA [45] (cf. also [44]), who proved that the tests (3.1.6) are in fact the $\mathbb{M P}$ similar size- $\alpha$ tests of $\mathrm{H}$ against simple alternatives.

Performing an orthogonal transformation of the sample space it is easily verified that the envelope invariant power function $\beta_{\alpha}^{+}$,inv only depends on $\sum_{i} \mu_{i}^{2}$ and is equal to

$$
\beta_{\alpha, \text { inv }}^{+}(\mu)=P\left(\underline{t}_{N+k-1}^{\prime} \geq t_{N+k-1 ; \alpha}\right),
$$

where $t_{N+k-1}^{\prime}$ has a non-central $t$-distribution with $N+k-1$ degrees of freedom and non-centrality parameter $\left(\sum_{i} \mu_{i}^{2}\right)^{\frac{1}{2}}$.

The critical region of the test (3.1.6) is, for $\alpha<\frac{1}{2}$, one solid sheet of a hyperbole of revolution in $t$-space. The tangent half-cone of this sheet has vertex $O$ (the origin), an axis orthogonal to the hyperplane $\sum_{i} \mu_{i} t_{i}=0$ and semi-angle

$$
\operatorname{arc} \operatorname{tn}\left\{(N+k-1)^{\frac{1}{2}} / t_{N+k-1} ; \alpha\right\}
$$

SCHAAFSMA [45] has shown that the symmetric test which rejects $H$ if

$$
\sum_{i=1}^{k} \underline{t}_{i}\left(N+\sum_{j=1}^{k} t_{j}^{2}\right)^{-\frac{1}{2}} \geq d_{\alpha}
$$

is MS among the class of tests (3.1.6); he calls it the MSSMP similar size- $\alpha$ test. He also demonstrated that a unique MSSMP similar size- $\alpha$ test exists if and only if the condition

$$
(N+k-1) \geq(k-1) t_{N+k-1 ; \alpha}^{2}
$$


is satisfied; if not, the solid tangent half-cone of the critical region defined by (3.1.9) is a proper subset of the positive orthant and the maximum shortcoming of every MP invariant size- $\alpha$ test is equal to one. In applications $\alpha$ will not often be so small that the inequality (3.1.10) is not satisfied. Nevertheless, compared with the symmetric linear size- $\alpha$ test procedure $(2.4 .46)$ in the normal case with unit variances, the test (3.1.9) undoubtedly has a stronger tendency to concentrate its power on alternatives near the central half-line $\mu_{1}=\mu_{2}=\ldots=\mu_{k}>0$ of the positive orthant and hence it does not appear to be very satisfactory from an overall point of view (SCHAAFSMA does not share this opinion, cf. [45] ch.4).

In the case of known variances treated in chapter 2 the admissible tests for the one-sided combination problem were characterized as the tests with a.e. convex and monotone acceptance regions (cf. lemma 1.4.6). This result does not immediately extend to the present problem, as is demonstrated by the Bayes tests (3.1.6) whose acceptance regions are neither convex nor monotone. However, by applying the transformation

$$
\underline{z}_{i}=\underline{t}_{i}\left(N+\sum_{j=1}^{k} t_{j}^{2}\right)^{-\frac{1}{2}}, \quad i=1,2, \ldots, k,
$$

we obtain a theorem similar to BIRNBAUM's result. We note that under this transformation $\left(N+\sum_{i} t_{i}^{2}\right)$ transforms into $N\left(1-\sum_{i} z_{i}^{2}\right)^{-1}$ and that the Jacobian of the transformation is equal to

$$
\mathrm{N}^{\frac{1}{2} \mathrm{k}}\left(1-\sum_{i=1}^{\mathrm{k}} \mathrm{z}_{i}^{2}\right)^{\frac{1}{2} \mathrm{k}-1}
$$

Hence we find from (3.1.3) and (3.1.4) that in the general case the density of $\underline{z}_{1}, \underline{z}_{2}, \cdots, \underline{z}_{k}$ is given by

$$
f(z ; \mu)=f\left(z_{1}, \ldots, z_{k} ; \mu\right)=
$$

$$
=\exp \left(-\frac{1}{2} \sum_{i=1}^{k} \mu_{i}^{2}\right) h\left(z_{1}, \ldots, z_{k}\right) \int_{0}^{\infty} v^{\frac{1}{2}(N+k-2)} \exp \left[-\frac{v}{2}+v^{\frac{1}{2}} \sum_{i=1}^{k} \mu_{i} z_{i}\right] d v
$$

for $\sum_{i=1}^{k} z_{i}^{2}<1$ and 0 elsewhere, where

$$
h\left(z_{1}, \ldots, z_{k}\right)=\left\{2^{\frac{1}{2}(N+k)} \pi^{\frac{1}{2} k} \Gamma\left(\frac{1}{2} N\right)\right\}^{-1} \cdot\left(1-\sum_{i=1}^{k} z_{i}^{2}\right)^{\frac{1}{2} N+k-1} .
$$


We note that the MP invariant size- $\alpha$ tests (3.1.6) transform into linear procedures in $z$-space which reject $H$ if

$$
\sum_{i=1}^{k} \mu_{i} z_{i} \geq d_{\alpha} .
$$

\section{Theorem 3.1.1}

The class $W_{\alpha}$ of size- $\alpha$ tests of $H$ with a.e. convex and monotone acceptance regions in $\mathrm{z}$-space is minimal complete for testing $\mathrm{H}$ against $\mathrm{K}$ among invariant size- $\alpha$ tests.

\section{Proof:}

To prove the theorem we essentially use BIRNBAUM's approach (cf.[5]). The proof is given in several steps; technical details are omitted. (i)since $f(z ; \mu) / f(z ; 0)$ is convex in $z$ and $f(z ; \mu)$ has strict MLR in $z$, theorems 1.4 .1 and 1.4.3 imply that $W_{\alpha}$ is essentially complete.(ii) The class $W_{\alpha}$ is complete. To prove this property it is convenient to return to the class of all, not necessarily invariant, size- $\alpha$ tests and to the original parameter space (with $\left.\sigma^{2}>0\right)$. The joint density of $\underline{x}_{1}, \underline{x}_{2}, \ldots, \underline{x}_{k}, \underline{s}^{2}$ is an exponential family with parameters $\mu_{1} / \sigma^{2}, \mu_{2} / \sigma^{2}, \ldots, \mu_{k} / \sigma^{2}$ and $1 / \sigma^{2}$. Since such a family is boundedly complete, equality of the power functions of two size- $\alpha$ tests implies equality of their critical functions a.e. As the invariant tests are a subclass of all size- $\alpha$ tests, essential completeness of $\mathrm{W}_{\alpha}$ implies completeness of $\mathrm{W}_{\alpha}$. (iii) The class $\mathrm{W}_{\alpha}$ minimal complete. Since thesample space is the solid unit hypersphere and hence bounded, the same argument that BIRNBAUM used to prove theorem 3 of [5] can be adapted to prove minimal completeness of $w_{\alpha}$. BIRNBAUM's assumption that the distributions of the statistics constitute an exponential family is not satisfied by the joint distribution of $\underline{z}_{1}, \underline{z}_{2}, \ldots, \underline{z}_{k}$, but their distribution possesses all the properties that are required in BIRNBAUM's proof.

Q.E.D.

In chapter 2 it was shown (theorem 2.2.2) that in the case of exponential family distributions under some mild conditions the shortcoming of every admissible test has a unique maximum on each half-line through the origin in the parameter space. Since the proof of theorem 2.2.2 hinges on the independence of the random variables, it cannot be adapted to the present problem and it is 
still unknown whether the shortcoming of admissible invariant procedures has unique maxima on half-lines through the origin in the parameter space. This implies that the construction of MS tests by the method of section 2.3 cannot be justified here.

However, a lemma similar to lemma 2.3.2 does hold for the present combination problem.

Lemma 3.1 .2

Let $A$ be the acceptance region of an invariant test $\delta$ in t-space satisfying the following conditions:

(i) the set $A$ is symmetric in $t_{1}, t_{2}, \ldots, t_{k}$;

(ii) the set $A$ is monotone; this implies the existence of a function a such that $A$ can be written as

$$
A=\left\{t \mid t_{1}<a\left(t_{2}, \ldots, t_{k}\right)\right\} ;
$$

(iii) the function a defined in (ii) has continuous first order partial derivatives on the interval where a is finite.

Let the functions $g$ and $t_{0}$ be defined by

$$
\begin{aligned}
& g\left(t_{2}, \ldots, t_{k}\right)=t_{2}+a\left(t_{2}, \ldots, t_{k}\right) \cdot \frac{\partial}{\partial t_{2}} a\left(t_{2}, \ldots, t_{k}\right), \\
& a\left(t_{0}\left(t_{3}, \ldots, t_{k}\right), t_{3}, \ldots, t_{k}\right) \equiv t_{0}\left(t_{3}, \ldots, t_{k}\right) .
\end{aligned}
$$

Then, if $g$ is nonpositive on the interval $-\infty<t_{2}<t_{0}\left(t_{3}, \ldots, t_{k}\right)$ for all $t_{3}, \ldots, t_{k}$ for which $g$ is defined, the maximum shortcoming of $\delta$ can only be attained on the edges of the parameter space, i.e. on the set $\Omega_{\text {restr }}$ defined by $(2.3 .3)$.

Proof:

Since the envelope invariant power function is constant on hyperspheres $\sum \mu_{i}^{2}=$ constant, the method of proof of lemma 2.3 .2 carries over to the present problem. The joint density (3.1.3) of $\underline{t}_{1}, \underline{t}_{2}, \ldots, \underline{t}_{k}$ looks very much like a jointly normal density if we disregard the integration with respect to the variable v. As we can perform all the operations of the proof of lemma 2.3.2 under this integral sign and the factors $\mathrm{v}^{\frac{1}{2}}$ arising from differentiation and partial integration cancel out in the final expression corresponding to (2.3.10), the desired result follows by similar arguments. Q.E.D. 
Hence the computation of the maximum shortcoming of a test satisfying the requirements of this lemma is relatively easy.

In chapter 2 we found that the LR test and FISHER's combination procedure are rather satisfactory combination methods in the case where the variances are known. FISHER's procedure cannot be applied to the present problem, because the statistics $\underline{t}_{1}, \underline{t}_{2}, \ldots, \underline{t}_{k}$ are not independent. However, the LR test can easily be obtained. Starting from the joint distribution of $\underline{x}_{1}, \underline{x}_{2}, \ldots, \underline{x}_{k}$ and $\underline{s}^{2}$ (with $\sigma^{2}>0$ ), we find that the LR test rejects $H$ if

$$
\left\{\sum_{i=1}^{k} \underline{x}_{i}^{2} I(0, \infty)\left(\underline{x}_{i}\right)\right\}\left\{\underline{s}^{2}+\sum_{i=1}^{k} \underline{x}_{i}^{2} I_{(-\infty, 0)}\left(\underline{x}_{i}\right)\right\}^{-1} \geq \tau_{\alpha}^{2} \text {, }
$$

or equivalently, if

$$
\left\{\sum_{i=1}^{k} \underline{t}_{i}^{2} I_{(0, \infty)}\left(\underline{t}_{i}\right)\right\}\left\{N+\sum_{i=1}^{k} \underline{t}_{i}^{2} I_{(-\infty, 0)}\left(\underline{t}_{i}\right)\right\}^{-1} \geq \tau_{\alpha}^{2},
$$

where $\tau_{\alpha}>0$ is a constant such that the size of the test is $\alpha$. We note that the LR test exists only for $\alpha<1-2^{-k}$, since the acceptance region $A_{L R, \alpha}$ in $t$-space of the test (3.1.15) strictly contains the negative orthant. In the positive orthant the region $A_{L R, \alpha}$ is bounded by a hypersphere with radius $\tau_{\alpha} \sqrt{ } \mathbb{N}$ and centre 0 .

As the test $(3.1 .15)$ is a function of $\underline{t}_{1}, t_{2}, \ldots, t_{k}$ only, it is invariant. In $\mathrm{z}$-space the acceptance region assumes the form

$$
A_{L R, \alpha}=\left\{z \mid \sum_{i=1}^{k} z_{i}^{2} I_{(0, \infty)}\left(z_{i}\right)<\tau^{2}\left(1+\tau_{\alpha}^{2}\right)^{-1}\right\}
$$

(cf.(2.4.10)), and since the region is convex and monotone, the LR test is an admissible invariant test by theorem 3.1.1. Moreover, the LR test satisfies the conditions of lemma 3.1 .2 and hence its maximum shortcoming is attained on the edges of the parameter space.

To determine the critical value $\tau_{\alpha}^{2}$ of the size- $\alpha$ LR test we consider (3.1.14). Denoting the test statistic in $(3.1 .14)$ by $t_{L R}$, we have under $H$

$$
\begin{aligned}
\alpha & =P\left(\underline{t}_{L R} \geq \tau_{\alpha}^{2}\right) \\
& =\sum_{j=1}^{k}\left({ }_{j}^{k}\right) P\left(\underline{t}_{L R} \geq \tau_{\alpha}^{2} \mid \underline{x}_{1}>0, \ldots, \underline{x}_{j}>0, \underline{x}_{j+1} \leq 0, \ldots, \underline{x}_{k} \leq 0\right) .
\end{aligned}
$$




$$
\begin{array}{r}
\cdot P\left(\underline{x}_{1}>0, \ldots, \underline{x}_{j}>0, \underline{x}_{j+1} \leq 0, \ldots, \underline{x}_{k} \leq 0\right) \\
=2^{-k} \sum_{j=1}^{k}\left(\begin{array}{l}
k \\
j
\end{array}\right) P\left(\underline{F}_{j, N+k-j} \geq \frac{N+k-j}{j} \tau_{\alpha}^{2}\right),
\end{array}
$$

where $\underline{F}_{j, N+k-j}$ has an $F-$ distribution with $j$ and $N+k-j$ degrees of freedom. With the aid of a computer $\tau_{\alpha}^{2}$ can easily be solved from equation (3.1.16).

Since $\underline{S}^{2} / N$ converges in probability to $\sigma^{2}$ for $N \rightarrow \infty$, the LR test appr ches for $N \rightarrow \infty$ the LR test in the case of known $\sigma^{2}$. In particular,

$$
\lim _{N \rightarrow \infty} N \tau_{\alpha}^{2}=\rho_{k, \alpha}^{2}
$$

where $\rho_{k, \alpha}^{2}$ is the critical value of the LR test in the case of unit variances (cf.(2.4.10)). In table 3.1.1 some values of $\mathrm{N} \tau_{\alpha}^{2}$ are shown.

We note that HEMELRIJK's suggestion (cf. section 1.5) to insert efficient estimators of the $\mu_{i}$ in the MP invariant test (3.1.6) again leads to the LR test if the $\mu_{i}$ are estimated by maximum likelihood.

In section 2.4 it was shown that the LR test for the problem of testing $H$ against $K$ with $\sigma^{2}$ known is asymptotically optimal for $\alpha \rightarrow 0$, i.e.its shortcoming tends uniformly to zero for $\alpha \rightarrow 0$. This property does not extend to the present problem with $\sigma^{2}$ unknown; on the contrary, for fixed $N$ and $k$ the maximum shortcoming of the LR test tends to one for $\alpha \rightarrow 0$ on each half-line through the origin in the parameter space. We shall not prove this assertion but a more general result showing that for sufficiently small values of $\alpha$ the maximum shortcoming of every combination procedure is close to one.

Theorem 3.1.2

For $\alpha \rightarrow 0$ and $N$ and $k$ fixed the maximum shortcoming (with respect to envelope invariant power) of the MS invariant size- $\alpha$ tests of $H$ against $K$ tends to one. Proof:

First we derive an asymptotic expression for $t_{N+k-1 ; \alpha}$ for $\alpha \rightarrow 0$. Omitting the index $\mathrm{N}+\mathrm{k}-1$, we find from the definition of the $t$-distribution

$$
\begin{aligned}
\alpha & =\left\{B\left(\frac{N+k}{2}, \frac{1}{2}\right)\right\}^{-1}(N+k-1)^{-\frac{1}{2}} \int_{t_{\alpha}}^{\infty}\left(1+\frac{t^{2}}{N+k-1}\right)^{-\frac{1}{2}(N+k)} d t \\
& =\left\{B\left(\frac{N+k}{2}, \frac{1}{2}\right)\right\}^{-1}(N+k-1)^{\frac{1}{2}(N+k-3)} t_{\alpha}^{-(N+k-1)}\left(1+C\left(t_{\alpha}^{-2}\right)\right)
\end{aligned}
$$




\begin{tabular}{|c|c|c|c|c|c|c|c|}
\hline $\mathrm{N}$ & .2 & .1 & .05 & .025 & .01 & .005 & .001 \\
\hline 2 & 2.240 & 5.414 & 11.434 & & & & \\
\hline 4 & 1.981 & 4.010 & 6.886 & 10.922 & 18.836 & 27.66 & 64.28 \\
\hline 6 & 1.896 & 3.623 & 5.839 & 8.641 & 13.489 & 18.259 & $34 \cdot 56$ \\
\hline 8 & 1.853 & 3.443 & 5.381 & 7.708 & 11.485 & 14.978 & 25.82 \\
\hline 10 & 1.827 & 3.339 & 5.126 & 7.203 & 10.451 & 13.343 & 21.82 \\
\hline 12 & 1.810 & 3.272 & 4.963 & 6.888 & 9.823 & 12.370 & 19.563 \\
\hline 15 & 1.793 & 3.205 & 4.806 & 6.589 & 9.240 & 11.484 & 17.590 \\
\hline 20 & 1.776 & 3.140 & 4.654 & 6.305 & 8.697 & 10.670 & 15.838 \\
\hline 30 & 1.759 & 3.076 & 4.508 & 6.036 & 8.194 & 9.929 & 14.307 \\
\hline$\infty$ & 1.725 & 2.952 & 4.231 & 5.537 & 7.289 & 8.628 & 11.763 \\
\hline
\end{tabular}

Table 3.1.1. Some values of $N \tau_{\alpha}^{2}$ for $\mathrm{k}=2$, where $\tau_{\alpha}^{2}$ is the critical value of the LR test (3.1.15). 
for $t_{\alpha} \rightarrow \infty$ (i.e. for $\left.\alpha \rightarrow 0\right)$, and hence

$(3.1 .17)$

$$
t_{\alpha}=(N+k-1)^{\frac{1}{2}} \gamma_{N, k} \alpha^{-1 /(N+k-1)}(1+o(1)) \text { for } \alpha \rightarrow 0,
$$

where $\gamma_{\mathrm{N}, \mathrm{k}}$ only depends on $\mathrm{N}$ and $\mathrm{k}$.

Consider a simple alternative $\mu^{(\alpha)}=\left(\mu_{1, \alpha}, 0, \ldots, 0\right)$ depending on $\alpha$. The MP invariant size- $\alpha$ test of $H$ against this alternative rejects $H$ if (cf.(3.1.6))

$$
\mu_{1, \alpha} \underline{x}_{1}\left(\underline{s}^{2}+\sum_{i=1}^{k} \underline{x}_{i}^{2}\right)^{-\frac{1}{2}} \geq d_{\alpha},
$$

or equivalently, for $\alpha<\frac{1}{2}$, if

$$
d_{\alpha}^{-2}\left(\mu_{1, \alpha}^{2}-d_{\alpha}^{2}\right) \underline{x}_{1}^{2}-\underline{s}^{2}-\sum_{i=2}^{k} \underline{x}_{i}^{2} \geq 0 \text { and } \underline{x}_{1}>0
$$

where by $(3.1 .7)$

$$
d_{\alpha}^{-2}\left(\mu_{1, \alpha}^{2}-d_{\alpha}^{2}\right)=(N+k-1) t_{\alpha}^{-2}
$$

Since under the alternative $\underline{x}_{1}$ has a normal $N\left(\mu_{1, \alpha}, 1\right)$ distribution, it follows that

$$
\lim _{\alpha \rightarrow 0} \beta_{\alpha, i n v}^{+}\left(\mu^{(\alpha)}\right)=1
$$

if and only if

$$
\lim _{\alpha \rightarrow 0} \mu_{1, \alpha} t_{\alpha}^{-1}=\infty,
$$

or equivalently, by $(3 \cdot 1.17)$, if and only if

$$
\lim _{\alpha \rightarrow 0} \mu_{1, \alpha} \alpha^{1 /(N+k-1)}=\infty .
$$

Also

$$
\lim _{\alpha \rightarrow 0} \beta_{\alpha, \text { inv }}^{+}\left(\mu^{(\alpha)}\right)=0
$$

if and only if

$$
\lim _{\alpha \rightarrow 0} \mu_{1, \alpha} \alpha^{1 /(N+k-1)}=0 .
$$

We recall that the critical region in t-space of the MP invariant size- $\alpha$ 
test against a simple alternative is one solid sheet of a $\mathrm{k}$-dimensional hyperbole of revolution, whose tangent half-cone has a semi-angle $\operatorname{arctn}\left\{(N+k-1)^{\frac{1}{2}} / t_{\alpha}\right\}$. Since $\operatorname{arctn} z=z+O\left(z^{3}\right)$ for $z \rightarrow 0$, we obtain from $(3.1 .17)$

$$
\operatorname{arctn}\left\{(N+k-1)^{\frac{1}{2}} / t_{\alpha}\right\}=\gamma_{N, k}^{-1} \alpha^{1 /(N+k-1)}(1+o(1)) \text { for } \alpha \rightarrow 0
$$

Hence, for sufficiently small $\alpha$, there exist at least

$$
m_{\alpha}=\operatorname{entier}\left\{\frac{\pi}{5} \gamma_{N, k} \alpha^{-1 /(N+k-1)}\right\}
$$

half-lines in the parameter space through the origin, $\mathrm{L}_{1, \alpha}, \ldots, \mathrm{I}_{\mathrm{m}_{\alpha}, \alpha}$ say, such that the critical regions in t-space of the MP invariant size- $\alpha$ tests against alternatives on these half-lines are disjoint. Let $T_{j, \alpha}$ be the critical region of the MP invariant size- $\alpha$ test against alternatives on $\mathrm{L}_{j, \alpha}\left(j=1,2, \ldots, \mathrm{m}_{\alpha}\right)$, and let $\mathrm{T}_{\mathrm{MS}, \alpha}$ denote the critical region of the MS invariant size- $\alpha$ test. Furthermore let $\tilde{\mathrm{T}}_{j, \alpha}=\mathrm{T}_{j, \alpha} \cap \mathrm{T}_{M S, \alpha}, j=1,2, \ldots, \mathrm{m}_{\alpha}$. Then there exists an index $j=j_{\alpha}$ such that

$$
P\left(\widetilde{T}_{j_{\alpha}, \alpha} \mid H\right)<\alpha / m_{\alpha} \text {. }
$$

Now choose an alternative $\bar{\mu}^{(\alpha)}$ on $\mathrm{L}_{j_{\alpha}, \alpha}$ whose coordinates satisfy

$$
\left(\sum_{i=1}^{k} \bar{\mu}_{i, \alpha}^{2}\right)^{\frac{1}{2}}=\left[\alpha^{1+1 /\{2(N+k-1)\}}\right]-1 /(N+k-1)
$$

From (3.1.23) we find

$$
\begin{aligned}
\mathrm{P}\left(\mathrm{T}_{\mathrm{MS}, \alpha} \mid \bar{\mu}^{-(\alpha)}\right) & =\mathrm{P}\left(\widetilde{\mathrm{T}}_{j_{\alpha}, \alpha} \mid \bar{\mu}(\alpha)\right)+\mathrm{P}\left(\mathrm{T}_{\mathrm{MS}, \alpha}-\mathrm{T}_{j_{\alpha}, \alpha} \mid \bar{\mu}^{-(\alpha)}\right) \\
< & P\left(\mathrm{~T}_{\alpha^{\prime}} \mid \bar{\mu}^{-(\alpha)}\right)+1-\mathrm{P}\left(\mathrm{T}_{j_{\alpha}, \alpha} \mid \bar{\mu}^{-(\alpha)}\right),
\end{aligned}
$$

where $\alpha^{\prime}=\alpha / m_{\alpha}$ and $T_{\alpha}$, is the critical region of the MP in variant size- $\alpha^{\prime}$ test against $\frac{\alpha}{\mu}(\alpha)$. As the envelope invariant power function is invariant under rotations in $\mu$-space, it follows from $(3.1 .18),(3.1 .19)$ and $(3.1 .24)$ that

$$
\lim _{\alpha \rightarrow 0} P\left(T_{j_{\alpha}, \alpha} \mid \bar{\mu}^{(\alpha)}\right)=\lim _{\alpha \rightarrow 0} \beta_{\alpha, \text { inv }}^{+}\left(\bar{\mu}^{(\alpha)}\right)=1
$$


and from $(3.1 .20),(3.1 .21)$ and $(3.1 .24)$ that

Hence

$$
\lim _{\alpha \rightarrow 0} P\left(\left.T_{\alpha^{\prime}}\right|^{-(\alpha)}\right)=\lim _{\alpha^{\prime} \rightarrow 0} \beta_{\alpha^{\prime}, \text { inv }}^{+}\left(\bar{\mu}^{-(\alpha)}\right)=0 .
$$

$$
\lim _{\alpha \rightarrow 0} R_{M S, \alpha}\left(\bar{\mu}^{-(\alpha)}\right)=\lim _{\alpha \rightarrow 0}\left[P\left(\left.T_{j_{\alpha}, \alpha}\right|^{-(\alpha)}\right)-P\left(T_{M S, \alpha} \mid \bar{\mu}^{-(\alpha)}\right)\right]=1 . \text { Q.E.D. }
$$

As a simple consequence of theorem 3.1.2 we find that the maximum shortcoming of every invariant size- $\alpha$ test tends to one for $\alpha \rightarrow 0$. Of course it is rather unrealistic to let $\alpha$ tend to zero for fixed $N$. If we let $N$ tend to infinity ( $\alpha$ fixed) the shortcoming of the MS invariant size- $\alpha$ tends to the shortcoming of the MS size- $\alpha$ test in the case where $\sigma^{2}$ is known and this shortcoming is uniformly small for small values of a (cf.section 2.4). Nevertheless theorem 3.1.2 indicates that the invariant combination procedures will have larger (maximum) shortcoming in the case where $\sigma^{2}$ is unknown than in the case where $\sigma^{2}$ is known.

We note that the MS invariant size- $\alpha$ tests of the theorem, which are MS relative to envelope invariant power, do not necessarily coincide with MS size- $\alpha$ tests of the original testing problem unrestricted by invariance (although there exist such tests which are invariant!), since these tests are MS with respect to different risk functions. However, as the shortcoming with respect to envelope power is larger than the shortcoming with respect to envelope invariant power for all invariant tests and all alternatives in the original parameter space, theorem 3.1.2 also holds for the shortcoming with respect to envelope power of the MS tests of the testing problem unrestricted by invariance.

The simplest invariant combination procedure for testing $\mathrm{H}$ against $\mathrm{K}$ is of course the symmetric linear procedure that rejects $H$ if

$$
\sum_{i=1}^{k} t_{i} \geq t_{N ; \alpha} \sqrt{k}
$$

This test is admissible by theorem 3.1 .1 and assumes its maximum shortcoming on the edges of the parameter space by lemma 3.1.2. Compared with the LR test it is more powerful for alternatives near the central half-line $\mu_{1}=\mu_{2}=\ldots=\mu_{k}>0$ and less powerful for alternatives near the edges of the parameter space, but not in such a marked degree as the MSSMP test (3.1.9). For $\mathbb{N}+\infty$ and fixed $\alpha$ the 
MSSMP test tends to the test (3.1.25).

Finally we mention the STUDENT t-test that arises if all the original observations in the $\mathrm{k}$ samples are pooled and $\sigma^{2}$ is estimated from the pooled sample. Obviously this test is the MP invariant test of $\mathrm{H}$ against alternatives with equal $\nu_{i}-\nu_{i}^{0}$, or equivalently, against alternatives where $\mu_{i}=\gamma \sqrt{n}, \gamma>0$ $(i=1,2, \ldots, k)$. Its maximum shortcoming heavily depends on the ratio's of the $\checkmark n_{i}$ and its power is relatively small for alternatives with widely different $v_{i}-v_{i}^{0}$. Hence this test should only be applied if prior information indicates that the $\nu_{i}-v_{i}^{0}$ are nearly equal.

Although numerical comparison of different invariant combination procedures is hardly feasible because of the complicated nature of the non-central distribution of $\underline{t}_{1}, \underline{t}_{2}, \ldots, \underline{t}_{k}$, we feel that if no prior information regarding the true means is available the LR test is the most adequate procedure for testing $\mathrm{H}$ against $\mathrm{K}$ since it gives the best protection against all alternatives simultaneously.

\subsection{OBSERVATIONS WITH UNEQUAL VARIANCES}

In this section it will be assumed that

$$
\mathrm{y}_{1,1}, \ldots, \mathrm{y}_{1, \mathrm{n}_{1}} ; \ldots ; \mathrm{y}_{\mathrm{k}, 1}, \ldots, \mathrm{y}_{\mathrm{k}, \mathrm{n}_{\mathrm{k}}}
$$

are $k$ samples of independent observations with normal $N\left(\nu_{i}, \sigma_{i}^{2}\right)$ distributions $(i=1,2, \ldots, k)$. Then

and

$$
y_{i}=n_{i}^{-1} \sum_{j=1}^{n_{i}} y_{i, j}, \quad i=1,2, \ldots, k,
$$

$$
\underline{s}_{i}^{2}=\sum_{j=1}^{n_{i}}\left(y_{i, j}-y_{i .}\right)^{2}, i=1,2, \ldots, k \text {, }
$$

constitute a set of sufficient statistics. Setting

$$
\begin{array}{ll}
\underline{x}_{i}=\left(\underline{y}_{i}-v_{i}^{0}\right) \sqrt{ } n_{i}, & i=1,2, \ldots, k, \\
\mu_{i}=\left(v_{i}-v_{i}^{0}\right) \sqrt{ } n_{i}, & i=1,2, \ldots, k, \\
m_{i}=n_{i}-1, & i=1,2, \ldots, k,
\end{array}
$$

the problem of testing $H^{*}$ against $\mathrm{K}^{*}$ may be formulated as follows. 
The random variables $\underline{x}_{1}, \underline{x}_{2}, \ldots, \underline{x}_{k}$ are independent and normally distributed with expectations $E \underline{x}_{i}=\mu_{i} \geq 0$ and variances $\sigma_{i}^{2}$ for $i=1,2, \ldots, k$, the random variables $\underline{s}_{1}^{2}, \underline{s}_{2}^{2}, \ldots, \underline{s}_{k}^{2}$ are independent of each other and of the $\underline{x}_{i}$ and are distributed as $\sigma_{i}^{2} \underline{x}_{m_{i}}^{2}(i=1,2, \ldots, k)$.

The hypothesis to be tested is

against

$$
H: \mu=\left(\mu_{1}, \ldots, \mu_{k}\right)=0, \quad \sigma_{i}^{2}>0(i=1,2, \ldots, k)
$$

$$
K: \mu \cdot \geq 0, \sigma_{i}^{2}>0(i=1,2, \ldots, k) .
$$

In applications this testing problem is of more importance than the problem discussed in the preceding section (with equal $\sigma_{i}^{2}$ ). Unfortunately it turns out that the present problem is essentially more difficult and our attempts to obtain theoretical properties of combination procedures were defeated. There are two reasons for these difficulties: the fact that we have $k$ (instead of one) nuisance parameters $\sigma_{1}^{2}, \ldots, \sigma_{\mathrm{k}}^{2}$ and the untractable form of the non-central t-distributions; the independence of the t-statistics is of little help.

The testing problem remains invariant under the group $G$ of scale transformations $g$

$$
\left(\underline{x}_{1}, \ldots, \underline{x}_{k}, \underline{s}_{1}, \ldots, \underline{s}_{k}\right) \stackrel{g}{\rightarrow}\left(c_{1} \underline{x}_{1}, \ldots, c_{k} \underline{x}_{k}, c_{1} \underline{s}_{1}, \ldots, c_{k} \underline{s}_{k}\right),
$$
where $c_{1}, \ldots, c_{k}$ are positive. A maximal invariant under $G$ is the set of independent t-statistics

$$
\underline{t}_{1}=m_{1}^{\frac{1}{2}} \underline{x}_{1} / \underline{s}_{1}, \cdots, t_{k}=m_{k}^{\frac{1}{2}} x_{k} / \underline{s}_{k}
$$

The power of any invariant test, i.e. any test based on $t_{1}, \ldots, t_{k}$, depends only on the parameters

$$
\mu_{1} / \sigma_{1}, \ldots, \mu_{k} / \sigma_{k}
$$

a maximal invariant under the induced group $\bar{G}$.

Restricting our attention to invariant tests, we may assume without loss of generality that $\sigma_{1}^{2}=\ldots=\sigma_{k}^{2}=1$. Each statistic $t_{i}$ has in the general case a non-central t-distribution with $m_{i}$ degrees of freedom; the density function is

$$
\begin{gathered}
p_{i}\left(t_{i} ; \mu_{i}\right)= \\
=\left\{2^{\frac{1}{2}\left(m_{i}+1\right)}\left(\pi m_{i}\right)^{\frac{1}{2}} \Gamma\left(\frac{1}{2} m_{i}\right)\right\}^{-1} \int_{0}^{\infty} v^{\frac{1}{2}\left(m_{i}-1\right)} \exp \left[-\frac{v}{2}-\frac{1}{2 m_{i}}\left(t_{i} v^{\frac{1}{2}}-\mu_{i} m_{i}^{\frac{1}{2}}\right)^{2}\right] d v \text {, }
\end{gathered}
$$


where the integral may also be written as

$$
\exp \left(-\frac{1}{2} \mu_{i}^{2}\right)\left(1+\frac{1}{m_{i}} t_{i}^{2}\right)^{-\frac{1}{2}\left(m_{i}+1\right)} \int_{0}^{\infty} v^{\frac{1}{2}\left(m_{i}-1\right)} \exp \left[-\frac{v}{2}+\left(m_{i}+t_{i}^{2}\right)^{-\frac{1}{2}} v^{\frac{1}{2}} \mu_{i} t_{i}\right] d v \text {. }
$$

Application of the NEYMAN-PEARSON lemma yields that the MP invariant size- $\alpha$ test of $\mathrm{H}$ against a simple alternative $\mu$ rejects $\mathrm{H}$ if

$$
\prod_{i=1}^{k} \int_{0}^{\infty} v_{i}^{\frac{1}{2}\left(m_{i}-1\right)} \exp \left[-\frac{v_{i}}{2}+\left(m_{i}+t_{i}^{2}\right)^{-\frac{1}{2}} v_{i}^{\frac{1}{2}} \mu_{i} t_{i}\right] d v \geq c_{\alpha}
$$

where $c_{\alpha}$ is a suitable constant. If $\mu_{i}>0$ and $\mu_{j}=0$ for $j \neq i$, this test reduces to the test

$$
\text { reject } H \text { if } t_{i} \geq t_{m_{i} ; \alpha} \text {. }
$$

But in general the critical value of the test (3.2.3) cannot easily be determined and hence it is virtually impossible to obtain the envelope invariant power function, except on the edges of the parameter space.

We have not been able to characterize the admissible invariant test of $\mathrm{H}$ against $\mathrm{K}$ (cf. theorem 3.1.1); the class of invariant tests with a.e. monotone acceptance regions in t-space is essentially complete by theorem 1.4.3, but this class is suspected to contain many inadmissible procedures.

The LR test of $\mathrm{H}$ against $\mathrm{K}$ is invariant and rejects $\mathrm{H}$ if

$$
\sum_{i=1}^{k}\left(m_{i}+1\right) \log \left\{1+m_{i}^{-1} t_{i}^{2} I_{(0, \infty)}\left(t_{i}\right)\right\} \geq c_{\alpha},
$$

where $c_{\alpha}$ is an appropriate constant. For not too small values of the $m_{i}$ this test is approximately equivalent to the test that rejects $H$ if

$$
\sum_{i=1}^{k} \frac{t_{i}^{2}}{I}(0, \infty)\left(t_{i}\right) \geq c_{\alpha}
$$

(cf.(2.4.10)). Although in principle the critical value of this test can be computed, this test does not have much practical value because $c_{\alpha}$ depends on $\mathrm{m}_{1}, \mathrm{~m}_{2}, \ldots, \mathrm{m}_{\mathrm{k}}$ and $\alpha$ and extensive tables would be necessary.

Linear combination of the $t_{i}$ gives rise to the same difficulties; computation of the critical values is again a tedious affair since the convolution of (central) t-distributions does not have a nice form.

Hence it appears that FISHER's combination procedure applied to $\underline{t}_{1}, t_{2}, \ldots, t_{k}$ 
is the only exact combination method that is readily available. The results of chapter 2 suggest that this might be a reasonable solution to the combination problem.

In the large sample case we have of course more possibilities; symmetric linear combination of the $t_{i}$ and the (approximate) LR test (3.2.5) with $c_{\alpha} \simeq \rho_{k, \alpha}^{2}$ are then competitors of FISHER's procedure. 
CHAPTER 4. RELATED ASYMPTOTIC RESULTS

\subsection{A GENERAL ASYMPTOTIC APPROACH}

In the preceding chapters we studied two asymptotic approaches to combination problems.

In the first place we reduced quite general combination problems to the simpler problem of combining $\mathrm{k}$ independent normal random variables with unit variances. This was accomplished by letting the sample sizes tend to infinity for a fixed significance level $\alpha$ and relying on the asymptotic normality of many test statistics (section 2.1).

Secondly we studied in section 2.4 and section 3.1 the shortcoming of test procedures for the normal combination problem and the combination of t-tests respectively if the significance level a tends to zero. In the normal case we found that the shortcoming of the LR test tends uniformly to zero for $\alpha \rightarrow 0$. If we have $\mathrm{k}$ samples from normal distributions with known variances (cf. example 2.5.1), this property of the LR test continues to hold. In some other combination problems the maximum shortcoming of the LR test also tends to zero for $\alpha \rightarrow 0$, and fixed sample sizes. As an example we mention the combination of $\mathrm{k}$ tests of the hypotheses $\beta_{i}=\beta_{i}^{0}$ against $\beta_{i}<\beta_{i}^{0}(i=1,2, \ldots, k)$, where the $\beta_{i}$ are the scale parameters of gamma distributions with known shape parameters and sample of size $n_{i}$ from each of the $\mathrm{k}$ populations are available (cf. example 2.2.3). However, it was shown in chapter 3 (theorem 3.1.2) that if one-sample t-tests are to be combined, the maximum shortcoming of the LR combination procedure tends to one for $\alpha \rightarrow 0$ and fixed sample sizes.

However, it does not seem to be very realistic to let $\alpha$ tend to zero for fixed sample sizes $n_{i}(i=1,2, \ldots, k)$. It is more natural to let the sample sizes tend to infinity and the significance level to zero simultaneously. In this setup both the probabilties of errors of the first and second kind tend to zero. Moreover, it is then possible to study the perormance of test procedures for nonlocal alternatives, as opposed to the classical NEYMAN-PEARSON approach which provides information on the power functions for near alternatives only. of course one has to decide on the choice of $\alpha$ as a function of the sample sizes and this 
may be a difficult decision since simple rules of thumb are not (yet) available.

One would expect that if the test statistics are asymptotically normal and the rate of convergence of $\alpha$ to zero as a function of the sample sizes is relatively slow, then the maximum shortcoming of the LR combination procedure will tend to zero as in the normal case. However, if the rate of convergence of $\alpha$ to zero is fast, the extreme tails of the distributions become essential and here the asymptotic normality breaks down. A different analysis is then necessary.

In fact, W. HOEFFDING ([23],[24]) used this general asymptotic approach to study a quite different testing problem. He investigated the performance of the LR procedure for testing a hypothesis in multinomial distributions against general alternatives. He found that if the significance level $\alpha_{N}$ tends fast to zero as a function of the sample size $\mathbb{N}$, the LR test is considerably more powerful than any other test which is sufficiently different from the LR test. For a more precise formulation we refer to [23] (cf. also[22] and [43]). In section 4.2 it will be shown that the maximum shortcoming of the LR test of a simple hypothesis in a multinomial distribution tends to zero if $\alpha_{N}$ tends to zero slowly for $N \rightarrow \infty$. This property is based on the fact that the multinomial distribution is asymptotically normal for $N \rightarrow \infty$.

The same kind of argument that we shall use to prove this result can also be applied to show that the LR procedure for combining $\mathrm{k}$ binomial tests of the hypotheses $p_{i}=p_{i}^{0}$ against $p_{i}>p_{i}^{0}(i=1,2, \ldots, k)$ has uniformly vanishing shortcoming if the sample sizes tend to infinity and $\alpha$ tends slowly to zero as a function of the sample sizes. The proof of this property is more or less parallell to the proof of theorem 4.2.1 for multinomial distributions and is omitted because of its technical character.

The study of the LR test in multinomial distributions is motivated by our feeling that in this case the LR test has a uniformly vanishing shortcoming for $\alpha_{N} \rightarrow 0$ not only if $\alpha_{N}$ converges slowly but also if $\alpha_{N}$ tends fast to zero. Further work on this subject is in progress.

\subsection{THE LIKELIHOOD RATIO TEST IN MULTINOMIAL DISTRIBUTIONS}

Let $\underline{x}_{1}, \underline{x}_{2}, \ldots, \underline{x}_{k}$ have a multinomial distribution with parameters $p_{1}, p_{2}, \ldots, p_{k}$, 


$$
\mathrm{P}\left(\underline{\mathrm{x}}_{1}=\mathrm{x}_{1}, \ldots, \underline{\mathrm{x}}_{\mathrm{k}}=\mathrm{x}_{\mathrm{k}}\right)=\frac{\mathrm{N} !}{\mathrm{x}_{1} ! \ldots \mathrm{x}_{\mathrm{k}} !} \mathrm{p}_{1}{ }^{\mathrm{x}_{1}} \ldots \mathrm{p}_{\mathrm{k}} \mathrm{x}^{\mathrm{x}},
$$

where $\mathbb{N}$ is the sample size, $x_{1}+x_{2}+\ldots+x_{k}=N$ and $p=\left(P_{1}, \ldots, p_{k}\right)$ is any point in the simplex

$$
\Omega=\left\{\left(\mathrm{y}_{1}, \ldots, \mathrm{y}_{\mathrm{k}}\right) \mid \sum_{i=1}^{\mathrm{k}} \mathrm{y}_{\mathrm{i}}=1, \mathrm{y}_{\mathrm{i}} \geq 0 \text { for } \mathrm{i}=1,2, \ldots, \mathrm{k}\right\} .
$$

Let $\mathrm{p}^{0}$ be an arbitrary point of the set

$$
\Omega^{*}=\left\{\left(y_{1}, \ldots, y_{k}\right) \mid \sum_{i=1}^{k} y_{i}=1, y_{i}>0 \text { for } i=1,2, \ldots, k\right\} .
$$

We consider tests of the simple hypothesis

$$
H: p=p^{0} \in \Omega^{*}
$$

against the composite alternative

$$
K: p \in \Omega-\left\{p^{0}\right\}
$$

It is assumed that the significance level $\alpha_{N}$ depends on $N$ in such a way that $\alpha_{N} \rightarrow 0$ for $N \rightarrow \infty$.

The size- $\alpha_{N}$ LR test of $H$ against $K$ rejects $H$ if

$$
\sum_{i=1}^{k} \underline{x}_{i} \log \left(\underline{x}_{i} /\left(N p_{i}^{0}\right)\right) \geq c_{N},
$$

possibly with randomization on the boundary of the critical region. The MP size- $\alpha_{N}$ test of $\mathrm{H}$ against a simple alternative $p=p^{\prime} \epsilon \Omega^{*}-\left\{p^{0}\right\}$ rejects $\mathrm{H}$ if

$$
\sum_{i=1}^{k} \underline{x}_{i} \log \left(p_{i}^{!} / p_{i}^{0}\right) \geq a_{N}
$$

possibly with randomization on the boundary of the critical region. The critical value $d_{N}$ depends not only on $N$ but also on the alternatives $p^{\prime}$ considered.

Let $\beta_{N}^{+}(p)$ and $\beta_{L R, N}(p)$ denote the envelope power function and the power function of the LR test respectively for a given $N$ (we omit the subscript $\alpha_{N}$ because it is a function of $N$ ). The shortcoming of the LR test for a given $N$ is then denoted by 


$$
R_{L R, N}(p)=\beta_{N}^{+}(p)-\beta_{L R, N}(p) .
$$

We shall often make use of the notation $v_{N} \sim w_{N}$, meaning that $v_{N} / w_{N} \rightarrow 1$ for $N \rightarrow \infty$. In the sequel the alternatives considered will usually depend on $N$. Nevertheless we shall often write $p^{\prime}$ instead of $p^{\prime}(\mathbb{N})$.

First we determine the size- $\alpha_{N}$ envelope power function for values of $\alpha_{N}$ converging slowly to zero.

\section{Lemma 4.2 .1}

Let $\rho$ be any fixed positive function of $N$ satisfying

$(4.2 .7) \quad \lim _{N \rightarrow \infty} N_{\rho}^{\gamma}(N)=0$ for some $\gamma>0$

and

(4.2.8) $\quad \lim _{\mathbb{N} \rightarrow \infty} N^{1 / 6} \rho(\mathbb{N})=\infty$.

Then, if

$(4.2 .9)$

$$
u_{\alpha_{N}}=N^{1 / 6} \rho(N),
$$

we have

$(4.2 .10)$

$$
\beta_{N}^{+}\left(p^{\prime}\right)=1-\Phi\left(u_{\alpha_{N}}-N^{\frac{1}{2}}\left[\sum_{i=1}^{k} \frac{\left(p_{i}^{\prime}-p_{i}^{0}\right)^{2}}{p_{i}^{0}}\right]^{\frac{1}{2}}\right)+\sigma(1)
$$

for $N \rightarrow \infty$ uniformly for all $p^{\prime} \in \Omega$.

Proof:

Let

$$
\text { (4.2.11) } \varepsilon=\frac{1}{2} \min _{\substack{1 \leq i \leq k \\ i}} p_{i}^{0} \text {; }
$$

since $p_{i}^{0}>0(i=1,2, \ldots, k)$ we also have $\varepsilon>0$. For each $N$ we divide the parameter space $\Omega$ into three parts:

$$
\begin{aligned}
& \Omega_{N}^{(1)}=\left\{p^{\prime} \mid \min _{i} p_{i}^{\prime}<\varepsilon\right\}, \\
& \Omega_{N}^{(2)}=\left\{p^{\prime}\left|\max _{i}\right| p_{i}^{\prime}-p_{i}^{0} \mid \geq N^{-1 / 6}, \min _{i} p_{i}^{\prime} \geq \varepsilon\right\}, \\
& \Omega_{N}^{(3)}=\left\{p^{\prime}\left|\max _{i}\right| p_{2}^{\prime}-p_{i}^{0} \mid<N^{-1 / 6}, \min _{i} p_{i}^{\prime} \geq \varepsilon\right\} .
\end{aligned}
$$

We shall prove uniform convergence for each of these three subspaces. 
First we assume that $\mathrm{p}^{\prime} \geq \varepsilon$, i.e. $\mathrm{p}^{\prime} \in\left(\Omega_{\mathbb{N}}^{(2)} \cup \Omega_{\mathbb{N}}^{(3)}\right)$. We denote the test statistic of the MP test of $H$ against $p^{\prime}$ by

$$
\underline{t}^{\prime}=\sum_{i=1}^{k} \underline{x}_{i} \log \left(p_{i}^{\prime} / p_{i}^{0}\right) \text {. }
$$

For general $\mathrm{p}$ this statistic is the sum of $\mathrm{N}$ independent identically distributed random variables $\underline{a}_{j}(j=1,2, \ldots, N)$, where the distribution of $\underline{a}_{j}$ is given by

\section{Hence}

$$
P\left(\underline{a}_{j}=\log \left(p_{i}^{!} / p_{i}^{0}\right)\right)=p_{i} \quad, i=1,2, \ldots, k \text {. }
$$

$$
E\left(\underline{t}^{\prime} \mid p\right)=N \sum_{i=1}^{k} p_{i} \log \left(p_{i}^{\prime} / p_{i}^{0}\right)
$$

and

$$
\sigma^{2}\left(\underline{t}^{\prime} \mid p\right)=N\left\{\sum_{i=1}^{k} p_{i} \log ^{2}\left(p_{i}^{\prime} / p_{i}^{0}\right)-\left[\sum_{i=1}^{k} p_{i} \log \left(p_{i}^{\prime} / p_{i}^{0}\right)\right]^{2}\right\} .
$$

Moreover, the central limit theorem implies that

$$
P\left(\underline{t}^{\prime} \geq \alpha_{N} \mid p\right)-1+\Phi\left(\frac{d_{N}-E\left(\underline{t}^{\prime} \mid p\right)}{\sigma\left(\underline{t}^{\prime} \mid p\right)}\right) \rightarrow 0 \text { for } N \rightarrow \infty
$$

uniformly for all $d_{N}$ and all $p^{\prime} \geq \varepsilon, p \geq \varepsilon$. The uniformity in $p^{\prime}$ and $p$ is a consequence of the fact that the characteristic function of $\left\{\underline{t}^{\prime}-E\left(\underline{t}^{\prime} \mid p\right)\right\} / \sigma\left(\underline{t}^{\prime} \mid p\right)$ tends to the characteristic function of the standard normal distribution uniformly for all $\mathrm{p}^{\prime} \geq \varepsilon$ and all $\mathrm{p} \geq \varepsilon$.

From theorem 3 of YU.V.LINNIK $([33], I)$ we derive that

$$
\alpha_{N}=P\left(\underline{t}^{\prime} \geq \alpha_{N} \mid p^{0}\right) \sim 1-\Phi\left(\frac{d_{N}-E\left(\underline{t}^{\prime} \mid p^{0}\right)}{\sigma\left(\underline{t}^{\prime} \mid p^{0}\right)}\right) \text { for } N \rightarrow \infty
$$

uniformly for all $\mathrm{d}_{\mathrm{N}}$ and $\mathrm{p}^{\prime} \geq \varepsilon$ satisfying

$$
\left|\frac{d_{N}-E\left(\underline{t}^{\prime} \mid p^{0}\right)}{\sigma\left(\underline{t}^{\prime} \mid p^{0}\right)}\right| \leq N^{1 / 6} \rho(N) \text {. }
$$

Hence, if $u_{\alpha_{N}}$ is given by (4.2.9), we find that under $H$

$$
\frac{d_{N}-E\left(\underline{t}^{\prime} \mid p^{0}\right)}{\sigma\left(\underline{t}^{\prime} \mid p^{0}\right)}=u_{\alpha_{N}}\left(1+\sigma\left(u_{\alpha_{N}}^{-2}\right)\right) \quad \text { for } N \rightarrow \infty,
$$


or, by $(4.2 .12)$ and $(4.2 .13)$,

$$
\begin{aligned}
\alpha_{N} & =N^{\frac{1}{2}}\left\{\sum_{i} p_{i}^{0} \log \frac{p_{i}^{!}}{p_{i}^{0}}-\left[\sum_{i} p_{i}^{0} \log \frac{p_{i}^{!}}{p_{i}^{0}}\right]^{2}\right\}^{\frac{1}{2}} u_{\alpha_{N}}\left(1+\sigma\left(u_{\alpha_{N}}^{-2}\right)\right)+ \\
& +N \sum_{i} p_{i}^{0} \log \frac{p_{i}^{0}}{p_{i}^{0}},
\end{aligned}
$$

where $o\left(u_{\alpha_{N}}^{-2}\right)$ does not depend on the particular choice of $p^{\prime}$. From (4.2.14) we obtain that ${ }^{N}$ under the alternative $p=p^{\prime}$

(4.2.17) $\quad P\left(\underline{t}^{\prime} \geq \alpha_{N} \mid p^{\prime}\right)=1-\Phi\left(\frac{\alpha_{N}-E\left(\underline{t}^{\prime} \mid p^{\prime}\right)}{\sigma\left(\underline{t}^{\prime} \mid p^{\prime}\right)}\right)+\sigma(1)$ for $N \rightarrow \infty$

uniformly for all $p^{\prime} \geq \varepsilon$, where $a_{N}$ is given by (4.2.16). By $(4.2 .12)$ and $(4.2 .13)$ the argument of $\Phi$ in the right-hand member is equal to

$$
\frac{\left\{\sum_{i}^{0} p_{i}^{0} \log ^{2} \frac{p_{i}^{!}}{p_{i}^{0}}-\left[\sum_{i}^{0} p_{i}^{0} \log \frac{p_{i}^{\prime}}{p_{i}^{0}}\right]^{2}\right\}^{\frac{1}{2}} u_{\alpha_{N}}\left(1+\sigma\left(u_{\alpha_{N}^{-1}}^{-1}\right)\right)-N^{\frac{1}{2}} \sum_{i}\left(p_{i}^{!}-p_{i}^{0}\right) \log \frac{p_{i}^{!}}{p_{i}^{0}}}{\left\{\sum_{i} p_{i}^{!} \log ^{2} \frac{p_{i}^{\prime}}{p_{i}^{0}}-\left[\sum_{i} p_{i}^{\prime} \log \frac{p_{i}^{\prime}}{p_{i}^{0}}\right]^{2}\right\}^{\frac{1}{2}}}
$$

This expression tends uniformly to $-\infty$ for $N \rightarrow \infty$ and all $p^{\prime} \in \Omega_{N}^{(2)}$.

It follows from $(4.2 .17)$ that in this case $(4.2 .10)$ is uniformly satisfied. Now let us assume that $p^{\prime} \in \Omega_{\mathbb{N}}^{(3)}$ and define

$$
\zeta_{\mathrm{N}}=\max _{1 \leq i \leq k}\left|p_{i}^{\prime}-p_{i}^{0}\right| ;
$$

then $0 \leq \zeta_{N} \leq N^{-1 / 6}$. By expansion of the logarithm it is easily shown that for $N \rightarrow \infty$

$(4.2 .19)$

$$
\begin{aligned}
& \sum_{i} p_{i}^{0} \log ^{2} \frac{p_{i}^{\prime}}{p_{i}^{0}}=\sum_{i} \frac{\left(p_{i}^{!}-p_{i}^{0}\right)^{2}}{p_{i}^{0}}+\sigma\left(\zeta_{N}^{3}\right), \\
& {\left[\sum_{i} p_{i}^{0} \log \frac{p_{i}^{\prime}}{p_{i}^{0}}\right]^{2}=\sigma\left(\zeta_{N}^{4}\right),} \\
& \sum_{i} p_{i}^{!} \log \frac{p_{i}^{0}}{p_{i}^{0}}=\frac{1}{2} \sum_{i} \frac{\left(p_{i}^{!}-p_{i}^{0}\right)^{2}}{p_{i}^{0}}+\sigma\left(\zeta_{N}^{3}\right), \\
& \sum_{i}\left(p_{i}^{\prime}-p_{i}^{0}\right) \log \frac{p_{i}^{\prime}}{p_{i}^{0}}=\sum_{i} \frac{\left(p_{i}^{!}-p_{i}^{0}\right)^{2}}{p_{i}^{0}}+\sigma\left(\zeta_{N}^{3}\right),
\end{aligned}
$$




$$
\begin{aligned}
& \sum_{i} p_{i}^{\prime} \log ^{2} \frac{p_{i}^{\prime}}{p_{i}^{0}}=\sum_{i} \frac{\left(p_{i}^{\prime}-p_{i}^{0}\right)^{2}}{p_{i}^{0}}+\sigma\left(\zeta_{N}^{3}\right), \\
& {\left[\sum_{i} p_{i}^{\prime} \log \frac{p_{i}^{\prime}}{p_{i}^{0}}\right]^{2}=\sigma\left(\zeta_{N}^{4}\right) .}
\end{aligned}
$$

Substitution of these relations yields that the argument of $\Phi$ in the right-hand member of $(4.2 .17)$ is equal to

$$
\left\{u_{\alpha_{N}}-N^{\frac{1}{2}}\left[\sum_{i} \frac{\left(p_{i}-p_{i}^{0}\right)^{2}}{p_{i}^{0}}\right]^{\frac{1}{2}}\right\} \quad\left(1+\sigma\left(\zeta_{N}\right)\right)\left(1+\sigma\left(u_{\alpha_{N}}^{-2}\right)\right)
$$

for $N \rightarrow \infty$, and hence $(4 \cdot 2 \cdot 10)$ is uniformly satisfied in this case also.

It remains to consider the case where $p^{\prime} \in \Omega_{N}^{(1)}$. Without loss of generality we assume that $p_{1}^{1}<\varepsilon$. Hence by definition $p_{i}^{\prime}<\frac{1}{2} p_{1}^{0}$. Consider the test $\delta_{N}$ that rejects $H$ for small values of $\underline{x}_{1}$. By the same line of argument that we used before its critical value $\mathrm{g}_{\mathrm{N}}$ is equal to

$$
\mathrm{g}_{\mathrm{N}}=\mathrm{Np}_{1}^{0}+\mathrm{N}^{\frac{1}{2}}\left[\mathrm{p}_{1}^{0}\left(1-\mathrm{p}_{1}^{0}\right)\right]^{\frac{1}{2}} \mathrm{u}_{\alpha_{\mathrm{N}}}\left(1+\sigma\left(\mathrm{u}_{\alpha}^{-2}\right)\right) \text {, }
$$

provided (4.2.9) is satisfied, and its power against $\mathrm{p}^{\prime}$ is equal to

$$
1-\Phi\left(\frac{g_{N}-N p_{1}^{\prime}}{N^{\frac{1}{2}}\left\{p_{1}^{\prime}\left(1-p_{1}^{\prime}\right)\right\}^{\frac{1}{2}}}\right)+\sigma(1) \text { for } N \rightarrow \infty,
$$

where $\sigma\left(1\right.$ ) does not depend on $p_{1}^{\prime}$ (if $p_{1}^{\prime}=0$, the power is obviously equal to $1+\sigma(1)$. It is easily verified that the argument of $\Phi$ tends uniformly to - $\infty$ for $p_{1}^{\prime}<\varepsilon$, and hence the power of $\delta_{N}$ tends uniformly to one for all $p^{\prime} \in \Omega_{N}^{(1)}$. Since the MP test against $p^{\prime}$ has a power which is at least equal to that of $\delta_{N}$, it follows that the power of the MP test also converges uniformly to one for all $p^{\prime} \in \Omega_{N}^{(1)}$, in accordance with (4.2.10), and the proof of the lemma is complete.

Q.E.D.

Next we study the power of the size- $\alpha_{N}$ LR test for $\alpha_{\mathbb{N}}$ tending slowly to zero. To this end we need an asymptotic expression for the critical value of the size $-\alpha_{N}$ LR test $(4.2 .4)$

Lemma 4.2 .2

Let $x_{k-1}^{2} ; \alpha_{N}$ denote the upper $\alpha_{N}$-point of the chi-square distribution with $k-1$ 
degrees of freedom. Then, if the condition

$$
\chi_{k-1 ; \alpha_{N}}^{2}=\sigma\left(N^{1 / 6}\right) \quad \text { for } N \rightarrow \infty
$$

is satisfied, we have

$$
c_{N}=\frac{1}{2} \quad x_{k-1 ; \alpha_{N}}^{2}+\sigma(1) \quad \text { for } N \rightarrow \infty
$$

Proof:

Throughout the proof we assume that the hypothesis H holds true. Since

$$
\begin{aligned}
\log \frac{\underline{x}_{i}}{N p_{i}^{0}} & =\log \left(1+\frac{\underline{x}_{i}-N p_{i}^{0}}{N p_{i}^{0}}\right)= \\
& =\frac{\underline{x}_{i}-N p_{i}^{0}}{N p_{i}^{0}}-\frac{1}{2} \frac{\left(\underline{x}_{i}-N p_{i}^{0}\right)^{2}}{\left(N p_{i}^{0}\right)^{2}}+\sigma_{P}\left(N^{-3 / 2}\right)
\end{aligned}
$$

for $N \rightarrow \infty$, the test statistic of the LR test can be written in the form

$$
\begin{aligned}
\sum_{i=1}^{k} \underline{x}_{i} \log \frac{\underline{x}_{i}}{N p_{i}^{0}} & =\sum_{i=1}^{k}\left(\underline{x}_{i}-N p_{i}^{0}\right) \log \frac{\underline{x}_{i}}{N p_{i}^{0}}+N \sum_{i=1}^{k} p_{i}^{0} \log \frac{\underline{x}_{i}}{N p_{i}^{0}}= \\
& =\frac{1}{2} \sum_{i=1}^{k} \frac{\left(\underline{x}_{i}-N p_{i}^{0}\right)^{2}}{N p_{i}^{0}}+\sigma_{P}\left(N^{-\frac{1}{2}}\right) .
\end{aligned}
$$

It is well-known (cf. W. RICHTER [41]) that for $N \rightarrow \infty$

$$
P\left(\sum_{i=1}^{k} \frac{\left(\underline{x}_{i}-N p_{i}^{0}\right)^{2}}{N p_{i}^{0}}>a_{N}\right) \sim P\left(\underline{x}_{k-1}^{2}>a_{N}\right)
$$

if $a_{N}=v\left(N^{1 / 6}\right)$. Moreover, it can be shown as in section 20.6 of $\mathrm{H}$. CRAMER [8] that

$$
P\left(\frac{1}{2} \sum_{i=1}^{k} \frac{\left(\underline{x}_{i}-N p_{i}^{0}\right)^{2}}{N p_{i}^{0}}+\sigma_{p}\left(N^{-\frac{1}{2}}\right)>c_{N}\right) \sim P\left(\frac{1}{2} \sum_{i=1}^{k} \frac{\left(\underline{x}_{i}-N p_{i}^{0}\right)^{2}}{N p_{i}^{0}}>c_{N}\right)
$$

if $c_{N}=\sigma\left(N^{1 / 6}\right)$. The assertion of the lemma is now an immediate consequence of assumption $(4.2 .20)$ and $(4.2 .22)$

Lemma 4.2 .3

Let $\tau$ be any fixed positive function of $\mathbb{N}$ satisfying 


$$
\lim _{\mathbb{N} \rightarrow \infty} \tau(N)=0, \lim _{N \rightarrow \infty} N^{1 / 6} \tau(N)=\infty .
$$

Then, if

$(4.2 .23)$

$$
\chi_{k-1 ; \alpha_{N}}^{2}=N^{1 / 6} \tau(N)
$$

we have

$(4.2 .24)$

$$
\beta_{L R, N}\left(p^{\prime}\right)=1-\Phi\left(\frac{\chi_{k-1 ; \alpha}^{2}-N \sum_{i=1}^{k} \frac{\left(p_{i}^{!}-p_{i}^{0}\right)^{2}}{p_{i}^{0}}}{2 N^{\frac{1}{2}}\left[\sum_{i=1}^{k} \frac{\left(p_{i}^{\prime}-p_{i}^{0}\right)^{2}}{p_{i}^{0}}\right]^{\frac{1}{2}}}\right)+\sigma(1)
$$

for $\mathrm{N} \rightarrow \infty$ uniformly for all $\mathrm{p}^{\prime} \epsilon \Omega$.

\section{Proof:}

We start by remarking that from $(2.4 .13)$ and (2.4.15)

$(4.2 .25)$

$$
u_{\alpha_{N}}=\left(x_{k-1 ; \alpha_{N}}^{2}\right)^{\frac{1}{2}}+o(1) \quad \text { for } N \rightarrow \infty \text {. }
$$

Let $\varepsilon$ again be defined by (4.2.11)and let $\mathrm{p}^{\prime}$ denote the true parameter value. For each $N$ we divide the parameter space $\Omega$ into four parts:

$$
\begin{aligned}
& \Omega_{N}^{(1)}=\left\{p^{\prime} \mid \min _{i} p_{i}^{\prime}<\varepsilon\right\}, \\
& \Omega_{N}^{(2)}=\left\{p^{\prime}\left|\max _{i}\right| p_{j}^{\prime}-p_{j}^{0} \mid>N^{-1 / 5}, \underset{i}{\min } p_{i}^{\prime} \geq \varepsilon\right\}, \\
& \Omega_{N}^{(3)}=\left\{p^{\prime}\left|\max _{i}\right| p_{j}^{\prime}-p_{j}^{0} \mid \leq(\varepsilon / k)^{\frac{1}{2}} N^{-5 / 12} \tau^{\frac{1}{2}}(N), \quad \underset{i}{\min } p_{i}^{\prime} \geq \varepsilon\right\}, \\
& \Omega_{N}^{(4)}=\left\{p^{\prime}\left|(\varepsilon / k)^{\frac{1}{2}} N^{-5 / 12} \tau^{\frac{1}{2}}(N)<\max _{i}\right| p_{j}^{\prime}-p_{j}^{0} \mid \leq N^{-1 / 5}\right. \text {, } \\
& \left.\min _{i} p_{i}^{\prime} \geq \varepsilon\right\}
\end{aligned}
$$

We shall again prove uniform convergence for each of these subspaces.

First we assume that $\mathrm{p}^{\prime} \geq \varepsilon$. Since

$$
\log \frac{\underline{x}_{i}}{N p_{i}^{0}}=\log \frac{p_{i}^{\prime}}{p_{i}^{0}}+\log \frac{\underline{x}_{i}}{N p_{i}}=\log \frac{p_{i}^{\prime}}{p_{i}^{0}}+\frac{\frac{x_{i}}{N}-N p_{i}^{\prime}}{N p_{i}}-\frac{1}{2} \frac{\left(\underline{x}_{i}-N p_{i}\right)^{2}}{\left(N p_{i}\right)^{2}}+\sigma_{P}\left(N^{-3 / 2}\right)
$$


for $N \rightarrow \infty$ uniformly for $p^{\prime} \geq \varepsilon$, the test statistic $\underline{t}^{*}$ of the LR test can be written in the form

$$
\begin{aligned}
\underline{t}^{*}= & \sum_{i} \underline{x}_{i} \log \frac{\underline{x}_{i}}{N p_{i}^{0}}=\sum_{i}\left(\underline{x}_{i}-N p_{i}^{\prime}\right) \log \frac{\underline{x}_{i}}{N p_{i}^{0}}+N \sum_{i} p_{i} \log \frac{\frac{x_{i}}{N p_{i}^{0}}}{N p_{i}} \\
= & \sum_{i}\left(\underline{x}_{i}-N p_{i}^{\prime}\right) \log \frac{p_{i}^{\prime}}{p_{i}^{0}}+\frac{1}{2} \sum_{i} \frac{\left(\underline{x}_{i}-N p_{i}\right)^{2}}{N p_{i}}+N \sum_{i} p_{i} \log \frac{p_{i}^{!}}{p_{i}^{0}}+ \\
& +\sigma_{P}\left(N^{-\frac{1}{2}}\right) .
\end{aligned}
$$

By writing

$$
\sum_{i=1}^{k} p_{i}^{\prime} \log \frac{p_{i}^{!}}{p_{i}^{0}}=p_{j}^{\prime} \log \frac{p_{i}^{\prime}}{p_{j}^{0}}+\sum_{i \neq j} p_{i}^{\prime} \log \frac{p_{i}^{\prime}}{p_{i}^{0}} \quad(j \in\{1,2, \ldots, k\})
$$

and minimizing the last expression in the right-hand member for fixed $p_{j}^{\prime}$ and $p^{0}$ with the aid of Lagrange multipliers ( the stationary point is a minimum because the function $\left[p_{i}^{\prime} \log \left(p_{i}^{\prime} / p_{i}^{0}\right)\right.$ is convex in $p^{\prime}$ on $\left.\Omega\right)$, we find

$$
\sum_{i=1}^{k} p_{i}^{\prime} \log \frac{p_{i}^{\prime}}{p_{i}^{0}} \geq p_{j}^{\prime} \log \frac{p_{j}^{\prime}}{p_{j}^{0}}+\left(1-p_{j}^{\prime}\right) \log \frac{1-p_{j}^{\prime}}{1-p_{j}^{0}}
$$

for any $j \in\{1,2, \ldots, k\}$. It is now easily verified that

$$
\sum_{i=1}^{k} p_{i}^{\prime} \log \frac{p_{i}^{!}}{p_{i}^{0}} \geq \frac{1}{2}\left(p_{j}^{\prime}-p_{j}^{0}\right) \log \left(1+\frac{1}{2} \frac{p_{i}^{\prime}-p_{j}^{0}}{p_{j}^{0}}\right) .
$$

Hence, if $p^{\prime} \in \Omega_{N}^{(2)}$, we find that

$$
\sum_{i=1}^{k} p_{i}^{!} \log \frac{p_{i}^{\prime}}{p_{i}^{0}}>\frac{1}{8} \max _{i}\left(p_{i}-p_{i}^{0}\right)^{2}>\frac{1}{8} N^{-2 / 5}
$$

for all suffiently large $N$ (independent of $\mathrm{p}^{\prime}$ ) and the third term in (4.2.26) is of larger order (in probability) than the first two terms. It follows that the probability

$$
\begin{aligned}
\beta_{L R, N}\left(p^{\prime}\right) & =P\left(\underline{t}^{*}>x_{k-1 ; \alpha_{N}}^{2}+\sigma(1) \mid p^{\prime}\right)= \\
& =P\left(\underline{t}^{*}>N{ }^{1 / 6} \tau(N)+\sigma(1) \mid p^{\prime}\right)
\end{aligned}
$$

converges uniformly to one for all $\mathrm{p}^{\prime} \in \Omega_{\mathrm{N}}^{(2)}$, in accordance with (4.2.24). 
Henceforth we assume that

$$
\zeta_{N} \stackrel{\text { def }}{=} \max _{i}\left|p_{i}^{\prime}-p_{i}^{0}\right| \leq N^{-1 / 5} \text {. }
$$

$\mathrm{Bij}(4.2 .13)$ and $(4.2 .19)$ the variance of the first term in the right-hand member of $(4.2 .26)$ is equal to

$$
\mathbb{N} \sum_{i} \frac{\left(p_{i}^{\prime}-p_{i}^{0}\right)^{2}}{p_{i}^{0}}\left(1+\sigma\left(\zeta_{N}\right)\right) \quad \text { for } N \rightarrow \infty \text {. }
$$

Suppose that $p^{\prime} \in \Omega_{N}^{(3)}$, i.e.

$$
\zeta_{\mathrm{N}} \leq(\varepsilon / \mathrm{k})^{\frac{1}{2}} \mathrm{~N}^{-5 / 12} \tau^{\frac{1}{2}}(\mathrm{~N})
$$

Then for sufficiently large $\mathbb{N}$ (independent of $\mathrm{p}^{\prime}$ ) the expression (4.2.28) is smaller than $\frac{3}{4} N^{1 / 6} \tau(N)$ and we deduce from (4.2.25) and lemma 4.2.1 that $\beta_{N}^{+}\left(p^{\prime}\right)$ and hence $\beta_{L R, N}\left(p^{\prime}\right)$ converges uniformly to zero for all $p^{\prime} \in \Omega_{N}^{(3)}$, in accordance with $(4.2 .24)$.

Next we consider the case where $p^{\prime} \in \Omega_{N}^{(4)}$. In this case the variance (4.2.28) tends uniformly to infinity for $\mathbb{N} \rightarrow \infty$, implying (cf.(4.2.26))

$$
P\left(\underline{t}^{*} \geq c_{N} \mid p^{\prime}\right)=P\left(\sum \underline{x}_{i} \log \left(p_{i}^{\prime} / p_{i}^{0}\right) \geq c_{N} \mid p^{\prime}\right)+\sigma(1)
$$

uniformly in $p^{\prime}$ for $N \rightarrow \infty$. From $(4.2 .14)$ and $(4.2 .19)$ we derive that

$$
\begin{gathered}
P\left(\underline{t}^{*} \geq c_{N} \mid p^{\prime}\right)= \\
=1-\Phi\left(\frac{\frac{1}{2} x_{k-1 ; \alpha_{N}^{2}}^{2}-\frac{1}{2} N\left[\frac{\left(p_{i}^{\prime}-p_{i}^{0}\right)^{2}}{p_{i}^{0}}+v(1)\right.}{N^{\frac{1}{2}}\left[\sum_{i} \frac{\left(p_{i}^{\prime}-p_{i}^{0}\right)^{2}}{p_{i}^{0}}\right]^{\frac{1}{2}}+v(1)}+v(1)\right.
\end{gathered}
$$

uniformly for $N \rightarrow \infty$ and $p^{\prime} \in \Omega_{N}^{(4)}$, which immediately yields (4.2.24).

It remains to consider $\Omega_{\mathrm{N}}^{(1)}$. Without loss of generality we assume that $\mathrm{p}_{1}^{\prime}<\varepsilon$. From $(4.2 .26)$ and $(4.2 .27)$, with $\mathrm{p}^{\prime}$ replaced by $\underline{x} / \mathbb{N}$ and $j=1$, we find that with probability one

$$
\underline{t}^{*} \geq \frac{1}{2}\left(\underline{x}_{1}-N p_{1}^{0}\right) \log \left(1+\frac{1}{2} \frac{\underline{x}_{1}-N p_{1}^{0}}{N_{1}^{0}}\right) \text {. }
$$


Hence it is easily verified that $P\left(\underline{t}^{*} \geq c \mid p^{\prime}\right)$ tends uniformly to one for $N \rightarrow \infty$ and $p^{\prime} \in \Omega_{N}^{(1)}$, which completes the proof of the lemma.

Q.E.D.

From the lemmata 4.2 .1 and 4.2 .3 we derive

Theorem 4.2 .1

Let $\alpha_{N}$ be a function of $N$ satisfying $\lim _{N \rightarrow \infty} \alpha_{N}=0$ and

(4.2.29) $\quad \log \alpha_{N}=\sigma\left(N^{1 / 6}\right)$ for $N \rightarrow \infty$.

Then

$$
\lim _{\mathbb{N} \rightarrow \infty} R_{L R, N}(p)=0 \quad \text { uniformly for all } p \in \Omega
$$

Proof:

To prove this theorem we need a more precise relation between $u_{\alpha_{N}}$ and $x_{\alpha_{N}}^{2}$ (we omit the subscript $k-1$ ) than (4.2.25). Since for $\mathbb{N} \rightarrow \infty$

$$
\alpha_{N}=1-\Phi\left(u_{\alpha_{N}}\right)=(2 \pi)^{-\frac{1}{2}} u_{\alpha_{N}}^{-1} \exp \left(-\frac{1}{2} u_{\alpha_{N}}^{2}\right) \cdot\left(1+O\left(u_{\alpha_{N}}^{-2}\right)\right)
$$

and

$$
\begin{aligned}
\alpha_{N}=P\left(x_{-k-1}^{2}>x_{\alpha_{N}}^{2}\right)= & \left\{2^{\frac{1}{2}(k-1)} \Gamma\left(\frac{k-1}{2}\right)\right\}^{-1}\left[x_{\alpha_{N}^{2}}\right]^{\frac{1}{2}(k-3)} . \\
& \cdot \exp \left(-\frac{1}{2} x_{\alpha_{N}^{2}}^{2}\right) \cdot\left(1+\mathcal{O}\left(\left[x_{\alpha_{N}}^{2}\right]^{-1}\right)\right)
\end{aligned}
$$

we find that for $\mathrm{N} \rightarrow \infty$

$$
x_{\alpha_{N}}^{2}-(k-3) \log x_{\alpha_{N}}^{2}+\Theta(1)=u_{\alpha_{N}}^{2}+2 \log u_{\alpha_{N}}
$$

and hence

$$
(4.2 .30) \quad x_{\alpha_{N}}^{2}=u_{\alpha_{N}}^{2}+\sigma\left(\log u_{\alpha_{N}}\right) \quad \text { for } N \rightarrow \infty
$$

Moreover, it is seen that if condition (4.2.29) holds, then the conditions of the lemmata 4.2 .1 and 4.2 .3 are also satisfied. We define

$(4.2 .31)$

$$
Q_{N}=N^{\frac{1}{2}}\left[\sum_{i=1}^{k} \frac{\left(p_{i}^{!}-p_{i}^{0}\right)^{2}}{p_{i}^{0}}\right]^{\frac{1}{2}}
$$


and divide the parameter space $\Omega$ for each $\mathbb{N}$ into three parts,

$$
\begin{aligned}
& \Omega_{N}^{(1)}=\left\{p^{\prime} \mid-\log u_{\alpha_{N}}<u_{\alpha_{N}}-Q_{N}<\log u_{\alpha_{N}}\right\}, \\
& \Omega_{N}^{(2)}=\left\{p^{\prime} \mid u_{\alpha_{N}}-Q_{N}<-\log u_{\alpha_{N}}\right\}, \\
& \Omega_{N}^{(3)}=\left\{p^{\prime} \mid u_{\alpha_{N}}-Q_{N}>\log u_{\alpha_{N}}\right\} .
\end{aligned}
$$

First we consider alternatives $p^{\prime} \in \Omega_{N}^{(1)}$. From $(4 \cdot 2 \cdot 10)$ and $(4 \cdot 2 \cdot 24)$ we obtain

$$
\begin{aligned}
R_{L R, N}\left(p^{\prime}\right) & =\beta_{N}^{+}\left(p^{\prime}\right)-\beta_{L R, N}\left(p^{\prime}\right)= \\
& =\Phi\left(\left(x_{\alpha_{N}}^{2}-Q_{N}^{2}\right) / 2 Q_{N}\right)-\Phi\left(u_{\alpha_{N}}-Q_{N}\right)+\sigma(1)
\end{aligned}
$$

for $\mathbb{N} \rightarrow \infty$, where $o(1)$ does not depend on $p^{\prime}$. We consider the difference of the arguments of both functions $\Phi$. By $(4.2 .30)$ we have

$$
\begin{aligned}
& \frac{1}{2 Q_{N}}\left(x_{\alpha_{N}}^{2}-Q_{N}^{2}\right)-\left(u_{\alpha_{N}}-Q_{N}\right)=\frac{1}{2 Q_{N}}\left[u_{\alpha_{N}}^{2}+Q_{N}^{2}-2 Q_{N} u_{\alpha_{N}}+O\left(\log u_{\alpha_{N}}\right)\right]= \\
= & \frac{1}{2 Q_{N}}\left[\left(u_{\alpha_{N}}-Q_{N}\right)^{2}+\sigma\left(\log u_{\alpha_{N}}\right)\right]<\frac{1}{2 Q_{N}} O\left(\log u_{\alpha_{N}}\right)= \\
= & \sigma\left(u_{\alpha_{N}}^{-1} \log u_{\alpha_{N}}\right)
\end{aligned}
$$

for $N \rightarrow \infty$ and hence the difference of both arguments tends uniformly to zero, implying that $R_{L R, N}$ tends uniformly to zero for $p^{\prime} \in \Omega_{\mathbb{N}}^{(1)}$.

Next we suppose that $p^{\prime} \in \Omega_{N}^{(2)}$. In this case we have

$$
\begin{aligned}
\beta_{L R, N}\left(p^{\prime}\right) & =1-\Phi\left(\left\{x_{\alpha_{N}}^{2}-Q_{N}^{2}\right\} / 2 Q_{N}\right)+\sigma(1) \\
& =1-\Phi\left(\left\{u_{\alpha_{N}}^{2}+\sigma\left(\log u_{\alpha_{N}}\right)-Q_{N}^{2}\right\} / 2 Q_{N}\right)+\sigma(1) \\
& >1-\Phi\left(\left\{Q_{N}^{2}-2 Q_{N} \log u_{\alpha_{N}}+\sigma\left(\log { }^{2} u_{\alpha_{N}}\right)-Q_{N}^{2}\right\} / 2 Q_{N}\right)+\sigma(1) \\
& =1-\Phi\left(-\log u_{\alpha_{N}}+\sigma\left(u_{\alpha_{N}}^{-1} \log ^{2} u_{\alpha_{N}}\right)\right)+\sigma(1)
\end{aligned}
$$

and hence $\beta_{L R, N}$ tends to one for $N \rightarrow \infty$ uniformly for all $p^{\prime} \in \Omega_{N}^{(2)}$, implying 
uniform convergence of $R_{L R, N}$ on this set also.

Finally, it is obvious that $\beta_{N}^{+}$and hence $R_{L R, N}$ converges uniformly to zero for $N \rightarrow \infty$ and all $\mathrm{p}^{\prime} \in \Omega_{\mathrm{N}}^{(3)}$. Q.E.D.
.

Although at first sight (4.2.29) seems to include very high rates of convergence of $\alpha_{N}$ to zero, it can easily be deduced from the preceding lemmata that under condition (4.2.29) the error of the second kind of the LR test (or of the MP test) for any fixed alternative tends much faster to zero than $\alpha_{N}$. Hence higher rates of convergence of $\alpha_{\mathbb{N}}$ to zero are also of interest. However, the work of RICHTER [41] shows that for higher rates of convergence of $\alpha_{N}$ the normal approximation to the multinomial distribution cannot be used to derive critical values of the LR test. Moreover, it is apparent from the work of LINNIK ([33] I,III) that the envelope power function cannot be obtained as before, since the critical value of the MP test statistic cannot be found by normal approximation as in lemma 4.2.1 if $\log \alpha_{N}$ is not of order $\sigma\left(N^{1 / 3}\right)$ for $N \rightarrow \infty$. Hence a different approach is necessary if faster rates of convergence of $\alpha_{N}$ are to be considered. 
[ 1 ] P. ALEXANDROFF, H. HOPF

Topologie 1, Springer, Berlin, 1935.

[2] N. BHATTACHARYA Sampling experiments on the combination of independent tests, Sankhya Ser.A $\underline{23}$ (1961), 191-196.

[3] P. BILLINGSLEY, F. TOPS $\varnothing \mathrm{E}$

Uniformity in weak convergence,

$\mathrm{Z}$. Wahrscheinlichkeitstheorie u. verw. Geb. 1 (1967), 1-16.

[ 4 ] A. BIRNBAUM

[5] A. BIRNBAUM

[6 ] Z.W. BIRNBAUM

[ 7 ] W.G. COCHRAN

[ 8 ] H. CRAMER

[9] F.N. DAVID

[10] F.N. DAVID

[11 ] R.B. DAVIES

[12 ] R.B. DAVIES
Combining independent tests of significance, J. Amer. Statist. Assoc. 49 (1954), 559-575.

Characterization of complete classes of tests of some multiparametric hypotheses, with applications to likelihood ratio tests, Ann. Math. Statist. 26 (1955) 21-36.

An inequality for Mill's ratio, Ann. Math, Statist. 13 (1942), 245-246.

Some methods for strengthening the common $\chi^{2}$ tests, Biometrics 10 (1954), 417-451.

Mathematical methods of statistics, Princeton Univ. Press, Princeton, 1946.

On the $\mathrm{P}_{\lambda_{\mathrm{n}}}$ test for randomness; remarks, further illustrations and table for $P_{\lambda_{n}}$, Biometrika $\underline{26}$ (1934), $1-11$.

Tables of ordinates and probability integral of the distribution of the correlation coefficient in small samples, Cambridge Univ. Press, Cambridge, First ed. 1938.

Concept of $\beta$-optimal, level- $\alpha$ tests of statistical hypotheses and an application to the summary evaluation of independent experiments, to be published.

The weighted combination of independent normal test statistics, to be published. 
[13] C. VAN EEDEN, J. HEMELRIJK

A test for equality of probabilities against a class of specified alternative hypotheses, including trend, I and II, Indag. Math. 17 (1955), 191-198 and 301-308.

[14] B. EFRON

[15] H.G. EGGLESTON

[16] PH. VAN ELTEREN

[17] R.A. FISHER

[18] I.J. GOOD

[19] I.J. GOOD

[20] H. HAHN, A. ROSENTHAL Increasing properties of Polya frequency functions, Ann. Math. Statist. 36 (1965), 272-279. Convexity, Cambridge Univ. Press, Cambridge, 1958. On the combination of independent two sample tests of Wilcoxon, Bull. Int. Statist. Inst. 37 III (1960), $351-360$.

Statistical methods for research workers, Oliver and Boyd, Edinburgh, London, $4^{\text {th }}$ ed. 1932. On the weighted combination of significance tests, J. Roy. Statist. Soc. Ser. B 17 (1955), 264-265. Significance tests in parallel and in series, J. Amer. Statist. Assoc. 53 (1958), 799-813.

Set functions, Univ. of New Mexico Press, Albuquerque, 1948.

[21] J. HEMELRIJK

Personal communication.

[22] D.G. HERR Asymptotically optimal tests for multivariate normal distributions, Ann. Math. Statist. 38 (1967), 1829-1844.

[23] W. HOEFFDING Asymptotically optimal tests for multinomial distributions, Ann. Math. Statist. 36 (1965), 369-401.

[24] W. HOEFFDING On probabilities of large deviations, Proc. $5^{\text {th }}$ Berkeley Symp. Math. Statist. Prob. I (1967), 203-220.

[25] S. KARLIN Total positivety, Stanford Univ. Press, Stanford,1968.

[26] W.M. KINCAID The combination of $2 \times \mathrm{m}$ contingency tables, Biometrics 18 (1962), 224-228.

[27] H.O. IANCASTER The combination of probabilities arising from data in discrete distributions, Biometrika 36(1949),370-382. 
[28] H.O. LANCASTER

The combination of probabilities: an application of orthonormal functions, Austr.J. Statist. $\underline{3}(1961), 20-33$.

[29] L. LE CAM An extension of Wald's theory of statistical decision functions, Ann. Math. Statist. 26 (1955), 69-81.

[30] E.L. LEHMANN On the existence of least favorable distributions, Ann. Math. Statist. 23 (1952), 408-416.

[31] E.L. LEHMANN Testing statistical hypotheses, Wiley, New York, London, 1959.

[32] E.L. LEHMANN, C.M. STEIN Most powerful tests of composite hypotheses. I Normal distributions, Ann. Math. Statist. 19 (1948), 495-516.

[33] Y.V. LINNIK Limit theorems for sums of independent variables taking into account large deviations, I and III, Theory Prob.Applications(English transl.) 4 ( 1961) 131-148 and $I$ (1962), 114-129.

[34] T. LIPTAK On the combination of independent tests, Magyar Tud. Akad.Mat. Kutató Int. Közl. $\underline{3}$ (1958), 171-197.

[35] T.K. MATTHES, D.R. TRUAX

Tests of composite hypotheses for the multivariate exponential family, Ann. Math. Statist. 38 (1967), 681-697.

[36] E.S. PEARSON

The probability integral transformation for testing goodness of fit and combining independent tests of significance, Biometrika 30 (1938), $134-148$.

[37] E.S. PEARSON On questions raised by the combination of tests based on discontinuous distributions, Biometrika $\underline{37}(1950), 383-398$. 
[38] K. PEARSON On a method of determining whether a sample of given size $n$ supposed to have been drawn from a parent population having a known probability integral has probably been drawn at random, Biometrika 25 (1933), 379-410.

[39] K. PEARSON On a new method of determining goodness of fit, Biometrika 26 (1934), 425-442.

[40] M.L. PURI The van Elteren $W$ test and non-null hypothesis, to be published.

[41] W. RICHTER Multidimensional limit theorems for large deviations and their application to the $x^{2}$ distribution, Theory Prob.Applications(English transl.) 2 (1964), 28-37.

[42] C.L. RUMKE, C. VAN EEDEN

Statistiek voor medici, Stafleu, Leiden, 1961.

[43] I.N. SANOV On the probability of large deviations of random variables, Select. Transl. Math. Statist. and Probability 1 (1961), 213-244.

[44] W. SCHAAFSMA, L.J. SMID

Most stringent somewhere most powerful tests against alternatives restricted by a number of linear inequalities, Ann. Math. Statist. $\underline{37}$ (1966), 1161-1172.

[45] W. SCHAAFSMA Hypothesis testing problems with the alternative restricted by a number of inequalities, thesis, Noordhoff, Groningen, 1966.

[46] W. SCHAAFSMA A comparison of the most stringent and the most stringent somewhere most powerful test for certain problems with restricted alternative, Ann. Math. Statist. 39 (1968), 531-546.

[47] R. SCHWARZ Admissible tests in multivariate analysis of variance, Ann. Math. Statist. 38 (1967), 698-710. 
[48] L.R. SHENTON

[49] C.M. STEIN

[50] P.V. SUKHATME

[51] L.H.C. TIPPETT

[52] E.C. TITCHMARSH

[53] A. WALD

[54] W.A. WALLIS

[55] B. WILKINSON

[56] F. YATES

[57] F. YATES

[58] M. ZELEN
Inequalities for the normal integral including a new continued fraction, Biometrika 41 (1954), $177-189$.

Admissibility of Hotelling's $T^{2}$ test, Ann. Math. Statist. 27 (1956), 616-623.

A contribution to the problem of two samples, Proc. Indian Acad. Sci. Ser. A 2 (1935),584-604. The method of statistics, Williams and Norgate, London, First ed. 1931.

The theory of functions, $2^{\text {nd }}$ ed., Oxford Univ. Press, Oxford, 1939.

Statistical decision functions, Wiley, New York, 1950 .

Compounding probabilities from independent significance tests, Econometrica 10 (1942), 229-248.

A statistical consideration in psychological research, Psych. Bull. 48 (1951), 156-158.

The use of transformations and maximum likelihood in the analysis of quantal experiments involving two treatments, Biometrika 42 (1955), 382-403.

A note on the application of the combination of probabilities test to a set of $2 \times 2$-tables, Biometrika 느 (1955), 404-411.

The analysis of incomplete block designs, J. Amer. Statist. Assoc. 52 (1957), 204-217.

[59] M. ZELEN, L.S. JOEL The weighted compounding of two independent significance tests, Ann. Math. Statist. 30 (1959), 885-895.

[60] W.R. VAN ZWET, J. OOSTERHOFF On the combination of independent test statistics, Ann. Math. Statist. 38 (1967), 659-680. 


\section{OTHER TITLES IN THE SERIES MATHEMATICAL CENTRE TRACTS}

A leaflet containing an order-form and abstracts of all publications mentioned below is available at the Mathematisch Centrum. Tweede Boerhaavestraat 49, Amsterdam-1005, The Netherlands. Orders should be sent to the same address.

MCT 1 T. VAN DER WALT, Fixed and almost fixed points, 1963. ISBN 906196 0029.

MCT 2 A.R. BLOEMENA, Sampling from a graph, 1964 . ISBN 9061960037.

MCT 3 G. DE LEVE, Generalized Markovian decision processes, part I: Model and method, 1964. ISBN 9061960045 .

MCT 4 G. DE LEVE, Generalized Markovian decision processes, part II: Probabilistic background, 1964. ISBN 9061960061

MCT 5 G. DE LEVE, H.C. TIJMS \& P.J. WEEDA, Generalized Markovian decision processes, Applications, 1970. ISBN 9061960517.

MCT 6 M.A. MAURICE, Compact ordered spaces, 1964. ISBN 9061960061.

MCT 7 W.R. VAN ZWET, Convex transformations of random variables, 1964. ISBN $906196007 \mathrm{x}$.

MCT 8 J.A. ZONNEVELD, Automatic numerical integration, 1964. ISBN 906196 0088 .

MCT 9 P.C. BAAYEN, Universal morphisms, 1964. ISBN 9061960096.

MCT 10 E.M. DE JAGER, Applications of distributions in mathematical physics, 1964. ISBN $906196010 \mathrm{x}$.

MCT 11 A.B. PAALMAN-DE MIRANDA, Topological semigroups, 1964. ISBN 906196 0118.

MCT 12 J.A.TH.M. VAN BERCKEL, H. BRANDT CORSTIUS, R.J. MOKKEN \& A. VAN WIJNGAARDEN, Formal properties of newspaper Dutch, 1965. ISBN 9061960134 .

MCT 13 H.A. LAUWERIER, Asymptotic expansions, 1966, out of print; replaced by MCT 54 .

MCT 14 H.A. IAUWERIER, Calculus of variations in mathematical physics, 1966. ISBN 9061960207 .

MCT 15 R. DOORNBOS, Slippage tests, 1966. ISBN 9061960215.

MCT 16 J.W. DE BAKKER, Formal definition of programming languages with an application to the definition of ALGOL 60, 1967. ISBN 906196 0223.

MCT 17 R.P. VAN DE RIET, Formula manipulation in ALGOL 60, part 1, 1968. ISBN 9061960258 .

MCT 18 R.P. VAN DE RIET, Formula manipulation in ALGOL 60, part 2, 1968. ISBN $906196038 \mathrm{X}$.

MCT 19 J. VAN DER SLOT, Some properties related to compactness, 1968. ISBN 9061960266 .

MCT 20 P.J. VAN DER HOUWEN, Finite difference methods for solving partial differential equations, 1968. ISBN 9061960274 . 
MCT 21 E. WATTEL, The compactness operator in set theory and topology, 1968. ISBN 9061960282.

MCT 22 T.J. DEKKER, ALGOL 60 procedures in numerical algebra, part 1, 1968. ISBN 9061960290 .

MCT 23 T.J. DEKKER \& W. HOFFMANN, ALGOL 60 procedures in numerical algebra, part 2, 1968. ISBN 9061960304.

MCT 24 J.W. DE BAKKER, Recursive procedures, 1971. ISBN 9061960606.

MCT 25 E.R. PAERL, Representations of the Lorentz group and projective geometry, 1969. ISBN 9061960398.

MCT 26 EUROPEAN MEETING 1968, Selected statistical papers, part I, 1968. ISBN 9061960312 .

MCT 27 EUROPEAN MEETING 1968, Selected statistical papers, part II, 1969. ISBN 9061960401.

MCT 28 J. DOSTERHOFF, Combination of one-sided statistical tests, 1969. ISBN $906196041 \mathrm{x}$.

MCT 29 J. VERHOEFF, Error detecting decimal codes, 1969. ISBN 9061960428.

MCT 30 H. BRANDT CORSTIUS, Excercises in computational linguistics, 1970. ISBN 9061960525 .

MCT 31 W. MOLENAAR, Approximations to the Poisson, binomial and hypergeometric distribution functions, 1970. ISBN 9061960533.

MCT 32 L. DE HAAN, On regular variation and its application to the weak convergence of sample extremes, 1970. ISBN 9061960541.

MCT 33 F.W. STEUTEL, Preservation of infinite divisibility under mixing and related topics, 1970. ISBN 9061960614.

MCT 34 I. JUHÁSZ, A. VERBEEK \& N.S. KROONENBERG, Cardinal functions in topology, 1971. ISBN 9061960622.

MCT 35 M.H. VAN EMDEN, An analysis of complexity, 1971. ISBN 9061960630.

MCT 36 J. GRASMAN, On the birth of boundary Zayers, 1971. ISBN 9061960649.

MCT 37 J.W. DE BAKKER, G.A. BLAAUW, A.J.W. DUIJVESTIJN, E.W. DIJKSTRA, P.J. VAN DER HOUWEN, G.A.M. KAMSTEEG-KEMPER, F.E.J. KRUSEMAN ARETZ, W.L. VAN DER POEL, J.P. SCHAAP-KRUSEMAN, M.V. WILKES \&. G. ZOUTENDIJK, MC-25 Informatica Symposium, 1971. ISBN 90 61960657.

MCT 38 W.A. VERLOREN VAN THEMAAT, Automatic analysis of Dutch compound words, 1971. ISBN 9061960738 .

MCT 39 H. BAVINCK, Jacobi series and approximation, 1972. ISBN 9061960746.

MCT 40 H.C. TIJMS, Analysis of $(s, S)$ inventory models, 1972. ISBN 9061960754.

MCT 41 A. VERBEEK, Superextensions of topological spaces, 1972. ISBN 90 61960762.

MCT 42 W. VERVAAT, Success epochs in Bernoulli trials (with applications in number theory), 1972. ISBN 9061960770.

MCT 43 F.H. RuYMGAART, Asymptotic theory of rank tests for independence, 1973. ISBN 9061960819.

MCT 44 H. BART, Meromorphic operator valued functions, 1973. ISBN 9061960827. 
MCT 45 A.A. BALKEMA, Monotone transformations and Zimit Zaws, 1973. ISBN 9061960835 .

MCT 46 R.P. VAN DE RIET, ABC ALGOI, A portable Zanguage for formula manipuZation systems, part 1: The Zanguage, 1973. ISBN 9061960843.

MCT 47 R.P. VAN DE RIET, ABC ALGOL A portable language for formula manipulation systems part 2: The compiler, 1973. ISBN 9061960851.

MCT 48 F.E.J. KRUSEMAN ARETZ, P.J.W. TEN HAGEN \& H.L. OUDSHOORN, In ALGOL 60 compiler in ALGOL 60, Text of the MC-compiler for the $E L-X 8,1973$. ISBN $906196086 \mathrm{x}$.

MCT 49 H. KoK, Connected orderable spaces, 1974. ISBN 9061960886.

MCT 50 A. VAN WIJNGAARDEN, B.J. MAILLOUX, J.E.L. PECK, C.H.A. KOSTER, M. SINTZOFF, C.H. LINDSEY, L.G.L.T. MEERTENS \& R.G. FISKER (eds.), Revised report on the algorithmic Zanguage ALGOL 68. ISBN 9061960894

MCT 51 A. HORDIJK, Dynamic programming and Markov potential theory, 1974. ISBN 9061960959.

MCT 52 P.C. BAAYEN (ed.), Topological structures, 1974. ISBN 9061960967.

MCT 53 M.J. FABER, Metrizability in generalized ordered spaces, 1974. ISBN 9061960975 .

MCT 54 H.A. LAUWERIER, Asymptotic analysis, part 1, 1974. ISBN 9061960983.

MCT 55 M. HALL JR. \& J.H. VAN LINT (eds.), Combinatorics, part 1: Theory of designs finite geometry and coding theory, 1974. ISBN 9061960991 .

MCT 56 M. HALL JR. \& J.H. VAN LINT (eds.), Combinatorics, part 2: Graph theory; foundations, partitions and combinatorial geometry, 1974. ISBN 9061961009.

MCT 57 M. HALL JR. \& J.H. VAN LINT (eds.), Combinatorics, part 3: Combinatorial group theory, 1974. ISBN 9061961017.

MCT 58 W. ALBERS, Asymptotic expansions and the deficiency concept in statistics, 1975. ISBN 9061961025 .

MCT 59 J.L. MIJNHEER, Sample path properties of stable processes, 1975. ISBN 9061961076 .

MCT 60 F. GÖBEL, Queueing models involving buffers. ISBN 9061961084.

* MCT 61 P. VAN EMDE BOAS, Abstract resource-bound classes, part 1. ISBN 9061961092 .

* MCT 62 P. VAN EMDE BOAS, Abstract resource-bound classes, part 2. ISBN 9061961106 .

MCT 63 J.W. DE BAKKER (ed.), Foundations of computer science, 1975. ISBN 9061961114

MCT 64 W.J. DE SCHIPPER, Symmetrics closed categories, 1975. ISBN 906196 1122 .

MCT 65 J. DE VRIES, Topological transformation groups 1 A categorical approach, 1975. ISBN 9061961130 .

* MCT 66 H.G.J. PIJLS, Locally convex algebras in spectral theory and eigenfunction expansions. ISBN 9061961149. 
* MCT 67 H.A. LAUWERIER, Asymptotic analysis, part 2. ISBN $906196119 \mathrm{x}$.

* MCT 68 P.P.N. DE GROEN, Singulary pertlibed differential operators of second order. ISBN 9061961203.

* MCT 69 J.K. LENSTRA, Sequencing by enumerative methods. ISBN 9061961254 .

MCT 70 W.P. DE ROEVER JR., Recursive program schemes: semantics and proof theory. ISBN 9061961270 .

* MCT 71 J.A.E.E. VAN NUNEN, Contracting Markov decision processes. ISBN 9061961297.

* MCT 72 J.K.M. JANSEN, Simple periodic and nonperiodic lamé functions and their applications in the theory of conical waveguides. ISBN 9061961300 .

* MCT 73 D.M.R. LeIVANT, Absoluteness of intuitionistic logic. ISBN $906196122 \mathrm{x}$.

* MCT 74 H.J.J. TE RIELE, A theoretical and computational study of generalized aliquot sequences. ISBN 9061961319.

An asterisk before the number means "to appear". 